\title{
Logarithmic Local Energy Decay for Scalar Waves on a General Class of Asymptotically Flat Spacetimes
}

\author{
Georgios Moschidis ${ }^{1}$
}

Received: 16 December 2015 / Accepted: 25 March 2016 / Published online: 27 April 2016

(C) Springer International Publishing AG 2016

\begin{abstract}
This paper establishes that on the domain of outer communications of a general class of stationary and asymptotically flat Lorentzian manifolds of dimension $d+1, d \geq 3$, the local energy of solutions to the scalar wave equation $\square_{g} \psi=0$ decays at least with an inverse logarithmic rate. This class of Lorentzian manifolds includes (non-extremal) black hole spacetimes with no restriction on the nature of the trapped set. Spacetimes in this class are moreover allowed to have a small ergoregion, but are required to satisfy an energy boundedness statement. Without making further assumptions, this logarithmic decay rate is shown to be sharp. Our results can be viewed as a generalisation of a result of Burq, dealing with the case of the wave equation on flat space outside compact obstacles, and results of Rodnianski-Tao for asymptotically conic product Lorentzian manifolds. The proof will bridge ideas from Rodnianski and Tao (see [58]) with techniques developed in the black hole setting by Dafermos and Rodnianski (see [21,22]). As a soft corollary of our results, we will infer an asymptotic completeness statement for the wave equation on the spacetimes considered in the case where no ergoregion is present.
\end{abstract}

Keywords Asymptotically flat · Black holes · Logarithmic decay · Wave equation

\section{Contents}

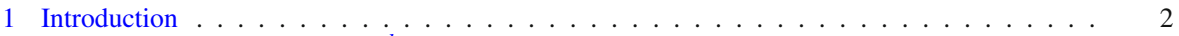

1.1 The Wave Equation on $\mathbb{R}^{d}$ with Obstacles and a Result of Burq . . . . . . . . . . . . . . . . . . . . . . . .

1.2 A Result of Rodnianski-Tao for Product Lorentzian Manifolds . . . . . . . . . . . . . . . 6

$\bowtie$ Georgios Moschidis gm6@math.princeton.edu

1 Department of Mathematics, Princeton University, Fine Hall, Washington Road, Princeton, NJ 08544, USA 
1.3 The Main Result: Logarithmic Decay in a Suitably General Asymptotically Flat Setting . 7

1.4 Necessity of Assumptions $1-4 \ldots \ldots \ldots \ldots$. . . . . . . . . . . . . . . . . . . . . . . . . . . .

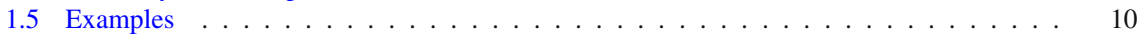

2 Statement of the Theorem and Sketch of the Proof . . . . . . . . . . . . . . . . . . 11

2.1 Geometric Assumptions on the Background Spacetime . . . . . . . . . . . . . . . . . 11

2.2 Examples of Spacetimes Satisfying Assumptions 1-4 . . . . . . . . . . . . . . . . . . . 17

2.3 Statement of the Decay Result . . . . . . . . . . . . . . . . . . . . . . . . . . . . . . . . . . . . . . . . .

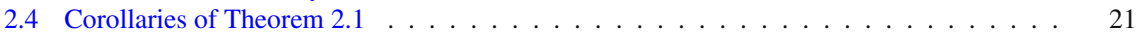

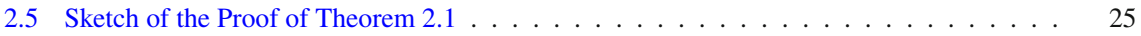

2.6 Remark on the Proof of Theorem $2.1 \ldots \ldots \ldots$. . . . . . . . . . . . . . . . . . . . . . . . . . . . . . . . . . . . . . . . . . .

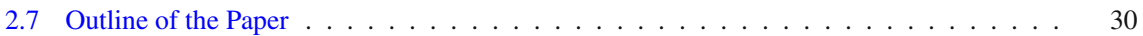

3 Notational Conventions and Hardy Inequalities . . . . . . . . . . . . . . . . . . . . . 31

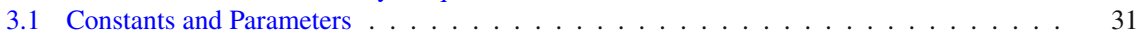

3.2 Inequality Symbols . . . . . . . . . . . . . . . . . . . . . . . 31

3.3 Coordinate Charts on $\mathcal{D}$ and Subsets of $\mathcal{D}$ Associated to $t$. . . . . . . . . . . . . . . 32

3.4 Connections and Volume Forms . . . . . . . . . . . . . . . . . . . . . . . . . . . . . . . . . . . .

3.5 Integration over Domains and Hypersurfaces . . . . . . . . . . . . . . . . . . . . . . . . . . . . . . . . . . . . .

3.6 Notations for Derivatives on $\mathbb{s}^{d-1} \ldots \ldots \ldots \ldots \ldots$

3.7 The $O_{k}(\cdot)$ Notation . . . . . . . . . . . . . . . . . . . . . . . . . . . 35

3.8 Vector Field Multipliers and Currents . . . . . . . . . . . . . . . . . . . . . . 36

3.9 Hardy-Type Inequalities . . . . . . . . . . . . . . . . . . . . . . . . . . . . . . . . . . . . . . .

4 Construction of the Frequency Decomposed Components of $\psi$. . . . . . . . . . . . . . . . . 37

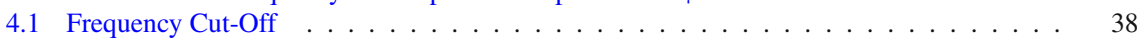

4.2 Bounds for the Frequency-Decomposed Components . . . . . . . . . . . . . . . . . . . 40

5 Estimates for $\psi_{k}$ in the Asymptotically Flat Region . . . . . . . . . . . . . . . . . . . . . . . . . 60

5.1 Some $\partial_{r}$-Morawetz Type Estimates . . . . . . . . . . . . . . . . . . . . . . . . . . . 61

5.2 Some $r^{p}$-Weighted Energy Estimates . . . . . . . . . . . . . . . . . . . . . . . . . . . . . . . . . . . . . . . . . . .

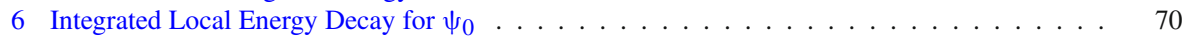

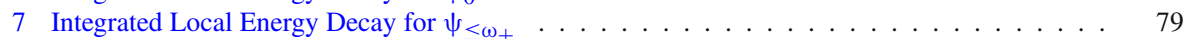

7.1 A Carleman-Type Estimate for $\bar{\psi}_{k}, 1 \leq|k| \leq n \ldots \ldots \ldots$. . . . . . . . . . 80

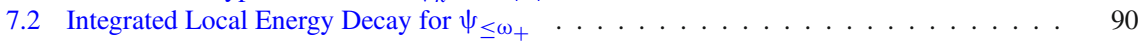

8 Polynomial Decay of the Local Energy of $\psi_{\leq \omega_{+}} \ldots \ldots$. . . . . . . . . . . . . . . . . . . . . . 100

9 The Frequency Interpolation Argument . . . . . . . . . . . . . . . . . . . . . . . . . . 102

10 Proof of Corollary $2.2 \ldots \ldots \ldots \ldots \ldots$

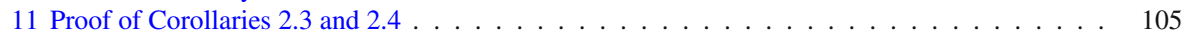

11.1 Proof of Corollary $2.3 \ldots \ldots \ldots \ldots \ldots$

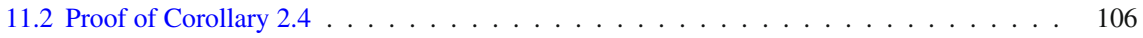

12 Sharpness of the Logarithmic Decay Statement . . . . . . . . . . . . . . . . . . . . . . . . . . . . . . . . . .

A Construction of the $(u, r, \sigma)$ Coordinate Chart . . . . . . . . . . . . . . . . . . . 117

B Proof of the Inclusion $J^{-}(p) \cap \Sigma \subseteq \Sigma \cap \mathcal{D}$ for $p \in J^{+}(\Sigma) \cap \mathcal{D} \ldots \ldots \ldots \ldots$. . . . . . . . 118

C Interpolation for $r$-Weighted Energy Bounds . . . . . . . . . . . . . . . . . . . . . . . 119

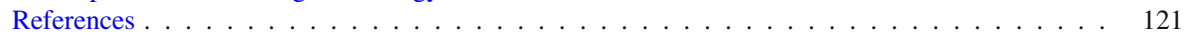

\section{Introduction}

Recent progress in understanding the behaviour of solutions to the scalar wave equation

$$
\square_{g} \psi=0
$$

on various general relativistic backgrounds has been astonishing: In the case of the Schwarzschild exterior background, boundedness and decay results were established in $[6,7,14,15,18,43]$. In the case of the Kerr family, similar results in the very slowly rotating case (i.e. for angular momentum $a$ and mass $M$ satisfying $|a| \ll M$ ) were obtained in $[1,19,21,22,66]$. The full subextremal range $|a|<M$ was finally treated 
in $[17,23,64]$. See also [13] for the Kerr-Newman case. Various refinements of the earlier decay results on these spacetimes appear in $[45,46,62]$. This picture changes dramatically when one switches attention to extremal black hole spacetimes, where instability results have been established in [2-5].

All the preceding examples dealt with asymptotically flat spacetimes, but a plethora of relevant results have also been proven for spacetimes with different asymptotic structure: See [16,30,31,48,67] for the case of Schwarzschild- and Kerr-deSitter spacetimes, and [38-40] for the case of Kerr-AdS spacetimes.

Given the amount of technical machinery that has been developed by the aforementioned authors, it is now feasible to move out of the realm of backgrounds that are algebraically special solutions to the Einstein equations or perturbations thereof and address questions regarding the behaviour of scalar waves on more general Lorentzian manifolds $\left(\mathcal{M}^{d+1}, g\right)$. A demanding first question in this direction is the following:

What are the most general types of spacetimes $\left(\mathcal{M}^{d+1}, g\right)$ on which boundedness and decay of solutions to the scalar wave equation (1.1) can be obtained and studied? Or, from a different perspective, what are the possible obstructions to stability for (1.1) on general backgrounds?

This paper aims to make a step towards providing answers to the above question, by establishing the following general decay result:

Theorem Let $\left(\mathcal{M}^{d+1}, g\right), d \geq 3$, be a globally hyperbolic spacetime, which is stationary and asymptotically flat, and which can possibly contain black holes with a non degenerate horizon and a small ergoregion. Moreover, suppose that an energy boundedness statement is true for solutions $\psi$ to (1.1) on the domain of outer communications $\mathcal{D}$ of $(\mathcal{M}, g)$. Then the local energy of $\psi$ on $\mathcal{D}$ decays at least with a logarithmic rate:

$$
E_{l o c}(t) \lesssim_{m} \frac{1}{\{\log (2+t)\}^{2 m}} E_{w}^{(m)}(0),
$$

where $t$ is a suitable time function on $\mathcal{D}$ and $E_{w}^{(m)}(0)$ is a weighted initial energy of the first $m$ derivatives of $\psi$.

A more detailed and rigorous statement of this theorem and the assumptions on the spacetimes under consideration will be presented in Sections 2.1 and 2.3.

As an application of our results, we will deduce quantitative decay rates for solutions to (1.1) on a number of vacuum (and other) spacetime backgrounds which appear in the literature, but whose trapping structure is not yet completely understood. These examples include, for instance, axisymmetric scalar waves on the Emparan-Reall black rings (see [34]) and the Elvang-Figueras black Saturn (see [32]). Furthermore, the results of the current paper will be used to rigorously establish the so called Friedman ergosphere instability (introduced and supported heuristically in [35]). That is to say, we will establish by contradiction that no non-degenerate energy boundedness statement can hold on stationary spacetimes with ergoregion and no event horizon; see our forthcoming [51].

As a corollary of the above theorem, using also the results of [52], we will infer that the energy flux of solutions $\psi$ to (1.1) through a foliation of hyperboloidal hypersurfaces decays logarithmically in time. This result will in turn yield an asymp- 
totic completeness statement for the wave equation on spacetimes $(\mathcal{M}, g)$ satisfying the assumptions of the above theorem but without ergoregion. See Sections 2, 10 and 11 .

In the context of non-asymptotically flat spacetimes, we should note the results of Vasy [67], dealing with a class of Lorentzian manifolds generalising Kerr-de Sitter spacetime. For [67], however, the structure of the trapped null geodesics of the underlying manifold and the deSitter asymptotics play a crucial role in establishing exponential decay rates for solutions to the wave equation $\square_{g} \psi=0$. In particular, the trapped set is required to resemble closely that of Kerr-de Sitter spacetime. In contrast, in our setting no structural condition is placed on the trapped set, which leads to an inverse logarithmic decay rate (1.2) for the local energy of scalar waves which is sharp for some of the spacetimes in the class under consideration. Notice also that the asymptotic flatness of the spacetimes considered here would prohibit establishing faster than polynomial decay rates for solutions to (1.1).

Before stating more precisely the main result of the current paper, we will first examine two well understood examples: Solutions of (1.1) on flat space outside a compact obstacle and solutions to (1.1) on asymptotically conic manifolds of product type. It is the results of Burq [8], in the obstacle case, and of Rodnianski-Tao [58], in the product case, that our main theorem generalises.

\subsection{The Wave Equation on $\mathbb{R}^{d}$ with Obstacles and a Result of Burq}

Let $\mathcal{O}$ be a compact subset of $\mathbb{R}^{d}$, which is the closure of a finite number of domains with smooth boundary. Let also $\psi=\psi(t, x) \in C^{\infty}\left(\mathbb{R} \times\left(\mathbb{R}^{n} \backslash \mathcal{O}\right)\right)$ solve

$$
\left(\partial_{t}^{2}-\Delta\right) \psi=0
$$

on $\mathbb{R} \times\left(\mathbb{R}^{d} \backslash \mathcal{O}\right)$, with $\psi \equiv 0$ on $\partial \mathcal{O}$ and with $\left(\psi, \partial_{t} \psi\right)$ compactly supported (or at least suitably decaying) on $\{t=0\}$. In this case, one immediately sees that there exists a positive-definite conserved energy

$$
E(t)=\int_{\mathbb{R}^{d} \backslash \mathcal{O}}|\partial \psi(t, x)|^{2} d x
$$

(corresponding to the Killing field $\partial_{t}$ on $\mathbb{R}^{d+1} \backslash(\mathbb{R} \times \mathcal{O})$ ), which allows to easily handle boundedness issues.

Starting from the boundedness of the energy $E(t)$, "soft" arguments can be used to infer that for any $R>0$, the local energy $E_{R}(t)$ (i.e. the energy contained in a ball $B_{R}$ of $\mathbb{R}^{d}$ of fixed radius $R$ ) tends to 0 as $t \rightarrow+\infty$. Attempting to study the precise rate at which the local energy decays, it is inevitable that the nature of trapping ${ }^{1}$ will

\footnotetext{
${ }^{1}$ Line rays that are "reflected" on the obstacle's surface and remain in a bounded region of space for arbitrarily long time are said to be trapped. An obstacle is called non-trapping if it does not give rise to a trapped ray.
} 
come into play. This subject has been extensively studied during the last 50 years, but here we will refer to only a few indicative results. In the case the obstacle $\mathcal{O} \subseteq \mathbb{R}^{d}$ is non-trapping, Morawetz, Ralston and Strauss (see [49]) showed that for any function $\psi$ solving $\left(\partial_{t}^{2}-\Delta\right) \psi=0$ on $\mathbb{R} \times\left(\mathbb{R}^{d} \backslash \mathcal{O}\right)$, with $\psi=0$ on $\partial \mathcal{O}$ and $\left.\left(\psi, \partial_{t} \psi\right)\right|_{t=0}$ compactly supported, the local energy

$$
E_{R}(t) \doteq \int_{B_{R} \backslash \mathcal{O}}|\partial \psi(t, x)|^{2} d x
$$

decays at least polynomially in time $t$, and in fact this decay rate becomes exponential if the space dimension $d$ is odd. In order to establish this decay rate, it was first shown that

$$
\int_{0}^{\infty} E_{R}(t) d t \leq C(R) \cdot E(0)
$$

where $E(0)=\int_{\mathbb{R}^{d} \backslash \mathcal{O}}|\partial \psi(0, x)|^{2} d x$ is the initial energy of the wave. An inequality of the form (1.5) is usually referred to as an integrated local energy decay estimate.

Due to a result by Ralston (see [55]), however, the presence of even a single trapped ray is inconsistent with any quantitative decay rate for the local energy of waves in terms only of their initial energy. Moreover, no statement of the form (1.5) can be valid. This fact has been recently generalised to the case of globally hyperbolic Lorentzian manifolds with trapped null geodesics by Sbierski in [59].

If one is willing to "sacrifice" some initial regularity on the right hand side of (1.5), so as to establish a quantitative decay estimate for the local energy of a wave $\psi$ in terms of the initial energy of higher order derivatives of $\psi$, then one can still obtain various types of decay rates, which are expected to become faster as the trapping becomes more unstable (compare for instance [41] and [56]). It is remarkable, therefore, that in [8] Burq was able to prove without any assumptions regarding the nature of trapping and the form of the obstacle $\mathcal{O}$, and with the hypotheses on $\psi$ as before, that one has

$$
E_{R}(t) \lesssim\{\log (2+t)\}^{-2 m} \cdot E^{(m)}(0),
$$

where $E^{(m)}(0)$ denotes the energy of the first $m$ derivatives of $\psi$ at $\{t=0\}$. In the preceding $\lesssim$ notation, the implicit constant depends on $R$ and on the size of the compact support of the initial data for $\psi$ on $\{t=0\}$.

In fact, Burq's result is a bit more general, as it allowed for $\psi$ satisfying an equation of the form

$$
\partial_{t}^{2} \psi-\partial_{i}\left(a^{i j} \partial_{j}\right) \psi=0
$$

where $a^{i j}=a^{i j}(x)$ is a smooth positive definite matrix which is equal to the identity outside some large compact set. However, the fact that this operator is identical to $\partial_{t}^{2}-\Delta$ outside a large spatial ball was used in an essential way in Burq's argument, which relied on a decomposition of $\psi$ into spherical harmonics outside a large ball and on using explicit representation formulas to study the asymptotics of the resulting Hankel functions. Hence, his proof does not immediately generalise to the case where $\left\{a^{i j}\right\}-I d$ is not compactly supported. 


\subsection{A Result of Rodnianski-Tao for Product Lorentzian Manifolds}

The next step after studying the wave equation on $\mathbb{R}^{d}$ outside a compact obstacle is addressing the wave equation on product Lorentzian manifolds with a more general asymptotically flat structure.

Given an arbitrary Riemannian manifold $\left(\mathcal{N}^{d}, \bar{g}\right)$, quantitative estimates for the resolvent $R\left(\lambda+i \varepsilon ; \Delta_{\bar{g}}\right)$ of the Laplacian $\Delta_{\bar{g}}$ on $(\mathcal{N}, g)$ are closely connected to estimates for solutions to the wave equation $\square_{g} \psi=0$ on the product Lorentzian manifold $(\mathbb{R} \times \mathcal{N}, g)$ with $g=-d t^{2}+\bar{g}, t$ being the projection to the first factor of $\mathbb{R} \times \mathcal{N}$ (see e.g. [58,60,61] and references therein). ${ }^{2}$ Therefore, relevant to our question on the behaviour of scalar waves on general Lorentzian manifolds are the results of Rodnianski and Tao [58]. In particular, in [58] the authors have established (among other results) quantitative bounds for such resolvents for a general class of asymptotically conic Riemannian manifolds $\mathcal{N}$ of dimension $d \geq 3$. This enabled them to obtain, as a corollary of their resolvent estimates, a decay without a rate result for solutions to the wave equation on $(\mathbb{R} \times \mathcal{N}, g)$.

Though not explicitly stated, the results established in [58] are also sufficient to obtain a logarithmic decay statement for scalar waves on $(\mathbb{R} \times \mathcal{N}, g)$ : The exponential bounds on the resolvent $R\left(\lambda+i \varepsilon ; \Delta_{\bar{g}}\right)$ as $\lambda \rightarrow \infty$, as well as the bounds for the resolvent near $\lambda=0$, suffice to establish that on the product manifold $(\mathbb{R} \times \mathcal{N}, g)$, the local energy of solutions $\psi$ to the wave equation decays with a rate of the form:

$$
E_{R}(\tau) \lesssim\{\log (2+\tau)\}^{-2 m} \cdot E_{w}^{(m)}(0)
$$

In the above, $E_{R}(t)=\int_{\{t=\tau\} \cap\{r \leq R\}}\left(\left|\partial_{t} \psi\right|^{2}+\left|\nabla^{(\mathcal{N})} \psi\right|^{2}\right)$ is the local energy contained in the region $\{r \leq R\}$ at time $\tau$, for some function $r \geq 0$ tending to $+\infty$ towards the asymptotically conic end of $\mathcal{N}$, and $E_{w}^{(m)}(0)$ is a suitable weighted energy of the first $m$ derivatives of $\psi$ at $\{t=0\}$. Moreover, the implicit constant in the preceding $\lesssim$ notation depends only on the geometry of $(\mathcal{N}, \bar{g})$, and not on the size of the support of the intial data for $\psi$.

Thus, the results of [58] generalise the results of Burq in [8] to the case of product asymptotically conic Lorentzian manifods, that are not necessarily identical to $\left(\mathbb{R}^{d+1}, \eta\right)$ outside a bounded cylinder, $\eta$ being the usual Minkowski metric on $\mathbb{R}^{d+1}$. See also the work of Cardoso and Vodev $[9,10]$, establishing resolvent estimates similar to the ones in [58], but restricted to the high frequency regime $\lambda \gg 1$ (and obtained by different methods), extending the logarithmic decay results of Burq to a general class of product Lorentzian manifods. For an alternative derivation of similar high frequency resolvent estimates for Schrödinger operators with weak decay and regularity assumptions on the potential, see [25].

We will now proceed to state the main result of this paper, which should be viewed as a generalisation of the results of [58] to not necessarily product Lorentzian manifolds, which are moreover allowed to contain black hole regions and a small ergoregion. We

\footnotetext{
2 Notice that on such a Lorentzian manifold, the Killing field $\partial_{t}$ immediately gives rise to a bounded (in fact preserved) energy $\int_{t=\text { const }} J_{\mu}^{\partial_{t}}(\psi) n^{\mu}$ for solutions $\psi$ to the wave equation $\square_{g} \psi=0$.
} 
remark already that many of the ideas of the proof of the main result of the current paper actually originate in [58]. The importance of the ideas and techniques of [58] in our setting will become evident in the subsequent sections.

\subsection{The Main Result: Logarithmic Decay in a Suitably General Asymptotically Flat Setting}

The aforementioned results of Burq and Rodnianski-Tao indicate that if one wants to study a more general class of asymptotically flat spacetimes, possibly containing a black hole and a small ergoregion, and with few further restrictions on the geometry of the region between the horizon (if non empty) and "infinity", one can only hope to prove a logarithmic decay rate for the local energy of solutions to (1.1). This is exactly the result that we will prove here.

In particular, we will be concerned with globally hyperbolic spacetimes $\left(\mathcal{M}^{d+1}, g\right)$, $d \geq 3$, satisfying the following assumptions:

1. Asymptotic flatness and stationarity: The metric $\bar{g}$ and the second fundamental form $k$ induced on a Cauchy hypersurface $\Sigma$ of $(\mathcal{M}, g)$ must form an asymptotically flat triad $(\Sigma, \bar{g}, k)$. Moreover, there must exist a Killing field $T$ (called the stationary Killing field) with complete orbits on the domain of outer communications $\mathcal{D}$ of $(\mathcal{M}, g)$, extending also to the boundary of $\mathcal{D}$, such that $T$ becomes strictly timelike in the asymptotically flat region of $(\mathcal{M}, g)$. See Section 2.1 for a precise definition of these notions.

2. Killing horizon with positive surface gravity: If $(\mathcal{M}, g)$ is not identical to the domain of outer communications $\mathcal{D}$, then the boundary $\mathcal{H}$ of $\mathcal{D}$ (called the event horizon of the spacetime) must be a non degenerate Killing horizon (possibly for a different Killing vector field than $T$ ), with positive surface gravity.

3. Smallness of the ergoregion: The subset $\{g(T, T) \geq 0\}$ of the domain of outer communications $\mathcal{D}$ must be quantitatively small, as should be the maximum positive value of $g(T, T)$. If $\mathcal{H}=\emptyset$, then this requirement reduces to the assumption that the ergoregion being empty. ${ }^{3}$

4. Boundedness of the energy of waves: The energy of solutions $\psi$ to the scalar wave equation $\square_{g} \psi=0$ with respect to a $T$-invariant, globally timelike vector field $N$ and a suitable foliation of the domain of outer communications $\mathcal{D}$ must remain bounded by a constant times the energy of $\psi$ initially.

These assumptions will be stated more precisely in Section 2.1. The main result of this paper can be stated as follows:

Theorem 1.1 Let $\left(\mathcal{M}^{d+1}, g\right), d \geq 3$, be a spacetime satisfying the hypotheses $1-4$, and let $\mathcal{D}$ be its domain of outer communications. Then any smooth solution $\psi$ to $\square_{g} \psi=0$ on $\mathcal{D}$ with suitably decaying initial data on a Cauchy hypersurface $\Sigma$ of $\mathcal{M}$ satisfies for any integer $m>0$ and any $0<\delta_{0} \leq 1$ :

\footnotetext{
3 For the energy instability associated to the existence of an ergoregion in the absence of an event horizon, see already our forthcoming [51]. We will return to this issue at the discussion in the next section.
} 


$$
E_{l o c}(t) \leq \frac{C_{m}}{\{\log (2+t)\}^{2 m}} E^{(m)}(0)+\frac{C}{t^{\delta 0}} E_{w, \delta_{0}}(0) .
$$

In the above, $t \geq 0$ is a suitable time function on $J^{+}(\Sigma) \cap \mathcal{D}\left(J^{+}(\Sigma)\right.$ being the causal future of $\Sigma$ ) with $\{t=0\} \equiv \Sigma \cap \mathcal{D}$, and $E_{\text {loc }}(t)$ is the local energy of the wave at time $t . E^{(m)}(0)$ is the energy of the first $m$ derivatives of $\psi$ at $\{t=0\}$, while $E_{w, \delta_{0}}(0)$ is a suitable weighted energy of $\psi$ at $\{t=0\}$. The constant $C$ on the right hand side depends on the size of the ball where the local energy $E_{l o c}$ is measured and on the geometry of $(\mathcal{D}, g)$, while in addition to that, $C_{m}$ also depends on the number $m$ of derivatives of $\psi$ in $E^{(m)}(0)$.

A more precise statement of the above result will be given in Section 2.3, after a more detailed discussion of the geometric hypotheses on $(\mathcal{M}, g)$.

This result can be immediately applied to many interesting black hole spacetimes, on many of which no decay result had been established so far. These examples will be discussed in Section 1.5.

Let us also mention here that our result is a proper generalisation of the theorem of Burq (at least in dimensions $d \geq 3$ ): While not explicitly mentioned later in this paper, the proof of Theorem 1.1 can also be repeated in the case of the wave equation on $\mathbb{R}^{d}$ with obstacles, relaxing the assumption of $a^{i j}$ in the perturbed Laplacian $\partial_{i}\left(a^{i j} \partial_{j}\right)$ being equal to $I d$ outside a compact set (as required by Burq) to just demanding that $\left(a^{i j}\right) \rightarrow I d$ as $r \rightarrow \infty$ in a quantitative manner. Moreover, the constants in the final inequality do not depend on the size of the support of the initial data for $\psi$ (which in particular needs not be compactly supported initially); only the finiteness of a suitable weighted norm of an initial higher order energy of $\psi$ is necessary.

\subsection{Necessity of Assumptions 1-4}

At this point, a few words should be said regarding the necessity of Assumptions 1-4 on the spacetimes under consideration. We will see that while each of these assumptions can possibly be relaxed, discarding completely any of them (without adding any extra hypothesis in its place) appears to lead to spacetimes where Theorem 1.1 does not hold.

Some assumption of global character, such as Assumption 1 on stationarity and asymptotic flatness, seems to be necessary for a first statement of the generality we are after. In particular, Assumption 1 seems to be the most appropriate for applications in cases of isolated self gravitating relativistic systems satisfying the Einstein equations (with suitable matter fields) with zero cosmological constant.

Replacing the asymptotic flatness assumption with other asymptotics can lead to instability results contradicting Theorem 1.1: This is the case for asymptotically AdS spacetimes, for instance (with suitable boundary conditions at the timelike conformal boundary considered as in [38]). In fact, on Anti-deSitter space itself, while the usual $\partial_{t}$ Killing vector field gives rise to a conserved non degenerate energy current, there exist time periodic solutions to the wave equation, and this fact prohibits the proof of any sort of uniform decay rate (see [40] and references therein). 
Remark The presence of a non-empty non-degenerate horizon $\mathcal{H}^{+}$in an asymptotically AdS spacetime might be sufficient to provide stability results for scalar waves, at least in the case where a boundedness statement for the energy of solutions to $\square \psi=0$ is true, as the results on Kerr-AdS suggest: In [39], Holzegel and Smulevici show that under certain bounds on the value of the cosmological constant $\Lambda$, the angular momentum $a$, the mass $M$ of the Kerr-AdS spacetime and the mass $m$, implying among other things the existence of a globally causal Killing field, any solution $\psi$ to the massive wave equation

$$
\square_{g} \psi+m \psi=0
$$

decays logarithmically in time (provided, of course, that some weighted energy of the initial data is finite). And in fact, this decay rate is optimal (see [40], and also [37]). Hence, it would be of particular interest to explore the minimal geometric conditions that need to be imposed on a general asymptotically AdS spacetime with a non-degenerate event horizon, in order for a logarithmic decay estimate for solutions to (1.9) to hold. Notice, of course, that such a decay estimate can not hold on all asymptotically AdS spacetimes containing a non-degenerate horizon, since there exist examples of such spacetimes on which (1.9) is actually unstable: In the recent [29], Dold constructed exponentially growing solutions to the massive wave equation on Kerr-AdS spacetimes violating the Hawking-Reall bound.

Concerning Assumption 2 on the non-degeneracy of the horizon $\mathcal{H}^{+}$, we notice that the instability results of Aretakis [2-5] on extremal black holes are rooted in the absence of a red shift type vector field near $\mathcal{H}^{+}$in the sense of [18]. This fact suggests that dispensing with Assumption 2 might lead to spacetimes on which Theorem 1.1 is not true. Notice, however, that Aretakis in [2,3] was able to exploit additional geometric features of the horizon of extremal Reissner-Nordström spacetime in order to obtain stability results for the first order energy of a scalar wave $\psi$. Thus, it may be possible to replace Assumption 2 by some weaker assumption regarding the geometry of $\mathcal{H}$, that allows interesting extremal examples. ${ }^{4}$

Assumption 3 on the smallness of the ergoregion, if violated, will contradict any decay statement for the local energy of scalar waves: On a spacetime with a spatially compact ergoregion and no horizon, for instance, a solution $\psi$ to $\square \psi=0$ with initial $T$ energy $\int_{t=0} J_{\mu}^{T}(\psi) n^{\mu}=-1$ will satisfy $\int_{t=\tau} J_{\mu}^{T}(\psi) n^{\mu}=-1$ for all times $\tau \geq 0$. This means that $\int_{\{t=\tau\} \cap\{\text { ergoregion }\}} J_{\mu}^{T}(\psi) n^{\mu}=-1$ for any $\tau \geq 0$, and hence the local energy of $\psi$ over any compact spatial subset containing the ergoregion will not decay at all.

In addition to the violation of any decay statement, the presence of a large ergoregion could even preclude the boundedness of the energy of waves: According to the heuristics of Friedman ([35]), in the case of a stationary, asymptotically flat spacetime with no horizon but with non empty ergoregion, one expects no uniform boundedness statement to hold regarding the energy of solutions to the scalar wave equation. Using the machinery of the proof of Theorem 2.1, we were able to provide a rigorous proof of this instability result, which will be presented in our forthcoming [51]. It is an open

\footnotetext{
${ }^{4}$ Even in that case, however, it may be necessary to weaken Theorem 1.1 to a statement about some degenerate energy current.
} 
problem, however, to understand whether Theorem 1.1 is true in the case Assumption 3 is violated but energy boundedness (namely Assumption 4) still holds.

Finally, even if one assumes the existence of a non empty horizon with positive surface gravity and some form of smallness of the ergoregion, superradiance (i.e. the fact that the domain of outer communications $\mathcal{D}$ of $(\mathcal{M}, g)$ admits no globally non spacelike Killing vector field) may pose serious risks for the boundedness of the energy of waves: Shlapentokh-Rothman (see [63]) has constructed exponentially growing solutions to the Klein-Gordon equation $\square_{g} \psi-m \psi=0$ on a slowly rotating Kerr background, for arbitrarily small values of the mass $m$. For the case of the scalar wave equation on Kerr, energy boundedness can also be violated if a compactly supported real and positive potential is added; see our forthcoming [50]. These facts suggest that Assumption 4 on the boundedness of the energy of scalar waves, which plays a fundamental role in the proof of Theorem 1.1, can not be inferred from the rest of the Assumptions 1-3, and thus should be indisposable if one is not willing to further restrict the class of spacetimes under consideration.

\subsection{Examples}

Beyond the class of asymptotically flat Lorentzian manifolds of product type $(\mathbb{R} \times$ $\mathcal{N},-d t^{2}+\bar{g}$ ), which were already treated by Rodnianski and Tao in [58], there is a large number of other interesting classes of spacetimes on which Theorem 2.1 readily applies to give a novel logarithmic decay statement for the local energy of waves:

- The general class of asymptotically flat non-extremal black hole spacetimes without ergoregion, for which a non degenerate energy boundedness statement has been established by Dafermos and Rodnianski in Section 7 of [22]. This class includes the spherically symmetric black hole spacetimes studied in [47], and in particular includes the black hole solutions of the $S U(2)$ Einstein-Yang Mills equations constructed in [65].

- When restricted to axisymmetric solutions of $\square_{g} \psi=0$, Theorem 2.1 also applies on the domain of outer communications of the higher dimensional EmparanReall black rings (see [33,34]) and the Elvang-Figueras black Saturn (see [32]). Restriction to axisymmetry in this case is necessitated by the fact that no energy boundedness statement (in the spirit of Assumption 4) for general solutions to $\square_{g} \psi=0$ has been established so far on these spacetimes.

- The class of $C^{1}$ perturbations of the slowly rotating Kerr spacetime exterior for which a non degenerate energy boundedness statement was proven by Dafermos and Rodnianski in [21]. Notice that since these perturbations are only close to Kerr exterior in a suitable $C^{1}$ norm, the structure of trapping (which would be controlled by the smallness of a $C^{2}$ norm) can differ substantially on these spacetimes compared to trapping on Kerr exterior. Moreover, note that these spacetimes can possibly contain an ergoregion.

- Keir's class of static spherically symmetric spacetimes, including a class of ultracomact neutron stars (see [44]). Of course, on these spacetimes [44] has already established a logarithmic decay bound, showing also that it is sharp. Thus, these 
spacetimes also serve to exhibit the sharpness of Theorem 2.1 on the class of spacetimes under consideration.

The above examples will be discussed in more detail in Section 2.2, after a more precise statement of Assumptions 1-4 in Section 2.1.

\section{Statement of the Theorem and Sketch of the Proof}

We will now proceed to rigorously state and explain the various geometric assumptions on the class of spacetimes under consideration, and provide the precise statement of the main result of this paper. We will then briefly sketch the proof of this result, highlighting the important ideas involved in the detailed proof occupying the subsequent sections of the paper.

\subsection{Geometric Assumptions on the Background Spacetime}

For the following, let $\left(\mathcal{M}^{d+1}, g\right)$ be a smooth, time oriented, globally hyperbolic $d+1$ dimensional Lorentzian manifold for $d \geq 3$. Let also $\Sigma$ be a smooth spacelike Cauchy hypersurface of $(\mathcal{M}, g)$. We will now formulate assumptions $1-4$ in more detail in terms of the geometry of $(\mathcal{M}, g)$. For a detailed description of the notational conventions adopted in what follows, see Section 3.

Since in our formulation of Assumptions 1-4 we have strived to include a broad class of globally hyperbolic Lorentzian manifolds with as little structure as possible, we will need to perform from scratch some geometric constructions that are trivial in many concrete examples of spacetimes that appear in the literature. To this end, the reader who is only interested in applications of the results of this paper in specific examples of spacetimes should feel free to skip many of the details of the explanation of the constructions performed in the next paragraphs.

\subsubsection{Assumption 1 (Asymptotic Flatness and Stationarity)}

We assume that there exists a smooth Killing vector field $T$ on $\mathcal{M}$, which has complete orbits $^{5}$ and which, when restricted on $\Sigma$, becomes future directed and timelike outside a compact subset of $\Sigma$. We will refer to $T$ as the stationary Killing field.

We also assume that there exists a compact subset $K$ of $\Sigma$ such that $\Sigma \backslash K$ has a finite number of connected components $\left\{\mathcal{S}_{i}\right\}_{i=1}^{m}$, and each connected component $\mathcal{S}_{i}$ of $\Sigma \backslash K$ can be diffeomorphically mapped to $\mathbb{R}^{d} \backslash B_{R}(0)$ (where $B_{R}(0)$ is the ball of $\mathbb{R}^{d}$ of radius $R$ centered at the origin). We will set

$$
\mathcal{S} \doteq \Sigma \backslash K=\cup_{i=1}^{m} \mathcal{S}_{i}
$$

In the resulting chart $\left(x^{1}, x^{2}, \ldots, x^{d}\right)$ on each of the $\mathcal{S}_{i}$ 's, we let $r=$ $\sqrt{\left(x^{1}\right)^{2}+\left(x^{2}\right)^{2}+\ldots+\left(x^{d}\right)^{2}}$ be the pullback of the polar distance on $\mathbb{R}^{d}$. See Sec-

\footnotetext{
5 Namely starting from any point of $\mathcal{M}$ we can follow the flow of $T$ for infinite time in both directions.
} 
tion 3 for the $(r, \sigma)$ notation used for the polar coordinate chart on each connected component of $\mathcal{S}$.

Without loss of generality, we assume that the compact subset $K$ was chosen large enough so that the restriction of $T$ on $\mathcal{S}$ is strictly timelike. Then the integral curves of $T$ would intersect $\mathcal{S}$ transversally. Letting $\mathcal{S}_{\tau}$ be the image of $\mathcal{S}$ under the flow of $T$ for time $\tau$, we deduce that the set

$$
\mathcal{I}_{a s} \doteq \cup_{\tau \in \mathbb{R}} \mathcal{S}_{\tau} \simeq \mathbb{R} \times \mathcal{S}
$$

is an open subset of $(\mathcal{M}, g)$.

We define the coordinate function $t: \mathcal{I}_{a s} \rightarrow \mathbb{R}$ by the relation

$$
\left.t\right|_{\mathcal{S}}=0, T(t)=1
$$

and we note that if we extend the coordinate functions $(r, \sigma): \mathcal{S} \rightarrow \mathbb{R}_{+} \times \mathbb{S}^{d-1}$ (see Section 3 for the $\sigma$ notation) in the whole of $\mathcal{I}_{a s}$ by the conditions $\operatorname{Tr}=0, T \sigma=0$, then $(t, r, \sigma)$ is a valid coordinate chart on each connected component of $\mathcal{I}_{a s}$. In this chart, $\partial_{t}=T$.

Quantitative asympotics for the metric: In the $(t, r, \sigma)$ coordinates oneach connected component of $\mathcal{I}_{a s}$, there exists an $a \in(0,1]$ such that the metric $g$ takes the asymptotically flat form

$$
\begin{aligned}
g= & -\left(1-\frac{2 M}{r}+h_{1}(r, \sigma)\right) d t^{2} \\
& +\left(1+\frac{2 M}{r}+h_{2}(r, \sigma)\right) d r^{2}+r^{2} \cdot\left(g_{\mathbb{S}^{d-1}}+h_{3}(r, \sigma)\right)+h_{4}(r, \sigma) d t d \sigma
\end{aligned}
$$

where:

- $h_{1}, h_{2}$ are smooth $O_{4}\left(r^{-1-a}\right)$ functions. See Section 3 for the $O_{k}(\cdot)$ notation.

- $h_{4}$ is a smooth $O_{4}\left(r^{-a}\right)$ function.

- For every $r, h_{3}(r, \cdot)$ is a smooth symmetric $(0,2)$-tensor field defined on $\mathbb{S}^{d-1}$, and satisfies the bound $h_{3}=O_{4}\left(r^{-1-a}\right)$.

In the above expression for the metric in the asymptotically flat region, we will refer to the constant $M$ as the mass of the spacetime $(\mathcal{M}, g)$, although it will only coincide with the ADM mass of the spacetime if the space dimension is $d=3$.

Notice that (2.4) implies that $T$ has been normalised so that $g(T, T) \rightarrow-1$ as $r \rightarrow \infty .^{6}$

\footnotetext{
${ }^{6}$ One could point out that a general asymptotically flat metric should also contain a $d t \cdot d r$ component, but if this component has $O_{4}\left(r^{-1-a}\right)$ asymptotics, it can always be annihilated for $\{r \gg 1\}$ by a transformation of the form $t \rightarrow t+f(r, \sigma)$, which would of course change our choice of Cauchy hypersurface $\Sigma$ corresponding to $\{t=0\}$. The same is true for the $d r \cdot d \sigma$ terms that might possibly exist in a more general expression of an asymptotically flat metric: If the $d r \cdot d \sigma$ components are $O_{5}\left(\frac{1-a}{2}\right)$, then one can choose a new spherical parametrization $\sigma_{\text {new }}=\sigma_{\text {new }}(r, \sigma)$, so that in $\left(t, r, \sigma_{\text {new }}\right)$ coordinates and for $r \gg 1$ the metric has the form 2.4 .
} 
Some special subsets of $(\mathcal{M}, g)$ : We will define a few subsets of $(\mathcal{M}, g)$ which are tied to the causal structure of the spacetime, and will be frequently referred to throughout the paper.

We define the domain of outer communications of the asymptotically flat end $\mathcal{S}$ of $\Sigma$ to be the subset

$$
\mathcal{D} \doteq \operatorname{clos}\left(J^{-}\left(\mathcal{I}_{a s}\right) \cap J^{+}\left(\mathcal{I}_{a s}\right)\right)
$$

of $\mathcal{M}$. Here, $J^{+}(A)$ denotes the causal future of the set $A,{ }^{7}$ and $J^{-}(A)$ the causal past. If $\mathcal{D}$ is not the whole of $\mathcal{M}$, then the set

$$
\mathcal{H} \doteq \partial \mathcal{D}
$$

will be non empty, and we will call this set the horizon of $(\mathcal{M}, g)$. The horizon $\mathcal{H}$ can be split naturally as the union $\mathcal{H}^{+} \cup \mathcal{H}^{-}$, where $\mathcal{H}^{+} \doteq J^{+}\left(\mathcal{I}_{\text {as }}\right) \cap \partial\left(J^{-}\left(\mathcal{I}_{\text {as }}\right)\right)$ and $\mathcal{H}^{-} \doteq J^{-}\left(\mathcal{I}_{a s}\right) \cap \partial\left(J^{+}\left(\mathcal{I}_{a s}\right)\right)$. Note that $\mathcal{H}^{-}$lies in the past of $\mathcal{H}^{+}$.

If non empty, we will assume that $\mathcal{H}^{+}, \mathcal{H}^{-}$are smooth null hypersurfaces of $(\mathcal{M}, g)$, possibly with boundary $\mathcal{H}^{+} \cap \mathcal{H}^{-}$. Since $T$ is a Killing field of $\mathcal{M}$, and $\mathcal{I}_{\text {as }}$ is by definition invariant under the flow of $T$, we conclude that $\mathcal{D}, \mathcal{H}^{+}, \mathcal{H}^{-}, \mathcal{H}^{+} \cap \mathcal{H}^{-}$and $\mathcal{H}$ must be invariant under the flow of $T$. Since $\mathcal{H}^{+}, \mathcal{H}^{-}$were assumed to be smooth hypersurfaces with boundary, this means that $T$ is tangent to them, and tangent to their boundary.

Notice also that $\mathcal{D}$ might have more than one connected comonents, but it is possible for more than one connected components of $\mathcal{I}_{\text {as }}$ to lie in the same component of $\mathcal{D}$.

Requirements regarding the Cauchy hypersurface $\Sigma$ : We will need to guarantee that the Cauchy hypersurface $\Sigma$ is in the right position so that we can uniquely solve the wave equation $\square_{g} \psi=0$ on $J^{+}(\Sigma) \cap \mathcal{D}$, given initial data on $\Sigma \cap \mathcal{D}$. This will inevitably lead to a few extra hypotheses regarding the part of the Cauchy hypersurface that does not lie in the asymptotically flat region $\mathcal{I}_{a s}$.

We assume that, by altering $\Sigma$ if necessary (but keeping $\Sigma$ as before in the region $\{r \gg 1\})$, we can arrange so that $\mathcal{H}^{-} \subset I^{-}(\Sigma \cap \mathcal{D})^{8}$. With this assumption, for any $p \in J^{+}(\Sigma) \cap \mathcal{D}$ we have $J^{-}(p) \cap \Sigma \subseteq \Sigma \cap \mathcal{D}$ (see Lemma 12.2 for a proof of this fact), and this inclusion implies, according to the arguments of [57], that any smooth solution to $\square_{g} \psi=0$ on $J^{+}(\Sigma) \cap \mathcal{D}$ is uniquely specified by $\left.\left(\psi, n_{\Sigma} \psi\right)\right|_{\Sigma \cap \mathcal{D}}, n_{\Sigma}$ denoting the future directed unit normal to $\Sigma$.

Transversality assumptions on the stationary vector field T: It would be convenient for us to work in coordinate charts where $T$ is a coordinate vector field, and for this reason we have to state two more assumptions regarding the orbits of $T$.

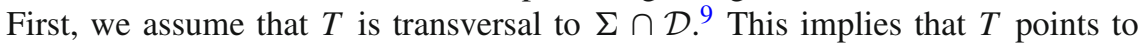
the future of $\Sigma \cap \mathcal{D},{ }^{10}$ in the sense that following the integral curves of $T$ for some

\footnotetext{
7 i.e. the set of points of $\mathcal{M}$ which can be the endpoints of future directed causal curves in $\mathcal{M}$ starting from $A$.

8 i.e. the timelike past of $\Sigma \cap \mathcal{D}$.

9 Note that for this to be true in the Schwarzschild spacetime, for instance, we must choose $\Sigma$ so that it does not intersect the bifurcate sphere.

10 Note that $\mathrm{T}$ may fail to be timelike!
} 
arbitrarily small positive time, starting from apoint on $\Sigma \cap \mathcal{D}$, we are led to a point in $I^{+}(\Sigma \cap \mathcal{D}) .{ }^{11}$

Therefore, the image of $\Sigma \cap \mathcal{D}$ under the flow of $T$ for positive times always lies in $J^{+}(\Sigma) \cap \mathcal{D}$. At this point, we also need to introduce a second assumption on $T$, namely that these images actually cover the whole of $J^{+}(\Sigma) \cap \mathcal{D}$. Given this, one can extend the function $t$ defined before on $\mathcal{I}_{a s}$ to the whole of $J^{+}(\Sigma) \cap \mathcal{D}$, by demanding as before that $\left.t\right|_{\Sigma}=0$ and $T(t)=1$.

By following the flow of $T$ for negative times, $t$ is also defined on the past images of $\Sigma \cap \mathcal{D}$ under the flow of $T$. In this way, $t$ will be defined only on $\mathcal{D} \backslash \mathcal{H}^{-}$and not on the whole of $\mathcal{D}$ (this will not be a problem to us, since we will perform our analysis only on $\left.J^{+}(\Sigma) \cap \mathcal{D}\right)$. We will also denote $\Sigma_{\tau} \doteq\{t=\tau\}$, and with this notation we will have $\Sigma_{0}=\Sigma \cap \mathcal{D}$.

With these assumptions on $T$, if $x=\left(x^{1}, x^{2}, \ldots, x^{d}\right)$ is a local chart on a subset $\mathcal{V}$ of $\Sigma \cap \mathcal{D}$, then extending its coordinates functions $x^{i}$ by the requirement $T\left(x^{i}\right)=0$ one can construct a local coordinate chart $\left(t, x^{1}, x^{2}, \ldots, x^{d}\right)$ on $\mathcal{D}$. In such a local chart, one has $\partial_{t}=T$. We will mostly work in local charts of this form.

Implications for $\Sigma \cap \mathcal{H}$ : Due to our previous assumption that $\mathcal{H}^{-} \subset I^{-}(\Sigma \cap \mathcal{D})$, we deduce that $(\Sigma \cap \mathcal{D}) \cap \mathcal{H}^{-}=\varnothing$ and hence also $(\Sigma \cap \mathcal{D}) \cap\left(\mathcal{H}^{+} \cap \mathcal{H}^{-}\right)=\emptyset$. This, together with the fact that $\Sigma$ intersects $\mathcal{H}^{+}$transversally (since $T$ is tangent to $\mathcal{H}^{+}$ and transversal to $\Sigma$ ) implies that $\Sigma \cap \mathcal{H}^{+}$is a smooth submanifold of $\Sigma$. Since $\mathcal{H}^{+}$ is closed (due to its definition), $\Sigma \cap \mathcal{H}^{+}$is a closed subset of $\Sigma \cap \mathcal{D}$. In fact, $\Sigma \cap \mathcal{H}^{+}$ is the boundary of the manifold-with-boundary $\Sigma \cap \mathcal{D}$. Moreover, $\mathcal{H}$ can not intersect the asymptotically flat region $\mathcal{I}_{a s},{ }^{12}$ and hence $\Sigma \cap \mathcal{H}^{+}$lies in a compact subset of $\Sigma$. Therefore, we conclude $\Sigma \cap \mathcal{H}^{+}$is a compact submanifold of $\Sigma$.

Since $\mathcal{H}^{+}$is $T$-invariant and the future translates of $\Sigma \cap \mathcal{D}$ cover $J^{+}(\Sigma) \cap \mathcal{D}$, we deduce that the future translates of $\mathcal{H}^{+} \cap \Sigma$ by $T$ cover $J^{+}(\Sigma) \cap \mathcal{H}=J^{+}(\Sigma) \cap \mathcal{H}^{+}$. Since $T$ is transversal to $\Sigma \cap \mathcal{H}^{+}$, this implies that $J^{+}(\Sigma) \cap \mathcal{H} \simeq \mathbb{R} \times\left(\Sigma \cap \mathcal{H}^{+}\right)$ is a smooth submanifold of $\mathcal{D}$ with boundary $\Sigma \cap \mathcal{H}^{+}$. Since $\mathcal{H}^{+} \backslash\left(\mathcal{H}^{+} \cap \mathcal{H}^{-}\right)$was assumed to be a null hypersurface, $J^{+}(\Sigma) \cap \mathcal{H}$ must also be a null hypersurface if non empty.

Extension of the function $r$ : We can extend the function $r$ defined before on $\mathcal{S}$ (as the pullback of the polar distance on $\mathbb{R}^{d}$ on each component of $\mathcal{S}$ ) to the whole of $\Sigma \cap \mathcal{D}$, by simply demanding that it is a Morse function on $\Sigma \cap \mathcal{D}$, satisfying $r \geq 0$ on $\Sigma \cap \mathcal{D}$ and with $r=0$ only on $\Sigma \cap \mathcal{H}^{+}$(this is possible since we showed that $\Sigma \cap \mathcal{H}^{+}$is a smooth compact submanifold of $\Sigma$, and at the same time it is the boundary of the manifold with boundary $\Sigma \cap \mathcal{D}$ ). Moreover, we demand that $\left.d r\right|_{\Sigma \cap \mathcal{H}^{+}} \neq 0$, so that $r$ can also be used as a coordinate function close to the horizon.

11 Proof of the last claim: if this claim was false, and $A \subseteq \Sigma \cap \mathcal{D}$ was the set of points of $\Sigma \cap \mathcal{D}$ starting from which the flow of $T$ did not lead to $I^{+}(\Sigma)$ for small times, then $\partial A \neq \emptyset$ (since $A$ was assumed to be non empty, but $A$ does not contain any point in the asymptotically flat region $\{r \gg 1\}$ ). But then, due to the smoothness of $T$, at the points of $\partial A T$ should be tangent to $\Sigma$, which contradicts the assumption of the transversality of $\mathcal{D} \cap \Sigma$ and $T$.

${ }^{12}$ Since $\mathcal{H}=\partial \mathcal{D}$ and $\mathcal{D}=J^{-}\left(\mathcal{I}_{a s}\right) \cap J^{+}\left(\mathcal{I}_{a s}\right)$. 
We can then extend $r$ to the whole of $\mathcal{D}$ by just demanding that $T r=0$. Of course, we might not be able to make $r$ a coordinate function, since if $\Sigma$ has complicated topology, Morse theory implies that $\nabla r$ should necessarily vanish somewhere on $\Sigma$.

Hyperboloidal hypersurfaces terminating at $\mathcal{I}^{+}$: We will need to define a special class of hypersurfaces that will be used frequently throughout this paper.

Let $S$ be a spacelike hypersurface of $\mathcal{D}$. We will say that $S$ is a hyperboloidal hypersurface terminating at $\mathcal{I}^{+}$, if in each component of the asymptotically flat region $\mathcal{I}_{a s}$ of $(\mathcal{D}, g)$, equipped with the coordinate chart $(t, r, \sigma) \in \mathbb{R} \times\left(\mathbb{R}^{d} \backslash B_{R}\right), S$ is the set $\{(t, r, \sigma) \mid t=f(r, \sigma)\}$ for some function $f: \mathbb{R}^{d} \backslash B_{R} \rightarrow \mathbb{R}$ such that

$$
\sup _{(r, \sigma) \in \mathbb{R}^{d} \backslash B_{R}}(|f(r, \sigma)-r-2 M \log (r-2 M)|)<+\infty
$$

\subsubsection{Assumption 2 (Killing Horizon with Positive Surface Gravity)}

In the case $J^{+}(\Sigma) \cap \mathcal{H}^{+} \neq \emptyset$, we assume that there exists a non-zero vector field $V$ defined on $J^{+}(\Sigma) \cap \mathcal{H}^{+}$, which is parallel to the null generators of $J^{+}(\Sigma) \cap \mathcal{H}^{+}$, and its flow preserves the induced (degenerate) metric on $I^{+}(\Sigma) \cap \mathcal{H}^{+}$. We also assume that $V$ commutes with $T$ on $J^{+}(\Sigma) \cap \mathcal{H}$. Moreover, we assume that there exists a $T$ invariant strictly timelike vector field $N$ on $\mathcal{D}$, which, when restricted on $J^{+}(\Sigma) \cap \mathcal{H}$, satisfies

$$
K^{N}(\psi) \geq c J_{\mu}^{N}(\psi) N^{\mu}
$$

for any $\psi \in C^{\infty}(\mathcal{D})$, where $c>0$ is independent of $\psi$. For the notations on currents, see Section 3.8.

We will call the vector field $N$ the red shift vector field. The reason for this name is that a vector field of that form was shown to exist for a general class of Killing horizons with positive surface gravity by Dafermos and Rodnianski in [18]. However, here we will just assume the existence of such a vector field without specifying the geometric orgin of it.

Note that we can modify the vector field $N$ away from the horizon, so that in the region $r \gg 1$ it coincides with $T$, and still retain the bound (2.8) on $J^{+}(\Sigma) \cap \mathcal{H} .{ }^{13} \mathrm{We}$ will hence assume without loss of generality that $N$ has been chosen so that $N \equiv T$ away from the horizon.

Due to the smoothness of $N$, there exists an $r_{0}>0$, such that (2.8) also holds (possibly with a smaller constant $c$ on the right hand side) in a neighborhood of $\mathcal{H}^{+} \backslash\left(\mathcal{H}^{+} \cap \mathcal{H}^{-}\right)$in $\mathcal{D}$ of the form $\left\{r \leq r_{0}\right\}$. For $r \gg 1$, of course, since $N \equiv T$ there, we have $K^{T}(\psi) \equiv 0$. Hence, due to the $T$-invariance of $N$ and the compactness of the sets of the form $\{r \leq R\} \cap \Sigma$, there is also a (possibly large) constant $C>0$ such that

$$
\left|K^{N}(\psi)\right| \leq C \cdot J_{\mu}^{N}(\psi) N^{\mu}
$$

everywhere on $\mathcal{D}$ for any $\psi \in C^{\infty}(\mathcal{D})$.

\footnotetext{
13 The convexity of the cone of the future timelike vectors over each point of $\mathcal{D}$ is used in this argument.
} 
Without loss of generality, we also assume that $r_{0}$ is small enough so that $d r \neq 0$ on $\left\{r \leq 3 r_{0}\right\}$. This is possible, since we have assumed that $\left.d r\right|_{\Sigma \cap \mathcal{H}^{+}} \neq 0$ and that $\Sigma \cap \mathcal{H}^{+}$is compact.

\subsubsection{Assumption 3 (Smallness of the Ergoregion)}

In the case $\mathcal{H}^{+} \neq \emptyset$, we assume that $g(T, T)<0$ in the region $\left\{r>\frac{r_{0}}{2}\right\}$ (where $r_{0}$ was defined before so that $K^{N}(\psi) \geq C \cdot J_{\mu}^{N}(\psi) N^{\mu}$ in $\left.\left\{r \leq r_{0}\right\}\right)$. Moreover, we assume that

$$
\sup \{g(T, T)\}<\varepsilon
$$

for a fixed small positive constant $\varepsilon$, the value of which will be specified exactly later on in terms of the rest of the geometry of our spacetime. ${ }^{14}$ Note that due to the asymptotic flatness assumption, on $\left\{r \geq \frac{3 r_{0}}{4}\right\}$ the quantity $g(T, T)$ is uniformly bounded away from zero.

Since $\{g(T, T)>0\}$ in $\left\{r \geq r_{0}\right\}$, one could have adjusted the previous construction of the vector field $N$ so that it coincides with $T$ on $\left\{r \geq 2 r_{0}\right\}$. Hence, without loss of generality, from now on we will assume that $N \equiv T$ on $\left\{r \geq 2 r_{0}\right\}$.

In the case $\mathcal{H}^{+}=\emptyset$, we assume that $T$ is everywhere timelike on $\mathcal{D}$.

Remark We should remark again that, in view of Friedman's ergospher instability (see [35]), the existence of a non empty ergoregion in the case $\mathcal{H}^{+}=\emptyset$ would lead to a violation of Assumption 4. For a rigorous proof of this result, see our forthcoming [51]. We should also point out that Assumption 3 poses some restrictions on the part of the trapped set contained in the ergoregion. For instance, it does not allow for $T$-orthogonal trapped null geodesics.

\subsubsection{Assumption 4 (Boundedness of the Energy of Scalar Waves)}

Recall that the leaves of the foliation $\left\{\Sigma_{t} \mid t \geq 0\right\}$ of $\mathcal{D} \cap J^{+}(\Sigma)$ are the level sets of the function $t$ defined under Assumption 1. We assume that there exists a positive constant $C>0$ such that for any smooth solution $\psi$ to the wave equation $\square_{g} \psi=0$ on $\mathcal{D} \cap J^{+}(\Sigma)$, and for any $0 \leq t \leq t^{\prime}$, we can bound

$$
\int_{\Sigma_{t^{\prime}}} J_{\mu}^{N}(\psi) n^{\mu} \leq C \cdot \int_{\Sigma_{t}} J_{\mu}^{N}(\psi) n^{\mu},
$$

where $n^{\mu}$ is the unit normal to the leaves of the foliation $\left\{\Sigma_{\tau}\right\}_{\tau \geq 0}$ and the integrals are taken with respect to the volume form of the induced metric.

This assumption will be used as a "black box" in the subsequent sections, in the sense that the mechanisms from which boundedness stems will not be addressed. Notice that we have not included in our assumption the boundedness of the horizon

\footnotetext{
14 Informally, this means that the ergoregion is small enough so that the "red shift" effect can "protect" us from the various difficulties tied to coping with waves inside the ergoregion, such us those stemming from the superradiance effect.
} 
flux $\int_{\mathcal{H}^{+}} J_{\mu}^{N}(\psi) n_{\mathcal{H}}^{\mu}$ (for our conventions on integration over null hypersurfaces, see section 3$)$.

Remark While the boundedness assumption 4 was stated only for the level sets of the function $t$, it actually holds for the level sets of any function of the form $t+\chi$ with achronal level sets, $\chi: \mathcal{D} \rightarrow \mathbb{R}$ being any function supported in $r \gg 1$ and satisfying $T \chi=0$. This fact can be easily deduced by the conservation of the $J_{\mu}^{T}$ current and the fact that $T \equiv N$ is timelike for $r \gg 1$.

\subsection{Examples of Spacetimes Satisfying Assumptions 1-4}

A first example of a broad class of spacetimes satisfying Assumptions 1-4 is the class of asymptotically flat Lorentzian manifolds of product type $\left(\mathbb{R} \times \mathcal{N},-d t^{2}+\bar{g}\right)$, which was already treated by Rodnianski and Tao in [58].

In order to verify that Assumptions 1-4 are indeed satisfied on these spacetimes, one has to notice that Assumption 1 on stationarity and asymptotic flatness follows readily from the asymptotic flatness of the Riemannian manifold $\mathcal{N}$, while Assumptions 2 and 3 are trivially satisfied by the absence of a black hole region and an ergoregion $\left(g\left(\partial_{t}, \partial_{t}\right)\right.$ being identical to -1 everywhere) on product Lorentzian manifolds. Finally, since the vector field $\partial_{t}$ gives rise to the conserved positive definite energy $\int_{\{t=\text { cons }\}} J_{\mu}^{\partial_{t}}(\psi) n_{\{t=\text { const }\}}^{\mu}$ for solutions $\psi$ to the wave equation, Assumption 4 is also satisfied for any Lorentzian manifold of product type.

In addition to the class of asymptotically flat Lorentzian manifolds of product type, some other classes of spacetimes satisfying the assumptions of the previous Section, and on which Theorem 2.1 readily applies to give a logarithmic decay statement for the local energy of waves, are the following:

\subsubsection{Black Hole Spacetimes Without Ergoregion}

In Section 7 of [22], Dafermos and Rodnianski consider a large class of stationary spacetimes without ergoregion, with horizons exhibiting positive surface gravity, and with minimal further geometric and topological assumptions. For these spacetimes, they establish a boundedness estimate for the energy of solutions to the wave equation $\square_{g} \psi=0$ with respect to a timelike vector field and a suitable foliation. Therefore, Assumption 4 on energy boundedness holds for spacetimes in this class.

Restricting our attention to the asymptotically flat spacetimes contained in this class, namely the spacetimes satisfying Assumption 1, the reader can easily verify that they also satisfy Assumptions 2-3 due to the non degeneracy of the horizons and the absence of an ergoregion. Thus, Theorem 2.1 applies in this case to give a logarithmic decay estimate for the local energy of solutions to $\square_{g} \psi=0$. This decay rate for the local energy can also be upgraded to a pointwise logarithmic decay statement for $\psi$, using the commutation techniques explained in [22], thus improving the results of [22] on those spacetimes.

This class includes the spherically symmetric and asymptotically flat black hole spacetimes studied in [47]. An example of spacetimes in the class studied by [47] are 
the black hole solutions of the $S U$ (2) Einstein-Yang Mills equations constructed in [65]. On these spacetimes, our Theorem 2.1 improves the qualitative results of [47].

\subsubsection{Axisymmetric Waves on Black Hole Rings and Black Saturn}

The topological censorship theorem, established by Friedman, Schleich and Witt in [36], implies, through the work of Chruściel and Wald [12] and Jacobson and Venkataramani [42], that in the realm of $3+1$ dimensional vacuum spacetimes, spatial cross sections of black hole horizons must have spherical topology. In higher dimensions, however, horizons are allowed to become more complicated. In $4+1$ dimensions, for instance, the intersection of the black hole horizon with a Cauchy hypersurface in a vacuum spacetime is allowed to have components diffeomorphic to $\mathbb{S}^{1} \times \mathbb{S}^{2}$.

The first exact solutions of the vacuum Einstein equations exhibiting horizons with non spherical topology were the black ring solution constructed by Emparan and Reall in $[33,34]$. These are $4+1$ dimensional, stationary and axisymmetric asymptotically flat solutions to the vacuum Einstein equations, with non degenerate horizons having $\mathbb{S}^{1} \times \mathbb{S}^{2}$ spatial cross sections. A variation of these spacetimes are the black hole rings rotating in two different directions presented by Pomeransky and Sen'kov in [54].

The Emparan-Reall spacetimes necessarily possess an ergoregion, and this fact implies that an energy boundedness statement on their domain of outer communications $\mathcal{D}$ can not be inferred as a corollary of the general result of Dafermos and Rodnianski in [22], discussed in the previous paragraph. However, since in $\mathcal{D}$ the span of the stationary Killing field $T$ and the axisymmetric Killing field $\Phi$ always contains a timelike vector, axisymmetric functions on these spacetimes have positive $T$-energy with respect to any spacelike foliation of $\mathcal{D}$. Thus, axisymmetric solutions to the wave equation $\square \psi=0$ on the domain of outer communications of the aforementioned spacetimes do not "see" the ergoregion, and for them the results of Dafermos and Rodnianski discussed in Section 7 of [22] applies to establish a non degenerate energy boundedness statement.

Therefore, restricting our attention only to axisymmetric waves on $\mathcal{D}$, we can easily verify that all Assumptions 1-4 (reduced, of course, to the case of axisymmetric waves) are satisfied in this case. Furthermore, in view of the fact that $\Phi$ is a Killing field, the proof of Theorem 2.1 can be restricted on the class of axisymmetric functions on $\mathcal{D}$. Thus, we infer that the local energy of axisymmetric solutions to the wave equation $\square_{g} \psi=0$ on the domain of outer communications of the Emparan-Reall spacetimes decay at least logarithmically in time (and this decay rate can also be made pointwise, after implementing as before the commutation techniques explained in [22]).

The situation is similar on the "black Saturn" solution to the vacuum Einstein equations, constructed by Elvang and Figueras in [32]. The Elvang-Figueras spacetime is a 4+1 dimensional, stationary, axisymmetric and asymptotically flat vacuum spacetime, possessing a black hole region with non degenerate horizon. The horizon has spatial cross sections resembling Saturn: one component of the horizon is homeomorphic to $\mathbb{S}^{3}$, while the other one is homeomorphic to $\mathbb{S}^{1} \times \mathbb{S}^{2}$. Arguing exactly as before, the results of Dafermos and Rodnianski presented in [22] establish a non degenerate energy boundedness statement for axisymmetric solutions to the wave equation 
$\square_{g} \psi=0$ on the domain of outer communications $\mathcal{D}_{s}$ of this spacetime. Assumptions 1-4 are also satisfied, therefore, in this case as before, and we deduce that axisymmetric solutions to the wave equation $\square \psi=0$ on $\mathcal{D}_{s}$ decay at least logarithmically in time.

It would be interesting to study in greater detail the axisymmetric trapping on the Elvang-Figueras spacetime (which appears to be a more challenging task due to the horiozn having two components), so as to infer better decay results in this case. Moreover, we should remark that establishing an energy boundedness statement for those of the Emparan-Reall and Elvang-Figueras spacetimes possessing a small ergoregion (which could in principle be achieved by the strategy followed in [21] for slowly rotating Kerr spacetimes, if supperadiance and trapping can be shown to not "overlap") would remove our restriction on axisymmetric waves, making Theorem 2.1 applicable to all solutions of the wave equation on these spacetimes.

\subsection{3 $C^{1}$ Perturbations of Slowly Rotating Kerr Spacetimes}

Of special interest are also the $C^{1}$ perturbations of the exterior of slowly rotating Kerr spacetimes, discussed by Dafermos and Rodnianski in [21]. In particular, these spacetimes $(\mathcal{M}, g)$ possess an atlas, in which the expression for the metric $g$ is $C^{1}$ close to the metric $g_{M}$ expressed on a fixed atlas on the domain of outer communications (including the horizon) of Schwarzschild spacetime $\left(\mathcal{M}_{M}, g_{M}\right)$ with mass $M$. In addition, all spacetimes $(\mathcal{M}, g)$ in this class are required to be stationary and axisymmetric. These spacetimes include the exterior of very slowly rotating Kerr spacetimes (i.e. with $|a| \ll M$ ).

On spacetimes belonging to the above class, it was established in [21] that solutions to the wave equation $\square_{g} \psi=0$ have bounded energy with respect to a globally timelike $T$-invariant vector field $N$ (where $T$ is the stationary Killing field of $(\mathcal{M}, g)$ ) and a suitable spacelike foliation. Therefore, it can be easily checked that Assumptions 1-4 are satisfied for spacetimes belonging to this class, and thus our Theorem 2.1 applies in this case to provide a logarithmic decay rate for the local energy of waves (which, again, can also be upgraded to a pointwise logarithmic decay rate).

It is important to remark that on this class of spacetimes, the nature of the trapped set can be very different from the one in Schwarzschild or slowly rotating Kerr spacetimes, because the metric $g$ of $\mathcal{M}$ was required to be only $C^{1}$ close to that of $\left(\mathcal{M}_{M}, g_{M}\right){ }^{15}$ Hence, methods relying on the particular form of trapping on Schwarzschild or Kerr exterior backgrounds would not be applicable in order to obtain quantitative decay estimates in this class of spacetimes.

\subsubsection{Keir's Class of Spherically Symmetric Spacetimes}

Another class of spacetimes which satisfy Assumptions 1-4 are the static spherically symmetric spacetimes (including spherically symmetric ultracompact neutron stars)

\footnotetext{
15 That is to say, since it is (at least) the $C^{2}$ norm of the difference of the metrics that would control the change in trapping, and this norm is not assumed to be small, the nature of the trapped set in a spacetime $(\mathcal{M}, g)$ of this class can be fundamentally different from the one in Schwarzschild spacetime.
} 
studied by Keir in [44]. Of course, [44] already establishes a logarithmic decay estimate for solutions to the wave equation $\square_{g} \psi=0$, and thus Theorem 2.1 in this case just recovers some of the results of [44]. Since it was shown in [44] that in this class of spacetimes the logarithmic decay rates are optimal, this class also exhibits the optimality of Theorem 2.1. See Section 12 for more on this.

\subsection{Statement of the Decay Result}

We are now able to state the main result of the current paper:

Theorem 2.1 Let $\left(\mathcal{M}^{d+1}, g\right), d \geq 3$, be a globally hyperbolic spacetime with a Cauchy hypersurface $\Sigma$ satisfying Assumptions 1-4 as described in the previous section. Let $\mathcal{D}$ be the domain of outer communications of $(\mathcal{M}, g)$, and let $t: J^{+}(\Sigma) \cap \mathcal{D} \rightarrow[0,+\infty)$ be the time function defined according to Assumption 1. Assume, moreover, that, in case $\mathcal{H}^{+} \neq \emptyset$, the $\varepsilon>0$ appearing in (2.10) is sufficiently small in terms of the geometry of $J^{+}(\Sigma) \cap \mathcal{D} \backslash\{g(T, T)>0\}$. Then for any $R_{1}>0$, any $0<\delta_{0} \leq 1$ and any $m \in \mathbb{N}$, there exists a positive constant $C_{m, \delta_{0}}\left(R_{1}\right)>0$, such that every smooth solution $\psi: J^{+}(\Sigma) \cap \mathcal{D} \rightarrow \mathbb{C}$ to the wave equation $\square_{g} \psi=0$ satisfies the following estimate for any $\tau>0$ :

$$
\begin{aligned}
\int_{\{t=\tau\} \cap\left\{r \leq R_{1}\right\}} J_{\mu}^{N}(\psi) n^{\mu} \leq & \frac{C_{m, \delta_{0}}\left(R_{1}\right)}{\{\log (2+\tau)\}^{2 m}}\left(\sum_{j=0}^{m} \int_{\{t=0\}} J_{\mu}^{N}\left(T^{j} \psi\right) n^{\mu}\right) \\
& +\frac{C_{m}\left(R_{1}\right)}{\tau^{\delta} 0} \int_{\{t=0\}}(1+r)^{\delta_{0}} \cdot J_{\mu}^{N}(\psi) n^{\mu},
\end{aligned}
$$

$n^{\mu}$ being the future directed normal to the leaves of the foliation $\{t=$ const $\}$ of $J^{+}(\Sigma) \cap \mathcal{D}$. See Section 3.8 for the notations on currents.

Remark Theorem 2.1 also yields a pointwise logarithmic decay estimate for $\psi$ under slightly stronger assumptions on $(\mathcal{M}, g)$. In particular, assume that the energy boundedness assumption 4 also holds for higher order energies of $\psi$, and that the following higher order red-shift estimate holds for any $k \geq 1, \tau>0$ :

$$
\begin{aligned}
\sum_{j=0}^{k} \int_{\{0 \leq t \leq \tau\} \cap\left\{r \leq r_{0}\right\}} J_{\mu}^{N}\left(N^{j} \psi\right) n^{\mu} \lesssim_{k} \sum_{j=0}^{k}\left\{\int_{\{0 \leq t \leq \tau\} \cap\left\{r_{0} \leq r \leq 2 r_{0}\right\}} J_{\mu}^{N}\left(N^{j} \psi\right) n^{\mu}\right. \\
\left.+\int_{\{t=0\}} J_{\mu}^{N}\left(N^{j} \psi\right) n^{\mu}\right\}
\end{aligned}
$$

(notice that (2.13) holds on Schwarzschild exterior spacetime, see [18]). Then, the logarithmic decay estimate (2.12) also holds for higher order local energies of $\psi$. Combined with Corollary 2.2, standard elliptic estimates and the Sobolev embedding theorem, these higher order estimates yield a pointwise logarithmic decay estimate for $\psi$ : 


$$
\begin{aligned}
\sup _{\{t=\tau\} \cap\left\{r \leq R_{1}\right\}}|\psi|^{2} \leq & \frac{C_{m}\left(R_{1}\right)}{\{\log (2+\tau)\}^{2 m}} \sum_{i=0}^{\left\lceil\frac{d-1}{2}\right\rceil}\left(\int_{\{t=0\}} r^{\delta 0} J_{\mu}^{N}\left(N^{i} \psi\right) n^{\mu}\right. \\
& \left.+\sum_{j=0}^{m} \int_{\{t=0\}} J_{\mu}^{N}\left(N^{i} T^{j} \psi\right) n^{\mu}\right) .
\end{aligned}
$$

In view of Corollary 2.2, $\sup _{S_{\tau}}|\psi|^{2}$ (where $\left\{S_{\tau}\right\}_{\tau \geq 0}$ is the hyperboloidal foliation of Corollary 2.2) is also controlled by the right hand side of (2.14), with a constant independent of $R_{1}$.

We should also remark that, replacing Assumption 4 with an energy boundedness estimate with loss of derivatives:

$$
\int_{\{t=\tau\}} J_{\mu}^{N}(\psi) n^{\mu} \lesssim \sum_{j=0}^{k} \int_{\{t=0\}} J_{\mu}^{N}\left(N^{j} \psi\right) n^{\mu}
$$

Theorem 2.1 still holds, with (2.12) replaced by:

$$
\begin{aligned}
\int_{\{t=\tau\} \cap\left\{r \leq R_{1}\right\}} J_{\mu}^{N}(\psi) n^{\mu} \leq & \sum_{i=0}^{k}\left\{\frac{C_{m, \delta_{0}}\left(R_{1}\right)}{\{\log (2+\tau)\}^{2 m}}\left(\sum_{j=0}^{m} \int_{\{t=0\}} J_{\mu}^{N}\left(T^{j} N^{i} \psi\right) n^{\mu}\right)\right. \\
& \left.+\frac{C_{m}\left(R_{1}\right)}{\tau^{\delta 0}} \int_{\{t=0\}}(1+r)^{\delta 0} \cdot J_{\mu}^{N}\left(N^{i} \psi\right) n^{\mu}\right\} .
\end{aligned}
$$

\subsection{Corollaries of Theorem 2.1}

As a first corollary of Theorem 2.1, using [52], we will upgrade the statement of Theorem 2.1 itself to a logarithmic decay estimate for the energy of a solution $\psi$ to (1.1) with respect to hyperboloidal hypersurfaces terminating at $\mathcal{I}^{+}$:

Corollary 2.2 (Logarithmic decay of the energy through a hyperboloidal foliation). Let $(\mathcal{M}, g), \Sigma, \mathcal{D}$ and $t$ be as in the statement of Theorem 2.1. Let $S_{0}$ be any smooth hyperboloidal hypersurface terminating at $\mathcal{I}^{+}$(according to the definition under Assumption 1), intersecting transversally $\mathcal{H}^{+} \cap J^{+}(\Sigma)$ (if non empty), such that $S_{0} \subset J^{+}(\{t=0\})$. Let also $S_{t}$ denote the image of $S_{0}$ under the flow of $T$ for time $t>0$. Then, for any $m \in \mathbb{N}$, there exists a positive constant $C_{m}>0$ such that every smooth solution $\psi: J^{+}(\Sigma) \cap \mathcal{D} \rightarrow \mathbb{C}$ to the wave equation $\square_{g} \psi=0$ satisfies the following estimate for any $\tau>0$ :

$$
\int_{S_{\tau}} J_{\mu}^{N}(\psi) n_{S}^{\mu} \leq \frac{C_{m}}{\{\log (2+\tau)\}^{2 m}}\left(\int_{\{t=0\}} r^{\delta 0} J_{\mu}^{N}(\psi) n^{\mu}+\sum_{j=0}^{m} \int_{\{t=0\}} J_{\mu}^{N}\left(T^{j} \psi\right) n^{\mu}\right),
$$

$n_{S}^{\mu}$ being the future directed normal to the leaves of the foliation $S_{\tau}$ and $n^{\mu}$ being the future directed normal to the leaves of the foliation $\{t=$ const $\}$. 
The proof of this corollary is a direct consequence of Theorem 2.1, combined with the $r^{p}$-weighted energy method of [52]. It is presented in Section 10.

The quantitative decay rate (2.17) also allows us to infer that, for solutions $\psi$ to the wave equation which have initial data with only finite initial $J^{N}$-energy (and thus with initial data with not necessarily enough decay in $r$ for Theorem 2.1 to apply), the $J^{N}$-energy of $\psi$ with respect to a foliation of hyperboloidal hypersurfaces terminating at $\mathcal{I}^{+}$still decays to 0 , albeit in a non quantitative manner. In particular, we can establish the following result:

Corollary 2.3 (Non-quantitative hyperboloidal decay in the energy class). Let $(\mathcal{M}, g), \Sigma, \mathcal{D}$ and $t$ be as in the statement of Theorem 2.1 . Let $S_{0}$ be any smooth hyperboloidal hypersurface terminating at $\mathcal{I}^{+}$(according to the definition under Assumption 1 ), intersecting transversally $\mathcal{H}^{+} \cap J^{+}(\Sigma)$ (ifnon-empty), such that $S_{0} \subset J^{+}(\{t=0\})$. Let also $S_{t}$ denote the image of $S_{0}$ under the flow of $T$ for time $t>0$. Then, for every solution $\psi: J^{+}(\Sigma) \cap \mathcal{D} \rightarrow \mathbb{C}$ to the wave equation $\square_{g} \psi=0$ on $(\mathcal{D}, g)$ with $\int_{t=0} J_{\mu}^{N}(\psi) n^{\mu}<\infty, n^{\mu}$ being the future directed normal to the leaves of the foliation $\{t=$ const $\}$, the following is true:

$$
\lim _{\tau \rightarrow+\infty} \int_{S_{\tau}} J_{\mu}^{N}(\psi) n_{S}^{\mu}=0,
$$

$n_{S}^{\mu}$ being the future directed normal to the leaves of the foliation $S_{\tau}$.

If the stationary vector field $T$ is non spacelike in $\mathcal{D}$ and timelike away from the future event horizon $\mathcal{H}^{+}$, then

$$
\lim _{\tau \rightarrow+\infty} \int_{S_{\tau}} J_{\mu}^{T}(\psi) n_{S}^{\mu}=0
$$

also holds for solutions $\psi$ to $\square_{g} \psi=0$ satisfying merely $\int_{t=0} J_{\mu}^{T}(\psi) n^{\mu}<\infty$.

The proof of Corollary 2.3 is presented in Section 11 .

In spacetimes $(\mathcal{M}, g)$ where the stationary vector field $T$ is everywhere non spacelike on the domain of outer communications $\mathcal{D}$, and timelike away from the horizon $\mathcal{H},{ }^{16}$ the qualitative decay statement (2.19) for solutions to the wave equation with finite initial $J^{T}$-energy readily leads to a few interesting scattering results. Before stating these results, we will first define the notion of the future radiation field on the spacetimes under consideration.

Repeating the proof of Theorem 7.1 of [52] for spacetimes $(\mathcal{M}, g)$ satisfying Assumption 1 on asymptotic flatness, ${ }^{17}$ any smooth solution $\psi$ to the wave equation $\square_{g} \psi=0$ on $\mathcal{D}$ (the domain of outer communications of an asymptotically flat end of $\mathcal{M}$ ) with compactly supported initial data on $\Sigma \cap \mathcal{D}$ ( $\Sigma$ being a Cauchy hypersurface of $\mathcal{M}$ according to Assumption 1) gives rise to a well defined radiation field $\Psi_{\mathcal{I}^{+}}$on the future null infinity $\mathcal{I}^{+}$of $(\mathcal{D}, g)$. The future radiation field $\Psi_{\mathcal{I}^{+}}$is defined

\footnotetext{
16 In this case, the $J^{T}$-energy is coercive.

17 The only difference between spacetimes satisfying Assumption 1 and the ones considered in the statement of Theorem 7.1 of [52] is in the regularity of the metric $g$ near $\mathcal{I}^{+}$.
} 
as the limit in the $(u, r, \sigma)$ coordinate system in the region $\{r \gg 1\}$ (see Section 1 of the Appendix for how to construct this coordinate system in a spacetime with the asymptotics (2.4) for the metric) as follows:

Definition For any smooth solution $\psi$ to the wave equation $\square_{g} \psi=0$ on $\mathcal{D}$ with compactly supported initial data on $\Sigma \cap \mathcal{D}$, the future radiation field $\Psi_{\mathcal{I}^{+}}$on $\mathcal{I}^{+}$is defined as the limit (in each connected component of $\mathcal{I}_{a s}$ )

$$
\Psi_{\mathcal{I}^{+}}(u, \sigma)=\lim _{r \rightarrow+\infty} r^{\frac{d-1}{2}} \psi(u, r, \sigma)
$$

where the convergence refers to the $H_{l o c}^{1}\left(\mathbb{R} \times \mathbb{S}^{d-1}, d u^{2}+g_{\mathbb{S}^{d-1}}\right)$ topology.

The $J^{T}$-energy flux of $\psi$ on $\mathcal{I}^{+}$is then defined in the following way:

$$
\int_{\mathcal{I}^{+}} J_{\mu}^{T}(\psi) n_{\mathcal{I}^{+}}^{\mu} \doteq \sum_{\text {components of } \mathcal{I}^{+}} \int_{-\infty}^{\infty} \int_{\mathbb{S}^{d-1}}\left(\partial_{u} \Psi_{\mathcal{I}^{+}}(u, \sigma)\right)^{2} d \sigma d u
$$

A priori, the energy (2.21) might be infinite. However, if the vector field $T$ is everywhere non spacelike on $\mathcal{D}$, and timelike away from the horizon $\mathcal{H}$, then the conservation of the $J^{T}$-current easily implies that

$$
\int_{\mathcal{I}^{+}} J_{\mu}^{T}(\psi) n_{\mathcal{I}^{+}}^{\mu} \leq \int_{t=0} J_{\mu}^{T}(\psi) n^{\mu}
$$

Through the conservation of the $J^{T}$-current one can bound the energy flux on the future horizon by the initial energy of $\psi$. In particular, one can bound:

$$
\int_{\mathcal{H}^{+} \cap\{t \geq 0\}} J_{\mu}^{T}(\psi) n_{\mathcal{H}^{+}}^{\mu}+\int_{\mathcal{I}^{+}} J_{\mu}^{T}(\psi) n_{\mathcal{I}^{+}}^{\mu} \leq \int_{t=0} J_{\mu}^{T}(\psi) n^{\mu}
$$

(see Section 3 on integration over null hypersurfaces).

By a standard density argument, one can define the future radiation field $\Psi_{\mathcal{I}^{+}}$for solutions $\psi$ of $\square_{g} \psi=0$ satisfying merely $\int_{t=0} J_{\mu}^{T}(\psi) n^{\mu}<\infty$ as follows:

Definition For any solution $\psi$ to the wave equation $\square_{g} \psi=0$ on $\mathcal{D}$ with $H_{l o c}^{1}$ initial data on $\Sigma \cap \mathcal{D}$ satisfying $\int_{t=0} J_{\mu}^{T}(\psi) n^{\mu}<\infty$, the future radiation field $\Psi_{\mathcal{I}^{+}}$is defined as the limit (in the topology defined by the energy norm (2.21))

$$
\Psi_{\mathcal{I}^{+}}=\lim _{n} \Psi_{n, \mathcal{I}^{+}},
$$

where $\Psi_{n, \mathcal{I}^{+}}$is the future radiation field of a sequence of smooth functions $\psi_{n}$ solving $\square_{g} \psi_{n}=0$ on $\mathcal{D}$ with $\left.\left(\psi_{n}, T \psi_{n}\right)\right|_{\Sigma \cap \mathcal{D}}$ compactly supported and approximating $\left.(\psi, T \psi)\right|_{\Sigma \cap \mathcal{D}}$ in the $\int_{t=0} J_{\mu}^{T}(\cdot) n^{\mu}$ norm as $n \rightarrow+\infty$. 
The existence and uniqueness of $\Psi_{\mathcal{I}^{+}}$in this case follows by applying (2.23) for the sequence $\psi_{n+1}-\psi_{n}$.

The qualitative decay statement for the $J^{T}$-energy on hyperboloids provided by Corollary 2.3 leads to the following scattering result:

Corollary 2.4 (Asymptotic completeness for spacetimes without ergoregion) Let $(\mathcal{M}, g), \Sigma, \mathcal{D}$ and $t$ be as in the statement of Theorem 2.1, and moreover suppose that the stationary vector field $T$ on $\mathcal{D}$ is everywhere non spacelike, and timelike away from the horizon $\mathcal{H}$. Then, for every solution $\psi: J^{+}(\Sigma) \cap \mathcal{D} \rightarrow \mathbb{R}$ to the wave equation $\square_{g} \psi=0$ on $(\mathcal{D}, g)$ with $\int_{t=0} J_{\mu}^{T}(\psi) n^{\mu}<\infty, n^{\mu}$ being the future directed normal to the leaves of the foliation $\{t=$ const $\}$, inequality (2.23) is actually an equality:

$$
\int_{\mathcal{H}^{+} \cap\{t \geq 0\}} J_{\mu}^{T}(\psi) n_{\mathcal{H}^{+}}^{\mu}+\int_{\mathcal{I}^{+}} J_{\mu}^{T}(\psi) n_{\mathcal{I}^{+}}^{\mu}=\int_{t=0} J_{\mu}^{T}(\psi) n^{\mu}
$$

Furthermore, for any scattering data set $\left(\left.\psi\right|_{\mathcal{H}^{+}}, \Psi_{\mathcal{I}^{+}}\right)$with $\int_{\mathcal{H}^{+}} J_{\mu}^{T}(\psi) n_{\mathcal{H}^{+}}^{\mu}+$ $\int_{\mathcal{I}^{+}} J_{\mu}^{T}(\psi) n_{\mathcal{I}^{+}}^{\mu}<\infty$, one can find a solution $\psi$ to the wave equation on $\mathcal{D}$ with $\int_{t=0} J_{\mu}^{T}(\psi) n^{\mu}<\infty$, for which the restriction on $\mathcal{H}^{+}$and the resulting future radiation field coincide with the given scattering data.

Thus, any solution $\psi$ to $\square_{g} \psi=0$ with $\int_{t=0} J_{\mu}^{T}(\psi) n^{\mu}<\infty$ on $\mathcal{D} \cap J^{+}(\Sigma)$ is uniquely determined by its scattering data on $\mathcal{H}^{+}, \mathcal{I}^{+}$(i.e. by $\left.\psi\right|_{\mathcal{H}^{+}}, \Psi_{\mathcal{I}^{+}}$).

Remark Let $(\mathcal{M}, g)$ and $\mathcal{D}$ be as in the statement of Theorem 2.1 (now $(\mathcal{D}, g)$ is allowed to have a non-empty ergoregion), and assume that $(\mathcal{M}, g)$ is also axisymmetric with axisymmetric Killing field $\Phi$. If the span of $\{T, \Phi\}$ is timelike everywhere on $\mathcal{D} \backslash \mathcal{H}$, then Corollary 2.4 is valid when restricted to the class of axisymmetric solutions to $(1.1)$ on $(\mathcal{M}, g)$.

The proof of Corollary 2.4 will be furnished in Section 11.

Let us notice that Corollary 2.4 reproves the classical asymptotic completeness result on Schwarzschild spacetime (see [26-28,53]), and applies on a large class of asymptotically flat spacetimes, including, for example, the black hole exterior spacetimes constructed in Section 7 of [22], the black hole solutions of the $S U$ (2) Einstein-Yang Mills equations of [65] and the spherically symmetric spacetimes studied by Keir in [44]. Furthermore, restricted on the class of axisymmetric solutions of equation (1.1) (see the remark below Corollary 2.4), it also applies on the subsextremal Kerr exterior, the Emparan-Reall black ring (see [33,34]) and the Elvang-Figueras black Saturn (see [32]). These spacetimes were discussed in Section 2.2.

Notice, however, that Corollary 2.4 does not apply on the general class of solutions to (1.1) on spacetimes with an ergoregion, and, thus, it can not reprove the results of [24] on the subextremal Kerr family (with Kerr parameters in the full subextremal range $|a|<M$ ) without axisymmetry.

Finally, let us remark that in the asymptotically flat region of the spacetimes $(\mathcal{M}, g)$ on which Corollary 2.4 applies, the long range part of the metric $g$ (viewed as a 
perturbation of the flat Lorentzian metric $\eta)$ is spherically symmetric. ${ }^{18}$ The rest of the results of the present paper can be relaxed to apply on stationary spacetimes $(\mathcal{M}, g)$ with $g$ having a non spherically symmetric long range part; however, the proof of the asymptotic completeness statement of Corollary 2.4 would fail to generalise in that case.

\subsection{Sketch of the Proof of Theorem 2.1}

In order to make the reasoning behind the subsequent proof of Theorem 2.1 more transparent, we will first present a sketch of this proof on a heuristic level. The final step of the proof of Theorem 2.1 is the implementation of a frequency interpolation scheme, similar to the one appearing already in [39].

Frequency decomposition: Given any solution $\psi$ to the wave equation $\square_{g} \psi=0$ as in the statement of Theorem 2.1, one starts by splitting it into two parts, $\psi_{\leq \omega_{+}}$and $\psi_{\geq \omega_{+}}$, where $\psi_{\leq \omega_{+}}$has frequency support with respect to the $t$ coordinate in $\left\{|\omega| \leq \omega_{+}\right\}$, and similarly $\psi_{\geq \omega_{+}}$has frequency support contained in $\left\{|\omega| \geq \omega_{+}\right\}$. The parameter $\omega_{+}>0$ will be determined later.

Since the Killing vector field $T$ is not necessarily timelike near $\mathcal{H}^{+}$and we have not assumed that the red-shift vector field $N$ satisfies some "nice" commutation properties with the wave operator $\square_{g}$, the boundedness assumption for the energy of $\psi$ alone is not even enough to exclude the pointwise exponential growth of $\psi$. Thus, we will need to perform a suitable cut-off in physical space in order for the Fourier transform of $\psi$ in $t$ to make sense. Such a cut-off gives rise to well understood error terms in all the subsequent estimates (these issues being conceptually identical to the ones appearing in [21]), and the treatment of these terms will be carried out in a fashion similar to [21] in Section 4.1. The boundedness assumption 4 will play a crucial role in estimating these error terms. But for the sake of simplicity of the current sketch of the proof, let us momentarily ignore the need for a physical space cut-off with all its corresponding implications.

The interpolation argument: The components $\psi_{\geq \omega_{+}}$and $\psi_{\leq \omega_{+}}$of $\psi$ also satisfy the wave equation, due to the stationarity of the metric and the fact that a frequency cut-off corresponds to convolution with a function of $t$ in physical space. One can hence use the boundedness assumption 4 for the high frequency part $\psi_{\geq \omega_{+}}$to conclude that for any $\tau>0$ :

$$
\int_{\{t=\tau\} \cap\left\{r \leq R_{1}\right\}} J_{\mu}^{N}\left(\psi_{\geq \omega_{+}}\right) n^{\mu} \leq C \cdot \int_{\{t=0\}} J_{\mu}^{N}(\psi) n^{\mu} .
$$

(Notice that the right hand side of (2.26) contains the energy of $\psi$ and not that of $\psi_{>\omega_{+}}$, which is a result of the technical issues concerning the physical space cut-off and the frequency decomposition that we will supress in this section). Since the vector field $T$ is Killing, (2.26) also holds for the pair $T^{m} \psi_{\geq \omega_{+}}, T^{m} \psi$ in place of $\psi_{\geq \omega_{+}}, \psi$, and thus, due to the fact that the frequency support of $\psi_{\geq \omega_{+}}$is contained in $\left\{|\omega| \geq \omega_{+}\right\}$,

\footnotetext{
18 Notice, however, that the proof of Corollary 2.4 can be adapted to include metrics $g$ for which the long range part in the outgoing null directions is not spherically symmetric.
} 
one can prove a statement of the form

$$
\int_{\{t=\tau\} \cap\left\{r \leq R_{1}\right\}} J_{\mu}^{N}\left(\psi_{\geq \omega_{+}}\right) n^{\mu} \leq \frac{C}{\omega_{+}^{2 m}} \sum_{j=0}^{m} \int_{\{t=0\}} J_{\mu}^{N}\left(T^{j} \psi\right) n^{\mu}
$$

As for the low frequency part $\psi_{\leq \omega_{+}}$, we will show that its local energy decays in time at a slow polynomial rate:

$$
\int_{\{t=\tau\} \cap\left\{r \leq R_{1}\right\}} J_{\mu}^{N}\left(\psi_{\leq \omega_{+}}\right) n^{\mu} \leq \frac{C \cdot e^{C \omega_{+}}}{\tau^{\delta 0}} \int_{\{t=0\}} J_{\mu}^{N}(\psi) n^{\mu}+\frac{C}{\tau^{\delta 0}} \int_{\{t=0\}} r^{\delta 0} \cdot J_{\mu}^{N}(\psi) n^{\mu} .
$$

Notice already the exponential dependence of the constant of the first term of the right hand side of (2.28) on $\omega_{+}$. Assuming for a moment that (2.28) holds, the logarithmic decay of the local energy of $\psi$ is deduced as follows: Combining (2.27) with (2.28), and recalling that $\psi=\psi_{\leq \omega_{+}}+\psi_{\geq \omega_{+}}$, we obtain for any $\tau>0$

$$
\begin{aligned}
\int_{\{t=\tau\} \cap\left\{r \leq R_{1}\right\}} J_{\mu}^{N}(\psi) n^{\mu} \leq & \frac{C \cdot e^{C \omega_{+}}}{\tau \delta 0} \int_{\{t=0\}} J_{\mu}^{N}(\psi) n^{\mu}+\frac{C}{\tau^{\delta 0}} \int_{\{t=0\}} r \cdot J_{\mu}^{N}(\psi) n^{\mu} \\
& +\frac{C}{\omega_{+}^{2 m}} \sum_{j=0}^{m} \int_{\{t=0\}} J_{\mu}^{N}\left(T^{j} \psi\right) n^{\mu}
\end{aligned}
$$

Thus, choosing $\omega_{+} \sim \frac{1}{2 C} \log (2+\tau)$ yields (if $\tau \geq 1$ ) the desired logarithmic decay estimate:

$$
\begin{aligned}
\int_{\{t=\tau\} \cap\left\{r \leq R_{1}\right\}} J_{\mu}^{N}(\psi) n^{\mu} \leq & \frac{C}{\{\log (2+\tau)\}^{2 m}}\left(\sum_{j=0}^{m} \int_{t=0} J_{\mu}^{N}\left(T^{j} \psi\right) n^{\mu}\right) \\
& +\frac{C}{\tau^{\delta 0}} \int_{t=0} r^{\delta 0} \cdot J_{\mu}^{N}(\psi) n^{\mu} .
\end{aligned}
$$

Remark In this interpolation procedure it is evident that, exactly as expected, the "slow" log decay is caused by the high frequency part of $\psi$, since the low frequency one decays polynomially. Such an interpolation scheme was also used in [39] (see also [8]).

Thus, the proof will be complete after establishing a uniform decay statement of the form (2.28) for the low frequency part $\psi_{\leq \omega_{+}}$.

Polynomial decay for $\psi_{\leq \omega_{+}}$: In order to obtain an estimate of the form (2.28), we will use the results of [52], which generalise the $r^{p}$-weighted energy method of Dafermos and Rodnianski [20] to a more general setting of asymptotically flat backgrounds. Here, the asymptotic form of the metric (2.4) comes into play. Using the results of [52], the problem of proving (2.28) reduces to proving an integrated local energy decay statement of the form 


$$
\int_{0}^{\infty}\left\{\int_{\{t=\tau\} \cap\{r \leq R\}} J_{\mu}^{N}\left(\psi_{\leq \omega_{+}}\right) n^{\mu}\right\} d \tau \leq C(R) \cdot e^{C(R) \cdot \omega_{+}} \int_{\{t=0\}} J_{\mu}^{N}(\psi) n^{\mu} .
$$

Thus, the rest of the proof is centered around establishing the estimate (2.31).

Heuristically, the reason that (2.31) can be obtained lies in the fact that $\psi_{\leq \omega_{+}}$has bounded frequency support, and hence it does not experience trapping: Its energy will eventually "leak" (at a rate shrinking exponentially in $\omega_{+}$as $\omega_{+} \rightarrow \infty$ ) to $\mathcal{I}^{+}$or through $\mathcal{H}^{+}$. This phenomenon is captured by a Carleman-type inequality which is roughly of the form

$$
\begin{aligned}
& \int_{\mathcal{R}(0, T) \cap\left\{r_{1} \leq r \leq R\right\}} e^{s \cdot \omega_{+} w}\left\{\left|\psi_{\leq \omega_{+}}\right|^{2}+J_{\mu}^{N}\left(\psi_{\leq \omega_{+}}\right) n^{\mu}\right\} \\
& \leq C(R)\left(1+\left(s \omega_{+}\right)^{2}\right) \int_{\left.\mathcal{R}(0, T) \cap\left\{\frac{r_{1}}{2} \leq r \leq r_{1}\right\}\right)} e^{s \cdot \omega_{+} w}\left\{\left|\psi_{\leq \omega_{+}}\right|^{2}+J_{\mu}^{N}\left(\psi_{\leq \omega_{+}}\right) n^{\mu}\right\} \\
& \quad+C(R)\left(1+\left(s \omega_{+}\right)^{2}\right) \int_{\mathcal{R}(0, T) \cap\{R \leq r \leq R+1\})} e^{s \cdot \omega_{+} w}\left\{\left|\psi_{\leq \omega_{+}}\right|^{2}+J_{\mu}^{N}\left(\psi_{\leq \omega_{+}}\right) n^{\mu}\right\} \\
& \quad+C(R) \cdot e^{C(R) \cdot s \omega_{+}} \int_{\{t=0\}} J_{\mu}^{N}(\psi) n^{\mu} .
\end{aligned}
$$

In (2.32), $r_{1}>0$ should be considered small and $R>0$ large, $\mathcal{R}(0, T)$ denotes the region $\{0 \leq t \leq T\}$ for some arbitrary $T>0$, and $w$ is a suitable function on $\mathcal{D}$ which for the purpose of this discussion can be assumed to be a strictly increasing (but not bounded!) positive function of $r$. Finally, $s>0$ is a large constant.

The extraction of (2.32) follows closely the derivation of a similar Carleman type inequality in [58], using the multiplier method. The boundedness assumption 4 is used in an essential way to control boundary terms at $\{t=T\}$ appearing in this procedure.

Notice that due to the fact that $w$ has been chosen strictly increasing in $r$ (at least near the horizon and near null infinity), the boundary term near the horizon in the right hand side of (2.32) (namely the integral over $r \sim r_{1}$ ) carries a weight $e^{s \omega_{+} \cdot w}$ that is small in comparison to the weights $e^{s \omega_{+} \cdot w}$ in the bulk integral of the left hand side. The opposite happens with the boundary term near spacelike infinity $(r \sim R)$, where the $e^{s \omega_{+} w}$ weights should be considered large in comparison to the weights in the integral of the left hand side.

In order to attain the ILED statement (2.31), we would like to absorb the boundary terms of the right hand side of (2.32) by the left hand side, in a process producing error terms that can be bounded by the initial energy of $\psi$.

Treatment of the boundary terms near the horizon: In order to dispense with the first term of the right hand side of (2.32), one uses the positivity properties of the red shift vector field $N$ near the horizon (according to Assumption 2), as well as the fact that the weight $e^{s \omega_{+} w}$ in the $r \sim r_{1}$ region has much smaller values than in the $\left\{r \gg r_{1}\right\}$ region if $s$ is chosen sufficiently large. Let us remark at this point that Assumption 3 is also used here: The proof of the Carleman inequality (2.32) requires that the set $\left\{r_{1} \leq r \leq R\right\}$ does not intersect the ergoregion, while, in order to use Assumption 2 in order to deal with the first term of the right hand side of (2.32), it is necessary that 
$r_{0} \gtrsim r_{1}$ (where $r_{0}$ is the parameter appearing in Assumption 2). Thus, it is essential in this step that the ergoregion is contained in the set $\left\{r \lesssim r_{0}\right\}$, and this fact is guaranteed by Assumption 3.19

In particular, according to (2.8), the $K^{N}$ current is positive near the horizon and controls the first term of the right hand side of (2.32), while it becomes non positive only in the $r_{0} \leq r \leq 2 r_{0}$ region, where $r_{1}<r_{0}$. Hence, the first term in the right hand side of (2.32) can be bounded as:

$$
\begin{aligned}
& \int_{\left.\mathcal{R}(0, T) \cap\left\{\frac{r_{1}}{2} \leq r \leq r_{1}\right\}\right)}\left(1+\left(s \omega_{+}\right)^{2}\right) e^{s \cdot \omega_{+} w}\left\{\left|\psi_{\leq \omega_{+}}\right|^{2}+J_{\mu}^{N}\left(\psi_{\leq \omega_{+}}\right) n^{\mu}\right\} \\
& \leq C \cdot\left(1+\left(s \omega_{+}\right)^{2}\right)\left(\sup _{r \leq r_{1}} e^{s \omega_{+} w}\right) \int_{\left.\mathcal{R}(0, T) \cap\left\{r_{0} \leq r \leq 2 r_{0}\right\}\right)} \\
& \quad \times\left\{\left|\psi_{\leq \omega_{+}}\right|^{2}+J_{\mu}^{N}\left(\psi_{\leq \omega_{+}}\right) n^{\mu}\right\} \\
& \quad+C \cdot\left(1+\left(s \omega_{+}\right)^{2}\right)\left(\sup _{r \leq r_{1}} e^{s \omega_{+} w}\right) \cdot \int_{\{t=0\}} J_{\mu}^{N}(\psi) n^{\mu} .
\end{aligned}
$$

The second term of the right hand side of (2.33) is what we would like to end up with in the right hand side of (2.32), so we still have to dispense with the first term of (2.33). Notice that since $r_{1}<r_{0}$ and $w$ is a strictly increasing function of $r$, we can bound

$$
\sup _{r \leq r_{1}} e^{s \omega_{+} w} \leq e^{-c \cdot \omega_{+} s} \cdot \inf _{r_{0} \leq r \leq 2 r_{0}} e^{s \omega_{+} w}
$$

for some $c>0$ depending on the precise choice of $r_{1}, r_{0}$. Thus, if $s \gg 1$, we can etimate the first term of the right hand side of (2.33) by a small constant times the left hand side of (2.32) (recall $\left.\omega_{+} \geq 1\right)$ :

$$
\begin{aligned}
& \left(1+\left(s \omega_{+}\right)^{2}\right)\left(\sup _{r \leq r_{1}} e^{s \omega_{+} w}\right) \int_{\left.\mathcal{R}(0, T) \cap\left\{r_{0} \leq r \leq 2 r_{0}\right\}\right)}\left\{\left|\psi_{\leq \omega_{+}}\right|^{2}+J_{\mu}^{N}\left(\psi_{\leq \omega_{+}}\right) n^{\mu}\right\} \\
& \leq e^{-c \cdot \omega_{+} s} \cdot \int_{\mathcal{R}(0, T) \cap\left\{r_{1} \leq r \leq R\right\}} e^{s \cdot \omega_{+} w}\left\{\left|\psi_{\leq \omega_{+}}\right|^{2}+J_{\mu}^{N}\left(\psi_{\leq \omega_{+}}\right) n^{\mu}\right\} .
\end{aligned}
$$

Thus, the first term of the right hand side of (2.33) can be absorbed into the left hand side of (2.32) if $s$ is chosen sufficiently large in terms of the geometry of $(\mathcal{M}, g)$. Thus, (2.32), (2.33) and (2.35) yield:

$$
\begin{aligned}
& \int_{\mathcal{R}(0, T) \cap\{r \leq R\}} e^{s \cdot \omega_{+} w}\left\{\left|\psi_{\leq \omega_{+}}\right|^{2}+J_{\mu}^{N}\left(\psi_{\leq \omega_{+}}\right) n^{\mu}\right\} \\
& \leq C(R) \int_{\mathcal{R}(0, T) \cap\{R \leq r \leq R+1\})}\left(1+\left(s \omega_{+}\right)^{2}\right) e^{s \cdot \omega_{+} w}\left\{\left|\psi_{\leq \omega_{+}}\right|^{2}+J_{\mu}^{N}\left(\psi_{\leq \omega_{+}}\right) n^{\mu}\right\} \\
& \quad+C(R) \cdot e^{C(R) \cdot s \omega_{+}} \int_{\{t=0\}} J_{\mu}^{N}(\psi) n^{\mu}
\end{aligned}
$$

\footnotetext{
19 We should also note that in the case where $\mathcal{H}^{+}=\emptyset$, the first term of the right hand side of (2.32) can be dropped, and the integral in the left hand side of (2.32) is over the whole region $\mathcal{R}(0, T) \cap\{r \leq R\}$.
} 
Treatment of the boundary terms near infinity: Unfortunately, the previous trick does not apply in order to deal with the first term of the right hand side of (2.36), i.e. the $r \sim R$ boundary term: In this case, the weight $e^{s \omega_{+} w}$ is now much larger in the region $r \sim R$ than in the region $r<R$. Hence we can not hope to directly absorb the first term of the right hand side of (2.36) into the left hand side.

In order to deal with this term, therefore, a much more delicate analysis is necessary, and it is at this point that the results of [58] are used in a fundamental way. In particular, we will employ an ODE lemma proven in Sections 9 and 10 of [58], and we will apply it on a system of ordinary differential inequalities satisfied by the mass of $\omega$ and its derivatives on the cylinders $\{r=\rho\}$ (and varying eith $\rho$ ). This lemma will imply that, if $\omega$ solves the wave equation on $(\mathcal{D}, g)$ and has frequency support in $\left\{|\omega| \geq \omega_{1}\right\}$, then for any large $R_{f}$ and for any given $C_{2}>0$, for $R$ taking values in any interval of the form $\left[R_{f}, C \cdot R_{f}\right]$ the quantity $\int_{\mathcal{R}(0, T) \cap\{R \leq r \leq R+1\})}\left\{|\omega|^{2}+J_{\mu}^{N}(\omega) n^{\mu}\right\}$ will either decay as a function of $R$ with a $e^{-C_{2} \omega_{1} R}$ rate, or it will be bounded by $C\left(C_{2}\right)$ times the initial energy of $\omega$.

Assume, for a moment, that our solution $\psi_{\leq \omega_{+}}$actually has frequency support only in the regime $|\omega| \sim \omega_{+}$. Then the ODE lemma of Rodnianski and Tao would yield that, for a suitable choice of $C_{2} \gg 1$, the first term in the right hand side of (2.36) can either be absorbed by the left hand side, or can be bounded by the initial energy of $\psi$ (since, again, in our heuristic setting the energy of $\psi_{\leq \omega_{+}}$can be bounded by the energy of $\psi$ ). Thus, in the case where $\psi_{<\omega_{+}}$has frequency support only in the regime $|\omega| \sim \omega_{+},(2.36)$ readily implies the ILED statement:

$$
\int_{\mathcal{R}(0, T) \cap\{r \leq R\}} e^{s \cdot \omega_{+} w}\left\{\left|\psi_{\leq \omega_{+}}\right|^{2}+J_{\mu}^{N}\left(\psi_{\leq \omega_{+}}\right) n^{\mu}\right\} \leq C(R) \cdot e^{C(R) \cdot s \omega_{+}} \int_{\{t=0\}} J_{\mu}^{N}(\psi) n^{\mu} .
$$

In general, however, $\psi_{\leq \omega_{+}}$will not have frequency support only in the region $|\omega| \sim \omega_{+}$. For this reason, we need to decompose $\psi_{\leq \omega_{+}}$further into $\psi_{k}$ pieces, which have frequency supports in small intervals $\left[\omega_{k}, \omega_{k+1}\right]$ with comparable endpoints, except for $\psi_{0}$, which has frequency support in a small neighborhood around 0 . For each of the frequency decomposed components $\psi_{k}$, for $k \neq 0$, the previous heuristics work exactly as presented for $\psi_{\leq \omega_{+}}$, since the Carleman inequality (2.32) and the ODE lemma of Rodnianski and Tao hold for $\psi_{k}$ as well. Thus, after applying this procedure and then summing the $\psi_{k}$ 's for $k \neq 0$, this line of arguments establishes the integrated local energy decay statement (2.31) for $\psi_{\leq \omega_{+}}-\psi_{0}$ in place of $\psi_{\leq \omega_{+}}$.

Treatment of the very low frequency component $\psi_{0}$ : For $\psi_{0}$, a different argument needs to be furnished in order to reach the full integrated local energy decay statement (2.31). This is accomplished in Section 6, with the use of a special vector field current, coupled with a locally improved Morawetz ILED statement. At this point, Assumption 3 on the smallness of the ergoregion plays a crucial role.

\subsection{Remark on the Proof of Theorem 2.1}

We will only be concerned with proving Theorem 2.1 in the case where future horizon $\mathcal{H}^{+}$is non-empty. In the case $\mathcal{H}^{+}=\varnothing$, Assumption 2 can be completely dropped, 
and Assumption 3 degenerates to the condition that $g(T, T)<0$ everywhere on $\mathcal{D}$. Therefore, in the case $\mathcal{H}^{+}=\emptyset$, Theorem 2.1 can be established using almost the same arguments as in the case $\mathcal{H}^{+} \neq \emptyset$, and in fact the proof of the results of Sections 6 and 7 are simplified. Thus, from now on we will assume without loss of generality that $\mathcal{H}^{+} \neq \varnothing$ (the differences of the proof in the cases $\mathcal{H}^{+} \neq \varnothing$ and $\mathcal{H}^{+}=\emptyset$ will be highlighted in the footnotes of Sections 6 and 7).

In order to avoid confusion when doing estimates in each connected component of the asymptotically flat region $\mathcal{I}_{a s}$ of $\mathcal{D}$, we will assume without loss of generality that $\mathcal{I}_{a s}$ has only one connected component (i.e. that $(\mathcal{M}, g)$ has only one asymptotically flat end). This assumption will simplify the derivation of the estimates in the region $\{r \gg 1\}$ appearing in Sections 7-8, in view of the fact that $\{r \gg 1\}$ will be covered by a single polar coordinate chart. In the general case where $\mathcal{I}_{a s}$ has more than one components, the same estimates will follow after repeating the same proves on each component seperately and then adding the resulting estimates. The reader is advised to keep in mind this simplifying assumption when reading Sections 7-8.

It will also be convenient for the proof of Theorem 2.1 to assume without loss of generality that $\psi$ has compact initial data. This will not pose any further restriction for the statement of the theorem, since the full statement can then follow by a usual density argument. However, compact initial data for $\psi$ imply, due to the domain of dependence property, that the restriction of $\psi$ on any $\{t=$ const $\}$ hypersurface is also compactly supported, and in particular, $\psi$ must be supported in a set of the form $\left\{r \lesssim R_{\text {sup }}+|t|\right\}$ for some large $R_{\text {sup }}$ depending on $\psi .^{20}$

\subsection{Outline of the Paper}

The proof of Theorem 2.1 is presented in Sections 4-9. While reading these sections, one is advised to keep in mind the above heuristics, since despite their simplicity, the main arguments are often blurred by technicalities (mainly because of the need to carefully perform physical space as well as frequency space cut-offs, and also because of the very general assumptions on the geometry).

In Section 4, we carry out in detail the cut-off procedure in both physical and frequency space, and we establish all the required lemmas regarding the behaviour of $\psi_{\leq \omega_{+}}, \psi_{\geq \omega_{+}}$and $\psi_{k}$.

In Section 5, we obtain estimates for the behaviour of $\psi_{k}, \psi_{\leq \omega_{+}}, \psi_{\geq \omega_{+}}$in the asymtotically flat region $\{r \gg 1\}$. This is achieved by applying the new method of Dafermos and Rodnianski, originally appearing in [20], and generalised in [52] to include a broader class of asymptotically flat manifolds. In particular, in Section 5 the results of [52] are specialized for the case of the frequency decomposed components $\psi_{k}, \psi_{\leq \omega_{+}}, \psi_{\geq \omega_{+}}$of $\psi$.

In Section 6 , we provide the proof of integrated local energy decay for the very low frequency part $\psi_{0}$.

The proof of the integrated local energy decay of $\psi_{\leq \omega_{+}}$occupies Section 7 . There, we establish a Carleman type inequality for $\psi_{k}, k \neq 0$, which is then upgraded to an

$\overline{20}$ Of course no constant in the proof must be allowed to depend on $R_{\text {sup }}$ 
integrated local energy decay statement for $\psi_{\leq \omega_{+}}$with the use of a technical lemma of [58]. With this last statement at our disposal, and with the use of the generalised version of the $r^{p}$-weighted energy hierarchy of Dafermos and Rodnianski (from Section 4.2), we then infer in Section 8 that the local energy of $\psi_{\leq \omega_{+}}$decays polynomially in time.

The proof of Theorem 2.1 is completed in Section 9, where the interpolation scheme (2.29) is rigorously formulated.

The proof of Corollary 2.2 is presented in Section 10, while the proof of Corollaries 2.3 and 2.4 are presented in Section 11. Finally, we show that the result of Theorem 2.1 is optimal in this generality, by establishing the sharpness of the logarithmic decay rate in Section 12.

\section{Notational Conventions and Hardy Inequalities}

In this paper, we will adopt the same notational conventions as we did in [52]. We will now proceed to describe in more detail these conventions.

\subsection{Constants and Parameters}

We will adopt the following convention for denoting constants appearing in inequalities, as is done in [21]: Capital letters (e.g. C) will be used to denote "large" constants, typically appearing on the right hand side of inequalities. (Such constants can be "freely" replaced by larger ones without rendering the inequality invalid.) We will use lower case letters (e.g. c) to denote "small" constants (which can similarly freely be replaced by smaller ones). The same characters will be frequently used to denote different constants.

We will assume that all non-explicit constants will depend on the specific geometric aspects of our spacetime (e.g the topology and the exact form of the metric) and we will not keep track of this dependence, except for some very specific cases. However, since we will need to define a plethora of parameters in the following sections, we will always keep track of the dependence of all constants on each of these parameters. Once a parameter is fixed (which will be clearly stated in the text), the dependence of constants on it will be dropped.

The parameter $R_{1}$, appearing in the statement of Theorem 2.1, will be considered fixed, and hence we will drop any explicit referrence to it from constants depending on its choice, unless there is a need to emphasize this dependence. Since we can always increase $R_{1}$ without affecting the statement of Theorem 2.1, we will assume without loss of generality that $R_{1}$ is large enough so that the region $\left\{r \geq R_{1}\right\}$ is contained in the chart where the metric $g$ takes the form (2.4).

\subsection{Inequality Symbols}

We will use the notation $f_{1} \lesssim f_{2}$ for two real functions $f_{1}, f_{2}$ as usual to imply that there exists some $C>0$, such that $f_{1} \leq C \cdot f_{2}$. This constant $C$ might depend on free parameters, and these parameters will be stated clearly in each case. If nothing is 
stated regarding the dependence of this constant on parameters, it should be assumed that it only depends on the geometry of $(\mathcal{D}, g)$, and on the parameter $R_{1}$ appearing in the statement of Theorem 2.1.

We will write $f_{1} \sim f_{2}$ when we can bound $f_{1} \lesssim f_{2}$ and $f_{2} \lesssim f_{1}$. The notation $f_{1} \ll f_{2}$ will be equivalent to the statement that $\frac{\left|f_{1}\right|}{\left|f_{2}\right|}$ can be bounded by some sufficiently small positive constant, the magnitude and the dependence of which on variable parameters will be clear in each case from the context. For any function $f: \mathcal{M} \rightarrow[0,+\infty),\{f \gg 1\}$ will denote the subset $\{f \geq C\}$ of $\mathcal{M}$ for some constant $C \gg 1$.

For functions $f_{1}, f_{2}:\left[x_{0},+\infty\right) \rightarrow \mathbb{R}$, the notation $f_{1}=o\left(f_{2}\right)$ will mean that $\frac{\left|f_{1}\right|}{\left|f_{2}\right|}$ can be bounded by some continuous function $h:\left[x_{0},+\infty\right) \rightarrow(0,+\infty)$ such that $h(x) \rightarrow 0$ as $x \rightarrow+\infty$. This bound $h$ might deppend on free parameters, and this fact will be clear in each case from the context.

\subsection{Coordinate Charts on $\mathcal{D}$ and Subsets of $\mathcal{D}$ Associated to $t$}

In this paper, we will identify $\mathcal{D} \backslash \mathcal{H}^{-}$with $\mathbb{R} \times(\Sigma \cap \mathcal{D})$ by setting $\{t\} \times(\Sigma \cap \mathcal{D})$ to be equal to the image of $\Sigma \cap \mathcal{D}$ under the flow of the vector field $T$ for time $t$ (see the remarks in Assumption 1.

When performing calculations in specific coordinate charts on regions of $\mathcal{D} \backslash \mathcal{H}^{-}$, we will always pick coordinate charts of the following form: For any local coordinate chart $x=\left(x^{1}, x^{2}, \ldots, x^{d}\right)$ on an open subset $\mathcal{V}$ of $\Sigma \cap \mathcal{D}$, we will extend the functions $x^{i}$ on the whole of $\mathbb{R} \times \mathcal{V} \subset \mathcal{D} \backslash \mathcal{H}^{-}$by the requirement $T\left(x^{i}\right)=0$, and we will use the coordinate chart $\left(t, x^{1}, \ldots, x^{d}\right)$ on $\mathbb{R} \times \mathcal{V}$. Notice that in such a coordinate chart we have $\partial_{t} \equiv T$. In the expression of any tensor $\mathfrak{w}$ in such a coordinate chart, components with indices ranging from 1 to $d$ will correspond to the components of the tensor associated to $\partial_{x^{1}}, \ldots, \partial_{x^{d}}$ (or $d x^{1}, \ldots, d x^{d}$ ) respectively.

For any $t_{1} \leq t_{2}$, we will denote

$$
\mathcal{R}\left(t_{1}, t_{2}\right) \doteq\left\{t_{1} \leq t \leq t_{2}\right\} \subset \mathcal{D} .
$$

Furthermore, for any $t_{0} \in \mathbb{R}$, we will denote:

$$
\Sigma_{t_{0}} \doteq\left\{t=t_{0}\right\}
$$

\subsection{Connections and Volume Forms}

We will usually denote the natural connection of a pseudo-Riemannian manifold $\left(\mathcal{N}, h_{\mathcal{N}}\right)$ as $\nabla^{h_{\mathcal{N}}}$ or $\nabla_{h_{\mathcal{N}}}$ (or simply $\nabla_{\mathcal{N}}$ when there is no ambiguity about the metric $h_{\mathcal{N}}$ ). The associated volume form will be denoted as $d h_{\mathcal{N}}$. If $h_{\mathcal{N}}$ is Riemannian, $|\cdot|_{h_{\mathcal{N}}}$ will denote the associated norm on the tensor bundle of $\mathcal{N}$.

For any integer $l \geq 0,\left(\nabla^{h}\right)^{l}$ or $\nabla_{h_{\mathcal{N}}}^{l}$ will denote the higher order operator

$$
\underbrace{\nabla_{h_{\mathcal{N}}} \cdots \nabla_{h_{\mathcal{N}}}}_{l \text { times }} .
$$


We should remark that the product (3.3) is not symmetrised. We will also adopt the convention that we will always use Latin characters to denote such powers of covariant derivative operators. On the other hand, Greek characters will be used for the indices of a tensor in an abstract index notation.

Let us state an example of the above convention: Let $k$ be a $\left(n_{1}, n_{2}\right)$-tensor and $\omega:\left(\mathcal{N}, h_{\mathcal{N}}\right) \rightarrow \mathbb{C}$ be a smooth function. Then the quantity

$$
k_{\beta_{1} \ldots \beta_{n_{2}}}^{\alpha_{1} \ldots \alpha_{n_{1}}} \cdot\left(\nabla_{h_{\mathcal{N}}}^{n_{1}+n_{2}}\right)_{\alpha_{1} \ldots \alpha_{n_{1}}}^{\beta_{1} \ldots \beta_{n_{2}}} \varphi
$$

denotes the contraction of the $n_{1}+n_{2}$ order derivative $\nabla_{h_{\mathcal{N}}}^{n_{1}+n_{2}} \omega$ of $\omega$ with the tensor $k$. In the above, the metric $h_{\mathcal{N}}$ was used to raise the first $n_{2}$ indices of $\nabla_{h_{\mathcal{N}}}^{n_{1}+n_{2}} \omega$. Notice that in (3.4) we have used the abstract index notation, and hence the indices in (3.4) are not associated to any fixed local coordinate chart.

\subsection{Integration over Domains and Hypersurfaces}

In the cases where we use the natural volume form $\omega$ associated to the metric $g$ of a Lorentzian manifold $(\mathcal{M}, g)$ in order to integrate over open subsets of $\mathcal{M}$, the volume form will be often dropped in the expression for the integral. Recall that in any local coordinate chart $\left(x^{0}, x^{1}, x^{2}, \ldots x^{d}\right), \omega$ is expressed as

$$
\omega=\sqrt{-\operatorname{det}(g)} d x^{0} \cdots d x^{d} .
$$

We will apply the same rule when integrating over any spacelike hypersurface $\mathcal{S}$ of $(\mathcal{M}, g)$ using the natural volume form of its induced (Riemannian) metric.

In the case of a smooth null hypersurface $\mathscr{H}$, the volume form with which integration will be considered will as usual depend on the choice of a future directed null generator $n_{\mathscr{H}}$ for $\mathscr{H}$. For any such choice of $n_{\mathscr{H}}$, selecting an arbitrary vecor field $X$ on $T_{\mathscr{H}} M$ such that $g\left(X, n_{\mathscr{H}}\right)=-1$ enables the construction of a non degenerate $d$-form on $\mathscr{H}: d \operatorname{vol}_{n_{\mathscr{H}}} \doteq i_{X} \omega$, which depends on the on the precise choice of $n_{\mathscr{H}}$, but not on the choice for $X$. In that case, $d v o l_{n_{\mathscr{H}}}$ will be the volume form on $\mathscr{H}$ associated with $n_{\mathscr{H}}$.

\subsection{Notations for Derivatives on $\mathbb{S}^{d-1}$}

Since, in the present paper, we will frequently work in polar coordinates in the asymptotically flat region of $(\mathcal{D}, g)$, we will introduce some convenient shorthand notation regarding iterated derivatives on the unit sphere $\mathbb{S}^{d-1}, d \geq 3$.

The usual round metric on the sphere $\mathbb{S}^{d-1}$ will be denoted as $g_{\mathbb{S}^{d-1}}$. This is simply the induced metric on the unit sphere of $\mathbb{R}^{d}$. We will also denote with $g_{\mathbb{S}^{d-1}}$ the natural extension of the round metric to an inner product on the space of tensors over $\mathbb{S}^{d-1}$. According to the conventions of Section 3.4, for any tensor field $\mathfrak{w}$ on $\mathbb{S}^{d-1}$, $|\mathfrak{w}|_{g_{\mathbb{S}} d-1}$ will denote the norm of $\mathfrak{w}$ with respect to $g_{\mathbb{S}^{d-1}}$ and $\nabla^{\mathbb{S}^{d-1}}$ (or $\nabla_{\mathbb{S} d-1}$ ) will denote the covariant derivative associated with $g_{\mathbb{S}^{d-1}}$. Furthermore, for any smooth 
$\left(n_{1}, n_{2}\right)$-tensor field $\mathfrak{w}$ on $\mathbb{S}^{d-1},\left(\nabla^{\mathbb{S}^{d-1}}\right)^{k} \mathfrak{w}\left(\right.$ or $\left.\nabla_{\mathbb{S} d-1}^{k} \mathfrak{w}\right)$ will denote the $\left(n_{1}, n_{2}+k\right)$ tensor field on $\mathbb{S}^{d-1}$ obtained after applying the operator $\nabla^{\mathbb{S}^{d-1}}$ on $\mathfrak{w} k$ times. The Laplace-Beltrami operator on $\left(\mathbb{S}^{d-1}, g_{\mathbb{S}^{d-1}}\right)$ will be denoted as $\Delta_{g_{\mathbb{S}} d-1}$.

Frequently, we will work on regions $\mathcal{U}$ of a spacetime $\mathcal{M}^{d+1}$ such that $\mathcal{U}$ can be mapped diffeomorphically, through a coordinate "chart", onto $\mathbb{R}_{+} \times \mathbb{R}_{+} \times \mathbb{S}^{d-1}$. In any such a coordinate "chart", $\sigma$ will denote the projection $\sigma: \mathcal{U} \rightarrow \mathbb{S}^{d-1}$. We remark that, for any $x \in \mathcal{M}, \sigma(x)$ is a point on $\mathbb{S}^{d-1}$ and not just the coordinates of this point in a coordinate chart on $\mathbb{S}^{d-1}$. The same $\sigma$ notation will also be used for the spherical variable of a polar coordinate "chart" on codimension 1 submanifolds of $\mathcal{M}$ (in this case, the range of such a "chart" will be simply $\mathbb{R}_{+} \times \mathbb{S}^{d-1}$ ). For example, we will use the notation $(r, \sigma):\left\{x^{0}=0\right\} \rightarrow \mathbb{R}_{+} \times \mathbb{S}^{d-1}$ for the usual polar coordinate chart on the hyperplane $\left\{x^{0}=0\right\}$ of $\mathbb{R}^{d+1}$.

On a subset $\mathcal{U}$ of a spacetime $\mathcal{M}$ covered by a polar coordinate chart $\left(u_{1}, u_{2}, \sigma\right)$ : $\mathcal{U} \rightarrow \mathbb{R}_{+} \times \mathbb{R}_{+} \times \mathbb{S}^{d-1}$, for any function $h: \mathcal{U} \rightarrow \mathbb{C}$ and any $\beta_{1}, \beta_{2} \in \mathbb{R}_{+}, h\left(\beta_{1}, \beta_{2}, \cdot\right)$ defines a function on $\mathbb{S}^{d-1}$. Under this correspondence, the $\nabla^{\mathbb{S}^{d-1}}$ differential operator on $\mathbb{S}^{d-1}$ is extended to a tangential differential operator on the hypersurfaces $\left\{u_{1}, u_{2}=\right.$ const $\} \subset \mathcal{U}$. This operator is, of course, related to the specific choice of the polar coordinate chart $\left(u_{1}, u_{2}, \sigma\right)$.

The following schematic notation for derivatives on $\mathbb{S}^{d-1}$ (and the associated tangential operators on the hypersurfaces $\left\{u_{1}, u_{2}=\right.$ const $\}$ in a $\left(u_{1}, u_{2}, \sigma\right)$ coordinate chart on a spacetime $\mathcal{M}$ ) will be frequently used in the present paper: For any function $h: \mathbb{S}^{d-1} \rightarrow \mathbb{C}$ and any $l \in \mathbb{N}$, we will denote the $l$-th order derivative $\nabla_{\mathbb{S}^{d-1}}^{l} h$ as $\partial_{\sigma}^{l} h$. The norm of this tensor will be denoted as:

$$
\left|\partial_{\sigma}^{l} h\right| \doteq\left|\nabla_{\mathbb{S}^{d-1}}^{l} h\right|_{\mathbb{S}^{d-1}}
$$

Furthermore, for any symmetric $(l, 0)$-tensor $b$ on $\mathbb{S}^{d-1}$, the following schematic notation for the contraction of $\left(\nabla^{\mathbb{S}^{d-1}}\right)^{l} h$ with $b$ will be frequently used:

$$
b \cdot \partial_{\sigma}^{l} h \doteq b^{\iota_{1} \ldots \iota_{l}}\left(\nabla_{\mathbb{S}^{d-1}}^{l}\right)_{\iota_{1} \ldots \iota_{l}} h
$$

(for the notations on powers of covariant derivatives and the abstract index notation, see Section 3.4). The same notation will be used for the contraction of the product of derivatives of multiple functions: For any family of $m$ functions $h_{1}, \ldots, h_{m}: \mathbb{S}^{d-1} \rightarrow$ $\mathbb{C}$ and any set $\left(j_{1}, \ldots j_{m}\right)$ of non-negative integers, for any $\left(\sum_{k=1}^{n} j_{k}, 0\right)$-tensor $b$ on $\mathbb{S}^{d-1}$ which is symmetric in any pair of indices lying in the same one of the intervals $I_{n}=\left(\sum_{k=1}^{n-1} j_{k}+1, \sum_{k=1}^{n} j_{k}\right)$ for each $n \in\{1, \ldots m\}$, we will adopt the notation

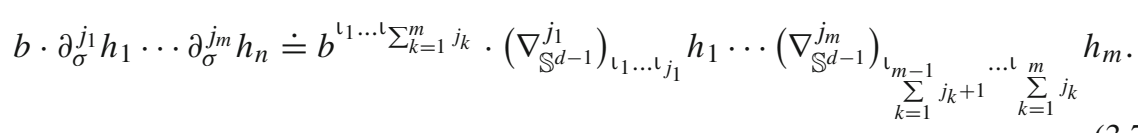

Notice that the tensor $b$ used in the notation (3.7) will not necessarily be symmetric in pairs of indices lying in seperate pairs of the $I_{n}$ intervals. 
We will also use the same notation (i.e. (3.6) and (3.7)) when $h, h_{1}, \ldots, h_{m}$ are tensor fields on $\mathbb{S}^{d-1}$.

Notice also that, working in a polar coordinate "chart" $\left(u_{1}, u_{2}, \sigma\right): \mathcal{U} \rightarrow \mathbb{R}_{+} \times$ $\mathbb{R}_{+} \times \mathbb{S}^{d-1}$, the following commutation relation holds for any function $h$ on $\mathcal{U}$ :

$$
\left[\mathcal{L}_{\partial_{u_{i}}} \nabla^{\mathbb{S}^{d-1}}, \nabla^{\mathbb{S}^{d-1}} \partial_{u_{i}}\right] h=0
$$

where $\partial_{u_{i}}$ is the coordinate vector field associated to the coordinate function $u_{i}$ (for $i=1,2)$. Therefore, we will frequently denote

$$
\mathcal{L}_{\partial_{u_{i}}} \nabla^{\mathbb{S}^{d-1}} h \doteq \partial_{u_{i}} \partial_{\sigma} h
$$

and this notation will allow commuting $\partial_{u_{i}}$ with $\partial_{\sigma}$, as if $\partial_{\sigma}$ was a regular coordinate vector field.

The notation $d \sigma$ will be used in two different ways, depending on the context: it will denote either the usual volume form on $\left(\mathbb{S}^{d-1}, g_{\mathbb{S} d-1}\right)$ or a 1 -form on $\mathbb{S}^{d-1}$ satisfying for $0 \leq k \leq 4$ the bound $\left|\left(\nabla^{\mathbb{S}^{d-1}}\right)^{k} d \sigma\right|_{g_{\mathbb{S} d-1}} \leq 1$. Similarly, $d \sigma d \sigma$ will denote a symmetric $(2,0)$-tensor on $\mathbb{S}^{d-1}$ satisfying for any $0 \leq k \leq 4$ the bound $\left|\left(\nabla^{\mathbb{S}^{d-1}}\right)^{k}(d \sigma d \sigma)\right|_{g_{\mathbb{S}^{d-1}}} \leq 1$.

Example For any function $f$ and any tensor $b$ on $\mathbb{S}^{d-1}$ with the aforementioned symmetries, the above notation will allow us to perform the following integration by parts procedure:

$$
\int_{\mathbb{S} d-1} b \cdot \partial_{\sigma} f \cdot \partial_{\sigma} \partial_{\sigma} f d \sigma=-\frac{1}{2} \int_{\mathbb{S} d-1}\left(c_{1} \partial_{\sigma} b+c_{2} b\right) \cdot \partial_{\sigma} f \cdot \partial_{\sigma} f d \sigma
$$

for some smooth contracting tensors $c_{1}, c_{2}$ which are bounded with bounds depending only on the tensor type of $b$.

The notation (3.6) and (3.7) will be frequently used in cases where we lack an explicit form for the contracting tensor $b$, but we have bounds for the norm of $b$ and its derivatives. This is the reason motivating our choice of a notation which apparently loses information regarding the structure of the underlying expression.

\subsection{The $O_{k}(\cdot)$ Notation}

For any integer $k \geq 0$ and any $b \in \mathbb{R}$, the notation $h=O_{k}\left(r^{b}\right)$ for some smooth function $h: \mathcal{M} \rightarrow \mathbb{C}$ will be used to denote that in the $(t, r, \sigma)$ polar coordinate chart in the region $\{r \gg 1\}$ of $\mathcal{D}$ (see Assumption 1):

$$
\sum_{j=0}^{k} \sum_{j_{1}+j_{2}+j_{3}=j} r^{j_{1}+j_{2}}\left|\partial_{t}^{j_{1}} \partial_{r}^{j_{2}} \partial_{\sigma}^{j_{3}} h\right| \leq C \cdot r^{b}
$$


for some constant $C>0$ dependng on $k$ and $h$. The same notation (omitting the $\partial_{t}$ derivatives) will also be used for functions on regions of manifolds cover by an $(r, \sigma)$ polar coordinate chart.

\subsection{Vector Field Multipliers and Currents}

In the present paper, we will frequently use the language of currents and vector field multipliers in order to establish the desired estimates. On any Lorentzian manifold $(\mathcal{M}, g)$, associated to the wave operator $\square_{g}=\frac{1}{\sqrt{-\operatorname{det}(g)}} \partial_{\mu}\left(\sqrt{-\operatorname{det}(g)} \cdot g^{\mu \nu} \partial_{\nu}\right)$ is a $(0,2)$-tensor called the energy momentum tensor $T$. For any smooth function $\psi$ : $\mathcal{M} \rightarrow \mathbb{C}$, the energy momentum tensor takes the form

$$
T_{\mu \nu}(\psi)=\frac{1}{2}\left(\partial_{\mu} \psi \cdot \partial_{\nu} \bar{\psi}+\partial_{\mu} \bar{\psi} \cdot \partial_{\nu} \psi\right)-\frac{1}{2}\left(\partial^{\lambda} \psi \cdot \partial_{\lambda} \bar{\psi}\right) g_{\mu \nu}
$$

For any continuous and piecewise $C^{1}$ vector field $X$ on $\mathcal{M}$, the following associated currents can be defined almost everywhere:

$$
\begin{aligned}
J_{\mu}^{X}(\psi) & =T_{\mu \nu}(\psi) X^{\mu}, \\
K^{X}(\psi) & =T_{\mu \nu}(\psi) \nabla^{\mu} X^{\nu} .
\end{aligned}
$$

The following divergence identity then holds almost everywhere:

$$
\nabla^{\mu} J_{\mu}^{X}(\psi)=K^{X}(\psi)+\operatorname{Re}\left\{\left(\square_{g} \psi\right) \cdot X \bar{\psi}\right\}
$$

\subsection{Hardy-Type Inequalities}

Frequently throughout this paper, we will need to control the weighted $L^{2}$ norm of some function $u$ by some weighted $L^{2}$ norm of its derivative $\nabla u$. This will always be accomplished with the use of some variant of the following Hardy-type inequality on $\mathbb{R}^{d}$ (which is true for $d \geq 1$, although we will only need it for $d \geq 3$ ):

Lemma 3.1 For any $a>0$, there exists some $C_{a}>0$ such that for any smooth and compactly supported function $u: \mathbb{R}^{d} \rightarrow \mathbb{C}$ and any $R>0$ we can bound

$$
\begin{aligned}
& \int_{\mathbb{R}^{d} \cap\{r \geq R\}} r^{-d+a} \cdot|u|^{2} d x+\int_{\{r=R\}} R^{-(d-1)+a} \cdot|u|^{2} d g_{\{r=R\}} \\
& \quad \leq C_{a} \int_{\mathbb{R}^{d} \cap\{r \geq R\}} r^{-(d-2)+a}\left|\partial_{r} u\right|^{2} d x
\end{aligned}
$$

In the above, $r$ is the polar distance on $\mathbb{R}^{d}, d x$ is the usual volume form on $\mathbb{R}^{d}$ and $d g_{\{r=R\}}$ is the volume form of the induced metric on the sphere $\{r=R\} \subset \mathbb{R}^{d}$.

Proof Following the usual steps for proving a Hardy inequality, we can calculate in polar coordinates: 


$$
\begin{aligned}
\int_{\mathbb{R}^{d} \cap\{r \geq R\}} r^{-d+a} \cdot|u|^{2} d x= & \int_{\mathbb{S}^{d-1}}\left(\int_{R}^{\infty} r^{-d+a} \cdot|u|^{2} r^{d-1} d r\right) d g_{\mathbb{S}^{d-1}} \\
= & \int_{\mathbb{S}^{d-1}}\left(\int_{R}^{\infty} r^{-1+a} \cdot|u|^{2} d r\right) d g_{\mathbb{S}^{d-1}} \\
= & \frac{1}{a} \int_{\mathbb{S}^{d-1}}\left(\int_{R}^{\infty} \partial_{r}\left(r^{a}\right) \cdot|u|^{2} d r\right) d g_{\mathbb{S}^{d-1}} \\
= & \frac{1}{a}\left\{-\left.\int_{\mathbb{S}^{d-1}}\left(r^{a} \cdot|u|^{2}\right)\right|_{r=R} d g_{\mathbb{S}^{d-1}}\right. \\
& \left.+2 \int_{\mathbb{S}^{d-1}}\left(\int_{R}^{\infty} r^{a} \cdot \operatorname{Re}\left(\partial_{r} u \cdot \bar{u}\right) d r\right) d g_{\mathbb{S}^{d-1}}\right\},
\end{aligned}
$$

where, in order to integrate by parts, we have used the fact that $u$ was compactly supported.

Moving the first term of the right hand side of (3.17) to the left hand side, and using the fact that in polar coordinates $d g_{\{r=R\}}=R^{d-1} d g_{\mathbb{S}^{d-1}}$ and $d x=r^{d-1} d r d g_{\mathbb{S}^{d-1}}$, we obtain:

$$
\begin{aligned}
& \int_{\mathbb{R}^{d} \cap\{r \geq R\}} r^{-d+a} \cdot|u|^{2} d x+\frac{1}{a} \int_{\{r=R\}} R^{-(d-1)+a} \cdot|u|^{2} d g_{\{r=R\}} \\
& =\frac{2}{a} \int_{\mathbb{R}^{d} \cap\{r \geq R\}} r^{-(d-1)+a} \cdot \operatorname{Re}\left(\partial_{r} u \cdot \bar{u}\right) d x \\
& \leq \frac{2}{a}\left(\int_{\mathbb{R}^{d} \cap\{r \geq R\}} r^{-(d-2)+a} \cdot\left|\partial_{r} u\right|^{2} d x\right)^{1 / 2} \\
& \quad \times\left(\int_{\mathbb{R}^{d} \cap\{r \geq R\}} r^{-d+a} \cdot|u|^{2} d x\right)^{1 / 2} .
\end{aligned}
$$

The desired inequality (3.16) now readily follows after absorbing the second factor of the right hand side of (3.18) into the left hand side.

\section{Construction of the Frequency Decomposed Components of $\psi$}

In this section, we will assume that we are given a smooth function $\psi: \mathcal{D} \rightarrow \mathbb{C}$ as in the statement of Theorem 2.1 solving $\square_{g} \psi=0$ on $J^{+}(\Sigma) \cap \mathcal{D}$ with compactly supported initial data on $\Sigma$. We will introduce the parameters $t^{*}>0, \omega_{+}>1$ and $0<\omega_{0}<1$, and we will decompose the function $\psi$ into components with localised frequency support in the $t$ variable. We will always identify $\mathcal{D} \backslash \mathcal{H}^{-}$with $\mathbb{R} \times \Sigma_{0}=\mathbb{R} \times(\Sigma \cap \mathcal{D})$ under the flow of $T$ as explained in Section 3.3.

In order to be able to apply the Fourier transform in the time variable, we will first need to multiply $\psi$ with a suitable cut-off function in time, so that the resulting function has compact support in $t$. This cut off procedure will be similar to the one followed by Dafermos-Rodnianski in [21]. We will then establish various estimates for the frequency decomposed components of (the cut-off of) $\psi$. We will now proceed with the details. 


\subsection{Frequency Cut-Off}

Let $\chi_{1}: \mathbb{R}_{+} \rightarrow[0,1]$ be a smooth function such that $\chi_{1} \equiv 0$ on $x \leq R_{1}$ and $\chi_{1} \equiv 1$ on $x \geq R_{1}+1$, for the given $R_{1}$ in the statement of Theorem 2.1. Since we can always increase $R_{1}$ without affecting the statement of Theorem 2.1, whenever needed we will assume without loss of generality that $R_{1}$ is large enough in terms of the geometry of $(\mathcal{D}, g)$.

We define the following "distorted" time functions on $\mathcal{D}$ :

$$
\begin{gathered}
t_{+}=t-\frac{1}{2} \chi_{1}(r)\left(r-R_{1}\right), \\
t_{-}=t+\frac{1}{2} \chi_{1}(r)\left(r-R_{1}\right) .
\end{gathered}
$$

Note that the level sets of these time functions are indeed spacelike hypersurfaces (provided $R_{1} \gg 1$ ), ${ }^{21}$ and they agree with the level sets of $t$ on $\left\{r \leq R_{1}\right\}$. Moreover:

$$
\left\{t_{+}=0\right\} \subset J^{+}(\{t=0\}) \subset J^{+}\left(\left\{t_{-}=0\right\}\right) .
$$

The reason for introducing these distorted time functions will become apparent in the next section, when we will need to exchange decay in time with decay in $r$.

We will now describe the cut off of procedure in the time variable: Let $\chi_{2}: \mathbb{R} \rightarrow$ $[0,1]$ be a smooth function such that $\chi_{2} \equiv 1$ on $[1,+\infty)$ and $\chi_{2} \equiv 0$ on $(-\infty, 0]$. Then for any given $t^{*}>0$, we define the smooth cut-off function $h_{t^{*}}: \mathcal{D} \rightarrow[0,1]$ :

$$
h_{t^{*}}=h_{t^{*}}(t, r)=\chi_{2}\left(t_{-}(t, r)\right) \chi_{2}\left(t^{*}-t_{+}(t, r)\right) .
$$

We observe that $\operatorname{supp}\left(h_{t^{*}}\right) \subseteq\left\{t^{+} \leq t^{*}\right\} \cap\left\{t^{-} \geq 0\right\}$ and $\operatorname{supp}\left(\nabla h_{t^{*}}\right) \subseteq\left\{0 \leq t_{-} \leq\right.$ $1\} \cup\left\{t^{*}-1 \leq t_{+} \leq t^{*}\right\}$. It is also readily verified that $\sup \left|\nabla h_{t^{*}}\right|,\left|\nabla^{2} h_{t^{*}}\right| \leq C$ for a constant $C$ independent of $t^{*}$.

Given a positive real number $t^{*}$ as before, we will define the time cut-off $\psi_{t^{*}}$ of $\psi$ as

$$
\psi_{t^{*}}=h_{t^{*}} \cdot \psi .
$$

Observe that $\psi_{t^{*}}$ satisfies the equation

$$
\square_{g} \psi_{t^{*}}=F,
$$

where

$$
F=\partial^{\mu} h_{t^{*}} \cdot \partial_{\mu} \psi+\left(\square_{g} h_{t^{*}}\right) \cdot \psi
$$

is supported in $\left\{0 \leq t_{-} \leq 1\right\} \cup\left\{t^{*}-1 \leq t_{+} \leq t^{*}\right\}$.

In view of the assumption that the initial data for $\psi$ on $\{t=0\}$ are supported in a set of the form $\left\{r \leq R_{\text {sup }}\right\}$ (see Section 2.6), we infer that $\psi_{t^{*}}$ is supported in a

21 Terminating at spacelike infinity. 
cylinder of the form $\left\{r \lesssim R_{\text {sup }}+t^{*}\right\}$. This fact will serve to show that some spacetime integrals of $\psi_{t^{*}}$ are well defined at various points throughout the proof.

Note that for any $\tau \in\left[0, t^{*}\right]$ :

$$
\begin{aligned}
\int_{t=\tau} J_{\mu}^{N}\left(\psi_{t^{*}}\right) n^{\mu} & \leq C \cdot \int_{t=\tau}\left(h_{t^{*}} J_{\mu}^{N}(\psi) n^{\mu}+\left|\nabla h_{t^{*}}\right| \cdot|\psi|^{2}\right) \\
& \leq C \cdot\left(\int_{t=\tau} h_{t^{*}} J_{\mu}^{N}(\psi) n^{\mu}+\int_{\{t=\tau\} \cap\left\{r \leq 2 R_{1}\right\}}|\psi|^{2}\right) \\
& \leq C \cdot \int_{t=\tau} J_{\mu}^{N}(\psi) n^{\mu} \leq C \cdot \int_{t=0} J_{\mu}^{N}(\psi) n^{\mu} .
\end{aligned}
$$

We will also need to perform a cut-off procedure in the frequency domain: Let $\omega_{0}>0$ be a (small) positive constant, and $\omega_{+} \gg \omega_{0}$ a (large) positive constant. We decompose the interval $\left[\omega_{0}, \omega_{+}\right]$into a finite number of closed intervals $\left\{\left[\omega_{k}, \omega_{k+1}\right]\right\}_{k=0}^{n-1}$ such that $\omega_{n}=\omega_{+}$and $\frac{1}{4} \omega_{0}<\omega_{k+1}-\omega_{k}<\frac{1}{2} \omega_{0}$. Note that $n \sim \frac{\omega_{+}}{\omega_{0}}$. We will also set for $-n \leq k \leq-1$ : $\omega_{k}=-\omega_{-k}$.

Fix a smooth function $\chi_{3}: \mathbb{R} \rightarrow[0,1]$ such that $\chi_{3} \equiv 1$ on $[-1,1]$ and $\chi_{3} \equiv 0$ outside $\left(-\frac{9}{8}, \frac{9}{8}\right)$, and define the smooth cut-off function $\zeta_{\leq \omega_{+}}: \mathbb{R} \rightarrow[0,1]$,

$$
\zeta_{\leq \omega_{+}}(\omega)=\chi_{3}\left(\frac{\omega}{\omega_{+}}\right) .
$$

We also set $\zeta_{\geq \omega_{+}}=1-\zeta_{\leq \omega_{+}}$. These two functions will be used to split $\psi_{t^{*}}$ into a high frequency component $\psi_{\geq \omega_{+}}$and a low frequency component $\psi_{\leq \omega_{+}}$.

As we remarked in Section 2.5, we will need to perform a finer frequency cut-off on the low frequency component $\psi_{\leq \omega_{+}}$. To this end, we also define the following cut-off functions on $\mathbb{R}$ :

$$
\tilde{\zeta}_{0}(\omega)=\chi_{3}\left(\frac{\omega}{\omega_{0}}\right)
$$

and for $1 \leq k \leq n$ :

$$
\tilde{\zeta}_{k}(\omega)=\chi_{3}\left(\frac{\omega-\frac{\omega_{k-1}+\omega_{k}}{2}}{\frac{1}{2}\left(\omega_{k}-\omega_{k-1}\right)}\right) .
$$

For $-n \leq k \leq-1$, we set $\tilde{\zeta}_{k}(\omega) \doteq \tilde{\zeta}_{-k}(-\omega)$. Finally, for $-n \leq k \leq n$, we define the functions

$$
\zeta_{k}(\omega)=\frac{\tilde{\zeta}_{k}(\omega)}{\sum_{i=-n}^{n} \tilde{\zeta}_{i}(\omega)} .
$$

The properties of the functions $\zeta_{k}$ that we will need are the following:

- $\operatorname{supp}\left(\zeta_{\leq \omega_{+}}\right) \subseteq\left[-\frac{9}{8} \omega_{+}, \frac{9}{8} \omega_{+}\right], \operatorname{supp}\left(\zeta_{\geq \omega_{+}}\right) \subseteq\left(-\infty,-\omega_{+}\right] \cup\left[\omega_{+},+\infty\right)$

- $\operatorname{supp}\left(\zeta_{0}\right) \subseteq\left[-\frac{9}{8} \omega_{0}, \frac{9}{8} \omega_{0}\right]$

- For $1 \leq k \leq n$ :

$\operatorname{supp}\left(\bar{\zeta}_{k}\right) \subseteq\left[\omega_{k-1}-\frac{\omega_{0}}{8}, \omega_{k}+\frac{\omega_{0}}{8}\right]$

- For $-n \leq k \leq-1$ :

$\operatorname{supp}\left(\zeta_{k}\right) \subseteq\left[-\omega_{k}-\frac{\omega_{0}}{8},-\omega_{k-1}+\frac{\omega_{0}}{8}\right]$

- $\sum_{j=-n}^{n} \zeta_{j} \equiv 1$ on $\left[-\omega_{+}, \omega_{+}\right]$. 
For any smooth function $\Psi: \mathcal{D} \rightarrow \mathbb{C}$ such that $\Psi(t, x)$ has compact support in $t$ for any fixed $x \in \Sigma \cap \mathcal{D}$ (having used the identification of $\mathcal{D} \backslash \mathcal{H}^{-}$with $\mathbb{R} \times(\Sigma \cap \mathcal{D})$, see Section 3.3), we will denote the Fourier transform of $\Psi$ in the $t$ coordinate by $\hat{\Psi}$, and the inverse Fourier transform in $t$ with $\check{\Psi}$.

With this notation, we define the following partition of $\psi_{t^{*}}$ (defined as (4.5)) into frequency decomposed components:

- $\psi_{\leq \omega_{+}}(t, \cdot) \doteq \int_{-\infty}^{\infty} \zeta_{\leq \omega_{+}}(\omega) \cdot e^{i \omega t} \hat{\psi}_{t^{*}}(\omega, \cdot) d \omega$

- $\psi_{\geq \omega_{+}}(t, \cdot) \doteq \int_{-\infty}^{\infty} \zeta_{\geq \omega_{+}}(\omega) \cdot e^{i \omega t} \hat{\psi}_{t^{*}}(\omega, \cdot) d \omega=\psi_{t^{*}}(t, \cdot)-\psi_{\leq \omega_{+}}(t, \cdot)$

and we decompose $\psi_{\leq \omega_{+}}$further:

- $\psi_{k}(t, \cdot) \doteq \int_{-\infty}^{\infty} \zeta_{k}(\omega) \cdot e^{i \omega t} \hat{\psi}_{\leq \omega_{+}}(\omega, \cdot) d \omega=\int_{-\infty}^{\infty} \zeta_{\leq \omega_{+}}(\omega) \cdot \zeta_{k}(\omega) \cdot e^{i \omega t} \hat{\psi}_{t^{*}}(\omega, \cdot)$ $d \omega$ for $-n \leq k \leq n$.

Note that $\psi_{\leq \omega_{+}}+\psi_{\geq \omega_{+}}=\psi_{t^{*}}$ and $\sum_{k=-n}^{n} \psi_{k}=\psi_{\leq \omega_{+}}$.

In the same way, we will decompose $F$ (defined as (4.7)) in $F_{k}, F_{\leq \omega_{+}}, F_{\geq \omega_{+}}$. Due to the linearity of the cut-off operators, we have $\square_{g} \psi_{k}=F_{k}, \square_{g} \psi_{\leq \omega_{+}}=F_{\leq \omega_{+}}$, $\square_{g} \psi_{\geq \omega_{+}}=F_{\geq \omega_{+}}$.

Note also that since $\psi_{t^{*}}$ is supported in the cylinder $\left\{r \lesssim R_{\text {sup }}+t^{*}\right\}$, the same is also true for the functions $\psi_{k}, \psi_{\leq \omega_{+}}, \psi_{\geq \omega_{+}}$.

\subsection{Bounds for the Frequency-Decomposed Components}

In this section, we will establish some useful estimates for the energy of $\psi_{k}, \psi_{\leq \omega_{+}}$, $\psi_{\geq \omega_{+}}$(as well as for the "error" terms $F_{k}, F_{\leq \omega_{+}}, F_{\geq \omega_{+}}$) in terms of the initial energy of $\psi$.

We will start by producing some basic estimates for the projection operators $\zeta_{k}, \zeta_{\leq \omega_{+}}$. Since the functions $\zeta_{\leq \omega_{+}}$and $\zeta_{k} \cdot \zeta_{\leq \omega_{+}}$are smooth with compact support, their inverse Fourier transforms $h_{\leq \omega_{+}}$and $h_{k}$ are Schwartz functions. The following lemma establishes some Schwartz bounds for $h_{\leq \omega_{+}}, h_{k}$. In view of the fact that

$$
\psi_{\leq \omega_{+}}(t, \cdot)=\int_{-\infty}^{\infty} h_{\leq \omega_{+}}(t-s) \cdot \psi_{t^{*}}(s, \cdot) d s,
$$

and for $-n \leq k \leq n$ :

$$
\psi_{k}(t, \cdot)=\int_{-\infty}^{\infty} h_{k}(t-s) \cdot \psi_{t^{*}}(s, \cdot) d s,
$$

the Schwartz bounds for $h_{\leq \omega_{+}}$and $h_{k}$ will then be used to establish useful estimates for the functions $\psi_{k}, \psi_{\leq \omega_{+}}, \psi_{\geq \omega_{+}}$.

Lemma 4.1 For $\omega_{0} \leq 1$ and $\omega_{+} \geq 1$, the convolution kernels $h_{\leq \omega_{+}}$and $h_{k}$ (for $-n \leq k \leq n$ ) satisfy:

$$
\sup _{t}\left(\omega_{+}^{-1}\left(1+\left|\omega_{+} t\right|\right)^{q}\left|h_{\leq \omega_{+}}(t)\right|\right) \leq C_{q}
$$


and

$$
\sup _{t}\left(\omega_{0}^{-1}\left(1+\left|\omega_{0} t\right|\right)^{q}\left|h_{k}(t)\right|\right) \leq C_{q}
$$

Proof From the definition $\zeta_{\leq \omega_{+}}(\omega)=\chi_{3}\left(\frac{\omega}{\omega_{+}}\right)$, we compute that $h_{\leq \omega_{+}}(t)=\omega_{+}$. $\check{\chi}_{3}\left(\omega_{+} t\right)$, where $\check{\chi}_{3}$ is a Schwartz function being the inverse Fourier transform of the compactly supported $\chi_{3}$. Hence, we can bound for each $q \in \mathbb{N}$ :

$$
\sup _{t}\left(\omega_{+}^{-1}\left(1+\left|\omega_{+} t\right|\right)^{q}\left|h_{\leq \omega_{+}}(t)\right|\right) \leq C_{q} .
$$

Similarly, due to the definition of $\zeta_{k}(4.12)$ and the bounds $\frac{1}{4} \omega_{0}<\omega_{k+1}-\omega_{k}<$ $\frac{1}{2} \omega_{0}$, we also have for each $q \in \mathbb{N}$ :

$$
\int_{-\infty}^{\infty}\left|\frac{d^{q}}{d \omega^{q}}\left\{\zeta_{k} \cdot \zeta_{\leq \omega_{+}}\right\}(\omega)\right| d \omega \leq C_{q} \cdot \sum_{i=0}^{q} \int_{-\infty}^{\infty}\left|\zeta_{k}^{(i)}(\omega)\right| \cdot\left|\zeta_{\leq \omega_{+}}^{(q-i)}(\omega)\right| d \omega \leq C_{q} \omega_{0}^{1-q}
$$

In the above, the constant $C_{q}$ does not depend on $k$, since $\sup _{\omega}\left|\zeta_{\leq \omega_{+}}^{(l)}(\omega)\right| \leq C_{l} \omega_{+}^{-l} \leq$ $C_{l}$ and $\int_{-\infty}^{\infty}\left|\zeta_{k}^{(l)}(\omega)\right| d \omega \leq C_{l} \cdot \omega_{0}^{-l+1}$.

Thus, since $h_{k}=\left(\zeta_{k} \cdot \check{\zeta}_{\leq \omega_{+}}\right)$, we can bound for each $q \in \mathbb{N},-n \leq k \leq n$ :

$$
\sup _{t}\left|\left(\omega_{0} t\right)^{q} \cdot h_{k}(t)\right| \leq C \cdot \int_{-\infty}^{\infty} \omega_{0}^{q}\left|\frac{d^{q}}{d \omega^{q}}\left\{\zeta_{k} \cdot \zeta_{\leq \omega_{+}}\right\}(\omega)\right| d \omega \leq C_{q} \omega_{0}^{1-q+q}=C_{q} \omega_{0}
$$

and hence for any $q \in \mathbb{N}$ we obtain the desired estimate:

$$
\sup _{t}\left(\omega_{0}^{-1}\left(1+\left|\omega_{0} t\right|\right)^{q}\left|h_{k}(t)\right|\right) \leq C_{q} .
$$

Let us also state a straightforward lemma that will be used frequently throughout this paper:

Lemma 4.2 For any $q \geq 0$, there exist constants $c_{q}, C_{q}$, such that for any solution $\psi$ to the wave equation $\square_{g} \psi=0$ on $\mathcal{D}$ and any $\tau \in \mathbb{R}$ we can bound

$$
c_{q} \cdot \int_{\left\{t_{-}=\tau\right\}} r^{q} \cdot J_{\mu}^{N}(\psi) n^{\mu} \leq \int_{\{t=\tau\}} r^{q} \cdot J_{\mu}^{N}(\psi) n^{\mu} \leq C_{q} \cdot \int_{\left\{t_{-}=\tau\right\}} r^{q} \cdot J_{\mu}^{N}(\psi) n^{\mu} .
$$

In paticular, for $q=0$ we have the identity

$$
\int_{\left\{t_{-}=\tau\right\}} J_{\mu}^{N}(\psi) n^{\mu}=\int_{\{t=\tau\}} J_{\mu}^{N}(\psi) n^{\mu} .
$$

Proof As we remarked earlier, $\left\{t_{-}=\tau\right\} \cap\left\{r \leq R_{1}\right\} \equiv\{t=\tau\} \cap\left\{r \leq R_{1}\right\}$, and $N \equiv T$ for $r \geq R_{1}$. Hence, since $T$ is Killing and $\psi$ solves the equation $\square_{g} \psi=0$, the current $J_{\mu}^{N}(\psi)=T_{\mu \nu}(\psi) N^{\nu}$ is divergence free for $r \geq R_{1}$. Integrating, therefore, 
$\nabla^{\mu} J_{\mu}^{N}(\psi)=0$ in the domain bounded by $\left\{t_{-}=\tau\right\}$ and $\{t=\tau\}$ which lies entirely in the region $\left\{r \geq R_{1}\right\}$ ), identity (4.21) follows immediately.

In order to show (4.20), we define for $l \in \mathbb{Z}$ the intervals $I_{l}=\left[2^{l}, 2^{l+1}\right] \subseteq \mathbb{R}_{+}$, for $l \geq 1, I_{0}=[0,2]$ for $l=0$, and $I_{l}=\varnothing$ for $l<0$. We then compute that

$$
\int_{t=\tau} r^{q} \cdot J_{\mu}^{N}(\psi) n^{\mu} \sim_{q} \sum_{l=0}^{\infty} 2^{q \cdot l} \cdot \int_{\{t=\tau\} \cap\left\{r \in I_{l}\right\}} J_{\mu}^{N}(\psi) n^{\mu},
$$

and similarly

$$
\int_{t_{-}=\tau} r^{q} \cdot J_{\mu}^{N}(\psi) n^{\mu} \sim_{q} \sum_{l=0}^{\infty} 2^{q \cdot l} \cdot \int_{\left\{t_{-}=\tau\right\} \cap\left\{r \in I_{l}\right\}} J_{\mu}^{N}(\psi) n^{\mu} .
$$

Since $t_{-}=t+\frac{1}{2} \chi_{1}(r)\left(r-R_{1}\right)$, we deduce that there exists an integer $Z>0$ such that for every $\tau \in \mathbb{R}$ and every $l \in \mathbb{N}$ :

$$
J^{+}\left(\left\{t_{-}=\tau\right\} \cap\left\{r \in I_{l}\right\}\right) \cap\{t=\tau\} \subseteq\{t=\tau\} \cap\left\{r \in \cup_{i=l-Z}^{l+Z} I_{i}\right\}
$$

and

$$
J^{-}\left(\{t=\tau\} \cap\left\{r \in I_{l}\right\}\right) \cap\left\{t_{-}=\tau\right\} \subseteq\left\{t_{-}=\tau\right\} \cap\left\{r \in \cup_{i=l-Z}^{l+Z} I_{i}\right\} .
$$

Since $\left\{t_{-}=\tau\right\} \equiv\{t=\tau\}$ for $r \leq R_{1}$ and $T \equiv N$ for $r \geq R_{1}$, the inclusions (4.24) and (4.25) imply, after integrating the identity $\nabla^{\mu} J_{\mu}^{T}=0$ on $J^{-}(\{t=\tau\} \cap\{r \in$ $\left.\left.I_{l}\right\}\right) \cap J^{+}\left(\left\{t_{-}=\tau\right\}\right)$, that for any $l$ :

$$
\int_{\{t=\tau\} \cap\left\{r \in I_{l}\right\}} J_{\mu}^{N}(\psi) n^{\mu} \leq \int_{\left\{t_{-}=\tau\right\} \cap\left\{r \in \cup_{i=l-Z}^{l+Z} I_{i}\right\}} J_{\mu}^{N}(\psi) n^{\mu}
$$

and similarly, after an integration on $J^{+}\left(\left\{t_{-}=\tau\right\} \cap\left\{r \in I_{l}\right\}\right) \cap J^{-}(\{t=\tau\})$ :

$$
\int_{\left\{t_{-}=\tau\right\} \cap\left\{r \in I_{l}\right\}} J_{\mu}^{N}(\psi) n^{\mu} \leq \int_{\{t=\tau\} \cap\left\{r \in \cup_{i=l-Z}^{l+Z} I_{i}\right\}} J_{\mu}^{N}(\psi) n^{\mu} .
$$

Thus, in view of (4.22), (4.23), (4.26) and (4.27) we can bound:

$$
\begin{aligned}
\int_{t=\tau} r^{q} \cdot J_{\mu}^{N}(\psi) n^{\mu} & \leq C_{q} \cdot \sum_{l=0}^{\infty} 2^{q l} \cdot \int_{\{t=\tau\} \cap\left\{r \in I_{l}\right\}} J_{\mu}^{N}(\psi) n^{\mu} \\
& \leq C_{q} \cdot \sum_{l=0}^{\infty} 2^{q l} \cdot \int_{\left\{t_{-}=\tau\right\} \cap\left\{r \in \cup_{i=l-Z}^{l+Z} I_{i}\right\}} J_{\mu}^{N}(\psi) n^{\mu} \\
& \leq 2 Z C_{q} \cdot \sum_{l=0}^{\infty} 2^{q(l+Z+1)} \cdot \int_{\left\{t_{-}=\tau\right\} \cap\left\{r \in I_{l}\right\}} J_{\mu}^{N}(\psi) n^{\mu}
\end{aligned}
$$




$$
\begin{aligned}
& =C_{q} \cdot 2 Z \cdot 2^{q(Z+1)} \sum_{l=0}^{\infty} 2^{q l} \cdot \int_{\left\{t_{-}=\tau\right\} \cap\left\{r \in I_{l}\right\}} J_{\mu}^{N}(\psi) n^{\mu} \\
& \leq C_{q} \cdot \int_{t_{-}=\tau} r^{q} \cdot J_{\mu}^{N}(\psi) n^{\mu}
\end{aligned}
$$

and similarly

$$
\begin{aligned}
\int_{t_{-}=\tau} r^{q} \cdot J_{\mu}^{N}(\psi) n^{\mu} & \leq C_{q} \cdot \sum_{l=0}^{\infty} 2^{q l} \cdot \int_{\left\{t_{-}=\tau\right\} \cap\left\{r \in I_{l}\right\}} J_{\mu}^{N}(\psi) n^{\mu} \\
& \leq C_{q} \cdot \sum_{l=0}^{\infty} 2^{q l} \cdot \int_{\{t=\tau\} \cap\left\{r \in \cup_{i=l-Z}^{l+Z} I_{i}\right\}} J_{\mu}^{N}(\psi) n^{\mu} \\
& \leq 2 Z C_{q} \cdot \sum_{l=0}^{\infty} 2^{q(l+Z+1)} \cdot \int_{\{t=\tau\} \cap\left\{r \in I_{l}\right\}} J_{\mu}^{N}(\psi) n^{\mu} \\
& \leq C_{q} \cdot \int_{t=\tau} r^{q} \cdot J_{\mu}^{N}(\psi) n^{\mu}
\end{aligned}
$$

thus reaching the desired inequality

$$
c_{q} \cdot \int_{t_{-}=\tau} r^{q} \cdot J_{\mu}^{N}(\psi) n^{\mu} \leq \int_{t=\tau} r^{q} \cdot J_{\mu}^{N}(\psi) n^{\mu} \leq C_{q} \cdot \int_{t_{-}=\tau} r^{q} \cdot J_{\mu}^{N}(\psi) n^{\mu} .
$$

We will now establish some estimates for the error terms $F_{k}, F_{\leq \omega_{+}}, F_{\geq \omega_{+}}$. Note that from now on we will always assume without loss of generality that $\omega_{0} \leq 1$ and $\omega_{+} \geq 1$.

Lemma 4.3 We can bound for any $q, q^{\prime} \in \mathbb{N}$ and any $0 \leq t_{1} \leq t_{2} \leq t^{*}$ :

$$
\int_{\mathcal{R}\left(t_{1}, t_{2}\right)} r^{q}\left|F_{k}\right|^{2} \leq C_{q, q^{\prime}}\left(\omega_{0}\right) \cdot\left(\left(1+t_{1}\right)^{-q^{\prime}}+\left(1+t^{*}-t_{2}\right)^{-q^{\prime}}\right) \int_{t=0} J_{\mu}^{N}(\psi) n^{\mu}
$$

for $-n \leq k \leq n$. The same inequality also holds for $F_{\leq \omega_{+}}, F_{\geq \omega_{+}}$in place of $F_{k}$.

Proof Since $F_{k}(t, \cdot)=\int_{-\infty}^{\infty} h_{k}(t-s) \cdot F(s, \cdot) d s$, we can bound $(d x$ denoting in the next lines the $d g_{\Sigma}$ integration measure):

$$
\begin{aligned}
\int_{\mathcal{R}\left(t_{1}, t_{2}\right)} r^{q}\left|F_{k}\right|^{2} \leq & C \cdot \int_{\Sigma_{0}} \int_{t_{1}}^{t_{2}} r^{q}\left|\int_{-\infty}^{\infty} h_{k}(t-s) \cdot F(s, x) d s\right|^{2} d t d x \\
\leq & C \cdot R_{1}^{q} \cdot\left(\int_{\Sigma_{0} \cap\left\{r \leq R_{1}\right\}} \int_{t_{1}}^{t_{2}}\left|\int_{-\infty}^{\infty} h_{k}(t-s) \cdot F(s, x) d s\right|^{2} d t d x\right) \\
& +\int_{\Sigma_{0} \cap\left\{r \geq R_{1}\right\}} r^{q} \int_{t_{1}}^{t_{2}}\left|\int_{-\infty}^{\infty} h_{k}(t-s) \cdot F(s, x) d s\right|^{2} d t d x .
\end{aligned}
$$


Because in the region $\left\{r \leq R_{1}\right\}, F=\partial^{\mu} h_{t^{*}} \cdot \partial_{\mu} \psi+\left(\square_{g} h_{t^{*}}\right) \cdot \psi$ is supported in $\{0 \leq t \leq 1\} \cup\left\{t^{*}-1 \leq t \leq t^{*}\right\}$ and $\sup _{t}\left|\omega_{0}^{-1}\left(1+\left|\omega_{0} t\right|\right)^{q^{\prime}+1} h_{k}(t)\right| \leq C_{q^{\prime}+1}$ due to (4.16), the first term of the right hand side of (4.32) can be bounded by

$$
\begin{aligned}
& \int_{\Sigma_{0} \cap\left\{r \leq R_{1}\right\}} \int_{t_{1}}^{t_{2}}\left|\int_{-\infty}^{\infty} h_{k}(t-s) \cdot F(s, x) d s\right|^{2} d t d x \\
& \leq \int_{\Sigma_{0} \cap\left\{r \leq R_{1}\right\}} \int_{t_{1}}^{t_{2}}\left|\int_{[0,1] \cup\left[t^{*}-1, t^{*}\right]} \frac{C_{q^{\prime}} \omega_{0}}{\left(1+\omega_{0}|t-s|\right)^{q^{\prime}+1}} \cdot F(s, x) d s\right|^{2} d t d x \\
& \leq C_{q^{\prime}}\left(\omega_{0}\right) \int_{\Sigma_{0} \cap\left\{r \leq R_{1}\right\}} \int_{t_{1}}^{t_{2}}\left\{(1+|t|)^{-q^{\prime}-1} \int_{[0,1]}|F(s, x)|^{2} d s\right. \\
& \left.\quad+\left(1+\left|t^{*}-t\right|\right)^{-q^{\prime}-1} \int_{\left[t^{*}-1, t^{*}\right]}|F(s, x)|^{2} d s\right\} d t d x \\
& \leq C_{q^{\prime}}\left(\omega_{0}\right) \cdot\left(\left(1+t_{1}\right)^{-q^{\prime}}+\left(1+t^{*}-t_{2}\right)^{-q^{\prime}}\right) \int_{t=0} J_{\mu}^{N}(\Psi) n^{\mu}
\end{aligned}
$$

the last inequality being a consequence of the boundedness assumption 4 , the fact that $F=\partial^{\mu} h_{t^{*}} \cdot \partial_{\mu} \psi+\left(\square_{g} h_{t^{*}}\right) \cdot \psi$, as well as the Hardy inequality

$$
\int_{\{t=\tau\} \cap\left\{r \leq R_{1}\right\}}|\psi|^{2} \leq C R_{1}^{2} \cdot \int_{t=\tau} J_{\mu}^{N}(\psi) n^{\mu}
$$

following from (3.16).

For the second term of (4.32), we will use the definition of $t_{+}, t_{-}$and the support of $F$ to conclude that for $r \geq R_{1}, l \in \mathbb{N}$ and $t \in\left(t_{1}, t_{2}\right)$ :

$$
\begin{aligned}
& \int_{-\infty}^{\infty}\left|h_{k}(t-s)\right| \cdot|F(s, r, \sigma)| d s=\int_{-\frac{1}{2} \chi_{1} \cdot\left(r-R_{1}\right)}^{1-\frac{1}{2} \chi_{1} \cdot\left(r-R_{1}\right)}\left|h_{k}(t-s)\right| \cdot|F(s, r, \sigma)| d s+ \\
& \quad+\int_{t^{*}-1+\frac{1}{2} \chi_{1} \cdot\left(r-R_{1}\right)}^{t^{*}+\frac{1}{2} \chi_{1} \cdot\left(r-R_{1}\right)}\left|h_{k}(t-s)\right| \cdot|F(s, r, \sigma)| d s \\
& \leq C_{l}\left(\omega_{0}\right)\left(\frac{1}{\left(1+\left|t+\frac{1}{2}\left(r-R_{1}\right)\right|\right)^{l}} \int_{-\frac{1}{2} \chi_{1} \cdot\left(r-R_{1}\right)}^{1-\frac{1}{2} \chi_{1} \cdot\left(r-R_{1}\right)}|F(s, r, \sigma)| d s\right. \\
& \left.\quad+\frac{1}{\left(1+\left|t-t^{*}-\frac{1}{2}\left(r-R_{1}\right)\right|\right)^{l}} \int_{\left.t^{*}-1+\frac{1}{2} \chi_{1} \cdot\left(r-R_{1}\right)\right)^{1 / 2}}^{t^{*}+\frac{1}{2} \chi_{1} \cdot\left(r-R_{1}\right)}|F(s, r, \sigma)| d s\right) .
\end{aligned}
$$

Therefore, by choosing $l$ large enough (with respect to $q, q^{\prime}$ ), we conclude

$$
\begin{aligned}
& \int_{\Sigma_{0} \cap\left\{r \geq R_{1}\right\}} \int_{t_{1}}^{t_{2}} r^{q}\left|\int_{-\infty}^{\infty} h_{k}(t-s) \cdot F(s, x) d s\right|^{2} d t d x \\
& \leq C_{l}\left(\omega_{0}\right) \cdot \int_{\Sigma_{0} \cap\left\{r \geq R_{1}\right\}} \int_{t_{1}}^{t_{2}} \frac{r^{q}}{\left(1+\left|t+\frac{1}{2}\left(r-R_{1}\right)\right|\right)^{2 l}}
\end{aligned}
$$




$$
\begin{aligned}
& \times\left(\int_{-\frac{1}{2} \chi_{1} \cdot\left(r-R_{1}\right)}^{1-\frac{1}{2} \chi_{1} \cdot\left(r-R_{1}\right)}|F(s, x)|^{2} d s\right) d t d x \\
& +C_{l}\left(\omega_{0}\right) \cdot \int_{\Sigma_{0} \cap\left\{r \geq R_{1}\right\}} \int_{t_{1}}^{t_{2}} \frac{r^{q}}{\left(1+\left|t-t^{*}-\frac{1}{2}\left(r-R_{1}\right)\right|\right)^{2 l}} \\
& \times\left(\int_{t^{*}-1+\frac{1}{2} \chi_{1} \cdot\left(r-R_{1}\right)}^{t^{*}+\frac{1}{2} \chi_{1} \cdot\left(r-R_{1}\right)}|F(s, x)|^{2} d s\right) d t d x \\
& \leq C_{l}\left(\omega_{0}\right) \cdot \int_{\Sigma_{0} \cap\left\{r \geq R_{1}\right\}}\left(\int_{t_{1}}^{t_{2}} \frac{r^{q}}{\left(1+\left|t+\frac{1}{2}\left(r-R_{1}\right)\right|\right)^{2 l}} d t\right) . \\
& \times\left(\int_{\frac{1}{2} \chi_{1} \cdot\left(r-R_{1}\right)}^{1-\frac{1}{2} \chi_{1} \cdot\left(r-R_{1}\right)}\left(J_{\mu}^{N}(\psi) n^{\mu}+|\psi|^{2}\right)(s, x) d s\right) d x \\
& +C_{l}\left(\omega_{0}\right) \cdot \int_{\Sigma_{0} \cap\left\{r \geq R_{1}\right\}}\left(\int_{t_{1}}^{t_{2}} \frac{r^{q}}{\left(1+\left|t-t^{*}-\frac{1}{2}\left(r-R_{1}\right)\right|\right)^{2 l}} d t\right) . \\
& \times\left(\int_{t^{*}-1+\frac{1}{2} \chi_{1} \cdot\left(r-R_{1}\right)}^{t^{*}+\frac{1}{2} \chi_{1} \cdot\left(r-R_{1}\right)}\left(J_{\mu}^{N}(\psi) n^{\mu}+|\psi|^{2}\right)(s, x) d s\right) d x \\
& \leq C_{q, q^{\prime}}\left(\omega_{0}\right) \cdot\left(1+t_{1}\right)^{-q^{\prime}} \int_{\Sigma_{0} \cap\left\{r \geq R_{1}\right\}} \frac{1}{1+r^{4}} \\
& \times\left(\int_{-\frac{1}{2} \chi_{1} \cdot\left(r-R_{1}\right)}^{1-\frac{1}{2} \mathrm{\chi}_{1} \cdot\left(r-R_{1}\right)}\left(J_{\mu}^{N}(\psi) n^{\mu}+|\psi|^{2}\right)(s, x) d s\right) d x \\
& +C_{q, q^{\prime}}\left(\omega_{0}\right) \cdot\left(1+t^{*}-t_{2}\right)^{-q^{\prime}} \int_{\Sigma_{0} \cap\left\{r \geq R_{1}\right\}} \frac{1}{1+r^{4}} \\
& \times\left(\int_{t^{*}-1+\frac{1}{2} \chi_{1} \cdot\left(r-R_{1}\right)}^{t^{*}+\frac{1}{2} \chi_{1} \cdot\left(r-R_{1}\right)}\left(J_{\mu}^{N}(\psi) n^{\mu}+|\psi|^{2}\right)(s, x) d s\right) d x \\
& \leq C_{q, q^{\prime}}\left(\omega_{0}\right)\left(1+t_{1}\right)^{-q^{\prime}} \cdot \int_{0}^{\infty} \frac{1}{1+\tau^{2}} \\
& \times \int_{\left\{t^{-}=\tau\right\} \cap\left\{R_{1} \lesssim r \lesssim R_{1}+\tau\right\}}\left(J_{\mu}^{N}(\psi) n^{\mu}+\frac{1}{r^{2}}|\psi|^{2}\right) d x d \tau \\
& +C_{q, q^{\prime}}\left(\omega_{0}\right)\left(1+t^{*}-t_{2}\right)^{-q^{\prime}} \int_{0}^{1} \int_{\left\{t^{-}=\tau\right\} \cap\left\{r \geq R_{1}\right\}} \frac{1}{r^{2}}\left(J_{\mu}^{N}(\psi) n^{\mu}+\frac{1}{r^{2}}|\psi|^{2}\right) d x d \tau \\
& \leq C_{q, q^{\prime}}\left(\omega_{0}\right)\left(\left(1+t_{1}\right)^{-q^{\prime}}+\left(1+t^{*}-t_{2}\right)^{-q^{\prime}}\right) \int_{t=0} J_{\mu}^{N}(\psi) n^{\mu},
\end{aligned}
$$

the last inequality being a consequence of the boundedness assumption 4 and Lemma 4.2 (and a Hardy inequality for the $\frac{1}{r^{2}}|\psi|^{2}$ term). Thus, from (4.32), (4.33) and (4.36) we obtain the desired bound:

$$
\int_{\mathcal{R}\left(t_{1}, t_{2}\right)} r^{q}\left|F_{k}\right|^{2} \leq C_{q, q^{\prime}}\left(\omega_{0}\right)\left(\left(1+t_{1}\right)^{-q^{\prime}}+\left(1+t^{*}-t_{2}\right)^{-q^{\prime}}\right) \int_{t=0} J_{\mu}^{N}(\psi) n^{\mu} .
$$


In the same way, one can show the same inequality for $F_{\leq \omega_{+}}, F_{\geq \omega_{+}}=F-F_{\leq \omega_{+}}$.

We will also need to bound the energy of the frequency-decomposed components of $\psi$ in terms of the energy of $\psi$ itself:

Lemma 4.4 There exists a positive constant $C\left(\omega_{0}\right)$ such that for any $-n \leq k \leq n$, and any $\tau \in\left[0, t^{*}\right]$ :

$$
\int_{t=\tau} J_{\mu}^{N}\left(\psi_{k}\right) n^{\mu} \leq C\left(\omega_{0}\right) \cdot \int_{t=0} J_{\mu}^{N}(\psi) n^{\mu} .
$$

The same estimate holds for $\psi_{\leq \omega_{+}}, \psi_{\geq \omega_{+}}$in place of $\psi_{k}$ (and in that case the constant in (4.40) does not depend on $\left.\omega_{0}\right)$.

Proof The proof will be similar to the proof of the previous lemma. Since $\nabla_{\mu} \psi_{k}(t, \cdot)=$ $\int_{-\infty}^{\infty} h_{k}(t-s) \cdot \nabla_{\mu} \psi_{t^{*}}(s, \cdot) d s$, we can bound:

$$
\int_{t=\tau} J_{\mu}^{N}\left(\psi_{k}\right) n^{\mu} \leq C \cdot \int_{t=\tau}\left(\left|T \psi_{k}\right|^{2}+\left|\nabla_{\Sigma_{\tau}} \psi_{k}\right|_{g_{t}}^{2}\right),
$$

where $g_{t}$ is the induced Riemannian metric on $\Sigma_{\tau}$. We can also estimate for $q$ large enough

$$
\begin{aligned}
\int_{t=\tau}\left|T \psi_{k}\right|^{2} & =\int_{t=\tau}\left|\int_{-\infty}^{\infty} h_{k}(t-s) \cdot T \psi_{t^{*}}(s, \cdot) d s\right|^{2} \\
& \leq C_{q} \cdot \omega_{0}^{2} \int_{t=\tau}\left(\int_{-\infty}^{\infty} \frac{1}{1+\left(\omega_{0}|t-s|\right)^{q}}\left|T \psi_{t^{*}}(s, \cdot)\right| d s\right)^{2} \\
& \leq C_{q} \cdot \omega_{0} \int_{t=\tau}\left(\int_{-\infty}^{\infty} \frac{1}{1+\left(\omega_{0}|t-s|\right)^{q}}\left|T \psi_{t^{*}}(s, \cdot)\right|^{2} d s\right) \\
& \leq C_{q} \cdot \omega_{0} \int_{-\infty}^{\infty} \frac{1}{1+\left(\omega_{0}|\tau-s|\right)^{q}}\left(\int_{t=s}\left|T \psi_{t^{*}}\right|^{2}\right) d s .
\end{aligned}
$$

We now recall that $T \psi_{t^{*}}=h_{t^{*}} \cdot T \psi+T h_{t^{*}} \cdot \psi$. We note that $h_{t^{*}}$ is supported only in $\left\{t_{-} \geq 0\right\}$, and hence

$$
\int_{\{t=s\}}\left|h_{t^{*}} \cdot T \psi\right|^{2}=\int_{\{t=s\} \cap\left\{t_{-} \geq 0\right\}}\left|h_{t^{*}} \cdot T \psi\right|^{2} .
$$

Therefore, for $s \geq 0$, the boundedness assumption 4 implies that

$$
\int_{\{t=s\}}\left|h_{t^{*}} \cdot T \psi\right|^{2} \leq C \cdot \int_{\{t=0\}} J_{\mu}^{N}(\psi) n^{\mu} .
$$

For $s<0$, integrating $\nabla^{\mu} J_{\mu}^{T}$ in the domain bounded by $\{t=s\} \cap\left\{t_{-} \geq 0\right\}$ and $\left\{t_{-}=0\right\}$ we obtain 


$$
\int_{\{t=s\} \cap\left\{t_{-} \geq 0\right\}}\left|h_{t^{*}} \cdot T \psi\right|^{2} \leq C \cdot \int_{t_{-}=0} J_{\mu}^{N}(\psi) n^{\mu} .
$$

Thus, applying in this case Lemma 4.2 , we deduce that for any $s \in \mathbb{R}$ we can bound

$$
\int_{t=s}\left|h_{t^{*}} \cdot T \psi\right|^{2} \leq C \cdot \int_{t=0} J_{\mu}^{N}(\psi) n^{\mu}
$$

Moreover, $\operatorname{grad}\left(h_{t^{*}}\right)$ is only supported in $\left\{0 \leq t_{-} \leq 1\right\} \cup\left\{t^{*}-1 \leq t_{+} \leq t^{*}\right\}$, and thus we can bound through a Hardy inequality (in view of the definition (4.1) and (4.2) of $t_{+}, t_{-}$) for any $s \in \mathbb{R}$

$$
\int_{t=s}\left|T h_{t^{*}} \cdot \psi\right|^{2} \leq C \cdot\left(1+\operatorname{dist}\left\{s,\left[0, t^{*}\right]\right\}\right)^{2} \cdot \int_{\{t=s\} \cap\left\{t_{-} \geq 0\right\}} J_{\mu}^{N}(\psi) n^{\mu} .
$$

For $s \geq 0$, Assumption 4 states that $\int_{t=s} J_{\mu}^{N}(\psi) n^{\mu} \leq C \cdot \int_{t=0} J_{\mu}^{N}(\psi) n^{\mu}$. For $s<0$, the conservation of the $J^{T}$ current in the domain bounded by $\{t=s\} \cap\left\{t_{-} \geq 0\right\}$ and $\left\{t_{-}=\right.$ $0\}$ together with Lemma 4.2 imply that $\int_{\{t=s\} \cap\left\{t_{-} \geq 0\right\}} J_{\mu}^{N}(\psi) n^{\mu} \leq C \cdot \int_{t=0} J_{\mu}^{N}(\psi) n^{\mu}$ in this case as well. Thus, (4.45) yields

$$
\int_{t=s}\left|T h_{t^{*}} \cdot \psi\right|^{2} \leq C \cdot\left(1+\operatorname{dist}\left\{s,\left[0, t^{*}\right]\right\}\right)^{2} \cdot \int_{t=0} J_{\mu}^{N}(\psi) n^{\mu}
$$

Substituting (4.44) and (4.46) in 4.40, we obtain

$$
\int_{t=\tau}\left|T \psi_{k}\right|^{2} \leq C_{q} \cdot \omega_{0}\left\{\int_{-\infty}^{\infty} \frac{\left(1+\operatorname{dist}\left\{s,\left[0, t^{*}\right]\right\}\right)^{2}}{1+\left(\omega_{0}|\tau-s|\right)^{q}} d s\right\} \int_{t=0} J_{\mu}^{N}(\psi) n^{\mu}
$$

and hence, since $\tau \in\left[0, t^{*}\right]$, if we fix $q=4$ we conclude

$$
\int_{t=\tau}\left|T \psi_{k}\right|^{2} \leq C\left(\omega_{0}\right) \int_{t=0} J_{\mu}^{N}(\psi) n^{\mu}
$$

In the same way, we can estimate

$$
\int_{t=\tau}\left|\nabla_{\Sigma_{\tau}} \psi_{k}\right|^{2} \leq C\left(\omega_{0}\right) \int_{t=0} J_{\mu}^{N}(\psi) n^{\mu}
$$

and thus attain (4.38) regarding $\psi_{k}$.

The statement about $\psi_{\leq \omega_{+}}$follows in exactly the same way by considering $h_{\leq \omega_{+}}$ instead of $h_{k}$, and using (4.15) instead of (4.16). In this case, in view of the fact that $\omega_{+} \geq 1$, we can also make the constant appearing in (4.58) (which in the case of $\psi_{k}$ is $\sim \omega_{0}^{-2}$ ) not to depend on $\omega_{0}$, but we have chosen to neglect this fact.

Finally, the statement for $\psi_{\geq \omega_{+}}=\psi_{t^{*}}-\psi_{\leq \omega_{+}}$follows immediately, in view also of the boundedness assumption 4 and the support of the cut-off function $h_{t^{*}}$, which allow us to handle the $\psi_{t^{*}}$ term in exactly the same way as we did for $\psi_{k}$. 
It would seem useful to estimate the energy of $\psi_{k}$ (and $\psi_{\leq \omega_{+}}, \psi_{\geq \omega_{+}}$as well) only in terms of its own initial data, and not the initial data of $\bar{\psi}$. Unfortunately, such a bound is not obtainable. We can instead establish the following estimate:

Lemma 4.5 For any $q \in \mathbb{N}$, there exist suitable constants $C, C_{q}\left(\omega_{0}\right)$ such that for any $-n \leq k \leq n$, any $0 \leq t_{s t} \leq \frac{1}{2} t^{*}$ and any $t_{s t} \leq t_{1} \leq t_{2} \leq t^{*}-t_{s t}$ satisfying $t_{2}-t_{1} \leq t_{s t}$, the following inequality holds for any $\tau \in\left[t_{1}, t_{2}\right]$ :

$$
\int_{t=\tau} J_{\mu}^{N}\left(\psi_{k}\right) n^{\mu} \leq C \cdot \int_{t=t_{1}} J_{\mu}^{N}\left(\psi_{k}\right) n^{\mu}+C_{q}\left(\omega_{0}\right)\left(1+t_{s t}\right)^{-q} \int_{t=0} J_{\mu}^{N}(\psi) n^{\mu} .
$$

The same also holds for $\psi_{\leq \omega_{+}}, \psi_{\geq \omega_{+}}$in place of $\psi_{k}$.

Proof Recall that $\psi_{k}$ satisfies $\square_{g} \psi_{k}=F_{k}$. Therefore, on $J^{+}\left(\left\{t=t_{1}\right\}\right) \cap \mathcal{D}$ we can uniquely decompose $\psi_{k}=\psi_{k, \text { hom }}+\psi_{k, \text { inhom }}$, where

$$
\left\{\begin{array}{l}
\square_{g} \psi_{k, h o m}=0 \\
\left.\psi_{k, h o m}\right|_{t=t_{1}}=\left.\psi_{k}\right|_{t=t_{1}} \\
\left.\partial_{t} \psi_{k, h o m}\right|_{t=t_{1}}=\left.\partial_{t} \psi_{k}\right|_{t=t_{1}}
\end{array}\right.
$$

and

$$
\left\{\begin{array}{l}
\square_{g} \psi_{k, \text { inhom }}=F_{k} \\
\left.\psi_{k, \text { inhom }}\right|_{t=t_{1}}=0 \\
\left.\partial_{t} \psi_{k, \text { inhom }}\right|_{t=t_{1}}=0 .
\end{array}\right.
$$

The boundedness assumtion 4 applies to $\psi_{k, h o m}$ and we obtain (since the initial data on $t=t_{1}$ that $\psi_{k, h o m}$ satisfies are the ones induced by $\psi_{k}$ )

$$
\int_{t=\tau} J_{\mu}^{N}\left(\psi_{k, h o m}\right) n^{\mu} \leq C \cdot \int_{t=t_{1}} J_{\mu}^{N}\left(\psi_{k}\right) n^{\mu}
$$

For $\psi_{k, \text { inhom }}$, we will use Duhamel's principle as follows: For $s \geq t_{1}$, let $u_{s}$ : $J^{+}(\{t=s\}) \cap \mathcal{D} \rightarrow \mathbb{C}$ be the unique solution to the initial value problem $\square_{g} u_{s}=0$, $\left.u_{s}\right|_{t=s}=0,\left.\partial_{t} u_{s}\right|_{t=s}=\left.F_{k}\right|_{t=s}$. Then the following relation holds

$$
\psi_{k, \text { inhom }}(t, \cdot)=\int_{t_{1}}^{t} u_{s}(t, \cdot) d s
$$

This can be deduced by just noting that (in view of the fact that the vector field $T$ is Killing), the function $\Psi(t, x)=\psi_{k, \text { inhom }}(t, x)-\int_{t_{1}}^{t} u_{s}(t, x) d s$ (for any $t \in \mathbb{R}$ and $x \in \Sigma \cap \mathcal{D})$ satisfies on $J^{+}\left(\left\{t=t_{1}\right\}\right) \cap \mathcal{D}$ :

$$
\square_{g} \Psi=F_{k}(t, x)-F_{k}(t, x)=0,
$$

with $\left.\Psi\right|_{t=t_{1}}=0,\left.\partial_{t} \Psi\right|_{t=t_{1}}=0$. Thus, $\Psi \equiv 0$ on $J^{+}\left(\left\{t=t_{1}\right\}\right) \cap \mathcal{D}$. 
Therefore, since the boundedness assumption 4 applies to $u_{s}$, yielding for $\tau \geq s$

$$
\int_{t=\tau} J_{\mu}^{N}\left(u_{s}\right) n^{\mu} \leq C \cdot \int_{t=s}\left|F_{k}\right|^{2}
$$

we infer that:

$$
\int_{t=\tau} J_{\mu}^{N}\left(\Psi_{k, \text { inhom }}\right) n^{\mu} \leq C \cdot \int_{t=0}\left(\int_{t_{1}}^{\tau}\left|\partial u_{s}(\tau, \cdot)\right| d s\right)^{2} d x \leq C \cdot\left(\tau-t_{1}\right) \cdot \int_{R\left(t_{1}, \tau\right)}\left|F_{k}\right|^{2} .
$$

In view of Lemma 4.3, and the fact that $t_{s t} \leq t_{1} \leq \tau \leq t_{2} \leq t^{*}-t_{s t}$ and $\tau-t_{1} \leq$ $t_{2}-t_{1} \leq t_{s t}$, from (4.56) we conclude that

$$
\int_{t=\tau} J_{\mu}^{N}\left(\psi_{k, \text { inhom }}\right) n^{\mu} \leq C_{q}\left(\omega_{0}\right) \cdot\left(1+t_{s t}\right)^{-q} \int_{t=0} J_{\mu}^{N}(\psi) n^{\mu} .
$$

Since $\psi_{k}=\psi_{k, \text { hom }}+\psi_{k, \text { inhom }}$, adding 4.53 and 4.57 yields 4.50 . The statement about $\psi_{\leq \omega_{+}}, \psi_{\geq \omega_{+}}$follows in exactly the same way.

We will also need to use a boundedness statement for the energy of $\psi_{k}, \psi_{\leq \omega_{+}}$and $\psi_{\geq \omega_{+}}$on spacelike hypersurfaces more general than $\{t=$ const $\}$. The following lemma, is a straightforward generalisation of Lemma 4.5, and its proof is identical (and will be omitted).

Lemma 4.6 Let $\vartheta: \Sigma_{0} \rightarrow \mathbb{R}$ be a non negative function such that $t_{\vartheta}=t-\vartheta$ has spacelike level sets. Then for any $q \in \mathbb{N}$, there exist suitable constants $C, C_{q}\left(\omega_{0}\right)$ (depending also on the precise choice of $\vartheta$ ) such that for any $-n \leq k \leq n$, any $0 \leq t_{s t} \leq \frac{1}{2} t^{*}$ and any $t_{s t} \leq t_{1} \leq t_{2} \leq t^{*}-t_{\text {st }}$ satisfying $t_{2}-t_{1} \leq t_{s t}$, the following inequality holds for any $\tau \in\left[t_{1}, t_{2}\right]$ :

$$
\begin{aligned}
& \int_{\left\{t_{\vartheta}=\tau\right\} \cap \mathcal{R}\left(0, t^{*}\right)} J_{\mu}^{N}\left(\psi_{k}\right) n^{\mu} \leq C \cdot \int_{\left\{t_{\vartheta}=t_{1}\right\} \cap \mathcal{R}\left(0, t^{*}\right)} J_{\mu}^{N}\left(\psi_{k}\right) n^{\mu} \\
& +C_{q}\left(\omega_{0}\right)\left(1+t_{s t}\right)^{-q} \int_{t=0} J_{\mu}^{N}(\psi) n^{\mu} .
\end{aligned}
$$

The same estimate also holds for $\psi_{\leq \omega_{+}}, \psi_{\geq \omega_{+}}$in place of $\psi_{k}$.

We will also need to localise in time estimates of the form $\int_{-\infty}^{\infty}\left|\partial_{t} \psi_{k}\right|^{2} d t \sim$ $\omega_{k}^{2} \int_{-\infty}^{\infty}\left|\psi_{k}\right|^{2} d t$. To this end, we have to introduce a few more cut off functions.

Let $\bar{\chi}_{3}: \mathbb{R} \rightarrow[0,1]$ be a smooth function that is identically 1 on $\left[-\frac{9}{8}, \frac{9}{8}\right]$ and identically 0 outside $\left(-\frac{5}{4}, \frac{5}{4}\right)$. Then for $1 \leq k \leq n$ we will define the functions

$$
\bar{\zeta}_{k}=\bar{\chi}_{3}\left(\frac{\omega-\frac{\omega_{k-1}+\omega_{k}}{2}}{\frac{1}{2}\left(\omega_{k}-\omega_{k-1}\right)}\right) .
$$

We will extend this definition for $-n \leq k \leq-1$ by setting $\bar{\zeta}_{k}(\omega)=\bar{\zeta}_{-k}(-\omega)$. 
Note that the $\bar{\zeta}_{k}$ 's are smooth functions with compact support, and hence their inverse Fourier transforms $\bar{h}_{k}$ are Schwartz functions. For $1 \leq k \leq n$, the $\bar{h}_{k}$ 's are of the form

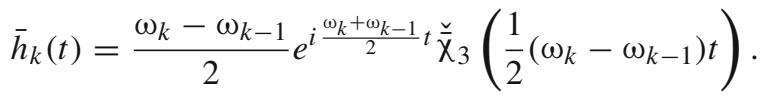

Hence, due to the bound $\frac{1}{4} \omega_{0}<\omega_{k}-\omega_{k-1}<\frac{1}{2} \omega_{0}$, the fact that $\check{\bar{\chi}}_{3}$ is a Schwartz function implies that for any $q \in \mathbb{N}$

$$
\begin{gathered}
\sup _{t}\left(\omega_{0}^{-1}\left\{1+\left|\omega_{0} t\right|^{q}\right\}\left|\bar{h}_{k}(t)\right|\right) \leq C_{q} \text { and } \sup _{t}\left(\omega_{0}^{-2}\left\{1+\left|\omega_{0} t\right|^{q}\right\} \mid\right. \\
\left.\left\{\partial_{t} \bar{h}_{k}(t)-i \frac{\omega_{k}+\omega_{k-1}}{2} \bar{h}_{k}(t)\right\} \mid\right) \leq C_{q}
\end{gathered}
$$

for some constants depending only on the precise choice of $\bar{\chi}_{3}$. The same bounds also hold for $-n \leq k \leq-1$.

In the same way, we can define the function $\bar{\zeta}_{0}: \mathbb{R} \rightarrow[0,1]$ :

$$
\bar{\zeta}_{0}=\bar{\chi}_{3}\left(\frac{|\omega|}{\omega_{0}}\right)
$$

Then its inverse Fourier transform $\bar{h}_{0}$ also satisfies

$$
\sup _{t}\left(\omega_{0}^{-1}\left\{1+\left|\omega_{0} t\right|^{q}\right\}\left|\bar{h}_{0}(t)\right|\right) \leq C_{q} \text { and } \sup _{t}\left(\omega_{0}^{-2}\left\{1+\left|\omega_{0} t\right|^{q}\right\}\left|\partial_{t} \bar{h}_{k}(t)\right|\right) \leq C_{q} .
$$

Since $\bar{\zeta}_{k} \equiv 1$ on the support of $\zeta_{k}$, we have the relation $\zeta_{k} \cdot \bar{\zeta}_{k}=\zeta_{k}$. This relation implies for $-n \leq k \leq n$ the following self reproducing formula for $\psi_{k}$ :

$$
\psi_{k}(t, \cdot)=\int_{-\infty}^{\infty} \bar{h}_{k}(t-s) \cdot \psi_{k}(s, \cdot) d s
$$

For $1 \leq|k| \leq n$, we can also establish an estimate for the anti-derivative of $\bar{h}_{k}$ : The functions $\tilde{\zeta}_{k}(\omega)=\frac{1}{i \omega} \bar{\zeta}_{k}(\omega)$ are smooth functions of compact support, and in particular they are identically equal to $(i \omega)^{-1}$ on the frequency support of $\hat{\psi}_{k}$ (i.e. the support of $\zeta_{k}$ ). Hence, we have the identity

$$
\tilde{\zeta}_{k}(\omega) \cdot i \omega \hat{\psi}_{k}(\omega, \cdot)=\hat{\psi}_{k}(\omega, \cdot)
$$

Therefore, by denoting with $\tilde{h}_{k}$ the inverse Fourier transform of $\tilde{\zeta}_{k}$, from (4.64) we obtain the relation

$$
\psi_{k}(t, \cdot)=\int_{-\infty}^{\infty} \tilde{h}_{k}(t-s) \cdot T \psi_{k}(s, \cdot) d s
$$


Note also that for any $q \in \mathbb{N}$ we can bound

$$
\begin{aligned}
& \int_{-\infty}^{\infty}\left|\omega_{0}^{q} \frac{d^{q}}{d \omega^{q}} \tilde{\zeta}_{k}(\omega)\right| d \omega \\
& \quad \leq C_{q} \cdot \sum_{l=0}^{q}\left\{\int_{-\infty}^{\infty} \omega_{0}^{q}\left|\frac{d^{l}}{d \omega^{l}}\left(\frac{1}{\omega}\right) \cdot \omega_{0}^{l-q} \cdot \bar{\chi}_{3}^{(q-l)}\left(\frac{\omega-\frac{\omega_{k-1}+\omega_{k}}{2}}{\frac{1}{2}\left(\omega_{k}-\omega_{k-1}\right)}\right)\right| d \omega\right\} \\
& \quad \leq C_{q} \cdot \sum_{l=0}^{q}\left\{\omega_{0}^{l} \int_{-\infty}^{\infty}|\omega|^{-l-1} \cdot\left|\bar{\chi}_{3}^{(q-l)}\left(\frac{\omega-\frac{\omega_{k-1}+\omega_{k}}{2}}{\frac{1}{2}\left(\omega_{k}-\omega_{k-1}\right)}\right)\right| d \omega\right\} \leq \\
& \leq C_{q} \cdot \omega_{k-1}^{-1} \cdot \omega_{0} .
\end{aligned}
$$

However, from the definition of the inverse Fourier transform, for any $q \in \mathbb{N}$ we have

$$
\sup _{t}\left\{\left|\omega_{0} t\right|^{q} \cdot\left|\tilde{h}_{k}(t)\right|\right\} \leq C \cdot \int_{-\infty}^{\infty}\left|\omega_{0}^{q} \frac{d^{q}}{d \omega q} \tilde{\zeta}_{k}(\omega)\right| d \omega
$$

and hence, (4.67) implies:

$$
\sup _{t}\left\{\omega_{0}^{-1}\left\{1+\left|\omega_{0} t\right|^{q}\right\} \cdot\left|\tilde{h}_{k}(t)\right|\right\} \leq C_{q} \cdot \omega_{k-1}^{-1} .
$$

The same statement obviously also holds for $-n \leq k \leq-1$.

We can now establish the following lemma:

Lemma 4.7 For any $1 \leq|k| \leq n$, any $0 \leq t_{1} \leq t_{2} \leq t^{*}$ and any $R \geq 0$, we can bound

$$
\begin{aligned}
& c \cdot \omega_{k-1}^{2}\left\{\int_{\mathcal{R}\left(t_{1}, t_{2}\right) \cap\{r \leq R\}}\left|\psi_{k}\right|^{2}-C\left(\omega_{0}\right) \cdot \int_{t=0} J_{\mu}^{N}(\psi) n^{\mu}\right\} \leq \int_{\mathcal{R}\left(t_{1}, t_{2}\right) \cap\{r \leq R\}}\left|T \psi_{k}\right|^{2} \\
& \leq C \cdot \omega_{k}^{2} \int_{\mathcal{R}\left(t_{1}, t_{2}\right) \cap\{r \leq R\}}\left|\psi_{k}\right|^{2}+\omega_{k}^{2} C\left(\omega_{0}\right) R^{2} \int_{t=0} J_{\mu}^{N}(\psi) n^{\mu},
\end{aligned}
$$

and similarly for $k=0$ :

$$
\int_{\mathcal{R}\left(t_{1}, t_{2}\right) \cap\{r \leq R\}}\left|T \psi_{0}\right|^{2} \leq C \cdot \omega_{0}^{2} \int_{\mathcal{R}\left(t_{1}, t_{2}\right) \cap\{r \leq R\}}\left|\psi_{0}\right|^{2}+C\left(\omega_{0}\right) R^{2} \cdot \int_{t=0} J_{\mu}^{N}(\psi) n^{\mu} .
$$

Remark Notice that the constant multiplying the error term in the right hand side of (4.70) depends on $R$, while this is not the case in the left hand side. Notice also that the constants in front of the $\psi_{k}$ terms in (4.70) do not depend on $\omega_{0}$.

Proof We will just prove the inequality for $1 \leq k \leq n$, since the cases $-n \leq k \leq-1$ and $k=0$ follow in exactly the same way. The proof relies on manipulating the formulas (4.64) and (4.66), together with the bounds (4.61), (4.63) and (4.69). 
By differentiating (4.64) with respect to $t$, we readily deduce as in [21] (using (4.61)) that

$$
\begin{aligned}
\left|\partial_{t} \psi_{k}(t, \cdot)\right| & \leq \int_{-\infty}^{\infty}\left|\partial_{t} \bar{h}_{k}(t-s)\right| \cdot\left|\psi_{k}(s, \cdot)\right| d s \leq \\
& \leq C_{p} \omega_{0}\left(\frac{\omega_{k}+\omega_{k+1}}{2}+\omega_{0}\right) \cdot \int_{-\infty}^{\infty}\left(1+\omega_{0}|t-s|\right)^{-p} \cdot\left|\psi_{k}(s, \cdot)\right| d s \\
& \leq C_{p} \omega_{0} \omega_{k} \int_{-\infty}^{\infty}\left(1+\omega_{0}|t-s|\right)^{-p} \cdot\left|\psi_{k}(s, \cdot)\right| d s
\end{aligned}
$$

Applying a Hölder inequality, and provided that $p>1$ so that $\left(\int_{-\infty}^{\infty}(1+\right.$ $\left.\left.\left|\omega_{0} s\right|\right)^{-p} d\left(\omega_{0} s\right)\right)^{1 / 2} \leq C_{p}<\infty,(4.72)$ yields:

$$
\begin{aligned}
\left|\partial_{t} \psi_{k}(t, \cdot)\right| & \leq C_{p} \omega_{0} \omega_{k} \int_{-\infty}^{\infty}\left(1+\omega_{0}|t-s|\right)^{-p} \cdot\left|\psi_{k}(s, \cdot)\right| d s \\
& \leq C_{p} \omega_{k} \omega_{0}^{1 / 2} \cdot\left(\int_{-\infty}^{\infty}\left(1+\omega_{0}|t-s|\right)^{-p} \cdot\left|\psi_{k}(s, \cdot)\right|^{2} d s\right)^{1 / 2} \\
& \leq C_{p} \omega_{k} \omega_{0}^{1 / 2}\left(\sum_{l=-\infty}^{\infty}(1+|l|)^{-p} \cdot \int_{t+\omega_{0}^{-1} l}^{t+\omega_{0}^{-1}(l+1)}\left|\psi_{k}(s, \cdot)\right|^{2} d s\right)^{1 / 2} .
\end{aligned}
$$

Hence

$$
\begin{aligned}
\int_{t_{1}}^{t_{2}} & \left|\partial_{t} \psi_{k}(t, \cdot)\right|^{2} d t \leq C_{p} \omega_{k}^{2} \omega_{0} \int_{t_{1}}^{t_{2}} \\
& \times\left(\sum_{l=-\infty}^{\infty}(1+|l|)^{-p} \cdot\left(\int_{t+\omega_{0}^{-1} l}^{t+\omega_{0}^{-1}(l+1)}\left|\psi_{k}(s, \cdot)\right|^{2} d s\right)\right) d t \\
\leq & C_{p} \omega_{k}^{2} \omega_{0} \sum_{l=-\infty}^{\infty}(1+|l|)^{-p} \cdot\left(\int_{t_{1}}^{t_{2}} \int_{t+\omega_{0}^{-1} l}^{t+\omega_{0}^{-1}(l+1)}\left|\psi_{k}(s, \cdot)\right|^{2} d s d t\right) \\
\leq & C_{p} \omega_{k}^{2} \omega_{0} \sum_{l=-\infty}^{\infty}(1+|l|)^{-p} \cdot\left(\int_{t_{1}}^{t_{2}} \int_{\omega_{0}^{-1} l}^{\omega_{0}^{-1}(l+1)}\left|\psi_{k}(s+t, \cdot)\right|^{2} d s d t\right) \\
\leq & C_{p} \omega_{k}^{2} \sum_{l=-\infty}^{\infty}(1+|l|)^{-p} \cdot\left(\int_{t_{1}+\omega_{0}^{-1} l}^{t_{2}+\omega_{0}^{-1}(l+1)}\left|\psi_{k}(t, \cdot)\right|^{2} d t\right) \\
\leq & C_{p} \omega_{k}^{2}\left(\int_{t_{1}}^{t_{2}}\left|\Psi_{k}(t, \cdot)\right|^{2} d t\right)+C_{p} \omega_{k}^{2} \sum_{l=-\infty}^{\infty}(1+|l|)^{-p} \\
& \cdot \times\left(\int_{t_{1}+\omega_{0}^{-1} l}^{t_{2}+\omega_{0}^{-1}(l+1)}\left(1-\chi_{\left[t_{1}, t_{2}\right]}\right)\left|\psi_{k}(t, \cdot)\right|^{2} d t\right),
\end{aligned}
$$

where $\chi_{\left[t_{1}, t_{2}\right]}$ is the characteristic function of $\left\{t_{1} \leq t \leq t_{2}\right\}$. 
Integrating (4.74) over $\{r \leq R\}$, we obtain

$$
\begin{aligned}
\int_{\mathcal{R}\left(t_{1}, t_{2}\right) \cap\{r \leq R\}}\left|T \psi_{k}\right|^{2} \leq & C_{p} \omega_{k}^{2} \int_{\mathcal{R}\left(t_{1}, t_{2}\right) \cap\{r \leq R\}}\left|\psi_{k}\right|^{2}+ \\
& +C_{p} \omega_{k}^{2} \sum_{l=-\infty}^{\infty}(1+|l|)^{-p} \\
& \cdot \times\left(\int_{\{r \leq R\}} \int_{t_{1}+\omega_{0}^{-1} l}^{t_{2}+\omega_{0}^{-1}(l+1)}\left(1-\chi_{\left[t_{1}, t_{2}\right]}\right)\left|\psi_{k}(t, x)\right|^{2} d t d x\right) .
\end{aligned}
$$

For the second term of the right hand side of (4.75), we estimate:

$$
\begin{aligned}
& \sum_{l=-\infty}^{-1}(1+|l|)^{-p}\left(\int_{\{r \leq R\}} \int_{t_{1}+\omega_{0}^{-1} l}^{t_{2}+\omega_{0}^{-1}(l+1)}\left(1-\chi_{\left[t_{1}, t_{2}\right]}\right)\left|\psi_{k}(t, x)\right|^{2} d t d x\right) \\
& \leq \sum_{l=-\infty}^{-1}(1+|l|)^{-p}\left(\int_{\{r \leq R\}} \int_{t_{1}+\omega_{0}^{-1} l}^{t_{1}}\left|\psi_{k}(t, x)\right|^{2} d t d x\right) .
\end{aligned}
$$

Notice that for any $q \in \mathbb{N}$ we can bound

$$
\left|\psi_{k}(t, \cdot)\right| \leq \int_{-\infty}^{\infty}\left|h_{k}(t-s)\right| \cdot\left|\psi_{t^{*}}(s, \cdot)\right| d s \leq C_{q} \cdot \omega_{0} \int_{-\infty}^{\infty}\left(1+\omega_{0}|t-s|\right)^{-q} \cdot\left|\psi_{t^{*}}(s, \cdot)\right| d s
$$

due to (4.16), and hence (for $q>1$ )

$$
\left|\psi_{k}(t, \cdot)\right|^{2} \leq C_{q}\left(\omega_{0}\right) \cdot \int_{-\infty}^{\infty}\left(1+\omega_{0}|t-s|\right)^{-q} \cdot\left|\psi_{t^{*}}(s, \cdot)\right|^{2} d s .
$$

Therefore, substituting in (4.76) we infer:

$$
\begin{gathered}
\int_{\{r \leq R\}} \int_{t_{1}+\omega_{0}^{-1} l}^{t_{1}}\left|\Psi_{k}(t, x)\right|^{2} d t d x \leq C_{q}\left(\omega_{0}\right) \int_{t_{1}+\omega_{0}^{-1} l}^{t_{1}} \int_{-\infty}^{\infty}\left(1+\omega_{0}|t-s|\right)^{-q} \\
\cdot\left(\int_{\{r \leq R\} \cap\{t=s\}}\left|\psi_{t^{*}}\right|^{2}\right) d s d t
\end{gathered}
$$

Using a Hardy inequality (such as (3.16)), we can bound:

$$
\begin{aligned}
& \int_{\{t=s\} \cap\{r \leq R\}}\left|\psi_{t^{*}}\right|^{2}=\int_{\{t=s\} \cap\{r \leq R\}}\left|h_{t^{*}}\right|^{2}|\psi|^{2} \leq \int_{\{t=s\} \cap\left\{t_{-} \geq 0\right\} \cap\{r \leq R\}}|\psi|^{2} \\
& \quad \leq C \cdot R^{2} \int_{\{t=s\} \cap\left\{t_{-} \geq 0\right\}} J_{\mu}^{N}(\psi) n^{\mu}
\end{aligned}
$$

for any $s \in \mathbb{R}$. Moreover, as we did in the proofs of the previous lemmas, using the boundedness assumption 4 for $s \geq 0$, and the conservation of the $J^{T}$ current 
and Lemma 4.2 for $s<0$, we can bound for any $s \in \mathbb{R}: \int_{\{t=s\} \cap\left\{t_{-} \geq 0\right\}} J_{\mu}^{N}(\psi) n^{\mu} \leq$ $\int_{t=0} J_{\mu}^{N}(\psi) n^{\mu}$. Thus, inequality (4.80) yields

$$
\int_{\{t=s\} \cap\{r \leq R\}}\left|\psi_{t^{*}}\right|^{2} \leq C \cdot R^{2} \int_{t=0} J_{\mu}^{N}(\psi) n^{\mu} .
$$

Hence, returning to (4.79), we have:

$$
\begin{aligned}
& \int_{\{r \leq R\}} \int_{t_{1}+\omega_{0}^{-1} l}^{t_{1}}\left|\Psi_{k}(t, x)\right|^{2} d t d x \\
& \leq C_{q}\left(\omega_{0}\right) R^{2}\left\{\int_{t_{1}+\omega_{0}^{-1} l}^{t_{1}} \int_{-\infty}^{\infty}\left(1+\omega_{0}(t-s)\right)^{-q} d s d t\right\} \cdot \int_{\{t=0\}} J_{\mu}^{N}(\psi) n^{\mu} \\
& \leq C_{q}\left(\omega_{0}\right) \cdot|l| \cdot R^{2} \int_{\{t=0\}} J_{\mu}^{N}(\psi) n^{\mu}
\end{aligned}
$$

Substituting (4.82) in 4.76, and fixing $p, q$ large enough, we infer the desired bound

$$
\begin{aligned}
& \sum_{l=-\infty}^{-1}(1+|l|)^{-p}\left(\int_{\{r \leq R\}} \int_{t_{1}+\omega_{0}^{-1} l}^{t_{2}+\omega_{0}^{-1}(l+1)}\left(1-\chi_{\left[t_{1}, t_{2}\right]}\right)\left|\psi_{k}(t, x)\right|^{2} d t d x\right) \\
& \leq C\left(\omega_{0}\right) R^{2} \int_{\{t=0\}} J_{\mu}^{N}(\psi) n^{\mu} .
\end{aligned}
$$

In the same way, we can bound

$$
\begin{aligned}
& \sum_{l=0}^{\infty}(1+|l|)^{-p}\left(\int_{\{r \leq R\}} \int_{t_{1}+\omega_{0}^{-1} l}^{t_{2}+\omega_{0}^{-1}(l+1)}\left(1-\chi_{\left[t_{1}, t_{2}\right]}\right)\left|\psi_{k}(t, x)\right|^{2} d t d x\right) \\
& \quad \leq C\left(\omega_{0}\right) R^{2} \int_{\{t=0\}} J_{\mu}^{N}(\psi) n^{\mu} .
\end{aligned}
$$

Returning to (4.75), in view of (4.83) and (4.84) we obtain

$$
\int_{\mathcal{R}\left(t_{1}, t_{2}\right) \cap\{r \leq R\}}\left|T \psi_{k}\right|^{2} \leq C \omega_{k}^{2} \int_{R\left(t_{1}, t_{2}\right) \cap\{r \leq R\}}\left|\psi_{k}\right|^{2}+C\left(\omega_{0}\right) \omega_{k}^{2} \cdot R^{2} \int_{\{t=0\}} J_{\mu}^{N}(\psi) n^{\mu} .
$$

Hence, we have established the right "half" of inequality (4.70).

The left "half" is proved in exactly the same way, but with the use of (4.66) instead of (4.64). That is, in exactly the same way as before (using now (4.69) instead of (4.61)), we obtain

$$
\begin{aligned}
\left|\psi_{k}(t, \cdot)\right| & \leq \int_{-\infty}^{\infty}\left|\tilde{h}_{k}(t-s)\right| \cdot\left|T \psi_{k}(s, \cdot)\right| d s \\
& \leq C_{p} \cdot \omega_{k-1}^{-1} \omega_{0} \cdot \int_{-\infty}^{\infty}\left(1+\omega_{0}|t-s|\right)^{-p} \cdot\left|T \psi_{k}(s, \cdot)\right| d s
\end{aligned}
$$




$$
\leq C_{p} \omega_{k-1}^{-1} \omega_{0}^{1 / 2}\left(\int_{-\infty}^{\infty}\left(1+\omega_{0}|t-s|\right)^{-p} \cdot\left|T \psi_{k}(s, \cdot)\right|^{2} d s\right)^{1 / 2}
$$

and hence (working exactly as before)

$$
\begin{aligned}
& \int_{t_{1}}^{t_{2}}\left|\psi_{k}(t, \cdot)\right|^{2} d t \leq C_{p} \omega_{k-1}^{-2}\left(\int_{t_{1}}^{t_{2}}\left|T \psi_{k}(t, \cdot)\right|^{2} d t\right) \\
& \quad+C_{p} \omega_{k-1}^{-2} \sum_{l=-\infty}^{\infty}(1+|l|)^{-p} \cdot\left(\int_{t_{1}+\omega_{0}^{-1} l}^{t_{2}+\omega_{0}^{-1}(l+1)}\left(1-\chi_{\left[t_{1}, t_{2}\right]}\right)\left|T \psi_{k}(t, \cdot)\right|^{2} d t\right) .
\end{aligned}
$$

After integrating (4.87) over $\{r \leq R\}$, we obtain:

$$
\begin{aligned}
\int_{\mathcal{R}\left(t_{1}, t_{2}\right) \cap\{r \leq R\}}\left|T \psi_{k}\right|^{2} \geq & c_{p} \cdot \omega_{k-1}^{2} \int_{\mathcal{R}\left(t_{1}, t_{2}\right) \cap\{r \leq R\}}\left|\Psi_{k}\right|^{2}-C_{p} \sum_{l=-\infty}^{\infty}(1+|l|)^{-p} \\
& \cdot\left(\int_{r \leq R} \int_{t_{1}+\omega_{0}^{-1} l}^{t_{2}+\omega_{0}^{-1}(l+1)}\left(1-\chi_{\left[t_{1}, t_{2}\right]}\right)\left|T \psi_{k}(t, x)\right|^{2} d t d x\right) .
\end{aligned}
$$

For the last term of the right hand side of (4.88), we work similarly as before: For the part of the infinte sum for $l \leq-1$, we can estimate:

$$
\begin{aligned}
& \sum_{l=-\infty}^{-1}(1+|l|)^{-p}\left(\int_{\{r \leq R\}} \int_{t_{1}+\omega_{0}^{-1} l}^{t_{2}+\omega_{0}^{-1}(l+1)}\left(1-\chi_{\left[t_{1}, t_{2}\right]}\right)\left|T \psi_{k}(t, x)\right|^{2} d t d x\right) \\
& \leq \sum_{l=-\infty}^{-1}(1+|l|)^{-p}\left(\int_{t_{1}+\omega_{0}^{-1} l}^{t_{1}}\left\{\int_{\{t=\tau\}}\left|T \psi_{k}\right|^{2}\right\} d \tau\right) .
\end{aligned}
$$

In order to estimate the second term of the right hand side of (4.89), we proceed as follows: Starting from the bound

$$
\begin{aligned}
& \left|T \psi_{k}(t, \cdot)\right| \leq \int_{-\infty}^{\infty}\left|h_{k}(t-s)\right| \cdot\left|T \psi_{t^{*}}(s, \cdot)\right| d s \\
& \quad \leq C_{q}\left(\omega_{0}\right) \int_{-\infty}^{\infty}\left(1+\omega_{0}|t-s|\right)^{-q} \cdot\left|T \psi_{t^{*}}(s, \cdot)\right| d s
\end{aligned}
$$

for $q>1$, we can control:

$$
\left|T \psi_{k}(t, \cdot)\right|^{2} \leq C_{q}\left(\omega_{0}\right) \int_{-\infty}^{\infty}\left(1+\omega_{0}|t-s|\right)^{-q} \cdot\left|T \psi_{t^{*}}(s, \cdot)\right|^{2} d s .
$$


Thus, we have

$$
\int_{\{t=\tau\}}\left|T \psi_{k}\right|^{2} \leq C_{q}\left(\omega_{0}\right) \int_{-\infty}^{\infty}\left(1+\omega_{0}|\tau-s|\right)^{-q} \cdot\left(\int_{\{t=s\}}\left|T \psi_{t^{*}}\right|^{2}\right) d s .
$$

We can also bound

$$
\left|T \psi_{t^{*}}\right|^{2} \lesssim\left|T h_{t^{*}}\right|^{2}|\psi|^{2}+\left|h_{t^{*}}\right|^{2} \cdot|T \psi|^{2}
$$

Since $T h_{t^{*}}$ is supported in the region $\left\{0 \leq t_{-} \leq 1\right\} \cup\left\{t^{*}-1 \leq t_{+} \leq t^{*}\right\}$, while $h_{t^{*}}$ is supported in $\left\{t_{-} \geq 0\right\}$, we obtain:

$$
\int_{t=s}\left|h_{t^{*}} \cdot T \psi\right|^{2}=\int_{\{t=s\} \cap\left\{t_{-} \geq 0\right\}}|T \psi|^{2} \leq C \cdot \int_{\{t=0\}} J_{\mu}^{N}(\psi) n^{\mu}
$$

for any $s \in \mathbb{R}$. Since $\{t=s\} \cap\left\{0 \leq t_{-} \leq 1\right\} \cup\left\{t^{*}-1 \leq t_{+} \leq t^{*}\right\} \subset\{t=s\} \cap\{r \leq$ $\left.R_{1}+1+2 s\right\}$, we also obtain for any $s \in \mathbb{R}$ through a Hardy inequality:

$$
\begin{aligned}
\int_{\{t=s\}}\left|T h_{t^{*}}\right|^{2}|\psi|^{2} & =\int_{\{t=s\} \cap\left\{0 \leq t_{-} \leq 1\right\} \cup\left\{t^{*}-1 \leq t_{+} \leq t^{*}\right\}}|\psi|^{2} \\
& \leq C \cdot\left(1+\operatorname{dist}\left\{s,\left[0, t^{*}\right]\right\}\right)^{2} \int_{\{t=s\} \cap\left\{t_{-} \geq 0\right\}} J_{\mu}^{N}(\psi) n^{\mu} \\
& \leq C \cdot\left(1+\operatorname{dist}\left\{s,\left[0, t^{*}\right]\right\}\right)^{2} \int_{t=0} J_{\mu}^{N}(\psi) n^{\mu} .
\end{aligned}
$$

Hence, we deduce for $q>3$ from 4.92, (4.94) and (4.95):

$$
\begin{aligned}
& \int_{t_{1}+\omega_{0}^{-1} l}^{t_{1}}\left\{\int_{\{t=\tau\}}\left|T \psi_{k}\right|^{2}\right\} d \tau \leq C_{q}\left(\omega_{0}\right) \\
& \quad\left\{\int_{t_{1}+\omega_{0}^{-1} l}^{t_{1}} \int_{-\infty}^{\infty}\left(1+\omega_{0}|\tau-s|\right)^{-q} \cdot\left(1+\operatorname{dist}\left\{s,\left[0, t^{*}\right]\right\}\right)^{2} d s d \tau\right\} \int_{t=0} J_{\mu}^{N}(\psi) n^{\mu} \\
& \left.\quad \leq C_{q}\left(\omega_{0}\right)\left(1+|l|^{3}\right) \int_{t=0} J_{\mu}^{N}(\psi) n^{\mu}\right\}
\end{aligned}
$$

From 4.89 and (4.96), we conclude for $p, q$ large enough

$$
\begin{aligned}
& \sum_{l=-\infty}^{-1}(1+|l|)^{-p}\left(\int_{\{r \leq R\}} \int_{t_{1}+\omega_{0}^{-1} l}^{t_{2}+\omega_{0}^{-1}(l+1)}\left(1-\chi_{\left[t_{1}, t_{2}\right]}\right)\left|T \psi_{k}(t, x)\right|^{2} d t d x\right) \\
& \leq C\left(\omega_{0}\right) \int_{t=0} J_{\mu}^{N}(\psi) n^{\mu} .
\end{aligned}
$$


Similarly, we can also bound:

$$
\begin{aligned}
& \sum_{l=0}^{\infty}(1+|l|)^{-p}\left(\int_{\{r \leq R\}} \int_{t_{1}+\omega_{0}^{-1} l}^{t_{2}+\omega_{0}^{-1}(l+1)}\left(1-\chi_{\left[t_{1}, t_{2}\right]}\right)\left|T \psi_{k}(t, x)\right|^{2} d t d x\right) \\
& \quad \leq C\left(\omega_{0}\right) \int_{t=0} J_{\mu}^{N}(\psi) n^{\mu} .
\end{aligned}
$$

Hence, we conclude from 4.88, (4.97) and (4.98):

$$
\int_{\mathcal{R}\left(t_{1}, t_{2}\right) \cap\{r \leq R\}}\left|T \psi_{k}\right|^{2} \geq c_{p} \cdot \omega_{k-1}^{2} \int_{\mathcal{R}\left(t_{1}, t_{2}\right) \cap\{r \leq R\}}\left|\psi_{k}\right|^{2}-C\left(\omega_{0}\right) \int_{t=0} J_{\mu}^{N}(\psi) n^{\mu},
$$

thus completing the proof of the Lemma.

We can also establish the following variant of the previous lemma in exactly the same way as before - hence the proof will be omitted:

Lemma 4.8 For any continuous function $\chi: \mathcal{D} \rightarrow[0,+\infty)$ which satisfies the relation $T \mathcal{X}=0$, for any $1 \leq|k| \leq n$ and any $0 \leq t_{1} \leq t_{2} \leq t^{*}, R \geq 0$ we can bound:

$$
\begin{aligned}
c & \cdot \omega_{k-1}^{2}\left\{\int_{\mathcal{R}\left(t_{1}, t_{2}\right) \cap\{r \leq R\}} \chi\left|\psi_{k}\right|^{2}-C\left(\omega_{0}, \chi\right) \cdot \int_{t=0} J_{\mu}^{N}(\psi) n^{\mu}\right\} \\
& \leq \int_{\mathcal{R}\left(t_{1}, t_{2}\right) \cap\{r \leq R\}} \chi\left|T \psi_{k}\right|^{2} \\
& \leq C \cdot \omega_{k}^{2} \int_{\mathcal{R}\left(t_{1}, t_{2}\right) \cap\{r \leq R\}} \chi\left|\psi_{k}\right|^{2}+\omega_{k}^{2} C\left(\omega_{0}, \chi\right) R^{2} \int_{t=0} J_{\mu}^{N}(\psi) n^{\mu},
\end{aligned}
$$

and similarly for $k=0$

$$
\begin{aligned}
\int_{\mathcal{R}\left(t_{1}, t_{2}\right) \cap\{r \leq R\}} \chi\left|T \psi_{0}\right|^{2} \leq & C \cdot \omega_{0}^{2} \int_{\mathcal{R}\left(t_{1}, t_{2}\right) \cap\{r \leq R\}} \chi\left|\psi_{0}\right|^{2} \\
& +C\left(\omega_{0}, \chi\right) R^{2} \cdot \int_{t=0} J_{\mu}^{N}(\psi) n^{\mu} .
\end{aligned}
$$

Finally, we will need the following bounds for the energy of the high frequency part $\psi_{\leq \omega_{+}}$in terms of the initial energy of higher derivatives of $\psi$ :

Lemma 4.9 For any $\tau \in \mathbb{R}$ and any $m \in \mathbb{N}$, there exists a positive constant $C_{m}$ such that

$$
\int_{\{t=\tau\} \cap\left\{r \leq R_{1}\right\}} J_{\mu}^{N}\left(\psi_{\geq \omega_{+}}\right) n^{\mu} \leq \frac{C_{m}}{\omega_{+}^{2 m}} \sum_{j=0}^{m} \int_{t=0} J_{\mu}^{N}\left(T^{j} \psi\right) n^{\mu} .
$$

Proof We can assume without loss of generality that $m \geq 1$, since the $m=0$ case is a direct consequence of Lemma (4.4). 
Recall that we have defined $\chi_{3}: \mathbb{R} \rightarrow[0,1]$ to be a smooth function which is identically 1 on $[-1,1]$ and identically 0 outside of $\left(-\frac{9}{8}, \frac{9}{8}\right)$. We define, in terms of $\chi_{3}$, the function $\chi_{3}^{c} \doteq 1-\chi_{3}$, which vanishes in $[-1,1]$ and is identically 1 outside of $\left(-\frac{9}{8}, \frac{9}{8}\right)$. Then, for any $m \in \mathbb{N}, m \geq 1$, the function $\xi_{m}: \mathbb{R} \rightarrow \mathbb{C}$,

$$
\xi_{m}(y)=\frac{1}{(i y)^{m}} \chi_{3}^{c}(y)
$$

is smooth, vanishing in $[-1,1]$ and equal to $\frac{1}{(i y)^{m}}$ for $|y| \geq \frac{9}{8}$.

We will need the inverse Fourier transform of $\xi_{m}$, namely $\breve{\xi}_{m}(\rho)=\int_{-\infty}^{\infty} e^{i \rho y}$. $\xi_{m}(y) d y$, defined in the sense of tempered distributions. Due to the fact that

$$
\left|\int_{1}^{+\infty} \frac{1}{y} e^{i \lambda} d y\right| \leq C \cdot(|\log (\lambda)|+1)
$$

for any $\lambda>0$, the $\frac{1}{(i y)^{m}}$ asymptotics of $\xi_{m}$ imply that $\check{\xi}_{m}$ is actually a measurable function, satisfying for almost all $y \in \mathbb{R}$ the estimate

$$
\left|\check{\xi}_{m}(\rho)\right| \leq C \cdot(|\log | \rho||+1)
$$

Of course, in the case $m>1$, when $\frac{1}{y^{m}}$ is integrable away from 0 , the $|\log | \rho||$ summand can be removed from (4.105).

Moreover, in view of the fact that $\frac{d}{d y} \chi_{3}^{c}$ is smooth and compactly supported and $\int_{1}^{\infty} \frac{1}{y^{m+1}} d y \leq C$ for any $m \in \mathbb{N}, m \geq 1$, we deduce that $\left|\rho \check{\xi}_{m}(\rho)\right|=\left|\left(\frac{d}{d \rho} \check{\xi}_{m}\right)(\rho)\right| \leq C$ and hence $\left|\check{\xi}_{m}(\rho)\right| \leq \frac{C}{|\rho|}$. Thus, by an induction argument we infer for any $q \in \mathbb{N}$, $q \geq 1$ :

$$
\left|\check{\xi}_{m}(\rho)\right| \leq C_{q, m} \cdot|\rho|^{-q} .
$$

Hence, combining 4.105 and 4.106 we obtain for any $q \in \mathbb{N}, q \geq 1$ :

$$
\left|\check{\xi}_{m}(\rho)\right| \leq C_{q, m} \cdot \frac{|\log | \rho||+1}{1+|\rho|^{q}} .
$$

Due to the fact that $\hat{\psi}_{\geq \omega_{+}}(\cdot, x)$ is supported in $\left\{|\omega| \geq \omega_{+}\right\}$, the following identity holds trivially for all $\omega \in \mathbb{R}$ :

$$
\hat{\psi}_{\geq \omega_{+}}(\omega, \cdot)=\chi_{3}^{c}\left(\frac{2 \omega}{\omega_{+}}\right) \cdot \hat{\psi}_{\geq \omega_{+}}(\omega, \cdot) .
$$

In view of the definition (4.103) of $\xi_{m}$, we infer that for any $m \in \mathbb{N}, m \geq 1$ :

$$
\hat{\psi}_{\geq \omega_{+}}(\omega, \cdot)=\left(\frac{\omega_{+}}{2}\right)^{-m} \cdot \xi_{m}\left(\frac{2 \omega}{\omega_{+}}\right) \cdot T^{\hat{m}} \psi_{\geq \omega_{+}}(\omega, \cdot) .
$$


Thus, applying the inverse Fourier transform we obtain:

$$
\psi_{\geq \omega_{+}}(t, \cdot)=2^{m-1} \omega_{+}^{-m+1} \cdot \int_{-\infty}^{\infty} \check{\xi}_{m}\left(\frac{1}{2} \omega_{+}(t-s)\right) \cdot T^{m} \psi_{\geq \omega_{+}}(s, \cdot) d s .
$$

This formula is also valid for the derivatives of $\psi_{\geq \omega_{+}}$:

$$
d \psi_{\geq \omega_{+}}(t, \cdot)=2^{m-1} \omega_{+}^{-m+1} \cdot \int_{-\infty}^{\infty} \check{\xi}_{m}\left(\frac{1}{2} \omega_{+}(t-s)\right) \cdot d\left(T^{m} \psi_{\geq \omega_{+}}\right)(s, \cdot) d s .
$$

In view of the fact that $\int_{-\infty}^{\infty}\left|\check{\xi}_{m}\left(\frac{1}{2} \omega_{+} t\right)\right| d t \leq C_{m} \cdot \omega_{+}^{-1}$, we compute from (4.111) after contracting with $T$ that:

$$
\begin{aligned}
\left|T \psi_{\geq \omega_{+}}(t, \cdot)\right|^{2}=2^{2 m-2} \omega_{+}^{-2 m+2} \cdot\left|\int_{-\infty}^{\infty} \check{\xi}_{m}\left(\frac{1}{2} \omega_{+}(t-s)\right) \cdot T^{m+1} \Psi_{\geq \omega_{+}}(s, \cdot) d s\right|^{2} \\
\leq 2^{2 m-2} \omega_{+}^{-2 m+2} \cdot\left\{\int_{-\infty}^{\infty}\left|\check{\xi}_{m}\left(\frac{1}{2} \omega_{+}(t-s)\right)\right| d s\right\} \\
\quad \cdot\left\{\int_{-\infty}^{\infty}\left|\check{\xi}_{m}\left(\frac{1}{2} \omega_{+}(t-s)\right)\right| \cdot\left|T^{m+1} \psi_{\geq \omega_{+}}(s, \cdot)\right|^{2} d s\right\} \\
\leq C_{m} \cdot \omega_{+}^{-2 m+1} \cdot\left\{\int_{-\infty}^{\infty}\left|\check{\xi}_{m}\left(\frac{1}{2} \omega_{+}(t-s)\right)\right| \cdot\left|T^{m+1} \Psi_{\geq \omega_{+}}(s, \cdot)\right|^{2} d s\right\}
\end{aligned}
$$

and, after applying (4.107), we obtain:

$$
\begin{aligned}
& \left|T \psi_{\geq \omega_{+}}(t, \cdot)\right|^{2} \leq C_{m, q} \cdot \omega_{+}^{-2 m+1} \\
& \quad \cdot\left\{\int_{-\infty}^{\infty}\left|\frac{\left|\log \left(\omega_{+}(t-s)\right)\right|+1}{1+\left|\omega_{+}(t-s)\right|^{q}}\right| \cdot\left|T^{m+1} \psi_{\geq \omega_{+}}(s, \cdot)\right|^{2} d s\right\} .
\end{aligned}
$$

Hence, for $0 \leq \tau \leq t^{*}$ we can bound

$$
\begin{aligned}
& \int_{\{t=\tau\} \cap\left\{r \leq R_{1}\right\}}\left|T \psi_{\geq \omega_{+}}\right|^{2} \leq C_{m, q} \cdot \omega_{+}^{-2 m+1} \\
& \quad \cdot\left\{\int_{-\infty}^{\infty}\left|\frac{\left|\log \left(\omega_{+}(\tau-s)\right)\right|+1}{1+\left|\omega_{+}(\tau-s)\right|^{q}}\right| \cdot\left\{\int_{\{t=s\} \cap\left\{r \leq R_{1}\right\}}\left|T^{m+1} \psi_{\geq \omega_{+}}\right|^{2}\right\} d s\right\} .
\end{aligned}
$$

Using (4.15) for some $q^{\prime}>1$ and the fact that $\psi_{\geq \omega_{+}}(t, \cdot)=\int_{-\infty}^{\infty} h_{\geq \omega_{+}}(t-$ $s) \psi_{t^{*}}(s) d s$, we compute after an application of Hölder's inequality that

$$
\left|T^{m+1} \psi_{\geq \omega_{+}}(t, \cdot)\right|^{2} \leq C_{m, q^{\prime}} \cdot \omega_{+} \int_{-\infty}^{\infty} \frac{1}{1+\left|\omega_{+}(t-s)\right|^{q^{\prime}}} \cdot\left|T^{m+1} \psi_{t^{*}}(s, \cdot)\right|^{2} d s,
$$


which after an integration over $\left\{r \leq R_{1}\right\}$ gives us for any $\tau \in \mathbb{R}$ :

$$
\begin{aligned}
& \int_{\{t=\tau\} \cap\left\{r \leq R_{1}\right\}}\left|T^{m+1} \psi_{\geq \omega_{+}}\right|^{2} \leq C_{m, q^{\prime}} \cdot \omega_{+} \int_{-\infty}^{\infty} \frac{1}{1+\left|\omega_{+}(\tau-s)\right|^{q^{\prime}}} \\
& \quad \cdot\left\{\int_{\{t=s\} \cap\left\{r \leq R_{1}\right\}}\left|T^{m+1} \psi_{t^{*}}\right|^{2}\right\} d s .
\end{aligned}
$$

Similarly, repeating the same procedure after contracting (4.111) with $T$ invariant vector fields tangential to the foliations $\Sigma_{\tau}$, we can also bound

$$
\begin{aligned}
& \int_{\{t=\tau\} \cap\left\{r \leq R_{1}\right\}}\left|\nabla_{\Sigma}\left(T^{m} \psi_{\geq \omega_{+}}\right)\right|_{g_{\Sigma}}^{2} \leq C_{m, q^{\prime}} \cdot \omega_{+} \int_{-\infty}^{\infty} \frac{1}{1+\left|\omega_{+}(\tau-s)\right|^{q^{\prime}}} \\
& \quad\left\{\int_{\{t=s\} \cap\left\{r \leq R_{1}\right\}}\left|\nabla_{\Sigma}\left(T^{m} \psi_{t^{*}}\right)\right|_{g_{\Sigma}}^{2}\right\} d s .
\end{aligned}
$$

Recall that $\psi_{t^{*}}=h_{t^{*}} \cdot \psi$, and in $\left\{r \leq R_{1}\right\} h_{t^{*}}$ is non zero only for $\left\{0 \leq t \leq t^{*}\right\}$. Thus, (4.116) and (4.117) for $q^{\prime}=2$ yield, in view of the Hardy inequality

$$
\int_{\{t=s\} \cap\left\{r \leq R_{1}\right\}}|\psi|^{2} \leq C\left(R_{1}\right) \cdot \int_{\{t=s\}} J_{\mu}^{N}(\psi) n^{\mu}
$$

and the boundedness assumption 4 , that

$$
\int_{\{t=\tau\} \cap\left\{r \leq R_{1}\right\}} J_{\mu}^{N}\left(T^{m} \psi_{\geq \omega_{+}}\right) n^{\mu} \leq C_{m} \sum_{j=0}^{m} \int_{t=0} J_{\mu}^{N}\left(T^{j} \psi\right) n^{\mu} .
$$

Using (4.119) and (4.114) for $q=2$ we infer the desired inequality for any $\tau \in \mathbb{R}$ :

$$
\int_{\{t=\tau\} \cap\left\{r \leq R_{1}\right\}} J_{\mu}^{N}\left(\Psi_{\geq \omega_{+}}\right) n^{\mu} \leq \frac{C_{m}}{\omega_{+}^{2 m}} \sum_{j=0}^{m} \int_{t=0} J_{\mu}^{N}\left(T^{j} \psi\right) n^{\mu} .
$$

\section{Estimates for $\psi_{k}$ in the Asymptotically Flat Region}

In this section, we will specialise some of the estimates established in [52] for general asymptotically flat spacetimes to our setting. As we did in Section (4), we will assume that we are given a smooth function $\psi: \mathcal{D} \rightarrow \mathbb{C}$ solving $\square_{g} \psi=0$ on $J^{+}(\Sigma) \cap \mathcal{D}$ with compactly supported initial data on $\Sigma$, together with a set of parameters $t^{*}, \omega_{0}, \omega_{+}$, leading to the construction of the functions $\psi_{t^{*}}, \psi_{\leq \omega_{+}}, \psi_{\geq \omega_{+}}$and $\psi_{k}$ (as performed in Section 4). We will derive estimates for the functions $\psi_{k}, \psi_{\leq \omega_{+}}$and $\psi_{\geq \omega_{+}}$in the asymptotically flat region $\{r \gg 1\}$ of $\mathcal{D}$

We will make use of the fact that there exists a function $u$ in the region $\{r \gg 1\}$ (see the Appendix) such that in the $(u, r, \sigma)$ coordinate system the metric $g$ has the form: 


$$
\begin{aligned}
g= & -4\left(1-\frac{2 M}{r}+O_{3}\left(r^{-1-a}\right)\right) d u^{2}-4\left(1+O_{3}\left(r^{-1-a}\right)\right) d u d r+r^{2}\left(g_{\mathbb{S}^{d-1}}\right. \\
& \left.+O_{3}\left(r^{-1-a}\right)\right)+O_{3}\left(r^{-a}\right) d u d \sigma+O_{3}\left(r^{-a}\right) d r d \sigma .
\end{aligned}
$$

We will also introduce the function $v=u+r$, noting that in the $(u, v, \sigma)$ coordinate chart the metric $g$ takes the form:

$$
\begin{aligned}
g= & -\left(4+O_{3}\left(r^{-1-a}\right)\right) d v d u+r^{2} \cdot\left(g_{\mathbb{S}^{d-1}}+O_{3}\left(r^{-1-a}\right)\right)+O_{3}\left(r^{-a}\right) d u d \sigma \\
& +O_{3}\left(r^{-a}\right) d v d \sigma+4\left(-\frac{2 M}{r}+O_{3}\left(r^{-1-a}\right)\right) d u^{2}
\end{aligned}
$$

Let us remark that most of the results of [52] were stated under the requirement that the metric $g$ in the asymptotic region is of the form

$$
\begin{aligned}
g= & -\left(4+O_{m}\left(r^{-1-a}\right)\right) d v d u+r^{2} \cdot\left(g_{\mathbb{S} d-1}+O_{m}\left(r^{-1-a}\right)\right)+O_{m}\left(r^{-a}\right) d u d \sigma \\
& +O_{m}\left(r^{-a}\right) d v d \sigma+4\left(-\frac{2 M}{r}+O_{m}\left(r^{-1-a}\right)\right) d u^{2}
\end{aligned}
$$

for some large enough integer $m$, i.e. it was assumed in [52] that $g$ is smoother on $\mathcal{I}^{+}$than what is assumed in the present paper (actually, in [52] the metrics considered where of more general form than (5.3), but this fact is not relevant for the present paper).However, the results of [52] that we will make use of in this section can be established also for a metric with the rougher asymptotics (5.1) (as can be readily verified by an inspection of their proof).

\subsection{Some $\partial_{r}$-Morawetz Type Estimates}

We will establish the following Lemma:

Lemma 5.1 For any given $0<\eta<a$, there exists an $R=R(\eta)>0$ and $C(\eta), C\left(\omega_{0}, \eta\right)>0$ such that for any $-n \leq k \leq n$ and any smooth cut-off function $\chi: \mathcal{D} \rightarrow[0,1]$ supported in $\{r \geq R\}$, we can bound:

$$
\begin{aligned}
& \int_{\mathcal{R}\left(0, t^{*}\right)} \chi \cdot\left(r^{-1-\eta}\left(\left|\partial_{t} \psi_{k}\right|^{2}+\left|\partial_{r} \psi_{k}\right|^{2}\right)+r^{-1}\left|r^{-1} \partial_{\sigma} \psi_{k}\right|^{2}+r^{-3-\eta}\left|\psi_{k}\right|^{2}\right) \\
& \leq C(\eta) \cdot \int_{\{\operatorname{supp}(\partial \chi)\} \cap \mathcal{R}\left(0, t^{*}\right)}|\partial \chi| \cdot J_{\mu}^{N}\left(\psi_{k}\right) n^{\mu}+C\left(\omega_{0}, \eta\right) \cdot \int_{t=0} J_{\mu}^{N}(\psi) n^{\mu} .
\end{aligned}
$$

The same estimate holds for $\psi_{\leq \omega_{+}}, \psi_{\geq \omega_{+}}$in place of $\psi_{k}$.

Remark We have used the notation $|\partial h|^{2}=\left|\partial_{t} h\right|^{2}+\left|\partial_{r} h\right|^{2}+\left|\frac{1}{r} \partial_{\sigma} h\right|^{2}$. 
Proof From Lemma 4.2 of [52], we can bound for any smooth function $\omega: \mathcal{D} \rightarrow \mathbb{C}$ with compact support in space (in the $(u, v, \sigma)$ coordinate system constructed in the Appendix on the region $\{r \gg 1\})$ :

$$
\begin{aligned}
& \int_{\mathcal{R}\left(0, t^{*}\right)} \chi \cdot\left(r^{-1-\eta}\left(\left|\partial_{u} \omega\right|^{2}+\left|\partial_{v} \omega\right|^{2}\right)+r^{-1}\left|r^{-1} \partial_{\sigma} \omega\right|^{2}+r^{-3-\eta}|\omega|^{2}\right) \\
& \leq C_{\eta} \int_{\mathcal{R}\left(0, t^{*}\right)}|\partial \chi| \cdot\left(|\partial \omega|^{2}+r^{-2}|\omega|^{2}\right) \\
& \quad+C_{\eta} \int_{\{t=0\} \cap\{r \geq R\}} J_{\mu}^{T}(\omega) n^{\mu}+C_{\eta} \int_{\left\{t=t^{*}\right\} \cap\{r \geq R\}} J_{\mu}^{T}(\omega) n^{\mu} \\
& \quad+\int_{\mathcal{R}\left(0, t^{*}\right)} \chi \cdot \operatorname{Re}\left\{\left(O_{\eta}(1)\left(\partial_{v}-\partial_{u}\right) \bar{\omega}+O_{\eta}\left(r^{-1}\right) \bar{\omega}\right) \cdot \square_{g} \omega\right\} .
\end{aligned}
$$

In view of Lemmas (4.3) and (4.4) (as well as the properties of the functions $u, v$ ), inequality (5.4) follows readily from (5.5) after substituting $\psi_{k}$ (or $\psi_{\leq \omega_{+}}, \psi_{\geq \omega_{+}}$ respectively) in place of $\omega$ (and using a Cauchy-Schwarz inequality for the last term of the right hand side). ${ }^{22}$

We can also present the previous estimate expressed in a more refined form in terms of the "boundary" terms of the right hand side:

Lemma 5.2 For any given $0<\eta<a$ and any $R>0$ large enough in terms of $\eta$, there exist $C(\eta), C\left(\omega_{0}, \eta\right)>0$ such that for any $-n \leq k \leq n$ we can bound:

$$
\begin{aligned}
& \int_{\{r \geq 2 R\} \cap \mathcal{R}\left(0, t^{*}\right)}\left(r^{-1-\eta}\left(\left|\partial_{t} \psi_{k}\right|^{2}+\left|\partial_{r} \psi_{k}\right|^{2}\right)+r^{-1}\left|r^{-1} \partial_{\sigma} \psi_{k}\right|^{2}+r^{-3-\eta}\left|\psi_{k}\right|^{2}\right) \\
& \leq C(\eta) \cdot \int_{\{R \leq r \leq 2 R\} \cap \mathcal{R}\left(0, t^{*}\right)}\left(R^{-1} J_{\mu}^{N}\left(\psi_{k}\right) n^{\mu}+R^{-3}\left|\psi_{k}\right|^{2}\right) \\
& \quad+C\left(\omega_{0}, \eta\right) \int_{t=0} J_{\mu}^{N}(\psi) n^{\mu}
\end{aligned}
$$

The same estimate also holds for $\psi_{\leq \omega_{+}}, \psi_{\geq \omega_{+}}$in place of $\psi_{k}$.

Proof Inequality (5.6) can be proven by fixing a smooth cut-off $\xi:[0,+\infty) \rightarrow[0,1]$ such that $\xi \equiv 0$ for $r \leq 1$ and $\xi \equiv 1$ for $r \geq 2$, and defining $\chi=\chi_{R}: D \rightarrow$ $[0,1], \chi_{R}=\xi \circ\left(\frac{r}{R}\right)$ for any $R>0$. We easily calculate that there exists a constant $C>0$ depending on the precise choice of $\xi$, such that $\left|\partial \chi_{R}\right| \leq C \cdot R^{-1}$. Moreover, $\operatorname{supp}\left(\partial \chi_{R}\right) \subseteq\{R \leq r \leq 2 R\}$. The result then follows by using $\chi_{R}$ as a cut-off function in the statement of the previous lemma (5.1), for any $R>0$ sufficiently large in terms of $\eta$.

We will also need a variant of the previous lemma, that provides improved control of the spacetime integral of the energy of $\psi_{k}, \psi_{\leq \omega_{+}}, \psi_{\geq \omega_{+}}$over compact subsets. We

\footnotetext{
22 Notice that here we have used the fact that the functions $\psi_{k}, \psi_{\leq \omega_{+}}, \psi_{\geq \omega_{+}}$have compact support in space, since they are supported in a cylinder $\left\{r \lesssim R_{\text {sup }}+t^{*}\right\}$.
} 
we will only need to use it in the case of $\psi_{0}$, but we can state it for all the components $\psi_{k}, \psi_{\leq \omega_{+}}, \psi_{\geq \omega_{+}}$of $\psi$ :

Lemma 5.3 For any given $0<\eta<a$, any $R>0$ sufficiently large in terms of $\eta$, there exists some $C(\eta)>0$ such that for any $R_{c} \geq 2 R$ and any $-n \leq k \leq n$ we can bound

$$
\begin{aligned}
& \int_{\left\{2 R \leq r \leq R_{c}\right\} \cap \mathcal{R}\left(0, t^{*}\right)}\left(r^{-\eta}\left(\left|\partial_{t} \psi_{k}\right|^{2}+\left|\partial_{r} \psi_{k}\right|^{2}+\left|r^{-1} \partial_{\sigma} \psi_{k}\right|^{2}\right)+r^{-2-\eta}\left|\psi_{k}\right|^{2}\right) \\
& \leq C(\eta) \cdot \int_{\{R \leq r \leq 2 R\} \cap \mathcal{R}\left(0, t^{*}\right)}\left(J_{\mu}^{N}\left(\psi_{k}\right) n^{\mu}+R^{-2}\left|\psi_{k}\right|^{2}\right) \\
& \quad+C\left(\omega_{0}, R_{c}, \eta\right) \int_{t=0} J_{\mu}^{N}(\psi) n^{\mu} .
\end{aligned}
$$

The same estimate also holds for $\psi_{\leq \omega_{+}}, \psi_{\geq \omega_{+}}$in place of $\psi_{k}$.

Remark Notice that the constant in front of the $\{R \leq r \leq 2 R\}$ terms does not depend on $R_{c}$.

Proof This is an immediate corollary of Lemma 4.4 of [52] for $\psi_{k}$ in place of $\omega$, which yields

$$
\begin{aligned}
& \int_{\mathcal{R}\left(0, t^{*}\right) \cap\left\{r \leq R_{c}\right\}} \chi \cdot\left(\left(\left|\partial_{u} \psi_{k}\right|^{2}+\left|\partial_{v} \psi_{k}\right|^{2}\right)+\left|r^{-1} \partial_{\sigma} \psi_{k}\right|^{2}+r^{-2}\left|\psi_{k}\right|^{2}\right) \\
& \leq C(\eta) \cdot \int_{\mathcal{R}\left(0, t^{*}\right)}|\partial \chi| \cdot r \cdot\left(\left|\partial \psi_{k}\right|^{2}+r^{-2}\left|\psi_{k}\right|^{2}\right) \\
& \quad+C\left(\eta, R_{c}\right) \cdot \int_{\{t=0\} \cap\{r \geq R\}}\left|\partial \psi_{k}\right|^{2}+C\left(\eta, R_{c}\right) \cdot \int_{\left\{t=t^{*}\right\} \cap\{r \geq R\}}\left|\partial \psi_{k}\right|^{2} \\
& \quad+\int_{\mathcal{R}\left(0, t^{*}\right)} \chi \cdot \operatorname{Re}\left\{\left(O_{R_{c}, \eta}(1)\left(\partial_{v}-\partial_{u}\right) \bar{\psi}_{k}+O_{R_{c}, \eta}\left(r^{-1}\right) \bar{\psi}_{k}\right) \cdot \square_{g} \psi_{k}\right\},
\end{aligned}
$$

combined with Lemmas 4.3 and 4.4. Similarly for $\psi_{\leq \omega_{+}}, \psi_{\geq \omega_{+}}$.

\subsection{Some $r^{p}$-Weighted Energy Estimates}

The following $r^{p}$-weighted energy estimate can be established as a Corollary of Theorem 5.3 of [52] (notice that $(v, \sigma)$ defines a regular polar coordinate map in the region $\{r \gg 1\}$ of the hypersurfaces $\{t=$ const $\})$ :

Lemma 5.4 For any $0<p \leq 2$, any $0<\eta<a$, any $0<\delta<1$ and any $R>0$ large enough in terms of $p, \eta, \delta$, the following inequality holds for any $-n \leq k \leq n$, any $0 \leq t_{1} \leq t_{2} \leq t^{*}$ and any smooth cut-off $\chi_{R}: \mathcal{D} \rightarrow[0,1]$ supported in $\{r \geq R\}$ so that $\chi_{R} \equiv 1$ on $\{r \geq 2 R\}$ : (we also set $\Psi_{k} \doteq r^{\frac{d-1}{2}} \cdot \psi_{k}$ )

$$
\int_{\left\{t=t_{2}\right\}} \chi_{R} \cdot\left(r^{p}\left|\partial_{v} \Psi_{k}\right|^{2}+r^{p}\left|r^{-1} \partial_{\sigma} \Psi_{k}\right|^{2}+\left((d-3) r^{p-2}+\min \left\{r^{p-2}, r^{-\delta}\right\}\right)\left|\Psi_{k}\right|^{2}\right) d v d \sigma
$$




$$
\begin{aligned}
& +\int_{\mathcal{R}\left(t_{1}, t_{2}\right)} \chi_{R} \cdot\left(p r^{p-1}\left|\partial_{v} \Psi_{k}\right|^{2}+\left\{\left((2-p) r^{p-1}+r^{p-1-\delta}\right)\left|r^{-1} \partial_{\sigma} \Psi_{k}\right|^{2}\right.\right. \\
& \left.\left.+\left((2-p)(d-3) r^{p-3}+\min \left\{r^{p-3}, r^{-1-\delta}\right\}\right)\left|\Psi_{k}\right|^{2}\right\}+r^{-1-\eta}\left|\partial_{u} \Psi_{k}\right|^{2}\right) d u d v d \sigma \\
& \leq C(p, \eta, \delta) \cdot \int_{\mathcal{R}\left(t_{1}, t_{2}\right)}\left|\partial \chi_{R}\right| \cdot\left(r^{p}\left|\partial \Psi_{k}\right|^{2}+r^{p-2}\left|\Psi_{k}\right|^{2}\right) d u d v d \sigma \\
& +C\left(\omega_{0}, p, \eta, R, \chi_{R}, \delta\right) \cdot \int_{\{t=0\}}\left(1+r^{p}\right) \cdot J_{\mu}^{N}(\psi) n^{\mu} .
\end{aligned}
$$

The same estimate also holds for $\psi_{\leq \omega_{+}}, \psi_{\geq \omega_{+}}$in place of $\psi_{k}$.

Proof We will establish the result only for $\psi_{k}$, since the proof for the cases of $\psi_{\leq \omega_{+}}, \psi_{\geq \omega_{+}}$is identical.

We will set

$$
\Psi_{k} \doteq r^{\frac{d-1}{2}} \psi_{k}
$$

In view of Theorem 5.3 of [52] (with $\psi_{k}$ in place of $\omega$ ), using also of the fact that in the region $\{r \gg 1\}$ in the $(u, v, \sigma)$ coordinate system we have the relation

$$
\operatorname{det}(g)=-4 r^{2(d-1)}\left(1+O_{3}\left(r^{-1}\right)\right)
$$

we can bound:

$$
\begin{aligned}
\int_{\left\{t=t_{2}\right\}} \chi_{R} \cdot\left(r^{p}\left|\partial_{v} \Psi_{k}\right|^{2}+r^{p}\left|r^{-1} \partial_{\sigma} \Psi_{k}\right|^{2}+\left((d-3) r^{p-2}+\min \left\{r^{p-2}, r^{-\delta}\right\}\right)\left|\Psi_{k}\right|^{2}\right) d v d \sigma \\
\quad+\int_{\mathcal{R}\left(t_{1}, t_{2}\right)} \chi_{R} \cdot\left(p r^{p-1}\left|\partial_{v} \Psi_{k}\right|^{2}+\left\{\left((2-p) r^{p-1}+r^{p-1-\delta}\right)\left|r^{-1} \partial_{\sigma} \Psi_{k}\right|^{2}\right.\right. \\
\left.\left.\quad+\left((2-p)(d-3) r^{p-3}+\min \left\{r^{p-3}, r^{-1-\delta}\right\}\right)\left|\Psi_{k}\right|^{2}\right\}+r^{-1-\eta}\left|\partial_{u} \Psi_{k}\right|^{2}\right) d u d v d \sigma \lesssim p, \delta \\
\lesssim_{p, \eta, \delta} \int_{\left\{t=t_{1}\right\}} \chi_{R} \cdot\left(r^{p}\left|\partial_{v} \Psi_{k}\right|^{2}+r^{p}\left|r^{-1} \partial_{\sigma} \Psi_{k}\right|^{2}+\left((d-3) r^{p-2}+\min \left\{r^{p-2}, r^{-\delta}\right\}\right)\left|\Psi_{k}\right|^{2}\right) d v d \sigma \\
\quad+\int_{\left\{t=t_{1}\right\}} \chi_{R} J_{\mu}^{T}\left(\Psi_{k}\right) n^{\mu}+\int_{\mathcal{R}\left(t_{1}, t_{2}\right)}\left|\partial \chi_{R}\right| \cdot\left(r^{p}\left|\partial \Psi_{k}\right|^{2}+r^{p-2} \cdot\left|\Psi_{k}\right|^{2}\right) \\
\quad+\int_{\mathcal{R}\left(t_{1}, t_{2}\right)} \chi_{R} \cdot\left(r^{p+1}+r^{1+\eta}\right) \cdot\left|F_{k}\right|^{2} \Omega^{2} d u d v d \sigma .
\end{aligned}
$$

Thus, in view also of Lemmas 4.3 and 4.4, in order to reach (5.9) it suffices to prove that

$$
\begin{aligned}
& \int_{\left\{t=t_{1}\right\}} \chi_{R} \cdot\left(r^{p}\left|\partial_{v} \Psi_{k}\right|^{2}+r^{p}\left|r^{-1} \partial_{\sigma} \Psi_{k}\right|^{2}+\left((d-3) r^{p-2}\right.\right. \\
& \left.\left.\quad+\min \left\{r^{p-2}, r^{-\delta}\right\}\right)\left|\Psi_{k}\right|^{2}\right) d v d \sigma \\
& \quad \leq C\left(\omega_{0}, R, p, \chi_{R}\right) \cdot \int_{\{t=0\}}\left(1+r^{p}\right) \cdot J_{\mu}^{N}(\psi) n^{\mu}
\end{aligned}
$$

Inequality (5.13) will be established in the way that Lemma 4.4 was proven, the only difference being that instead of the boundedness assumption 4 for $\psi$, we will mainly use the following estimate for $\psi$ (obtained by applying Theorem 5.3 of [52], 
after repeating the proof in the region $\{t \leq s\} \cap\left\{t_{-} \geq 0\right\}$ for any $s \in \mathbb{R}$ ): setting $\Psi=r^{\frac{d-1}{2}} \psi$, we can bound for any $s \in \mathbb{R}$

$$
\begin{aligned}
\int_{\{t=s\} \cap\left\{t_{-} \geq 0\right\}} \chi_{R} \cdot\left(r^{p}\left|\partial_{v} \Psi\right|^{2}+r^{p}\left|r^{-1} \partial_{\sigma} \Psi\right|^{2}+\left((d-3) r^{p-2}\right.\right. \\
\left.\left.\quad+\min \left\{r^{p-2}, r^{-\delta}\right\}\right)|\Psi|^{2}\right) d v d \sigma \\
\quad+\int_{\left\{t_{-} \geq 0\right\} \cap\{t \leq s\}} \chi_{R} \cdot\left(p r^{p-1}\left|\partial_{v} \Psi\right|^{2}+\left\{\left((2-p) r^{p-1}+r^{p-1-\delta}\right)\left|r^{-1} \partial_{\sigma} \Psi\right|^{2}\right.\right. \\
\left.\left.\quad+\left((2-p)(d-3) r^{p-3}+\min \left\{r^{p-3}, r^{-1-\delta}\right\}\right)|\Psi|^{2}\right\}+r^{-1-\eta}\left|\partial_{u} \Psi\right|^{2}\right) d u d v d \sigma \lesssim p, \delta \\
\lesssim p, \eta, \delta \int_{\left\{t_{-}=0\right\} \cap\{t \leq s\}} \chi_{R} \cdot\left(r^{p}\left|\partial_{v} \Psi\right|^{2}+r^{p}\left|r^{-1} \partial_{\sigma} \Psi\right|^{2}\right. \\
\left.\quad+\left((d-3) r^{p-2}+\min \left\{r^{p-2}, r^{-\delta}\right\}\right)|\Psi|^{2}\right) d v d \sigma+\int_{\left\{t_{-}=0\right\} \cap\{t \leq s\}} \chi_{R} J_{\mu}^{T}(\Psi) n^{\mu} \\
\quad+\int_{\left\{t_{-} \geq 0\right\} \cap\{t \leq s\}}\left|\partial \chi_{R}\right| \cdot\left(r^{p}|\partial \psi|^{2}+r^{p-2} \cdot|\psi|^{2}\right) .
\end{aligned}
$$

Using the boundedness assumption 4 for $s \geq 0$, and the conservation of the $J^{T}$ current in the region $\left\{t_{-} \geq 0\right\} \cap\{t \leq s\}$ for $s<0$, as well as Lemma 4.2, we can bound

$$
\int_{\left\{t_{-}=0\right\} \cap\{t \leq s\}} J_{\mu}^{N}(\psi) n^{\mu} \leq C \cdot \int_{\{t=0\}} J_{\mu}^{N}(\psi) n^{\mu} .
$$

Using Lemma 4.2 and a Hardy inequality

$$
\int_{\left\{t_{-}=0\right\} \cap\{t \leq s\}} r^{p-2}|\psi|^{2} \leq C(p) \cdot \int_{\left\{t_{-}=0\right\} \cap\{t \leq s\}} r^{p} J_{\mu}^{N}(\psi) n^{\mu}
$$

we can also bound:

$$
\begin{aligned}
& \int_{\left\{t_{-}=0\right\} \cap\{t \leq s\}} \chi_{R} \\
& \quad \cdot\left(r^{p}\left|\partial_{v} \Psi\right|^{2}+r^{p}\left|r^{-1} \partial_{\sigma} \Psi\right|^{2}+\left((d-3) r^{p-2}+\min \left\{r^{p-2}, r^{-\delta}\right\}\right)|\Psi|^{2}\right) d v d \sigma \\
& \leq C(p) \cdot \int_{t=0}\left(1+r^{p}\right) \cdot J_{\mu}^{N}(\psi) n^{\mu}
\end{aligned}
$$

Therefore, returning to (5.14) we obtain:

$$
\begin{aligned}
\int_{\{t=s\} \cap\left\{t_{-} \geq 0\right\}} \chi_{R} \cdot\left(r^{p}\left|\partial_{v} \Psi\right|^{2}\right. \\
\left.+r^{p}\left|r^{-1} \partial_{\sigma} \Psi\right|^{2}+\left((d-3) r^{p-2}+\min \left\{r^{p-2}, r^{-\delta}\right\}\right)|\Psi|^{2}\right) d v d \sigma \\
\quad+\int_{\left\{t_{-} \geq 0\right\} \cap\{t \leq s\}} \chi_{R} \cdot\left(p r^{p-1}\left|\partial_{v} \Psi\right|^{2}+\left\{\left((2-p) r^{p-1}+r^{p-1-\delta}\right)\left|r^{-1} \partial_{\sigma} \Psi\right|^{2}\right.\right.
\end{aligned}
$$




$$
\begin{aligned}
& \left.\left.+\left((2-p)(d-3) r^{p-3}+\min \left\{r^{p-3}, r^{-1-\delta}\right\}\right)|\Psi|^{2}\right\}+r^{-1-\eta}\left|\partial_{u} \Psi\right|^{2}\right) d u d v d \sigma \lesssim p, \delta \\
\lesssim p, \eta, \delta & \int_{t=0}\left(1+r^{p}\right) \cdot J_{\mu}^{N}(\psi) n^{\mu}+\int_{\left\{t_{-} \geq 0\right\} \cap\{t \leq s\}}\left|\partial \chi_{R}\right| \cdot\left(r^{p}|\partial \psi|^{2}+r^{p-2} \cdot|\psi|^{2}\right) .
\end{aligned}
$$

We recall that

$$
\psi_{k}(t, \cdot)=\int_{-\infty}^{+\infty} h_{k}(t-s) \cdot \psi_{t^{*}}(s, \cdot) d s,
$$

where $h_{k}$ satisfies (4.16). Hence, we can estimate for $q$ large enough (the precise value of which will be specified exactly later in the proof ):

$$
\begin{aligned}
& \int_{\left\{t=t_{1}\right\}} \chi_{R} \cdot r^{p}\left|\partial_{v} \Psi_{k}\right|^{2} d v d \sigma \\
& =\int_{\left\{t=t_{1}\right\}} r^{p} \chi_{R} \cdot\left|\int_{-\infty}^{\infty} h_{k}\left(t_{1}-s\right) \cdot \partial_{v}\left(r^{\frac{d-1}{2}} \psi_{t^{*}}\right)(s, x) d s\right|^{2} d v d \sigma \\
& \leq \int_{\left\{t=t_{1}\right\}} r^{p} \chi_{R} \cdot\left(\int_{-\infty}^{\infty} \frac{C_{q}\left(\omega_{0}\right)}{1+\left|t_{1}-s\right|^{q}}\left|\partial_{v}\left(r^{\frac{d-1}{2}} \psi_{t^{*}}\right)(s, x)\right| d s\right)^{2} d v d \sigma \\
& \leq C_{q}\left(\omega_{0}\right) \int_{\left\{t=t_{1}\right\}} r^{p} \chi_{R} \cdot\left(\int_{-\infty}^{\infty} \frac{1}{1+\left|t_{1}-s\right|^{q}}\left|\partial_{v}\left(r^{\frac{d-1}{2}} \Psi_{t^{*}}\right)(s, x)\right|^{2} d s\right) d v d \sigma \\
& \leq C_{q}\left(\omega_{0}\right) \int_{-\infty}^{\infty} \frac{1}{1+\left|t_{1}-s\right|^{q}}\left(\int_{\{t=s\}}^{\left.r^{p} \chi_{R} \cdot\left|\partial_{v}\left(r^{\frac{d-1}{2}} \psi_{t^{*}}\right)\right|^{2} d v d \sigma\right) d s}\right.
\end{aligned}
$$

and similarly

$$
\begin{aligned}
& \int_{\left\{t=t_{1}\right\}} \chi_{R} \cdot\left(r^{p}\left|r^{-1} \partial_{\sigma} \Psi_{k}\right|^{2}+\left((d-3) r^{p-2}+\min \left\{r^{p-2}, r^{-\delta}\right\}\right)\left|\Psi_{k}\right|^{2}\right) d v d \sigma \\
& \leq C_{q}\left(\omega_{0}\right) \int_{-\infty}^{\infty} \frac{1}{1+\left|t_{1}-s\right|^{q}}\left\{\int _ { \{ t = s \} } \chi _ { R } \cdot \left(r^{p}\left|r^{-1} \partial_{\sigma}\left(r^{\frac{d-1}{2}} \Psi_{t^{*}}\right)\right|^{2}\right.\right. \\
& \left.\left.\quad+\left((d-3) r^{p-2}+\min \left\{r^{p-2}, r^{-\delta}\right\}\right)\left|r^{\frac{d-1}{2}} \psi_{t^{*}}\right|^{2}\right) d v d \sigma\right\} d s
\end{aligned}
$$

In view of the fact that $\psi_{t^{*}}=h_{t^{*}} \cdot \psi$, from (5.20) and (5.21) we obtain:

$$
\begin{aligned}
& \int_{\left\{t=t_{1}\right\}} \chi_{R} \cdot\left(r^{p}\left|\partial_{v} \Psi_{k}\right|^{2}+r^{p}\left|r^{-1} \partial_{\sigma} \Psi_{k}\right|^{2}+\left((d-3) r^{p-2}\right.\right. \\
& \left.\left.\quad+\min \left\{r^{p-2}, r^{-\delta}\right\}\right)\left|\Psi_{k}\right|^{2}\right) d v d \sigma \\
& \leq C_{q}\left(\omega_{0}\right) \int_{-\infty}^{\infty} \frac{1}{1+\left|t_{1}-s\right|^{q}} \\
& \quad \times\left(\int _ { \{ t = s \} } \chi _ { R } \cdot h _ { t ^ { * } } ^ { 2 } \left(r^{p}\left|\partial_{v} \Psi\right|^{2}+r^{p}\left|r^{-1} \partial_{\sigma} \Psi\right|^{2}+\left((d-3) r^{p-2}\right.\right.\right.
\end{aligned}
$$




$$
\begin{aligned}
& \left.\left.\left.+\min \left\{r^{p-2}, r^{-\delta}\right\}\right)|\Psi|^{2}\right) d v d \sigma\right) d s \\
& +C_{q}\left(\omega_{0}\right) \int_{-\infty}^{\infty} \frac{1}{1+\left|t_{1}-s\right|^{q}}\left(\int_{\{t=s\}} \chi_{R} \cdot\left|\partial h_{t^{*}}\right|^{2} r^{p}|\Psi|^{2} d v d \sigma\right) d s
\end{aligned}
$$

Since $h_{t^{*}}$ is supported only in $\left\{t_{-} \geq 0\right\}$, due to (5.18), we can estimate for any $s \in \mathbb{R}$ :

$$
\begin{aligned}
& \int_{\{t=s\}} r^{p} \chi_{R} \cdot h_{t^{*}}^{2}\left(r^{p}\left|\partial_{v} \Psi\right|^{2}+r^{p}\left|r^{-1} \partial_{\sigma} \Psi\right|^{2}+\left((d-3) r^{p-2}\right.\right. \\
& \left.\left.\quad+\min \left\{r^{p-2}, r^{-\delta}\right\}\right)|\Psi|^{2}\right) d v d \sigma \\
& \leq C(p) \int_{t=0}\left(1+r^{p}\right) J_{\mu}^{N}(\psi) n^{\mu} \\
& \quad+C(p) \cdot \int_{\left\{\operatorname{supp}\left(\partial \chi_{R}\right)\right\} \cap\left\{0-\frac{1}{2} \chi_{1} \cdot\left(r-R_{1}\right) \leq t \leq s\right\}}\left|\partial \chi_{R}\right| \cdot\left(r^{p} J_{\mu}^{N}(\psi) n^{\mu}+r^{p-2}|\psi|^{2}\right) .
\end{aligned}
$$

In view of the inclusion $\operatorname{supp}\left(\partial \chi_{R}\right) \subseteq\{R \leq r \leq 2 R\}$, we can also bound

$$
\begin{aligned}
& \int_{\left\{\operatorname{supp}\left(\partial \mathrm{\chi}_{R}\right)\right\} \cap\left\{0-\frac{1}{2} \mathrm{\chi}_{1} \cdot\left(r-R_{1}\right) \leq t \leq s\right\}}\left|\partial \mathrm{\chi}_{R}\right| \cdot\left(r^{p} J_{\mu}^{N}(\psi) n^{\mu}+r^{p-2}|\psi|^{2}\right) \\
& \leq C\left(\mathrm{X}_{R}\right) \cdot(R+\max \{s, 0\}) \cdot \int_{t=0}\left(1+r^{p}\right) \cdot J_{\mu}^{N}(\psi) n^{\mu},
\end{aligned}
$$

and hence, from (5.23) we obtain:

$$
\begin{aligned}
& \int_{\{t=s\}} r^{p} \chi_{R} \cdot h_{t^{*}}^{2}\left(r^{p}\left|\partial_{v} \Psi\right|^{2}+r^{p}\left|r^{-1} \partial_{\sigma} \Psi\right|^{2}+\left((d-3) r^{p-2}\right.\right. \\
& \left.\left.\quad+\min \left\{r^{p-2}, r^{-\delta}\right\}\right)|\Psi|^{2}\right) d v d \sigma \\
& \quad \leq C\left(R, p, \chi_{R}\right) \cdot(1+|s|) \cdot \int_{t=0}\left(1+r^{p}\right) J_{\mu}^{N}(\psi) n^{\mu} .
\end{aligned}
$$

Since $\operatorname{supp}\left(\partial h_{t^{*}}\right) \subseteq\left\{0 \leq t_{-} \leq 1\right\} \cup\left\{t^{*}-1 \leq t_{+} \leq t^{*}\right\}$, the following inclusion

$$
\{t=s\} \cap \operatorname{supp}\left(\partial h_{t^{*}}\right) \subseteq\left\{r \leq R_{1}+C \cdot|s|\right\}
$$

holds for some $C \gg 1$. We can therefore bound through a Hardy inequality

$$
\int_{\{t=s\}} r^{p} \chi_{R} \cdot\left|\partial h_{t^{*}}\right|^{2} \cdot|\Psi|^{2} d v d \sigma \leq C \cdot \int_{\{t=s\} \cap \operatorname{supp}\left(\partial h_{t^{*}}\right) \cap\{r \geq R\}} r^{p+2} \cdot J_{\mu}^{N}(\psi) n^{\mu}
$$




$$
\begin{aligned}
& \leq C \cdot(1+|s|)^{p+2} \cdot \int_{\{t=s\} \cap \operatorname{supp}\left(\partial h_{t^{*}}\right) \cap\{r \geq R\}} J_{\mu}^{N}(\psi) n^{\mu} \\
& \leq C \cdot(1+|s|)^{p+2} \cdot \int_{\{t=0\}} J_{\mu}^{N}(\psi) n^{\mu} .
\end{aligned}
$$

Returning to (5.21) and using (5.25) and (5.26), we can finally estimate (fixing $q=6>(p+2)+2)$

$$
\begin{aligned}
& \int_{\left\{t=t_{1}\right\}} \chi_{R} \cdot\left(r^{p}\left|r^{-1} \partial_{\sigma} \Psi_{k}\right|^{2}+\left((d-3) r^{p-2}+\min \left\{r^{p-2}, r^{-\delta}\right\}\right)\left|\Psi_{k}\right|^{2}\right) d v d \sigma \\
& \leq C_{q}\left(\omega_{0}, R, p, \chi_{R}\right) \cdot\left\{\int_{-\infty}^{\infty} \frac{1+|s|^{p+2}}{1+|s|^{q}} d s\right\} \cdot \int_{t=0}\left(1+r^{p}\right) J_{\mu}^{N}(\Psi) n^{\mu} \\
& \leq C\left(\omega_{0}, R, p, \chi_{R}\right) \cdot \int_{t=0}\left(1+r^{p}\right) J_{\mu}^{N}(\psi) n^{\mu} .
\end{aligned}
$$

Thus, in view of (5.12) and Lemmas 4.3 and 4.4, from (5.27) we infer the required estimate (5.9):

$$
\begin{aligned}
& \int_{\left\{t=t_{2}\right\}} \chi_{R} \cdot\left(r^{p}\left|\partial_{v} \Psi_{k}\right|^{2}+r^{p}\left|r^{-1} \partial_{\sigma} \Psi_{k}\right|^{2}+\left((d-3) r^{p-2}\right.\right. \\
& \left.\left.\quad+\min \left\{r^{p-2}, r^{-\delta}\right\}\right)\left|\Psi_{k}\right|^{2}\right) d v d \sigma \\
& \quad+\int_{\mathcal{R}\left(t_{1}, t_{2}\right)} \chi_{R} \cdot\left(p r^{p-1}\left|\partial_{v} \Psi_{k}\right|^{2}+\left\{\left((2-p) r^{p-1}+r^{p-1-\delta}\right)\left|r^{-1} \partial_{\sigma} \Psi_{k}\right|^{2}\right.\right. \\
& \left.\left.\quad+\left((2-p)(d-3) r^{p-3}+\min \left\{r^{p-3}, r^{-1-\delta}\right\}\right)\left|\Psi_{k}\right|^{2}\right\}+r^{-1-\eta}\left|\partial_{u} \Psi_{k}\right|^{2}\right) d u d v d \sigma \\
& \leq C(p, \eta, \delta) \cdot \int_{\mathcal{R}\left(t_{1}, t_{2}\right)}\left|\partial \chi_{R}\right| \cdot\left(r^{p}\left|\partial \Psi_{k}\right|^{2}+r^{p-2}\left|\Psi_{k}\right|^{2}\right) d u d v d \sigma+ \\
& \quad+C\left(\omega_{0}, p, \eta, R, \chi_{R}, \delta\right) \cdot \int_{\{t=0\}}\left(1+r^{p}\right) \cdot J_{\mu}^{N}(\Psi) n^{\mu} .
\end{aligned}
$$

The same estimate for $\psi_{\leq \omega_{+}}, \psi_{\geq \omega_{+}}$in place of $\psi_{k}$ follows in exactly the same way. Hence, the proof of the lemma is complete

Finally, let us note that we will need to use Lemma 5.4 in order to estimate the energy of $\psi_{k}$ on the leaves of a hyperboloidal foliation restricted on $\mathcal{R}\left(0, t^{*}\right)$. Namely, we will consider the energy of $\psi_{k}$ on $\tilde{S}_{\tau} \cap \mathcal{R}\left(0, t^{*}\right)$, where the hyperboloids $\tilde{S}_{\tau}$ are defined as follows:

Definition For a fixed $\eta^{\prime}<1+2 a$, we define function $\tilde{u}_{\eta^{\prime}}:\{r \gg 1\} \rightarrow \mathbb{R}$ as

$$
\tilde{u}_{\eta^{\prime}} \doteq u-\frac{1}{1+r^{\eta^{\prime}}} .
$$


We also define for any $\tau \in \mathbb{R}$ the hypersurface $\tilde{S}_{\tau} \subset\{r \gg 1\}$ as

$$
\tilde{S}_{\tau} \doteq\left\{\tilde{u}_{\eta^{\prime}}=\tau\right\}
$$

Notice that the level sets of $\tilde{S}_{\tau}$ are indeed spacelike hypersurfaces for $r$ large enough depending on the chosen $\eta$ '. This follows from the computation:

$$
\begin{gathered}
g^{\mu \nu} \partial_{\mu} \tilde{u}_{\eta^{\prime}} \cdot \partial_{\nu} \tilde{u}_{\eta^{\prime}}=g^{\mu \nu} \partial_{\mu} u \cdot \partial_{\nu} u+2 g^{\mu \nu} \partial_{\mu} u \cdot \partial_{\nu}\left(\frac{-1}{1+r^{\eta^{\prime}}}\right) \\
+g^{\mu \nu} \partial_{\mu}\left(\frac{-1}{1+r^{\eta^{\prime}}}\right) \cdot \partial_{\nu}\left(\frac{-1}{1+r^{\eta^{\prime}}}\right) \\
=-2 \eta^{\prime} r^{-1-\eta^{\prime}}+O_{\eta^{\prime}}\left(r^{-2-a}+r^{-2-\eta^{\prime}}\right)<0 .
\end{gathered}
$$

for $r$ large enough in terms of $\eta^{\prime}$. Moreover, $\left|\tilde{u}_{\eta^{\prime}}-u\right| \leq 1$, and hence $\tilde{S}_{\tau}$ are spacelike hypersurfaces terminating at future null infinity, according to the definition (2.7).

In view of (5.29), for any smooth function $\omega:\{r \gg 1\} \rightarrow \mathbb{C}$ the following estimate is true:

$$
J_{\mu}^{N}(\omega) \tilde{n}^{\mu} \sim \frac{1}{r^{1+\eta^{\prime}}}\left|\partial_{u} \omega\right|^{2}+\left|\partial_{v} \omega\right|^{2}+\left|\frac{1}{r} \partial_{\sigma} \omega\right|^{2}
$$

where $\tilde{n}$ denotes the future directed unit normal to $\left\{\tilde{\mathrm{S}}_{\tau}\right\}_{\tau \in \mathbb{R}}$

We can now infer the following Lemma:

Lemma 5.5 Assume that $R \gg 1$ is large enough in terms of the fixed value of $\eta^{\prime}$. Then for any $\tau \in \mathbb{R}$ and any $-n \leq k \leq n$, the following inequality is true:

$$
\begin{aligned}
& \int_{0}^{\infty}\left\{\int_{\tilde{S}_{\tau} \cap \mathcal{R}\left(0, t^{*}\right) \cap\{r \geq R+1\}} J_{\mu}^{N}\left(\psi_{k}\right) \tilde{n}^{\mu}\right\} d \tau \\
& \leq C(R) \int_{\{R \leq r \leq R+1\} \cap \mathcal{R}\left(0, t^{*}\right)}\left(J_{\mu}^{N}\left(\psi_{k}\right) n^{\mu}\right. \\
& \left.\quad+\left|\psi_{k}\right|^{2}\right)+C\left(\omega_{0}, R\right) \int_{t=0}(1+r) J_{\mu}^{N}(\psi) n^{\mu} .
\end{aligned}
$$

The same estimate also holds for $\psi_{\leq \omega_{+}}, \psi_{\geq \omega_{+}}$in place of $\psi_{k}$.

Moreover, for any $t_{1} \leq t_{2}$ the following estimate for $\psi$ holds:

$$
\begin{aligned}
& \int_{t_{1}}^{t_{2}}\left\{\int_{\tilde{S}_{\tau} \cap\{r \geq R\}} J_{\mu}^{N}(\psi) \tilde{n}^{\mu}\right\} d \tau \leq C(R) \cdot \int_{\{R \leq r \leq R+1\} \cap \mathcal{R}\left(t_{1}, t_{2}\right)}\left(J_{\mu}^{N}(\psi) n^{\mu}+|\psi|^{2}\right) \\
& \quad+C \cdot \int_{\left\{t=t_{1}\right\}}(1+r) J_{\mu}^{N}(\psi) \tilde{n}^{\mu} .
\end{aligned}
$$

Proof In view of (5.31), fixing some smooth cut-off function $\chi_{R}: \mathcal{D} \rightarrow[0,1]$ such that $\chi_{R} \equiv 0$ on $\{r \leq R\}$ and $\chi_{R} \equiv 1$ on $\{r \geq R+1\}$, choosing some $0<\eta<$ $\min \left\{a, \eta^{\prime}\right\}$ and $0<\delta<1$ and setting $p=1$ in Lemma 5.4, we can readily bound for any $-n \leq k \leq n$ : 


$$
\begin{aligned}
\int_{0}^{\infty} & \left\{\int_{\tilde{\mathrm{S}}_{\tau} \cap \mathcal{R}\left(0, t^{*}\right) \cap\{r \geq R+1\}} J_{\mu}^{N}\left(\psi_{k}\right) \tilde{n}^{\mu}\right\} d \tau \\
\leq & C(R) \int_{\{R \leq r \leq R+1\} \cap \mathcal{R}\left(0, t^{*}\right)}\left(J_{\mu}^{N}\left(\psi_{k}\right) n^{\mu}+\left|\psi_{k}\right|^{2}\right) \\
& +C\left(\omega_{0}, R\right) \int_{t=0}(1+r) J_{\mu}^{N}(\psi) n^{\mu} .
\end{aligned}
$$

The same is also true for $\psi_{\leq \omega_{+}}, \psi_{\geq \omega_{+}}$in place of $\psi_{k}$.

The estimate (5.33) follows by applying Theorem 5.1 of [52] for $\psi$, adapting the proof in the region spanned by $\tilde{S}_{t_{2}}$ and $\Sigma_{t_{1}}$ (instead of the region spanned by $\tilde{S}_{t_{2}}$ and $\tilde{S}_{t_{1}}$, as is the case in the original theorem).

\section{Integrated Local Energy Decay for $\psi_{0}$}

As we explained in our sketch of the proof of Theorem 2.1 in Section 2.5, the treatment of the very low frequency component $\psi_{0}$ of $\psi$ differs substantially from the treatment of the rest of $\psi_{k}, 1 \leq|k| \leq n$. In this section, we will prove an integrated local energy decay statement for $\psi_{0}$, as described in the proposition that follows. It is in this section that the parameter $\omega_{0}$ will be fixed. Moreover, in the proof of Proposition 6.1 it is specified how small the value of $\varepsilon=\sup \{g(T, T)$ should be in the statement of Theorem 2.1.

Proposition 6.1 Suppose that $\varepsilon=\sup \{g(T, T)\}$ (defined in Assumption 3 regarding the smallness of the ergoregion; recall that, in view of our remark in Section 2.6, we have assumed that $\mathcal{H}^{+} \neq \emptyset$ ) is small enough in terms of the geometry of the region $\left\{r>\frac{r_{0}}{2}\right\}$ (i.e. the geometry of a subset of $(\mathcal{D}, g)$ not containing the ergoregion). Then for any $R>0$, there exists $a \delta=\delta(R)>0$, such that if $\omega_{0}<\delta$, we can bound:

$$
\int_{\{r \leq R\} \cap \mathcal{R}\left(0, t^{*}\right)}\left(J_{\mu}^{N}\left(\psi_{0}\right) n^{\mu}+\left|\psi_{0}\right|^{2}\right) \leq C\left(R, \omega_{0}\right) \cdot \int_{t=0} J_{\mu}^{N}(\psi) n^{\mu}
$$

Proof It suffices to establish (6.1) for $\operatorname{Re}\left(\psi_{0}\right)$ and $\operatorname{Im}\left(\psi_{0}\right)$ in place of $\psi_{0}$, since then by adding the two inequalities one arrives immediately at the statement for $\psi_{0}$. Hence, we can assume without loss of generality that $\psi_{0}$ is real valued.

Without loss of generality, we can also assume that $R$ is large enough in terms of the geometry of $(\mathcal{D}, g)$, since we can always choose an even larger $R$ than the one given in the statement.

We will need to fix a $C^{1}$ and piecewise $C^{2}$ function $h:[0,+\infty) \rightarrow[0,1]$ with the following properties for some $l>2$ :

1. For $\{r \leq 1\}: h \equiv 1$

2. For $\{1<r<2\}$ : $h^{\prime \prime}<0$ and $h^{\prime}<0$

3. For $\{2 \leq r \leq l-c\}: h(r)=\frac{3}{2 r}$ for some $0<c \ll 1$

4. For $\{l-c<r<l+c\}: h^{\prime \prime}<0, h^{\prime}<0$

5. For $\{l+c<r<2 l\}: h(r)=\frac{3}{2 l^{3}}(r-2 l)^{2}$

6. For $\{r \geq 2 l\}: h \equiv 0$ 
The existence of such a function is established as follows: First of all, the existence of a function satisfying the three first assumptions on $[0, l-c]$ and moreover satisfying $h(r)=\frac{3}{2 r}$ on the whole of $\{2 \leq r \leq l\}$ is obvious, since the only obstruction posed by the conditions $h^{\prime \prime}<0$ on $(1,2)$ and $h^{\prime}(1)=0, h^{\prime}(2)=-\frac{3}{8}$ is

$$
h^{\prime}(2) \cdot(2-1)<h(2)-h(1)
$$

And this condition is indeed verified, since $-\frac{3}{8}<-\frac{1}{4}$. Extending $h$ on the whole of $[0,2 l]$ by the expression $h(r)=\frac{3}{2 l^{3}}(r-2 l)^{2}$ on $(l, 2 l]$, we infer that

$$
\lim _{r \rightarrow l^{+}} h(r)=\frac{3}{2 l}=\lim _{r \rightarrow l^{-}} h(r)
$$

and

$$
\lim _{r \rightarrow l^{+}} h^{\prime}(r)=-\frac{3}{l^{2}}<-\frac{3}{2 l^{2}}=\lim _{r \rightarrow l^{-}} h^{\prime}(r) .
$$

Thus, we conclude that $h$ can be mollified around $r=l$ so that it is $C^{1}$ and concave around this point, and moreover $h^{\prime}<0$ in the region of the mollification. Finally, the construction of $h$ is completed by extending $h$ to be identically 0 on $[2 l,+\infty$ ) (notice that $h$ remains $C^{1}$ under this extension, since $h(2 l)=h^{\prime}(2 l)=0$ )

We can now define the function $h_{R}: \mathcal{D} \rightarrow[0,1]$ through the composition $h_{R} \doteq$ $h \circ\left(\frac{r}{R}\right)$. Using this function, we can then define the current

$$
J_{\mu}=e \cdot T_{\mu \nu}\left(\psi_{0}\right) N^{\nu}+h_{R} \psi_{0} \partial_{\mu} \psi_{0}-\frac{1}{2} \partial_{\mu} h_{R} \cdot \psi_{0}^{2}
$$

for a small parameter $e>0$ to be specified later. ${ }^{23}$ Since $\psi_{0}$ satisfies the equation $\square_{g} \psi_{0}=F_{0}$, we compute (using the abstract index notation):

$$
\nabla^{\mu} J_{\mu}=e \cdot K^{N}\left(\psi_{0}\right)+e \cdot F_{0} \cdot N \psi_{0}+h_{R} \cdot \partial^{\mu} \psi_{0} \cdot \partial_{\mu} \psi_{0}-\frac{1}{2}\left(\square_{g} h_{R}\right) \psi_{0}^{2}+h_{R} \cdot \psi_{0} \cdot F_{0}
$$

We will integrate (6.3) over $\mathcal{R}\left(0, t^{*}\right)$. Since $\psi_{0}$ is supported in some cylinder of the form $\left\{r \lesssim R_{\text {sup }}+t^{*}\right\}$ (see the remark in Section (2.6)), we need not worry about boundary terms occuring near spacelike infinty when applying the divergence theorem in $\mathcal{R}\left(0, t^{*}\right)$. After applying the divergence theorem on $\mathcal{R}\left(0, t^{*}\right)$, we obtain:

$$
\begin{gathered}
\int_{\mathcal{H}^{+} \cap \mathcal{R}\left(0, t^{*}\right)} J_{\mu} n_{\mathcal{H}^{+}}^{\mu}+\int_{\left\{t=t^{*}\right\}} J_{\mu} n^{\mu}+e \int_{\mathcal{R}\left(0, t^{*}\right)} K^{N}\left(\psi_{0}\right) \\
+\int_{\mathcal{R}\left(0, t^{*}\right)} h_{R} \partial^{\mu} \psi_{0} \cdot \partial_{\mu} \psi_{0}-\frac{1}{2} \int_{\mathcal{R}\left(0, t^{*}\right)}\left(\square_{g} h_{R}\right) \psi_{0}^{2}
\end{gathered}
$$

\footnotetext{
${ }^{23}$ Let us remark that in the case where $\mathcal{H}^{+}=\emptyset$, the proof would follow by using the current $J_{\mu}=$ $h_{R} \psi_{0} \partial_{\mu} \psi_{0}-\frac{1}{2} \partial_{\mu} h_{R} \cdot \psi_{0}^{2}$.
} 


$$
=\int_{\{t=0\}} J_{\mu} n^{\mu}-e \int_{\mathcal{R}\left(0, t^{*}\right)} F_{0} \cdot N \psi_{0}-\int_{\mathcal{R}\left(0, t^{*}\right)} h_{R} \cdot \psi_{0} \cdot F_{0},
$$

where $n_{\mathcal{H}^{+}}^{\mu}$ denotes a future directed $T$-invariant generator of $\mathcal{H}^{+} \backslash\left(\mathcal{H}^{+} \cap \mathcal{H}^{-}\right)$which coincides with the horizon Killing field $V$ on $\mathcal{H} \cap J^{+}(\Sigma)$. For the volume form involved in this integration, see Section 3 for our conventions regarding integration over null hypersurfaces.

Before proceeding to the extraction of an inequality from identity (6.4), we should note the following: Having identified $\mathcal{D} \backslash \mathcal{H}^{-}$with $\mathbb{R} \times(\Sigma \cap \mathcal{D})$ through the flow of $T$ (see Section 3.3), the inverse $g^{-1}$ of the metric $g$ (that is to say, $g^{-1}$ is the induced metric on the cotangent bundle $T^{*} \mathcal{M}$ ) splits as

$$
g^{-1}=a \cdot T \otimes T+T \otimes b+b \otimes T+\mathcal{C},
$$

where, due to our assumption 3 on the smallness of the ergoregion, the following conditions are satisfied on $\{t \geq 0\} \subset \mathcal{D}$ :

- $a$ is a real bounded function.

- $b$ is a vector field tangential to the hypersurfaces $\Sigma_{\tau}$ and uniformly bounded with respect to the induced Riemannian metric $g_{\Sigma}$ on these hypersurfaces.

- $\mathcal{C}$ is a symmetric $(0,2)$-tensor on the hypersurfaces $\Sigma_{\tau}$, uniformly bounded when measured with $g_{\Sigma}$, satisfying the lower bound $\mathcal{C} \geq-C \varepsilon \cdot g_{\Sigma}^{-1}$ in the region $\{r \leq$ $\left.\frac{3 r_{0}}{4}\right\}$ and $\mathcal{C} \geq c \cdot g_{\Sigma}^{-1}$ in the region $\left\{r \geq \frac{3 r_{0}}{4}\right\}$ for some $C, c>0$ (here we have used the notation that $A \geq B$ for two symmetric (0,2)-tensors if $A(\omega, \omega) \geq B(\omega, \omega)$ for all 1-forms $\omega$ ).

Since $K^{N}(\cdot) \gtrsim J_{\mu}^{N}(\cdot) n^{\mu}$ on $\left\{r \leq r_{0}\right\}$ (in view of Assumption 2), we can bound from below on $\Sigma_{t} \cap\left\{r \leq r_{0}\right\}$ for any $t \geq 0$ and any smooth function $v$ on $\{t \geq 0\}$ provided $e$ is chosen (independently of $t$ ) so that $e \gtrsim \varepsilon$ :

$$
e \cdot K^{N}(v)+\mathcal{C}(\partial v, \partial v) \geq 0 .
$$

Moreover, since $K^{N}(\cdot) \lesssim J_{\mu}^{N}(\cdot) n^{\mu}$ on $\left\{r_{0} \leq r \leq 2 r_{0}\right\}$ and $K^{N}$ vanishes anywhere else, we can bound on $\Sigma_{t} \cap\left\{r \geq r_{0}\right\}$ :

$$
e \cdot K^{N}(v)+\mathcal{C}(\partial v, \partial v)+(T v)^{2} \geq 0
$$

provided $e$ is chosen sufficiently small depending only on the geometric aspects of our spacetime for $\left\{r \geq r_{0}\right\}$. This assumption is compatible with the assumption $\varepsilon \lesssim e$ that we imposed for (6.6) to hold, provided that $\varepsilon$ is small enough in terms of the geometry of the spacetime region $\left\{r \geq r_{0}\right\}$.

All in all, if $\varepsilon$ is small enough in terms of the geometry of $\left\{r \geq r_{0}\right\}$, we infer that we can choose a suitable $e$ such that for any smooth function $v$ on $\{t \geq 0\}$ we can bound (from (6.6) and (6.7)):

$$
e \cdot K^{N}(v)+\mathcal{C}(\partial v, \partial v)+(T v)^{2} \geq c(e) \cdot J_{\mu}^{N}(v) n^{\mu}
$$


everywhere on $\{t \geq 0\}$. This inequality implies, due to (6.5), that for some constant $C>0$ depending on the bounds for $a, b$ in (6.5) we can estimate from below (using the abstract index notation):

$$
e \cdot K^{N}(v)+g^{\mu \nu} \partial_{\mu} v \cdot \partial_{\nu} v+C \cdot(T v)^{2} \geq c(e) \cdot J_{\mu}^{N}(v) n^{\mu}
$$

everywhere on $\{t \geq 0\}$. Therefore, from now on we can assume that that the parameter $e$ has been fixed.

Since $h_{R} \equiv 1$ near $\mathcal{H}^{+}$and $h_{R} \geq 0$ everywhere (and $K^{N}$ vanishes for $r \geq 2 r_{0}$ ), we can bound due to (6.8) (denoting with $\nabla_{\Sigma} \psi_{0}$ the gradient of $\psi_{0}$ on the $\left(\Sigma_{t}, g_{\Sigma}\right)$ hypersurfaces):

$$
e K^{N}\left(\psi_{0}\right)+h_{R} \partial^{\mu} \psi_{0} \cdot \partial_{\mu} \psi_{0} \geq c \cdot h_{R}\left|\nabla_{\Sigma} \psi_{0}\right|_{g_{\Sigma}}^{2}-C \cdot h_{R}\left|T \psi_{0}\right|^{2}
$$

Returning to the identity (6.4), in view of (6.9) we can bound:

$$
\begin{aligned}
& \int_{H^{+} \cap \mathcal{R}\left(0, t^{*}\right)} J_{\mu} n_{H^{+}}^{\mu}+\int_{\mathcal{R}\left(0, t^{*}\right)} h_{R} \cdot\left|\nabla_{\Sigma} \psi_{0}\right|_{g_{\Sigma}}^{2}-C \cdot \int_{\mathcal{R}\left(0, t^{*}\right)} h_{R} \cdot\left|T \psi_{0}\right|^{2} \\
& \quad-\frac{1}{2} \int_{\mathcal{R}\left(0, t^{*}\right)}\left(\square_{g} h_{R}\right) \psi_{0}^{2} \\
& \leq-e \int_{\mathcal{R}\left(0, t^{*}\right)} F_{0} \cdot N \psi_{0}-\int_{\mathcal{R}\left(0, t^{*}\right)} h_{R} \cdot \psi_{0} \cdot F_{0}+\int_{t=0} J_{\mu} n^{\mu}-\int_{t=t^{*}} J_{\mu} n^{\mu} .
\end{aligned}
$$

Along $\mathcal{H}^{+}$, the current $J_{\mu}$ takes the form

$$
J_{\mu}=e \cdot T_{\mu \nu}\left(\psi_{0}\right) N^{\nu}+\psi_{0} \cdot \partial_{\mu} \psi_{0}=e \cdot J_{\mu}^{N}\left(\psi_{0}\right)+\frac{1}{2} \partial_{\mu}\left(\psi_{0}^{2}\right)
$$

Therefore, we compute

$$
\begin{aligned}
-\int_{\mathcal{H}^{+} \cap \mathcal{R}\left(0, t^{*}\right)} J_{\mu} n_{\mathcal{H}^{+}}^{\mu} & =-e \int_{\mathcal{H}^{+} \cap\left\{0 \leq t \leq t^{*}\right\}} J_{\mu}^{N}\left(\psi_{0}\right) n_{\mathcal{H}^{+}}^{\mu}-\frac{1}{2} \int_{\mathcal{H}^{+} \cap\left\{0 \leq t \leq t^{*}\right\}} n_{\mathcal{H}^{+}}\left(\psi_{0}^{2}\right) \\
& \leq-\frac{1}{2} \int_{\mathcal{H}^{+} \cap\left\{0 \leq t \leq t^{*}\right\}} n_{\mathcal{H}^{+}}\left(\psi_{0}^{2}\right) \\
& =\frac{1}{2}\left(\int_{\mathcal{H}^{+} \cap\{t=0\}} \psi_{0}^{2}-\int_{\mathcal{H}^{+} \cap\left\{t=t^{*}\right\}} \psi_{0}^{2}\right)
\end{aligned}
$$

the last equality following from the definition of the volume element on $\mathcal{H}^{+}$with respect to $n_{\mathcal{H}^{+}}$, combined with the fact that $n_{\mathcal{H}^{+}}$was assumed to coincide with the horizon Killing field $V$ on $\mathcal{H}^{+} \cap J^{+}(\Sigma)$. Due to a trace theorem, a Hardy inequality and Lemma 4.4, the following estimate holds for each $\tau \in\left[0, t^{*}\right]$ : 


$$
\begin{gathered}
\int_{\{t=\tau\} \cap \mathcal{H}^{+}} \psi_{0}^{2} \leq C \cdot \int_{\{t=\tau\} \cap\{r \leq 1\}}\left(\psi_{0}^{2}+\left|\nabla_{\Sigma} \psi_{0}\right|_{g_{\Sigma}}^{2}\right) \\
\leq C \cdot \int_{t=\tau} J_{\mu}^{N}\left(\psi_{0}\right) n^{\mu} \leq C\left(\omega_{0}\right) \cdot \int_{t=0} J_{\mu}^{N}(\psi) n^{\mu} .
\end{gathered}
$$

Hence, we can bound in view of (6.12):

$$
-\int_{\mathcal{H}^{+} \cap \mathcal{R}\left(0, t^{*}\right)} J_{\mu} n_{\mathcal{H}^{+}}^{\mu} \leq C\left(\omega_{0}\right) \cdot \int_{t=0} J_{\mu}^{N}(\psi) n^{\mu}
$$

We will now estimate the boundary terms at $\left\{t=0, t^{*}\right\}$ of (6.10). Due to Lemma 4.4 (using also a Hardy inequalty for the 0-th order terms, since $h_{R}$ and $\partial h_{R}$ decay faster than $C(R) \cdot r^{-2}$, we can bound:

$$
\int_{\{t=\text { const }\}} J_{\mu}\left(\psi_{0}\right) n^{\mu} \leq C\left(\omega_{0}, R\right) \int_{\{t=0\}} J_{\mu}^{N}(\psi) n^{\mu}
$$

By substituting estimates (6.14) and (6.15) into (6.10), we infer:

$$
\begin{gathered}
\int_{\mathcal{R}\left(0, t^{*}\right)} h_{R}\left|\nabla_{\Sigma} \psi_{0}\right|_{g_{\Sigma}}^{2}-\frac{1}{2} \int_{\mathcal{R}\left(0, t^{*}\right)}\left(\square_{g} h_{R}\right) \cdot \psi_{0}^{2} \lesssim \int_{\mathcal{R}\left(0, t^{*}\right)} h_{R} \cdot\left|T \psi_{0}\right|^{2} \\
-e \int_{\mathcal{R}\left(0, t^{*}\right)} F_{0} \cdot N \psi_{0}-\int_{\mathcal{R}\left(0, t^{*}\right)} h_{R} \cdot \psi_{0} \cdot F_{0}+C\left(\omega_{0}, R\right) \int_{t=0} J_{\mu}^{N}(\psi) n^{\mu} .
\end{gathered}
$$

We will now proceed to estimate the term $\int_{\mathcal{R}\left(0, t^{*}\right)}\left(\square_{g} h_{R}\right) \cdot \psi_{0}^{2}$ in (6.16). Due to the properties of $h$, we have $\square_{g} h_{R} \equiv 0$ for $r \leq R$. We can assume without loss of generality that $R \geq R_{1}$. Then, in view of the expression (2.4) of $g$ in the $(t, r, \sigma)$ coordinate chart for $r \geq R_{1}$, we compute:

$$
\begin{aligned}
\square_{g} h_{R} & =\frac{1}{\sqrt{-\operatorname{det}(g)}} \partial_{r}\left(g^{r r} \cdot \sqrt{-\operatorname{det}(g)} \partial_{r} h_{R}\right) \\
& =\left(1+O\left(r^{-1}\right)\right)\left(\partial_{r}^{2} h_{R}+\frac{d-1}{r} \partial_{r} h_{R}\right)+O\left(r^{-1-a}\right) \cdot \partial_{r} h_{R} .
\end{aligned}
$$

Thus, we can estimate in $\{R<r<2 R\}$ (in view again of the properties of the constructed function $h$ ):

$$
\square_{g} h_{R}<-c \cdot R^{-2},
$$

provided $R \gg 1$. For $2 R<r<l R-c$ we calculate

$$
\square_{g} h_{R}=\frac{3}{2}\left\{\left(1+O\left(r^{-1}\right)\right)\left(2 R r^{-3}-\frac{d-1}{r} R r^{-2}\right)+O\left(r^{-1-a}\right) \cdot R \cdot r^{-2}\right\} \leq C \cdot \frac{R}{r^{3+a}}
$$


Similarly, for $\{l R-c<r<l R+c\}$ we can readily bound

$$
\square_{g} h_{R} \leq 0,
$$

while for $\{l R+c<r<2 l R\}$ we can estimate (provided again that $R \gg 1$ )

$$
\square_{g} h_{R} \leq \frac{C}{l^{3} R^{2}}
$$

Since $r \sim l \cdot R$ in this interval, we can estimate for any given (fixed) $0<\beta<1$ :

$$
\square_{g} h_{R} \leq C \cdot\left(\frac{(l R)^{\beta}}{l}\right) r^{-2-\beta} .
$$

It is at this point that we will determine the value of $m$ in terms of $R$ : We set $l=R^{\frac{\beta}{1-\beta}}$, and with this choice we have $\frac{(l R)^{\beta}}{l}=1$. With this choice, therefore, for $l R+c<r<2 l R$ we can estimate from (6.21):

$$
\square_{g} h_{R} \leq C \cdot r^{-2-\beta}
$$

Of course, for $r>2 l$ we have

$$
\square_{g} h_{R} \equiv 0 .
$$

Returning to (6.16) and using the above estimates for $\square h_{R}$, we can bound

$$
\begin{aligned}
& \int_{\mathcal{R}\left(0, t^{*}\right)} h_{R}\left|\nabla_{\Sigma} \psi_{0}\right|_{g_{\Sigma}}^{2}+c \cdot \int_{\mathcal{R}\left(0, t^{*}\right) \cap\{R \leq r \leq 2 R\}} \frac{\psi_{0}^{2}}{R^{2}} \leq \\
& \leq C \cdot \int_{\mathcal{R}\left(0, t^{*}\right)} h_{R} \cdot\left|T \psi_{0}\right|^{2} \\
& \quad+C \cdot \int_{\mathcal{R}\left(0, t^{*}\right) \cap\{2 R \leq r \leq l R\}} \frac{R}{r^{3+a}} \psi_{0}^{2}+C \int_{\mathcal{R}\left(0, t^{*}\right) \cap\{l R \leq r \leq 2 l R\}} \frac{\psi_{0}^{2}}{r^{2+\beta}} \\
& \quad+C\left|\int_{\mathcal{R}\left(0, t^{*}\right)} F_{0} \cdot N \psi_{0}\right|+C \cdot\left|\int_{\mathcal{R}\left(0, t^{*}\right)} h_{R} \cdot \psi_{0} \cdot F_{0}\right| \\
& \quad+C\left(\omega_{0}, R\right) \int_{t=0} J_{\mu}^{N}(\psi) n^{\mu},
\end{aligned}
$$

or, after adding a multiple of $\int_{\mathcal{R}\left(0, t^{*}\right)} h_{R} \cdot\left|T \psi_{0}\right|^{2}$ on both sides:

$$
\begin{aligned}
& \int_{\mathcal{R}\left(0, t^{*}\right)} h_{R} J_{\mu}^{N}\left(\psi_{0}\right) n^{\mu}+c \cdot \int_{\mathcal{R}\left(0, t^{*}\right) \cap\{R \leq r \leq 2 R\}} \frac{\psi_{0}^{2}}{R^{2}} \\
& \leq C \cdot \int_{\mathcal{R}\left(0, t^{*}\right)} h_{R} \cdot\left|T \psi_{0}\right|^{2}+C \cdot \int_{\mathcal{R}\left(0, t^{*}\right) \cap\{2 R \leq r \leq l R\}} \frac{R}{r^{3+a}} \psi_{0}^{2} \\
& \quad+C \int_{\mathcal{R}\left(0, t^{*}\right) \cap\{l R \leq r \leq 2 l R\}} \frac{\psi_{0}^{2}}{r^{2+\beta}}
\end{aligned}
$$




$$
\begin{aligned}
& +C\left|\int_{\mathcal{R}\left(0, t^{*}\right)} F_{0} \cdot N \psi_{0}\right|+C\left|\int_{\mathcal{R}\left(0, t^{*}\right)} h_{R} \cdot \psi_{0} \cdot F_{0}\right| \\
& +C\left(\omega_{0}, R\right) \int_{t=0} J_{\mu}^{N}(\psi) n^{\mu} .
\end{aligned}
$$

We will dispense with the third term of the right hand side of (6.25) using Lemma 5.3. According to 5.3 for $\eta=\min \left\{\frac{1}{2} \beta, a\right\}$ and $R_{c}=2 l R=2 R^{\frac{1}{1-\beta}}$, we can bound provided $R \gg 1$ :

$$
\begin{aligned}
& \int_{\mathcal{R}\left(0, t^{*}\right) \cap\{l R \leq r \leq 2 l R\}} \frac{\psi_{0}^{2}}{r^{2+\frac{1}{2} \beta}} \leq C \int_{\mathcal{R}\left(0, t^{*}\right) \cap\{R \leq r \leq 2 R\}}\left(J_{\mu}^{N}\left(\psi_{0}\right) n^{\mu}+R^{-2}\left|\psi_{0}\right|^{2}\right) \\
& +C\left(\omega_{0}, R\right) \int_{t=0} J_{\mu}^{N}(\psi) n^{\mu} .
\end{aligned}
$$

Substituting (6.26) in (6.25) we have:

$$
\begin{aligned}
& \int_{\mathcal{R}\left(0, t^{*}\right)} h_{R} J_{\mu}^{N}\left(\psi_{0}\right) n^{\mu}+c \cdot \int_{\mathcal{R}\left(0, t^{*}\right) \cap\{R \leq r \leq 2 R\}} \frac{\psi_{0}^{2}}{R^{2}} \\
& \quad \leq C \cdot \int_{\mathcal{R}\left(0, t^{*}\right)} h_{R} \cdot\left|T \psi_{0}\right|^{2}+C \cdot \int_{\mathcal{R}\left(0, t^{*}\right) \cap\{2 R \leq r \leq l R\}} \frac{R}{r^{3+a}} \psi_{0}^{2} \\
& \quad+C R^{-\frac{\beta}{2}} \int_{\{R \leq r \leq 2 R\} \cap \mathcal{R}\left(0, t^{*}\right)}\left(J_{\mu}^{N}\left(\psi_{0}\right) n^{\mu}+R^{-2} \psi_{0}^{2}\right) \\
& \quad+C\left|\int_{\mathcal{R}\left(0, t^{*}\right)} F_{0} \cdot N \psi_{0}\right|+C \cdot\left|\int_{\mathcal{R}\left(0, t^{*}\right)} h_{R} \cdot \psi_{0} \cdot F_{0}\right|+C\left(\omega_{0}, R\right) \int_{t=0} J_{\mu}^{N}(\psi) n^{\mu} .
\end{aligned}
$$

Thus, since $h_{R} \geq \frac{1}{2}$ on $\{r \leq 2 R\}$, if $R$ is large enough, the third term of the right hand side of (6.32) can be absorbed by the left hand side, yielding

$$
\begin{aligned}
& \int_{\mathcal{R}\left(0, t^{*}\right)} h_{R} J_{\mu}^{N}\left(\psi_{0}\right) n^{\mu}+c \cdot \int_{\mathcal{R}\left(0, t^{*}\right) \cap\{R \leq r \leq 2 R\}} \frac{\psi_{0}^{2}}{R^{2}} \\
& \leq C \cdot \int_{\mathcal{R}\left(0, t^{*}\right)} h_{R} \cdot\left|T \psi_{0}\right|^{2}+C \cdot \int_{\mathcal{R}\left(0, t^{*}\right) \cap\{2 R \leq r \leq l R\}} \frac{R}{r^{3+a}} \psi_{0}^{2} \\
& \quad+C\left|\int_{\mathcal{R}\left(0, t^{*}\right)} F_{0} \cdot N \psi_{0}\right|+C \cdot\left|\int_{\mathcal{R}\left(0, t^{*}\right)} h_{R} \cdot \psi_{0} \cdot F_{0}\right|+C\left(\omega_{0}, R\right) \int_{t=0} J_{\mu}^{N}(\psi) n^{\mu} .
\end{aligned}
$$

We will now absorb the second term of the righ hand side of (6.28) by the left hand side through the use of Lemma 5.2 if $R \gg 1$. According to Lemma 5.2 for any $0<\eta<\alpha$, (if $R \gg 1$ ):

$$
\begin{gathered}
\int_{\mathcal{R}\left(0, t^{*}\right) \cap\{r \geq 2 R\}} \frac{1}{r^{3+\eta}} \psi_{0}^{2} \leq C(\eta) \cdot \int_{\mathcal{R}\left(0, t^{*}\right) \cap\{R \leq r \leq 2 R\}} \\
\left\{\frac{1}{R} J_{\mu}^{N}\left(\psi_{0}\right) n^{\mu}+\frac{1}{R^{3}}\left|\psi_{0}\right|^{2}\right\}+C\left(\eta, \omega_{0}\right) \int_{t=0} J_{\mu}^{N}(\psi) n^{\mu},
\end{gathered}
$$


and hence

$$
\begin{aligned}
& \int_{\mathcal{R}\left(0, t^{*}\right) \cap\{2 R \leq r \leq l R\}} \frac{R}{r^{3+a}} \psi_{0}^{2} \leq C \cdot \int_{\mathcal{R}\left(0, t^{*}\right) \cap\{r \geq 2 R\}} \frac{R^{1-a+\eta}}{r^{3+\eta}} \psi_{0}^{2} \\
& \leq C \cdot \int_{\mathcal{R}\left(0, t^{*}\right) \cap\{R \leq r \leq 2 R\}}\left\{R^{-a+\eta} J_{\mu}^{N}\left(\psi_{0}\right) n^{\mu}+R^{-2-a+\eta}\left|\psi_{0}\right|^{2}\right\} \\
& \quad+C\left(\omega_{0}, R\right) \int_{t=0} J_{\mu}^{N}(\psi) n^{\mu} .
\end{aligned}
$$

Thus, from (6.28) and (6.30) for $\eta=\frac{a}{2}$ we deduce that:

$$
\begin{aligned}
& \int_{\mathcal{R}\left(0, t^{*}\right)} h_{R} J_{\mu}^{N}\left(\psi_{0}\right) n^{\mu}+c \cdot \int_{\mathcal{R}\left(0, t^{*}\right) \cap\{R \leq r \leq 2 R\}} R^{-2} \psi_{0}^{2} \\
& \leq C \cdot \int_{\mathcal{R}\left(0, t^{*}\right)} h_{R} \cdot\left|T \psi_{0}\right|^{2}+C \cdot \int_{\mathcal{R}\left(0, t^{*}\right) \cap\{R \leq r \leq 2 R\}}\left(R^{-\frac{a}{2}} J_{\mu}^{N}\left(\psi_{0}\right) n^{\mu}+R^{-2-\frac{a}{2}} \psi_{0}^{2}\right) \\
& \quad+C\left|\int_{\mathcal{R}\left(0, t^{*}\right)} F_{0} \cdot N \psi_{0}\right|+C \cdot\left|\int_{\mathcal{R}\left(0, t^{*}\right)} h_{R} \cdot \psi_{0} \cdot F_{0}\right|+C\left(\omega_{0}, R\right) \int_{t=0} J_{\mu}^{N}(\psi) n^{\mu},
\end{aligned}
$$

which, after absorbing the second term of the right hand side into the left hand side, yields:

$$
\begin{aligned}
& \int_{\mathcal{R}\left(0, t^{*}\right)} h_{R} \cdot J_{\mu}^{N}\left(\psi_{0}\right) n^{\mu}+c \cdot \int_{\mathcal{R}\left(0, t^{*}\right) \cap\{R \leq r \leq 2 R\}} R^{-2} \psi_{0}^{2} \leq C \cdot \int_{\mathcal{R}\left(0, t^{*}\right)} h_{R} \cdot\left|T \psi_{0}\right|^{2} \\
& +C\left|\int_{\mathcal{R}\left(0, t^{*}\right)} F_{0} \cdot N \psi_{0}\right|+C \cdot\left|\int_{\mathcal{R}\left(0, t^{*}\right)} h_{R} \cdot \psi_{0} \cdot F_{0}\right|+C\left(\omega_{0}, R\right) \int_{t=0} J_{\mu}^{N}(\psi) n^{\mu}
\end{aligned}
$$

Fixing a smooth $\chi:[0,+\infty) \rightarrow[0,1]$ such that $\chi \equiv 1$ on $r \leq 1$ and $\chi \equiv 0$ on $r \geq 2$, by applying a Poincare inequality for the compactly supported function $\left(\chi \circ\left(\frac{r}{R}\right)\right) \cdot \psi_{0}$ we obtain

$$
\begin{aligned}
\int_{\mathcal{R}\left(0, t^{*}\right) \cap\{r \leq R\}} \psi_{0}^{2} & \leq C \cdot R^{2} \int_{\mathcal{R}\left(0, t^{*}\right) \cap\{r \leq 2 R\}}\left|\nabla_{\Sigma}\left\{\psi_{0} \cdot\left(\chi \circ\left(\frac{r}{R}\right)\right)\right\}\right|_{g_{\Sigma}}^{2} \leq \quad \text { (6.33) } \\
& \leq C(R)\left(\int_{\mathcal{R}\left(0, t^{*}\right) \cap\{r \leq 2 R\}}\left|\nabla_{\Sigma} \psi_{0}\right|_{g_{\Sigma}}^{2}+\int_{\mathcal{R}\left(0, t^{*}\right) \cap\{R \leq r \leq 2 R\}}\left|\psi_{0}\right|^{2}\right) .
\end{aligned}
$$

Therefore, (6.32) can be upgraded to:

$$
\begin{aligned}
& \int_{\mathcal{R}\left(0, t^{*}\right)} h_{R} \cdot J_{\mu}^{N}\left(\psi_{0}\right) n^{\mu}+\int_{\mathcal{R}\left(0, t^{*}\right) \cap\{r \leq 2 R\}} \psi_{0}^{2} \leq C(R) \cdot \int_{\mathcal{R}\left(0, t^{*}\right)} h_{R} \cdot\left|T \psi_{0}\right|^{2} \\
& \quad+C(R)\left|\int_{\mathcal{R}\left(0, t^{*}\right)} F_{0} \cdot N \psi_{0}\right|+C(R) \cdot\left|\int_{\mathcal{R}\left(0, t^{*}\right)} h_{R} \cdot \psi_{0} \cdot F_{0}\right| \\
& \quad+C\left(\omega_{0}, R\right) \int_{t=0} J_{\mu}^{N}(\psi) n^{\mu} .
\end{aligned}
$$


We will now absorb the first term of the right hand side of (6.34) by the left hand side under the condition that $\omega_{0}$ is small in terms of $R$. Recall that, due to Lemma 4.8, we can bound ${ }^{24}$ :

$$
\int_{\mathcal{R}\left(0, t^{*}\right)} h_{R} \cdot\left|T \psi_{0}\right|^{2} \leq C \cdot \omega_{0}^{2} \int_{\mathcal{R}\left(0, t^{*}\right) \cap\{r \leq 2 l R\}} \psi_{0}^{2}+C\left(\omega_{0}, R\right) \int_{t=0} J_{\mu}^{N}(\psi) n^{\mu} .
$$

Since, due to Lemma 5.2, we can bound

$$
\begin{aligned}
& \int_{\mathcal{R}\left(0, t^{*}\right) \cap\{2 R \leq r \leq 2 l R\}} \psi_{0}^{2} \leq C(R) \cdot \int_{\mathcal{R}\left(0, t^{*}\right) \cap\{R \leq r \leq 2 R\}}\left(J_{\mu}^{N}\left(\psi_{0}\right) n^{\mu}+\psi_{0}^{2}\right) \\
& +C\left(\omega_{0}, R\right) \int_{t=0} J_{\mu}^{N}(\psi) n^{\mu},
\end{aligned}
$$

the inequality (6.35) yields

$$
\begin{aligned}
& \int_{\mathcal{R}\left(0, t^{*}\right)} h_{R} \cdot\left|\partial_{t} \psi_{0}\right|^{2} \leq C(R) \cdot \omega_{0}^{2} \int_{\mathcal{R}\left(0, t^{*}\right) \cap\{r \leq 2 R\}}\left(J_{\mu}^{N}\left(\psi_{0}\right) n^{\mu}+\psi_{0}^{2}\right) \\
& \quad+C\left(\omega_{0}, R\right) \int_{t=0} J_{\mu}^{N}(\psi) n^{\mu} .
\end{aligned}
$$

Substituting (6.38) in (6.34), we obtain:

$$
\begin{aligned}
& \int_{\mathcal{R}\left(0, t^{*}\right)} h_{R} \cdot J_{\mu}^{N}\left(\psi_{0}\right) n^{\mu}+\int_{\mathcal{R}\left(0, t^{*}\right) \cap\{r \leq 2 R\}} \psi_{0}^{2} \\
& \leq C(R) \cdot \omega_{0}^{2} \int_{\mathcal{R}\left(0, t^{*}\right) \cap\{r \leq 2 R\}}\left(J_{\mu}^{N}\left(\psi_{0}\right) n^{\mu}+\psi_{0}^{2}\right) \\
& \quad+C(R)\left|\int_{\mathcal{R}\left(0, t^{*}\right)} F_{0} \cdot N \psi_{0}\right|+C(R) \cdot\left|\int_{\mathcal{R}\left(0, t^{*}\right)} h_{R} \cdot \psi_{0} \cdot F_{0}\right| \\
& \quad+C\left(\omega_{0}, R\right) \int_{t=0} J_{\mu}^{N}(\psi) n^{\mu} .
\end{aligned}
$$

Hence, if $\delta$ in the statement of the current Proposition (and thus $\omega_{0}$ ) is small enough in terms of the given $R$, the first term of the right hand side of (6.38) can be absorbed into the left hand side ( since $h_{R} \geq c>0$ on $\{r \leq 2 R\}$ ). Thus, we deduce:

$$
\begin{aligned}
& \int_{\mathcal{R}\left(0, t^{*}\right)} h_{R} \cdot J_{\mu}^{N}\left(\psi_{0}\right) n^{\mu}+\int_{\mathcal{R}\left(0, t^{*}\right) \cap\{r \leq 2 R\}} \psi_{0}^{2} \\
& \leq C(R)\left|\int_{\mathcal{R}\left(0, t^{*}\right)} F_{0} \cdot N \psi_{0}\right|+C(R) \cdot\left|\int_{\mathcal{R}\left(0, t^{*}\right)} h_{R} \cdot \psi_{0} \cdot F_{0}\right| \\
& \quad+C\left(\omega_{0}, R\right) \int_{t=0} J_{\mu}^{N}(\psi) n^{\mu} .
\end{aligned}
$$

24 Note that $\operatorname{supp}\left(h_{R}\right) \subseteq\{r \leq 2 l R\}=\left\{r \leq 2 R^{\frac{1}{1-\beta}}\right\}$. 
Finally, as we noted before, we can bound due to Lemma 5.2:

$$
\begin{aligned}
& \int_{\mathcal{R}\left(0, t^{*}\right) \cap\{r \geq 2 R\}} r^{-2}\left|N \psi_{0}\right|^{2}+r^{-4} \psi_{0}^{2} \\
& C \cdot \int_{\mathcal{R}\left(0, t^{*}\right) \cap\{R \leq r \leq 2 R\}}\left(J_{\mu}^{N}\left(\psi_{0}\right) n^{\mu}+\psi_{0}^{2}\right)+C\left(\omega_{0}, R\right) \int_{t=0} J_{\mu}^{N}(\psi) n^{\mu}
\end{aligned}
$$

and thus we can estimate

$$
\begin{aligned}
& \int_{\mathcal{R}\left(0, t^{*}\right)}(1+r)^{-2}\left|N \psi_{0}\right|^{2}+(1+r)^{-4} \psi_{0}^{2} \\
& \quad \leq C \cdot \int_{\mathcal{R}\left(0, t^{*}\right) \cap\{r \leq 2 R\}}\left(J_{\mu}^{N}\left(\psi_{0}\right) n^{\mu}+\psi_{0}^{2}\right)+C\left(\omega_{0}, R\right) \int_{t=0} J_{\mu}^{N}(\psi) n^{\mu} .
\end{aligned}
$$

Therefore, by a Cauchy-Schwarz inequality and an application of Lemma 4.3, we can bound

$$
\begin{aligned}
& C(R)\left|\int_{\mathcal{R}\left(0, t^{*}\right)} F_{0} \cdot N \psi_{0}\right|+C(R) \cdot\left|\int_{\mathcal{R}\left(0, t^{*}\right)} h_{R} \cdot \psi_{0} \cdot F_{0}\right| \\
& \leq\left\{C \cdot \int_{\mathcal{R}\left(0, t^{*}\right) \cap\{r \leq 2 R\}}\left(J_{\mu}^{N}\left(\psi_{0}\right) n^{\mu}+\psi_{0}^{2}\right)\right. \\
& \left.\quad+C\left(\omega_{0}, R\right) \int_{t=0} J_{\mu}^{N}(\psi) n^{\mu}\right\}^{1 / 2} \cdot\left\{C\left(\omega_{0}, R\right) \int_{t=0} J_{\mu}^{N}(\psi) n^{\mu}\right\}^{1 / 2} .
\end{aligned}
$$

Substituting in (6.39) and absorbing the $J_{\mu}^{N}\left(\psi_{0}\right) n^{\mu}+\psi_{0}^{2}$ term of the first factor in (6.42) into the left hand side of (6.39), we conclude:

$$
\begin{aligned}
& \int_{\mathcal{R}\left(0, t^{*}\right)} h_{R} J_{\mu}^{N}\left(\psi_{0}\right) n^{\mu}+\int_{\mathcal{R}\left(0, t^{*}\right) \cap\{r \leq 2 R\}} \psi_{0}^{2} \\
& \quad \leq C\left(\omega_{0}, R\right) \int_{t=0} J_{\mu}^{N}(\psi) n^{\mu} .
\end{aligned}
$$

Thus, the proof of the Proposition is complete.

\section{Integrated Local Energy Decay for $\psi_{\leq \omega_{+}}$}

In this section, we will establish an integrated local energy decay estimate for the low frequency part $\psi_{\leq \omega_{+}}$of $\psi$. This will be accomplished by using of a suitable Carleman type inequality and an ODE lemma appearing in [58]. Of course we only have to obtain an integrated local energy decay for $\psi_{k}$ with $1 \leq|k| \leq n$, since the related estimate for the very low frequency part $\psi_{0}$ was obtained in Section 6. 


\subsection{A Carleman-Type Estimate for $\psi_{k}, 1 \leq|k| \leq n$}

We will need to establish a Carleman type inequality for the functions $\psi_{k}, 1 \leq|k| \leq n$, that will be necessary for proving the full integrated local energy decay statement for $\psi_{\leq \omega_{+}}$in the next section. The main ideas contained in the proof of the following lemma have already been presented and used in [58].

Lemma 7.1 There exist functions $w, w^{\prime}: \mathcal{D} \rightarrow[0,1]$, satisfying $T(w)=T\left(w^{\prime}\right)=0$ and which for $r \geq R_{1}$ are both equal and strictly increasing functions of the variable $r$, such that for any $R>0$, any $0<\omega_{0} \ll 1$, any $\omega_{+}>1$ and any $1 \leq|k| \leq n$ the following bound holds (setting $s=C\left(\omega_{0}, R\right) \cdot \omega_{k}$ for some large constant $C\left(\omega_{0}, R\right)$ depending only on $\omega_{0}$ and $R$ ):

$$
\begin{aligned}
& \int_{\mathcal{R}\left(0, t^{*}\right) \cap\{r \leq R\}}\left(e^{2 s w}+e^{2 s w^{\prime}}\right) \cdot\left(J_{\mu}^{N}\left(\psi_{k}\right) n^{\mu}+\left|\psi_{k}\right|^{2}\right) \\
& \leq C\left(\omega_{0}, R\right) \cdot \int_{\left.\mathcal{R}\left(0, t^{*}\right) \cap\{R \leq r \leq R+1\}\right)}\left(1+s^{2}\right) e^{2 s w} \cdot\left(J_{\mu}^{N}\left(\psi_{k}\right) n^{\mu}+\left|\psi_{k}\right|^{2}\right) \\
& \quad+e^{C(R) \cdot s} \cdot C\left(\omega_{0}, R\right) \int_{t=0} J_{\mu}^{N}(\psi) n^{\mu} .
\end{aligned}
$$

Proof We will assume without loss of generality that $R$ is large enough in terms of the geometry of $(\mathcal{D}, g)$, since we can always pick a larger $R$ if necessary. Recall that we have assumed without loss of generality that $\mathcal{H}^{+} \neq \varnothing$ (see Section 2.6). The proof of (7.1) in the case where $\mathcal{H}^{+}=\varnothing$ is almost identical, and we will explain in the footnotes the differences of the proof in the two cases.

For any smooth function $w: \mathcal{D} \rightarrow \mathbb{R}$ and some real number $s \in \mathbb{R}$ to be fixed more precisely later on (it will eventually agree with the parameter $s$ in the statement of the lemma), we define, in accordance with [58], the following current for any $1 \leq|k| \leq n$ using the abstract index notation:

$$
\begin{aligned}
J_{\mu}= & \partial^{\nu}\left(e^{2 s w}\right) T_{\mu \nu}\left(\psi_{k}\right)+\frac{1}{2} \square_{g} e^{2 s w} \cdot \operatorname{Re}\left\{\bar{\psi}_{k} \cdot \partial_{\mu} \psi_{k}\right\} \\
& +\frac{1}{2} s\left\{\left(\partial_{\mu} w\right)\left(\square_{g} e^{2 s w}\right)-\left(\partial_{\mu} \square_{g} w\right) e^{2 s w}\right\}\left|\psi_{k}\right|^{2} .
\end{aligned}
$$

The divergence of (7.2) takes the form:

$$
\begin{aligned}
\nabla^{\mu} J_{\mu}= & \left\{s^{2}\left(\square_{g} w\right)^{2}+4 s^{3} \partial^{\mu} w \cdot \partial_{\mu} w \cdot\left(\square_{g} w\right)+4 s^{2} \nabla_{\mu \nu}^{2} w \cdot \partial^{\mu} w \cdot \partial^{\nu} w\right. \\
& \left.+4 s^{4}\left(\partial^{\mu} w \cdot \partial_{\mu} w\right)^{2}-\frac{1}{2} s \square_{g}^{2} w\right\} e^{2 s w}\left|\Psi_{k}\right|^{2} \\
& +\left\{4 s^{2} \partial_{\mu} w \cdot \square_{g} w+4 s^{2} \nabla_{\mu \nu}^{2} w \cdot \partial^{\nu} w+8 s^{3} \partial^{\nu} w \cdot \partial_{\nu} w \cdot \partial_{\mu} w\right\} e^{2 s w} \operatorname{Re}\left\{\bar{\psi}_{k} \cdot \partial^{\mu} \Psi_{k}\right\} \\
& +\left\{2 s \nabla_{\mu \nu}^{2} w+4 s^{2} \partial_{\mu} w \cdot \partial_{\nu} w\right\} e^{2 s w} \partial^{\mu} \bar{\psi}_{k} \partial^{\nu} \psi_{k} \\
& +\frac{1}{2}\left(\square_{g} e^{2 s w}\right) \operatorname{Re}\left\{\bar{\psi}_{k} \cdot F_{k}\right\}+\partial^{\mu} e^{2 s w} \cdot \operatorname{Re}\left\{\partial_{\mu} \bar{\psi}_{k} \cdot F_{k}\right\} .
\end{aligned}
$$


Proceeding as in [58], after completion of the square, (7.3) becomes:

$$
\begin{aligned}
\nabla^{\mu} J_{\mu}= & e^{2 s w}\left|2 s \partial^{\mu} w \cdot \partial_{\mu} \psi_{k}+\left(2 s^{2} \partial^{\mu} w \cdot \partial_{\mu} w+s \cdot \square_{g} w\right) \cdot \psi_{k}\right|^{2} \\
& +2 s \nabla_{\mu \nu}^{2} w \cdot \partial^{\mu}\left(e^{s w} \bar{\psi}_{k}\right) \cdot \partial^{\nu}\left(e^{s w} \psi_{k}\right) \\
& +2 s^{3} \nabla_{\mu \nu}^{2} w \cdot \partial^{\mu} w \cdot \partial^{\nu} w \cdot e^{2 s w}\left|\psi_{k}\right|^{2}-\frac{1}{2} s\left(\square_{g}^{2} w\right) \cdot e^{2 s w}\left|\psi_{k}\right|^{2} \\
& +\frac{1}{2}\left(\square_{g} e^{2 s w}\right) \operatorname{Re}\left\{\bar{\psi}_{k} \cdot F_{k}\right\}+\partial^{\mu} e^{2 s w} \cdot \operatorname{Re}\left\{\partial_{\mu} \bar{\psi}_{k} \cdot F_{k}\right\} .
\end{aligned}
$$

Let $\chi(x): \Sigma \rightarrow[0,1]$ be a smooth cut-off function to be defined more precisely later on, with compact support away from $\mathcal{H}^{+} \cap \Sigma$. We extend it to the whole of $\mathcal{D} \backslash \mathcal{H}^{-}$ by the condition $T \chi=0$. Then the divergence identity yields:

$$
\int_{\mathcal{R}\left(0, t^{*}\right)} \chi \cdot \nabla^{\mu} J_{\mu}=\int_{\left\{t=t^{*}\right\}} \chi \cdot J_{\mu} n^{\mu}-\int_{\{t=0\}} \chi \cdot J_{\mu} n^{\mu}-\int_{\mathcal{R}\left(0, t^{*}\right)} \partial^{\mu} \chi \cdot J_{\mu} .
$$

Substituting (7.4) in (7.5) and setting (for convenience)

$$
U_{k} \doteq e^{s w}\left(2 s \partial^{\mu} w \cdot \partial_{\mu} \psi_{k}+\left(2 s^{2} \partial^{\mu} w \cdot \partial_{\mu} w+s \cdot \square_{g} w\right) \cdot \psi_{k}\right)
$$

we obtain the following Carleman identity (in accordance with the Riemannian setting in [58]):

$$
\begin{aligned}
& \int_{\mathcal{R}\left(0, t^{*}\right)} \chi \cdot\left|U_{k}\right|^{2}+2 s \int_{\mathcal{R}\left(0, t^{*}\right)} \chi \nabla_{\mu \nu}^{2} w \cdot \partial^{\mu}\left(e^{s w} \bar{\psi}_{k}\right) \cdot \partial^{\nu}\left(e^{s w} \psi_{k}\right) \\
& \quad+2 s^{3} \int_{\mathcal{R}\left(0, t^{*}\right)} \chi \nabla_{\mu \nu}^{2} w \cdot \partial^{\mu} w \cdot \partial^{\nu} w \cdot e^{2 s w}\left|\Psi_{k}\right|^{2} \\
& =\frac{1}{2} s \int_{\mathcal{R}\left(0, t^{*}\right)} \chi\left(\square_{g}^{2} w\right) \cdot e^{2 s w}\left|\psi_{k}\right|^{2}+\int_{\mathcal{R}\left(0, t^{*}\right)} \chi R e\left\{e^{s w} U_{k} \cdot \bar{F}_{k}\right\} \\
& \quad+\int_{\left\{t=t^{*}\right\}} \chi \cdot J_{\mu} n^{\mu}-\int_{\{t=0\}} \chi \cdot J_{\mu} n^{\mu}-\int_{\mathcal{R}\left(0, t^{*}\right)} \partial^{\mu} \chi \cdot J_{\mu} .
\end{aligned}
$$

Using a Cauchy-Schwarz inequality

$$
\int_{\mathcal{R}\left(0, t^{*}\right)} \chi \operatorname{Re}\left\{e^{s w} U_{k} \cdot \bar{F}_{k}\right\} \leq\left(\int_{\mathcal{R}\left(0, t^{*}\right)} \chi e^{2 s w}\left|F_{k}\right|^{2}\right)^{1 / 2}\left(\int_{\mathcal{R}\left(0, t^{*}\right)} \chi\left|U_{k}\right|^{2}\right)^{1 / 2},
$$

after absorbing the term $\int_{\mathcal{R}\left(0, t^{*}\right)} \chi\left|U_{k}\right|^{2}$ into the corresponding term on the left hand side of (7.7) and then dropping once and for all the positive $\int_{\mathcal{R}\left(0, t^{*}\right)} \chi\left|U_{k}\right|^{2}$ term from the left hand side, we obtain the following inequality:

$$
2 s \int_{\mathcal{R}\left(0, t^{*}\right)} \chi \operatorname{Hess}_{\mu \nu}(w) \cdot \partial^{\mu}\left(e^{s w} \bar{\psi}_{k}\right) \cdot \partial^{\nu}\left(e^{s w} \psi_{k}\right)
$$




$$
\begin{aligned}
& +2 s^{3} \int_{\mathcal{R}\left(0, t^{*}\right)} \chi \nabla_{\mu \nu}^{2} w \cdot \partial^{\mu} w \cdot \partial^{\nu} w \cdot e^{2 s w}\left|\Psi_{k}\right|^{2} \leq \\
\leq & \frac{1}{2} s \int_{\mathcal{R}\left(0, t^{*}\right)} \chi\left(\square_{g}^{2} w\right) \cdot e^{2 s w}\left|\Psi_{k}\right|^{2}+C \cdot \int_{\mathcal{R}\left(0, t^{*}\right)} \chi e^{2 s w}\left|F_{k}\right|^{2} \\
& +\int_{\left\{t=t^{*}\right\}} \chi \cdot J_{\mu} n^{\mu}-\int_{\{t=0\}} \chi \cdot J_{\mu} n^{\mu}-\int_{\mathcal{R}\left(0, t^{*}\right)} \partial^{\mu} \chi \cdot J_{\mu} .
\end{aligned}
$$

We will now proceed to define more precisely the function $w$. In order to be able to control the boundary terms in the above inequality, we will impose the condition $T w=0$, and thus $w$ will be uniquely determined by its restriction on $\Sigma \cap \mathcal{D}=\{t=0\}$.

If it were possible to construct a geodesically convex function $w$ (that is, one with positive definite Hessian), inequality (7.8) would readily yield the desired local integrated decay statement for $\psi_{k}$. However, in general the construction of such a function $w$ is not possible for the class of manifolds under consideration, as, for example, even the existence of a single geodesic of $\mathcal{D}$ contained in a subset of the form $\{r \leq R\}$ would exclude the existence of such a $w$. See also the relevant comments in [58]. For this reason, we will construct the function $w$ so that it is geodesically convex along the gradient direction (thus guaranteeing the positivity of the second term of the left hand side of (7.8)), at least away from the ergoregion, and away from a discrete set of points which will be the necessary local extrema of $w$, which are necessitated by the lack of serious restrictions on the topology of $\Sigma$. This approach was originally adopted in the Carleman-type estimates established in [58].

According to [58] (see also [8]), we can construct a Morse function $\omega: \Sigma \rightarrow \mathbb{R}$, such that $\varphi$ has no local minima in $\Sigma$, and such that moreover $\omega \equiv r$ for $(\{r \leq$ $\left.\left.2 r_{0}\right\} \cup\left\{r \geq R_{1}\right\}\right) \cap \Sigma$. For instance, $\omega$ can be obtained by first solving the Dirichlet problem

$$
\left\{\begin{array}{l}
\Delta \bar{\omega}=-1 \text { on }\left\{2 r_{0}<r<R_{1}\right\} \cap \Sigma \\
\bar{\omega}=2 r_{0} \text { on } r=2 r_{0} \\
\bar{\omega}=R_{1} \text { on } r=R_{1},
\end{array}\right.
$$

where $\Delta$ is the Laplacian of the induced Riemannian metric on $\Sigma$. Such a function $\bar{\omega}$ is smooth on $\left\{2 r_{0} \leq r \leq R_{1}\right\} \cap \Sigma$, and has no local minima in $\left\{2 r_{0}<r<R_{1}\right\} \cap \Sigma$, since if such a local minimum existed then we should have $\Delta \bar{\omega} \geq 0$ there. The set of Morse functions is dense in the $C^{2}$ topology, and thus we can approximate $\bar{\omega}$ in $C^{2}$ by a Morse function $\omega$ on $\left\{2 r_{0} \leq r \leq R_{1}\right\} \cap \Sigma$. In view of the maximum principle and Hopf's lemma, we must have

$$
2 r_{0}<\bar{\omega}<R_{1} \text { on }\left\{2 r_{0}<r<R_{1}\right\} \cap \Sigma
$$

and

$$
\left.\partial_{r} \bar{\omega}\right|_{\left\{r=2 r_{0}\right\} \cap \Sigma},\left.\partial_{r} \bar{\omega}\right|_{\left\{r=R_{1}\right\} \cap \Sigma}>0 .
$$

Note that $d r \neq 0$ in $\left\{r \leq 3 r_{0}\right\}$, and hence the expression $\left.\partial_{r} \bar{\omega}\right|_{\left\{r=2 r_{0}\right\} \cap \Sigma}>0$ makes sense in any local chart around the horizon where $r$ is a coordinate function; similarly 
for the expression $\left.\partial_{r} \bar{\omega}\right|_{\left\{r=R_{1}\right\} \cap \Sigma}>0$. Hence, if $\omega$ was chosen close enough to $\bar{\omega}$, $\omega$ can be mollified around $\left(\left\{r=2 r_{0}\right\} \cup\left\{r=R_{1}\right\}\right) \cap \Sigma$, so that it can be smoothly extended as a Morse function equal to $r$ on $\left(\left\{r \leq 2 r_{0}\right\} \cup\left\{r \geq R_{1}\right\}\right) \cap \Sigma$. In this way, we obtain a Morse function $\omega$ with no local minima in $\Sigma$ and such that $\omega \equiv r$ for $\left(\left\{r \leq 2 r_{0}\right\} \cup\left\{r \geq R_{1}\right\}\right) \cap \Sigma .^{25}$

Since $\omega$ is a Morse function, it only has a finite number of critical points $\left\{x_{j}\right\}_{j=1}^{n}$, all lying in the region $\left\{2 r_{0} \leq r \leq R_{1}\right\} \cap \Sigma$, and there exist positive numbers $\left\{\bar{\varepsilon}_{j}\right\}_{j=1}^{n}$ such that the balls $\left\{B\left(x_{j}, \bar{\varepsilon}_{j}\right)\right\}_{j=1}^{n}$ are disjoint (the balls considered with respect to the induced Riemannian metric $g_{\Sigma}$ on $\Sigma$ ).

We will also need to construct a second Morse function $\omega^{\prime}$ with the same properties as $\omega$, but with a different set of points of local extrema. Due to the construction of $\omega$ none of the $x_{j}$ 's are points of local minima, and thus there exist points $y_{j} \in B\left(x_{j}, \frac{1}{2} \bar{\varepsilon}_{j}\right)$ such that $\omega\left(y_{j}\right)>\omega\left(x_{j}\right)$. We can find a diffeomorphism $\zeta: \Sigma \rightarrow \Sigma$ which is equal to the identity outside $\cup_{j=1}^{n} B\left(x_{j}, \frac{1}{2} \bar{\varepsilon}_{j}\right)$, and which interchanges $x_{j}$ with $y_{j}$. Considering, hence, the function

$$
\omega^{\prime}=\omega \circ \zeta
$$

we infer that $\omega \equiv \omega^{\prime}$ on $\Sigma \backslash \cup_{j=1}^{n} B\left(x_{j}, \frac{1}{2} \bar{\varepsilon}_{j}\right)$, and the only critical points of $\omega^{\prime}$ are $y_{j}$. Moreover there exist positive numbers $\left\{\varepsilon_{j}\right\}_{j=1}^{n}$ such that $\omega<\omega^{\prime}$ on $\cup_{j=1}^{n} B\left(x_{j}, \varepsilon_{j}\right)$ and $\omega^{\prime}<\omega$ on $\cup_{j=1}^{n} B\left(y_{j}, \varepsilon_{j}\right)$, with $\cup_{j=1}^{n} B\left(x_{j}, \varepsilon_{j}\right)$ and $\cup_{j=1}^{n} B\left(y_{j}, \varepsilon_{j}\right)$ being disjoint.

Extending $\omega$ to the whole of $\mathcal{D} \backslash \mathcal{H}^{-}$by the condition $T \omega=0$, and setting $w=e^{\lambda \omega}$, for some $\lambda>0$ to be determined later, we compute

$$
\nabla_{\mu \nu}^{2} w \cdot \partial^{\mu} w \partial^{\nu} w=\left\{\lambda^{2}\left(\partial^{\mu} \omega \cdot \partial_{\mu} \omega\right)^{2}+\lambda \operatorname{Hess}_{\mu \nu}(\omega) \partial^{\mu} \omega \cdot \partial^{\nu} \omega\right\} e^{3 \lambda \omega} .
$$

Moreover, for any vector field $X \in \Gamma(T \Sigma)$, we calculate

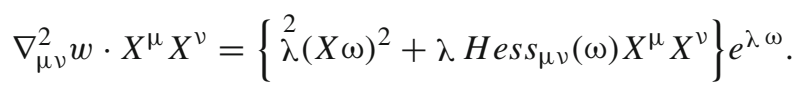

Notice that $\partial^{\mu} \omega \cdot \partial_{\mu} \omega \neq 0$ on the spacetime region $\left\{r>\frac{1}{2} r_{0}\right\} \backslash\left(\mathbb{R} \times \cup_{j=1}^{n}\left\{x_{j}\right\}\right){ }^{26}$ This is due to the fact that outside the ergoregion, the level sets of $\omega$ will always contain the timelike tangent vector $T$, and hence their normal will never be a null vector. Thus, $\partial^{\mu} \omega \cdot \partial_{\mu} \omega \neq 0$ whenever $\partial_{\mu} \omega$ is non zero in $\left\{r>\frac{1}{2} r_{0}\right\}$. Hence, for any $R \gg 1$, there exists a large enough $\lambda=\lambda(R)$ such that in the region $\left\{\frac{3 r_{0}}{4} \leq r \leq\right.$ $R+1\} \backslash\left(\mathbb{R} \times \cup_{j=1}^{n} B\left(x_{j}, \frac{1}{4} \varepsilon_{j}\right)\right):$

$$
\nabla_{\mu \nu}^{2} w \cdot \partial^{\mu} w \partial^{\nu} w \geq 1>0 .
$$

After fixing an auxiliary Riemannian metric $h$ on $\mathcal{D}$ which is $T$ invariant and equal to the Euclidean metric inherited by the chart we have fixed near the asymptotically

\footnotetext{
25 In the case where $\mathcal{H}^{+}=\emptyset$, a similar construction yields a Morse function $\omega$ such that $\omega \equiv r$ on $\left\{r \geq R_{1}\right\} \cap \Sigma$.

26 Recall that we have identified $\mathcal{D} \backslash \mathcal{H}^{-}$with $\mathbb{R} \times(\Sigma \cap \mathcal{D})$.
} 
flat end (i.e. the chart $(t, r, \sigma)$ chart where $g$ takes the form (2.4)), we can also bound in the region $\left\{\frac{3 r_{0}}{4} \leq r \leq R+1\right\} \backslash\left(\mathbb{R} \times \cup_{j=1}^{n} B\left(x_{j}, \frac{1}{4} \varepsilon_{j}\right)\right)$ due to (7.12) for any fixed small $c_{0}>0$ (having chosen $\lambda=\lambda\left(R, c_{0}\right)$ large enough):

$$
\nabla_{\mu \nu}^{2} w \cdot X^{\mu} X^{\nu} \geq-c_{0} \cdot\left(\nabla_{\mu \nu}^{2} w \frac{\partial^{\mu} w \cdot \partial^{\nu} w}{|\partial w|_{h}^{2}}\right)|X|_{h}^{2} .
$$

That is, the negative part of the Hessian of $w$ can be controlled by the value of the Hessian in the gradient direction. ${ }^{27}$

We will denote for simplicity the Hessian in the gradient direction as

$$
\nabla_{n n}^{2} w \doteq \nabla_{\mu \nu}^{2} w \frac{\partial^{\mu} w \cdot \partial^{\nu} w}{|\partial w|_{h}^{2}}
$$

Then, (7.14) and a Cauchy-Schwarz inequality imply that:

$$
\nabla_{\mu \nu}^{2} w \cdot \partial^{\mu}\left(e^{s w} \bar{\psi}_{k}\right) \cdot \partial^{\nu}\left(e^{s w} \psi_{k}\right) \geq-2 c_{0} \nabla_{\mu \nu}^{2} w e^{2 s w}\left(s^{2}|\partial w|_{h}^{2}\left|\psi_{k}\right|^{2}+\left|\partial \psi_{k}\right|_{h}^{2}\right) .
$$

We will now fix the cut-off function $\chi$ appearing in (7.8). Fixing a smooth function $\chi=\chi_{R}: \Sigma \rightarrow[0,1]$ such that $\chi_{R} \equiv 0$ on $\left\{r \leq \frac{3 r_{0}}{4}\right\} \cup\{r \geq R+1\} \cup_{j=1}^{n}$ $B\left(x_{j}, \frac{1}{4} \varepsilon_{j}\right)$ and $\chi_{R} \equiv 1$ on $\left\{\frac{7 r_{0}}{8} \leq r \leq R\right\} \backslash \cup_{j=1}^{n} B\left(x_{j}, \frac{1}{2} \varepsilon_{j}\right)$, extending it on $\mathcal{D} \backslash \mathcal{H}^{-}$ by the condition $T \chi_{R}=0$, we deduce from (7.8), in view also of (7.13), (7.14) and (7.16):

$$
\begin{aligned}
(1- & \left.2 c_{0}\right) \cdot s^{3} \int_{\mathcal{R}\left(0, t^{*}\right)} \chi_{R} \nabla_{n n}^{2} w \cdot|\partial w|_{h}^{2} e^{2 s w}\left|\Psi_{k}\right|^{2} \\
\leq & C \cdot s \int_{\mathcal{R}\left(0, t^{*}\right)} \chi_{R}\left(\square_{g}^{2} w\right) \cdot e^{2 s w}\left|\psi_{k}\right|^{2} \\
& +2 c_{0} s \cdot \int_{\mathcal{R}\left(0, t^{*}\right)} \chi_{R} \nabla_{n n}^{2} w \cdot e^{2 s w}\left|\partial \psi_{k}\right|_{h}^{2}+ \\
& +\int_{\mathcal{R}\left(0, t^{*}\right)} \chi_{R} e^{2 s w}\left|F_{k}\right|^{2}+\int_{\left\{t=t^{*}\right\}} \chi_{R} \cdot J_{\mu} n^{\mu} \\
& -\int_{\{t=0\}} \chi_{R} \cdot J_{\mu} n^{\mu}-\int_{\mathcal{R}\left(0, t^{*}\right)} \partial^{\mu} \chi_{R} \cdot J_{\mu} .
\end{aligned}
$$

In view of Lemmas 4.3 and 4.4 (using also some Hardy-type inequality to control the 0 -th order terms on the slices $\left.t=0, t^{*}\right)$, we obtain from 7.17 after dividing with $\left(1-2 c_{0}\right)$ (provided $c_{0}$ was chosen so that $c_{0} \leq \frac{1}{4}$ ):

\footnotetext{
${ }^{27}$ In the case where $\mathcal{H}^{+}=\emptyset$, in view of the fact that in this case $g(T, T)<0$ everywhere on $\mathcal{D}$ according to Assumption 3, we can similarly arrange so that (7.13) and (7.14) hold on the whole of $\{r \leq R+1\} \backslash\left(\mathbb{R} \times \cup_{j=1}^{n} B\left(x_{j}, \frac{1}{4} \varepsilon_{j}\right)\right)$.
} 


$$
\begin{aligned}
& s^{3} \int_{\mathcal{R}\left(0, t^{*}\right)} \chi_{R} \nabla_{n n}^{2} w \cdot|\partial w|_{h}^{2} e^{2 s w}\left|\psi_{k}\right|^{2} \\
& \leq C \cdot s \int_{\mathcal{R}\left(0, t^{*}\right)} \chi_{R}\left(\square_{g}^{2} w\right) \cdot e^{2 s w}\left|\psi_{k}\right|^{2} \\
& \left.\quad+\frac{2 c_{0}}{1-2 c_{0}} \cdot s \cdot \int_{\mathcal{R}\left(0, t^{*}\right)} \chi_{R} \nabla_{n n}^{2} w \cdot e^{2 s w}\left|\partial \psi_{k}\right|_{h}^{2}\right\} \\
& \quad+C(R) \cdot \int_{\mathcal{R}\left(0, t^{*}\right) \cap\{\operatorname{supp}(\partial \chi)\}}\left(1+s^{2}\right) e^{2 s w}\left\{\left|\partial \psi_{k}\right|_{h}^{2}+\left|\psi_{k}\right|^{2}\right\} \\
& \quad+e^{C(R) \cdot s} \cdot C\left(\omega_{0}, R\right) \cdot \int_{t=0} J_{\mu}^{N}(\psi) n^{\mu} .
\end{aligned}
$$

We will now proceed to absorb the first two terms of the righ hand side of (7.18) into the left hand side after choosing $s$ sufficirently large. We will use the auxiliary current

$$
J_{\mu}^{a u x}=\nabla_{n n}^{2} w \cdot e^{2 s w} \cdot \operatorname{Re}\left\{\bar{\psi}_{k} \cdot \partial_{\mu} \psi_{k}\right\}-\frac{1}{2} \partial_{\mu}\left\{\nabla_{n n}^{2} w \cdot e^{2 s w}\right\}\left|\psi_{k}\right|^{2},
$$

for which the divergence theorem yields

$$
\begin{aligned}
& \int_{\mathcal{R}\left(0, t^{*}\right)} \chi_{R} \cdot\left(\nabla_{n n}^{2} w \cdot e^{2 s w} \cdot \partial^{\mu} \bar{\psi}_{k} \cdot \partial_{\mu} \psi_{k}-\frac{1}{2} \square_{g}\left\{\nabla_{n n}^{2} w \cdot e^{2 s w}\right\} \cdot\left|\psi_{k}\right|^{2}\right) \\
& =-\int_{\mathcal{R}\left(0, t^{*}\right)} \nabla_{n n}^{2} w \cdot e^{2 s w} \cdot \operatorname{Re}\left\{\bar{\psi}_{k} \cdot F_{k}\right\}-\int_{t=t^{*}} \chi_{R} \cdot J_{\mu}^{\text {aux }} n^{\mu} \\
& \quad+\int_{t=0} \chi_{R} \cdot J_{\mu}^{\text {aux }} n^{\mu}-\int_{\mathcal{R}\left(0, t^{*}\right)} \partial^{\mu} \chi_{R} \cdot J_{\mu}^{\text {aux }} .
\end{aligned}
$$

Therefore, from (7.20) we obtain the following Langrangean identity:

$$
\begin{aligned}
& \int_{\mathcal{R}\left(0, t^{*}\right)} \chi_{R} \cdot \nabla_{n n}^{2} w \cdot e^{2 s w} \cdot \partial^{\mu} \bar{\psi}_{k} \cdot \partial_{\mu} \psi_{k} \\
& =\frac{1}{2} \int_{\mathcal{R}\left(0, t^{*}\right)} \chi_{R} \cdot \square_{g}\left\{\nabla_{n n}^{2} w \cdot e^{2 s w}\right\} \cdot\left|\psi_{k}\right|^{2} \\
& \quad-\int_{\mathcal{R}\left(0, t^{*}\right)} \nabla_{n n}^{2} w \cdot e^{2 s w} \cdot \operatorname{Re}\left\{\bar{\psi}_{k} \cdot F_{k}\right\}-\int_{t=t^{*}} \chi_{R} \cdot J_{\mu}^{\text {aux }} n^{\mu} \\
& \quad+\int_{t=0} \chi_{R} \cdot J_{\mu}^{\text {aux }} n^{\mu}-\int_{\mathcal{R}\left(0, t^{*}\right)} \partial^{\mu} \chi_{R} \cdot J_{\mu}^{\text {aux }} .
\end{aligned}
$$

In order to estimate the second term of the right hand side of (7.18), we will use the trivial bound

$$
\left|\partial \psi_{k}\right|_{h}^{2} \leq C_{1} \cdot \partial^{\mu} \bar{\psi}_{k} \cdot \partial_{\mu} \psi_{k}+C_{2}\left|T \psi_{k}\right|^{2} .
$$

After using Lemmas 4.3 and 4.4 to deal with the $F_{k}$-terms and the boundary terms in (7.21) (as we did in the derivation of (7.18)), from (7.18), (7.21) and Lemma 4.8 (which allows us to bound $\left|T \psi_{k}\right|^{2}$ bulk terms by $\omega_{k}^{2}\left|\psi_{k}\right|^{2}$ ) terms we obtain: 


$$
\begin{aligned}
& s^{3} \int_{\mathcal{R}\left(0, t^{*}\right)} \chi_{R} \cdot \nabla_{n n}^{2} w \cdot|\partial w|_{h}^{2} e^{2 s w}\left|\Psi_{k}\right|^{2} \leq C \cdot s \int_{\mathcal{R}\left(0, t^{*}\right)} \chi_{R} \cdot\left(\square_{g}^{2} w\right) \cdot e^{2 s w}\left|\Psi_{k}\right|^{2} \\
& +C\left(\omega_{0}\right) \cdot s \cdot \omega_{k}^{2} \int_{\mathcal{R}\left(0, t^{*}\right)} \chi_{R} \nabla_{n n}^{2} w \cdot e^{2 s w}\left|\psi_{k}\right|^{2} \\
& +C \cdot \frac{2 c_{0}}{1-2 c_{0}} \cdot s \cdot \int_{\mathcal{R}\left(0, t^{*}\right)} \chi_{R} \cdot \square_{g}\left\{\nabla_{n n}^{2} w \cdot e^{2 s w}\right\} \cdot\left|\psi_{k}\right|^{2} \\
& +C\left(\omega_{0}, R\right) \int_{\mathcal{R}\left(0, t^{*}\right) \cap\left\{\operatorname{supp}\left(\partial \chi_{R}\right)\right\}}\left(1+s^{2}\right) e^{2 s w}\left\{\left|\partial \psi_{k}\right|_{h}^{2}+\left|\psi_{k}\right|^{2}\right\} \\
& +e^{C(R) \cdot s} \cdot C\left(\omega_{0}, R\right) \int_{t=0} J_{\mu}^{N}(\psi) n^{\mu} .
\end{aligned}
$$

In view of the fact that in the support of $\chi_{R}$ we can bound $\nabla_{n n}^{2} w \cdot|\partial w|_{h}^{2} \geq 1$ (due to (7.13)), the third term of the right hand side of (7.23) can be bounded as follows:

$$
\begin{aligned}
& C \cdot \frac{2 c_{0}}{1-2 c_{0}} \cdot s \cdot \int_{\mathcal{R}\left(0, t^{*}\right)} \chi_{R} \cdot \square_{g}\left\{\nabla_{n n}^{2} w \cdot e^{2 s w}\right\} \cdot\left|\psi_{k}\right|^{2} \\
& \quad \leq C(R) \cdot \frac{2 c_{0}}{1-2 c_{0}} \cdot\left(s+s^{3}\right) \cdot \int_{\mathcal{R}\left(0, t^{*}\right)} \chi_{R} \cdot \nabla_{n n}^{2} w \cdot|\partial w|_{h}^{2} e^{2 s w}\left|\psi_{k}\right|^{2} .
\end{aligned}
$$

Thus, if $c_{0}$ is small enough in terms of $R$ (which due to (7.14) corresponds to $\lambda$ having been chosen large enough in terms of $R$ ), the third term of the right hand of (7.23) can be absorbed into the left hand side. Moreover, if we set $s=C \cdot \omega_{k}$, where $C=C\left(R, \omega_{0}\right)>0$ is large enough in terms of $R, \omega_{0}$, the left hand side of (7.23) can also absorb the first two terms of the right hand side, yielding the following Carleman type inequality:

$$
\begin{aligned}
& s^{3} \int_{\mathcal{R}\left(0, t^{*}\right)} \chi_{R} e^{2 s w}\left|\psi_{k}\right|^{2} \leq C\left(\omega_{0}, R\right) \cdot \int_{\mathcal{R}\left(0, t^{*}\right) \cap\{\operatorname{supp}(\partial \chi)\}}\left(1+s^{2}\right) e^{2 s w}\left\{\left|\partial \psi_{k}\right|_{h}^{2}+\left|\psi_{k}\right|^{2}\right\} \\
& +e^{C(R) \cdot s} \cdot C\left(\omega_{0}, R\right) \int_{t=0} J_{\mu}^{N}(\psi) n^{\mu} .
\end{aligned}
$$

In view of the Lagrangean identity (7.21), we can also upgrade inequality 7.25 to:

$$
\begin{aligned}
& s^{3} \int_{\mathcal{R}\left(0, t^{*}\right)} \chi_{R} e^{2 s w}\left|\psi_{k}\right|^{2}+c\left(\omega_{0}, R\right) \cdot s \int_{\mathcal{R}\left(0, t^{*}\right)} \chi_{R} e^{2 s w}\left|\partial \psi_{k}\right|_{h}^{2} \\
& \leq C\left(\omega_{0}, R\right) \int_{\mathcal{R}\left(0, t^{*}\right) \cap\left\{\operatorname{supp}\left(\partial \chi_{R}\right)\right\}}\left(1+s^{2}\right) e^{2 s w}\left\{\left|\partial \psi_{k}\right|_{h}^{2}+\left|\psi_{k}\right|^{2}\right\} \\
& \quad+e^{C(R) \cdot s} \cdot C\left(\omega_{0}, R\right) \int_{t=0} J_{\mu}^{N}(\psi) n^{\mu}
\end{aligned}
$$

for some constant $c\left(\omega_{0}, R\right)$ small in terms of $\omega_{0}, R$.

The support of $\partial \chi_{R}$ in $\mathcal{D} \backslash \mathcal{H}^{-}$breaks into three pieces: The part contained in $\mathbb{R} \times\left(\cup_{j=1}^{n} B\left(x_{j}, \varepsilon_{j}\right)\right)$, the part contained in $\left\{\frac{3 r_{0}}{4} \leq r \leq \frac{7 r_{0}}{8}\right\}$ and the part contained in 
$\{R \leq r \leq R+1\}$. Thus, the first term of the right hand side of (7.26) naturally splits into three summands (corresponding to the aforementioned partition of $\operatorname{supp}\left(\partial \chi_{R}\right)$ ):

$$
\begin{aligned}
& \int_{\mathcal{R}\left(0, t^{*}\right) \cap\left\{\operatorname{supp}\left(\partial \mathrm{\chi}_{R}\right)\right\}}\left(1+s^{2}\right) e^{2 s w}\left\{\left|\partial \psi_{k}\right|_{h}^{2}+\left|\psi_{k}\right|^{2}\right\} \\
& =\int_{\mathcal{R}\left(0, t^{*}\right) \cap\left\{\operatorname{supp}\left(\partial \mathrm{\chi}_{R}\right)\right\} \cap\left(\mathbb{R} \times\left(\cup_{j=1}^{n} B\left(x_{j}, \varepsilon_{j}\right)\right)\right)}\left(1+s^{2}\right) e^{2 s w}\left\{\left|\partial \psi_{k}\right|_{h}^{2}+\left|\psi_{k}\right|^{2}\right\} \\
& \quad+\int_{\mathcal{R}\left(0, t^{*}\right) \cap\left\{\operatorname{supp}\left(\partial \chi_{R}\right)\right\} \cap\left\{\frac{3 r_{0}}{4} \leq r \leq \frac{7 r_{0}}{8}\right\}}\left(1+s^{2}\right) e^{2 s w}\left\{\left|\partial \psi_{k}\right|_{h}^{2}+\left|\psi_{k}\right|^{2}\right\} \\
& \quad+\int_{\mathcal{R}\left(0, t^{*}\right) \cap\left\{\operatorname{supp}\left(\partial \chi_{R}\right)\right\} \cap\{R \leq r \leq R+1\}}\left(1+s^{2}\right) e^{2 s w}\left\{\left|\partial \psi_{k}\right|_{h}^{2}+\left|\psi_{k}\right|^{2}\right\} .
\end{aligned}
$$

Therefore, in order to reach a statement close to (7.1), we have to dispense with the first two summands of the right hand side of (7.27). ${ }^{28}$ We will accomplish this task in two steps.

1. In order to deal with the first term of the right hand side of (7.27), we will make use of the second Morse function function $\omega^{\prime}$ defined as (7.10).

By defining $w^{\prime}=e^{\lambda \omega^{\prime}}$ and $\chi_{R}^{\prime}=\chi_{R} \circ \zeta$, and repeating the same procedure as before (leading to (7.26)) with $w^{\prime}$ and $\chi_{R}^{\prime}$ in place of $w$ and $\chi_{R}$, we obtain the inequality

$$
\begin{aligned}
& s^{3} \int_{\mathcal{R}\left(0, t^{*}\right)} \chi_{R}^{\prime} e^{2 s w^{\prime}}\left|\psi_{k}\right|^{2}+c\left(\omega_{0}, R\right) \int_{\mathcal{R}\left(0, t^{*}\right)} \chi_{R}^{\prime} e^{2 s w^{\prime}}\left|\partial \psi_{k}\right|_{h}^{2} \\
& \leq C\left(\omega_{0}, R\right) \int_{\mathcal{R}\left(0, t^{*}\right) \cap\left\{\operatorname{supp}\left(\partial \chi_{R}^{\prime}\right)\right\}}\left(1+s^{2}\right) e^{2 s w^{\prime}}\left\{\left|\partial \psi_{k}\right|_{h}^{2}+\left|\psi_{k}\right|^{2}\right\} \\
& \quad+e^{C(R) \cdot s} \cdot C\left(\omega_{0}, R\right) \int_{t=0} J_{\mu}^{N}(\psi) n^{\mu} .
\end{aligned}
$$

Recall that $\varphi<\varphi^{\prime}$ on $\cup_{j=1}^{n} B\left(x_{j}, \varepsilon_{j}\right)$ and $\omega^{\prime}<\omega$ on $\cup_{j=1}^{n} B\left(y_{j}, \varepsilon_{j}\right)$, with $\cup_{j=1}^{n} B\left(x_{j}, \varepsilon_{j}\right)$ and $\cup_{j=1}^{n} B\left(y_{j}, \varepsilon_{j}\right)$ being disjoint. This condition guarantees that if the constant $C\left(\omega_{0}, R\right)$ in the definition $s=C\left(\omega_{0}, R\right) \cdot \omega_{k}$ is chosen large enough, then the term

$$
\int_{\mathcal{R}\left(0, t^{*}\right) \cap\left\{\operatorname{supp}\left(\partial \chi_{R}\right)\right\} \cap\left(\mathbb{R} \times\left(\cup_{j=1}^{n} B\left(x_{j}, \varepsilon_{j}\right)\right)\right)}\left(1+s^{2}\right) e^{2 s w}\left\{\left|\partial \psi_{k}\right|_{h}^{2}+\left|\psi_{k}\right|^{2}\right\}
$$

of (7.26) can be controlled by the left hand side of (7.28), while the term

$$
\int_{\mathcal{R}\left(0, t^{*}\right) \cap\left\{\operatorname{supp}\left(\partial \mathrm{x}_{R}^{\prime}\right)\right\} \cap\left(\mathbb{R} \times\left(\cup_{j=1}^{n} B\left(y_{j}, \varepsilon_{j}\right)\right)\right)}\left(1+s^{2}\right) e^{2 s w^{\prime}}\left\{\left|\partial \psi_{k}\right|_{h}^{2}+\left|\psi_{k}\right|^{2}\right\}
$$

\footnotetext{
28 Notice that the same steps apply in the case where $\mathcal{H}^{+}=\emptyset$, the only difference being that in that case the second term of the right hand side of (7.27) can be dropped.
} 
of (7.28) can be controlled by the left hand side of 7.26. Therefore, recalling that $w=w^{\prime}$ on the complement of $\mathbb{R} \times \cup_{j=1}^{n} B\left(x_{j}, \frac{1}{2} \bar{\varepsilon}_{j}\right)$, and redefining $\chi_{R}$ to be equal to the maximum of the previous two cut-offs $\chi_{R}, \chi_{R}^{\prime}{ }^{29}$, we obtain after adding (7.26) and (7.28):

$$
\begin{aligned}
& \int_{\mathcal{R}\left(0, t^{*}\right)} \chi_{R} \cdot\left(e^{2 s w}+e^{2 s w^{\prime}}\right)\left|\psi_{k}\right|^{2}+\int_{\mathcal{R}\left(0, t^{*}\right)} \chi_{R} \cdot\left(e^{2 s w}+e^{2 s w^{\prime}}\right)\left|\partial \psi_{k}\right|_{h}^{2} \\
& \leq C\left(\omega_{0}, R\right) \int_{\mathcal{R}\left(0, t^{*}\right) \cap\left(\left\{\frac{3 r_{0}}{4} \leq r \leq \frac{7 r_{0}}{8}\right\} \cup\{R \leq r \leq R+1\}\right)}\left(1+s^{2}\right) e^{2 s w}\left\{\left|\partial \psi_{k}\right|_{h}^{2}+\left|\psi_{k}\right|^{2}\right\} \\
& \quad+e^{C(R) \cdot s} \cdot C\left(\omega_{0}, R\right) \int_{t=0} J_{\mu}^{N}(\psi) n^{\mu} .
\end{aligned}
$$

2. In order to deal with the first term of the right hand side of (7.29), we will use the red shift current $K^{N}$, which is positive definite near the horizon (see Assumption 2). This will also help us extend our control of the inegral of $\left|\psi_{k}\right|^{2}+\left|\partial \psi_{k}\right|_{h}^{2}$ on the whole of $\{r \leq R\}$. $^{30}$

Setting $\tilde{\chi}_{R}=\max \left(1_{\left\{0 \leq r \leq r_{0}\right\}}, \chi_{R}\right)$ (note that $\tilde{\chi}_{R} \equiv 1$ on $\{r \leq R\}$ ), we compute using the divergence theorem:

$$
\begin{aligned}
& \int_{\mathcal{R}\left(0, t^{*}\right)} \tilde{\chi}_{R} K^{N}\left(\psi_{k}\right)=-\int_{t=t^{*}} \tilde{\chi}_{R} J_{\mu}^{N}\left(\psi_{k}\right) n^{\mu} \\
& -\int_{\mathcal{H}^{+} \cap \mathcal{R}\left(0, t^{*}\right)} \tilde{\chi}_{R} J_{\mu}^{N}\left(\psi_{k}\right) n^{\mu}+\int_{t=0} \tilde{\chi}_{R} J_{\mu}^{N}\left(\psi_{k}\right) n^{\mu} \\
& -\int_{\mathcal{R}\left(0, t^{*}\right)} \partial^{\mu} \tilde{\chi}_{R} \cdot J_{\mu}^{N}\left(\psi_{k}\right)-\int_{\mathcal{R}\left(0, t^{*}\right)} \tilde{\chi}_{R} \operatorname{Re}\left\{N \bar{\psi}_{k} \cdot F_{k}\right\} .
\end{aligned}
$$

Hence, in view of (2.8), Lemmas 4.3 and 4.4, and a Cauchy-Schwarz inequality for the term $\int_{\mathcal{R}\left(0, t^{*}\right)} \tilde{\chi}_{R} \operatorname{Re}\left\{N \bar{\psi}_{k} \cdot F_{k}\right\}$, we obtain from (7.30) for any small $\delta>0$ :

$$
\begin{gathered}
\int_{R\left(0, t^{*}\right) \cap\left\{r \leq \frac{7}{8} r_{0}\right\}}\left|\partial \psi_{k}\right|_{h}^{2} \leq C \cdot \int_{\mathcal{R}\left(0, t^{*}\right) \cap\left\{r_{0} \leq r \leq 2 r_{0}\right\}}\left|\partial \psi_{k}\right|_{h}^{2}+\delta \cdot \int_{\mathcal{R}\left(0, t^{*}\right)} \chi_{R}\left|\partial \psi_{k}\right|_{h}^{2} \\
+C\left(\omega_{0}\right) \delta^{-1} \int_{t=0} J_{\mu}^{N}\left(\psi_{k}\right) n^{\mu}+C(R) \cdot \int_{\mathcal{R}\left(0, t^{*}\right) \cap\{R \leq r \leq R+1\}}\left\{\left|\partial \psi_{k}\right|_{h}^{2}+\left|\psi_{k}\right|^{2}\right\} .
\end{gathered}
$$

Hence, after adding (7.31) multiplied by $\sup _{\left\{r \leq 7 r_{0} / 8\right\}} e^{2 s w}$ to (7.29) for some $\delta$ small enough in terms of $\omega_{0}, R$, we infer:

\footnotetext{
${ }^{29}$ Note that in this way, we have $\chi_{R} \equiv 1$ on the whole of $\left\{\frac{7 r_{0}}{8} \leq r \leq R\right\}$.

${ }^{30}$ Notice that this step is unnecessary in the case where $\mathcal{H}^{+}=\emptyset$, as in that case the second term of the right hand side of (7.27) can be dropped.
} 


$$
\begin{aligned}
& \int_{\mathcal{R}\left(0, t^{*}\right) \cap\left\{\frac{7 r_{0}}{8} \leq r \leq R\right\}}\left(e^{2 s w}+e^{2 s w^{\prime}}\right)\left|\psi_{k}\right|^{2}+\int_{\mathcal{R}\left(0, t^{*}\right) \cap\{r \leq R\}}\left(e^{2 s w}+e^{2 s w^{\prime}}\right)\left|\partial \psi_{k}\right|_{h}^{2} \\
& \leq C\left(\omega_{0}, R\right) \cdot\left(\sup _{\left\{r \leq 7 r_{0} / 8\right\}} e^{2 s w}\right) \int_{\mathcal{R}\left(0, t^{*}\right) \cap\left\{r_{0} \leq r \leq 2 r_{0}\right\}}\left|\partial \psi_{k}\right|_{h}^{2} \\
& +C\left(\omega_{0}, R\right) \int_{\left.\mathcal{R}\left(0, t^{*}\right) \cap\{R \leq r \leq R+1\}\right)}\left(1+s^{2}\right) e^{2 s w}\left\{\left|\partial \psi_{k}\right|_{h}^{2}+\left|\psi_{k}\right|^{2}\right\} \\
& +e^{C(R) \cdot s} \cdot C\left(\omega_{0}, R\right) \int_{t=0} J_{\mu}^{N}(\psi) n^{\mu} \text {. }
\end{aligned}
$$

Since, due to the construction of $\omega$, we have

$$
\sup _{\left\{r \leq 7 r_{0} / 8\right\}} w<\inf _{\left\{r_{0} \leq r \leq 2 r_{0}\right\}} w
$$

for $s=C\left(\omega_{0}, R\right) \cdot \omega_{k}$ large enough the first term of the right hand side of (7.32) can be absorbed into the second term of the left hand side, yielding:

$$
\begin{aligned}
& \int_{\mathcal{R}\left(0, t^{*}\right) \cap\left\{\frac{7 r_{0}}{8} \leq r \leq R\right\}}\left(e^{2 s w}+e^{2 s w^{\prime}}\right) \cdot\left|\psi_{k}\right|^{2}+\int_{\mathcal{R}\left(0, t^{*}\right) \cap\{r \leq R\}}\left(e^{2 s w}+e^{2 s w^{\prime}}\right) \cdot\left|\partial \psi_{k}\right|_{h}^{2} \\
& \leq C\left(\omega_{0}, R\right) \int_{\left.\mathcal{R}\left(0, t^{*}\right) \cap\{R \leq r \leq R+1\}\right)}\left(1+s^{2}\right) e^{2 s w}\left\{\left|\partial \psi_{k}\right|_{h}^{2}+\left|\psi_{k}\right|^{2}\right\} \\
& \quad+e^{C(R) \cdot s} \cdot C\left(\omega_{0}, R\right) \int_{t=0} J_{\mu}^{N}(\psi) n^{\mu}
\end{aligned}
$$

Estimating through a Hardy-type inequality

$$
\begin{aligned}
\int_{\mathcal{R}\left(0, t^{*}\right) \cap\left\{r \leq \frac{7}{8} r_{0}\right\}}\left|\psi_{k}\right|^{2}= & \int_{\mathcal{R}\left(0, t^{*}\right) \cap\left\{r \leq \frac{7}{8} r_{0}\right\}}\left|\tilde{\mathrm{\chi}}_{R} \psi_{k}\right|^{2} \\
\leq & C(R) \int_{\mathcal{R}\left(0, t^{*}\right)} \tilde{\mathrm{\chi}}_{R}\left|\partial \psi_{k}\right|_{h}^{2}+C(R) \int_{\mathcal{R}\left(0, t^{*}\right)}\left|\partial \tilde{\chi}_{R}\right|_{h}^{2}\left|\psi_{k}\right|^{2} \\
\leq & C(R) \int_{\mathcal{R}\left(0, t^{*}\right) \cap\{r \leq R\}}\left|\partial \psi_{k}\right|_{h}^{2} \\
& +C(R) \int_{\mathcal{R}\left(0, t^{*}\right) \cap\{R \leq r \leq R+1\}}\left\{\left|\partial \psi_{k}\right|_{h}^{2}+\left|\psi_{k}\right|^{2}\right\}
\end{aligned}
$$

after adding (7.35) and (7.34) we obtain the desired inequality:

$$
\begin{aligned}
& \int_{\mathcal{R}\left(0, t^{*}\right) \cap\{r \leq R\}}\left(e^{2 s w}+e^{2 s w^{\prime}}\right) \cdot\left(\left|\psi_{k}\right|^{2}+\left|\partial \psi_{k}\right|_{h}^{2}\right) \\
& \quad \leq C\left(\omega_{0}, R\right) \int_{\left.\mathcal{R}\left(0, t^{*}\right) \cap\{R \leq r \leq R+1\}\right)}\left(1+s^{2}\right) e^{2 s w}\left\{\left|\partial \psi_{k}\right|_{h}^{2}+\left|\psi_{k}\right|^{2}\right\} \\
& \quad+e^{C(R) \cdot s} \cdot C\left(\omega_{0}, R\right) \int_{t=0} J_{\mu}^{N}(\psi) n^{\mu} .
\end{aligned}
$$




\subsection{Integrated Local Energy Decay for $\psi_{\leq \omega_{+}}$}

In this section, we will remove the first term of the right hand side of (7.1) from Lemma 7.1, in order to obtain a genuine integrated local energy decay statement for $\psi_{k}, 1 \leq|k| \leq n$.

The main argument involved in the proof of Proposition 7.2 originates in [58]. In particular, we will use an ODE Lemma stated and proven in Sections 9 and 10 of [58]. We will mainly work in the region $\{r \gg 1\}$, where in the $(t, r, \sigma)$ coordinate chart, according to Assumption 1 (see also Section 2.6 on our simplifying assumption that this region has a single connected component), the metric takes the form

$$
\begin{aligned}
g= & -\left(1-\frac{2 M}{r}+O_{4}\left(r^{-1-a}\right)\right) d t^{2}+\left(1+\frac{2 M}{r}+O_{4}\left(r^{-1-a}\right)\right) d r^{2} \\
& +r^{2}\left(g_{\mathbb{S}^{d-1}}+O_{4}\left(r^{-1-a}\right)\right)+O_{4}\left(r^{-a}\right) d t d \sigma .
\end{aligned}
$$

We will first establish the following Proposition:

Proposition 7.2 For any $R>0$ and $0<\omega_{0} \ll 1$, there exists a positive constant $C=C\left(R, \omega_{0}\right)$ such that for any smooth solution $\psi$ to the wave equation on $J^{+}(\Sigma) \cap D$ with compactly supported initial data on $\{t=0\}$, any $\omega_{+}>1$ and any $1 \leq|k| \leq n$ we can bound:

$$
\int_{\{r \leq R\} \cap \mathcal{R}\left(0, t^{*}\right)}\left\{J_{\mu}^{N}\left(\psi_{k}\right) n^{\mu}+\left|\psi_{k}\right|^{2}\right\} \leq C\left(R, \omega_{0}\right) \cdot e^{C\left(R, \omega_{0}\right) \cdot \omega_{+}} \int_{t=0} J_{\mu}^{N}(\psi) n^{\mu} .
$$

Proof We will assume without loss of generality that $1 \leq k \leq n$, since the case $-n \leq k \leq-1$ follows in exactly the same way. We will also assume without loss of generality that $\psi_{k}$ is real valued (since otherwise we can first establish (7.38) first for $\operatorname{Re}\left(\psi_{k}\right)$ and $\operatorname{Im}\left(\psi_{k}\right)$ and then add the resulting inequalities).

The proof of the estimate (7.38) will follow from a number of auxilliary lemmas that aim to provide us with control over the boundary term near $r \sim R$ in the right hand side of (7.1). To this end, we introduce a large constant $C_{1}=C_{1}\left(R, \omega_{0}\right)$, the magnitude of which will be defined more precisely later in the proof. We will examine the behavior of $\int_{\{r=\rho\} \cap \mathcal{R}\left(0, t^{*}\right)}\left|\psi_{k}\right|^{2}$ in the region $\rho \geq C_{1}$.

We will make the following normalization, so that our notations are in agreement with [58]: We will set (provided, of course, $\psi_{k}$ is not identically 0 on $\mathcal{R}\left(0, t^{*}\right)$, in which case (7.38) would follow immediately)

$$
\tilde{\psi}_{k}=\left(\int_{\mathcal{R}\left(0, t^{*}\right)}(1+r)^{-2}\left|\psi_{k}\right|^{2}\right)^{-1 / 2} \psi_{k}
$$

and

$$
\tilde{F}_{k}=\left(\int_{\mathcal{R}\left(0, t^{*}\right)}(1+r)^{-2}\left|\psi_{k}\right|^{2}\right)^{-1 / 2} F_{k}
$$

Notice that $\tilde{\psi}_{k}$ solves $\square_{g} \tilde{\psi}_{k}=\tilde{F}_{k}$. 
We will also set, in order to stay close to the notations of [58]:

$$
\mathfrak{D} \doteq\left(\int_{\mathcal{R}\left(0, t^{*}\right)} r^{-2}\left|\psi_{k}\right|^{2}\right)^{-1} \int_{t=0} J_{\mu}^{N}(\psi) n^{\mu}
$$

Notice that all the previous quantities (7.39), (7.40) and (7.41) are finite, since, in view of the fact that the initial data for $\psi$ on $\{t=0\}$ were assumed to be supported in a set of the form $\left\{r \leq R_{\text {sup }}\right\}, \psi_{t^{*}}$ and $\psi_{k}, \psi_{\leq \omega_{+}}, \psi_{\geq \omega_{+}}$are all supported in the cylinder $\left\{r \lesssim R_{\text {sup }}+t^{*}\right\}^{31}$

Under the above normalizations, we compute that

$$
\int_{R\left(0, t^{*}\right)}(1+r)^{-2}\left|\tilde{\Psi}_{k}\right|^{2}=1,
$$

and Lemma 4.3 implies that for any $q \in \mathbb{N}$ :

$$
\int_{\mathcal{R}\left(0, t^{*}\right)} r^{q} \tilde{F}_{k} \leq C_{q}\left(\omega_{0}\right) \cdot \mathfrak{D} .
$$

Moreover, in case $\mathfrak{D} \geq 1$, the desired integrated local energy decay statement (7.38) for $\psi_{k}$ would readily follow: In this case, $\mathfrak{D} \geq 1$ immediately yields

$$
\int_{\mathcal{R}\left(0, t^{*}\right)}(1+r)^{-2}\left|\psi_{k}\right|^{2} \leq \int_{t=0} J_{\mu}^{N}(\psi) n^{\mu} .
$$

Using the Lagrangean inequality

$$
\begin{aligned}
\int_{\mathcal{R}\left(0, t^{*}\right)} \chi \cdot J_{\mu}^{N}\left(\psi_{k}\right) n^{\mu} \leq & C\left(\omega_{0}, \chi\right) \cdot\left(1+\omega_{k}^{2}\right) \cdot \int_{\mathcal{R}\left(0, t^{*}\right) \cap \operatorname{supp}(\chi)}\left|\psi_{k}\right|^{2} \\
& +C\left(\omega_{0}, \chi\right) \cdot \int_{t=0} J_{\mu}^{N}(\Psi) n^{\mu},
\end{aligned}
$$

which holds for any compactly supported cut-off $\chi$ and is easily obtained by using the current $J_{\mu}=\chi \cdot \operatorname{Re}\left\{\psi_{k} \cdot \partial_{\mu} \bar{\psi}_{k}\right\}-\left(\partial_{\mu} \chi\right) \cdot\left|\psi_{k}\right|^{2}$ (similarly with the extraction of (7.21)), as well as Lemmas 4.3, 4.4 and 4.8 (the latter enabling us to bound any $\left|T \psi_{k}\right|^{2}$ bulk terms by $\omega_{k}^{2}\left|\psi_{k}\right|^{2}$ terms), one easily infers (7.38). Therefore, we will assume without loss of generality that

$$
\mathfrak{D}<1 \text {. }
$$

We will also set

$$
\tilde{\Psi}_{k}=D \cdot \tilde{\Psi}_{k}
$$

\footnotetext{
31 Of course, as we remarked in Section 4.1, no constant in what follows will be allowed to depend on $R_{\text {sup }}, t^{*}$.
} 
where

$$
D \doteq\left(\sqrt{-\operatorname{det}(g)} \cdot g^{r r}\right)^{\frac{1}{2}}=r^{\frac{d-1}{2}}\left(1+O_{4}\left(r^{-1}\right)\right) .
$$

The factor $D$ was chosen so that $\tilde{\Psi}_{k}$ satisfies in the region $\left\{r \geq C_{1}\right\}$ an equation of the form

$$
\begin{aligned}
D \cdot \tilde{F}_{k}= & -\left(1+a_{t t}\right) \partial_{t}^{2} \tilde{\Psi}_{k}+\left(1+a_{r r}\right) \partial_{r}^{2} \tilde{\Psi}_{k} \\
& +r^{-2} \cdot\left(\Delta_{g_{\mathbb{S} d-1}+O_{4}\left(r^{-1-a}\right)}-\frac{(d-1)(d-3)}{4}\right) \tilde{\Psi}_{k} \\
& +a_{t \sigma} \cdot \partial_{t} \partial_{\sigma} \tilde{\Psi}_{k}+a_{\sigma} \cdot \partial_{\sigma} \tilde{\Psi}_{k}+a_{t} \cdot \partial_{t} \tilde{\Psi}_{k}+a \cdot \tilde{\Psi}_{k},
\end{aligned}
$$

where

- $a_{t t}=\frac{2 M}{r}+O_{4}\left(r^{-1-a}\right)$,

- $a_{r r}=-\frac{2 M}{r}+O_{4}\left(r^{-1-a}\right)$,

- $a_{t \sigma}=O_{4}\left(r^{-2-a}\right)$,

- $a_{\sigma}=O_{3}\left(r^{-3-a}\right)$,

- $a_{t}=O_{3}\left(r^{-1-a}\right)$ and

- $a=O_{2}\left(r^{-2-a}\right)$.

Note that the $\partial_{r}$ derivatives appear only in the $\partial_{r}^{2} \tilde{\Psi}_{k}$ term in this expression (owing to the normal form of the metric (2.4)).

Let us define for $r \geq C_{1}$ the following spherical energies (see [58]):

- $\mathscr{M}(\rho) \doteq \int_{\{r=\rho\} \cap \mathcal{R}\left(0, t^{*}\right)}\left|\tilde{\Psi}_{k}\right|^{2} d g_{\mathbb{S}^{d-1}} d t$.

- $\mathscr{F}(\rho) \doteq \int_{\{r=\rho\} \cap \mathcal{R}\left(0, t^{*}\right)} \tilde{\Psi}_{k} \cdot \partial_{r} \tilde{\Psi}_{k} d g_{\mathbb{S} d-1} d t$.

- $\mathscr{R}(\rho) \doteq \int_{\{r=\rho\} \cap \mathcal{R}\left(0, t^{*}\right)}\left|\partial_{r} \tilde{\Psi}_{k}\right|^{2} d g_{\mathbb{S} d-1} d t$.

- $\mathscr{A}(\rho) \doteq \int_{\{r=\rho\} \cap \mathcal{R}\left(0, t^{*}\right)}\left|\frac{1}{r} \partial_{\sigma} \tilde{\Psi}_{k}\right|^{2} d g_{\mathbb{S} d-1} d t$.

Notice that the measure of integration used is not the geometric one, but rather the "coordinate" one.

We will also need the following Pohozaev-type flux $\mathscr{P}$, which is defined as

$$
\begin{aligned}
\mathscr{P}(\rho)= & \int_{\{r=\rho\} \cap \mathcal{R}\left(0, t^{*}\right\}}\left\{\left|\partial_{r} \tilde{\Psi}_{k}\right|^{2}+\left(1+a_{t t}\right)\left(1+a_{r r}\right)^{-1}\left|\partial_{t} \tilde{\Psi}_{k}\right|^{2}\right. \\
& -\left(1+a_{r r}\right)^{-1} r^{-2}\left(\left|\partial_{\sigma} \tilde{\Psi}_{k}\right|^{2}+\frac{(d-1)(d-3)}{4}\left|\tilde{\Psi}_{k}\right|^{2}\right)+ \\
& -\left(1+a_{r r}\right)^{-1} a_{t \sigma} \partial_{t} \tilde{\Psi}_{k} \partial_{\sigma} \tilde{\Psi}_{k}+\left(\left(1+a_{r r}\right)^{-1} a_{\sigma}\right. \\
& \left.\left.-r^{-2} \partial_{\sigma}\left(1+a_{r r}\right)^{-1}\right) \cdot \tilde{\Psi}_{k} \partial_{\sigma} \tilde{\Psi}_{k}+a_{t} \tilde{\Psi}_{k} \partial_{t} \tilde{\Psi}_{k}+a \cdot\left|\tilde{\Psi}_{k}\right|^{2}\right\} d g_{\mathbb{S}^{d-1}} d t
\end{aligned}
$$

The above integrand can be obtained from (7.47) by applying multiplying the right hand side of equation (7.47) with $\left(1+a_{r r}\right)^{-1} \tilde{\Psi}_{k}$ and then formally integrating by parts in all second order terms schematically as: $a \cdot \partial^{2} \tilde{\Psi}_{k} \cdot \tilde{\Psi}_{k} \rightarrow-\partial \tilde{\Psi}_{k} \cdot \partial\left(a \cdot \tilde{\Psi}_{k}\right)$, after first multiplying the the $\partial_{r}^{2}$ term with -1 . 
Notice that if $C_{1} \gg 1$, then

$$
\mathscr{P}+2 \mathscr{A}+r^{-2-a} \cdot \mathscr{M} \geq c \cdot \int_{\{r=\rho\} \cap \mathcal{R}\left(0, t^{*}\right)}\left(\left|\partial_{t} \tilde{\Psi}_{k}\right|^{2}+\left|\partial_{r} \tilde{\Psi}_{k}\right|^{2}+\left|r^{-1} \partial_{\sigma} \tilde{\Psi}_{k}\right|^{2}\right) d g_{\mathbb{S}^{d-1}} d t .
$$

We will denote with $G$ any function $G:[0,+\infty) \rightarrow \mathbb{R}$ defined in terms of $\tilde{\Psi}_{k}$, such that there exist $r_{1} \geq 0$ and $C\left(\omega_{0}, R\right)>0$ for which we can bound

$$
\int_{r_{1}}^{\infty}|G(r)| d r \leq C\left(\omega_{0}, R\right) \cdot \mathfrak{D}
$$

with the constant $C\left(\omega_{0}, R\right)$ depending only on $\omega_{0}, R$ (and thus independent of $\psi, \psi_{k}, t^{*}$ etc.)

It readily follows from the definitions that

$$
\mathscr{M}, \mathscr{A}, \mathscr{R} \geq 0 \text {. }
$$

Furthermore, using a Cauchy-Schwarz inequality, we can bound:

$$
|\mathscr{F}| \leq(\mathscr{M})^{1 / 2} \cdot(\mathscr{R})^{1 / 2}
$$

Finally, since $\psi_{k}$ is supported in the cylinder $\left\{r \lesssim R_{\text {sup }}+t^{*}\right\}$, we have:

$$
\lim _{r \rightarrow \infty} \frac{1}{r} \int_{r}^{2 r}\{\mathscr{M}(\rho)+\mathscr{R}(\rho)+\mathscr{A}(\rho)+|\mathscr{P}(\rho)|\} d \rho=0 .
$$

The main result of this section (i.e. (7.38)) is a consequence of the fact that the spherical energies obey a particular system of equations of motion (see also [58]):

Lemma 7.3 The spherical energies satisfy the following system of ODE's:

$$
\left\{\begin{array}{l}
\frac{d}{d r} \mathscr{M}=2 \mathscr{F} \\
\frac{d}{d r} \mathscr{F}=2 \mathscr{R}-\mathscr{P}+G+O\left(r^{-3}\right) \cdot \mathscr{M} \\
\frac{d}{d r} \mathscr{P}=\frac{2}{r} \mathscr{A}+O\left(r^{-3-a}\right) \cdot \mathscr{M}+O\left(r^{-1-a}\right)\{\mathscr{P}+2 \mathscr{A}\}+G .
\end{array}\right.
$$

The constants in the Big $O$ notation are allowed to depend on $\omega_{0}, R$.

Proof The first equation of (7.54) follows readily due to the particular choice of measure on $\{r=$ const $\} \cap \mathcal{R}\left(0, t^{*}\right)$ slices, which makes it invariant under the flow of $\partial_{r}$. Namely,

$$
\frac{d}{d \rho}\left(\int_{\{r=\rho\} \cap \mathcal{R}\left(0, t^{*}\right)}\left|\tilde{\Psi}_{k}\right|^{2} d g_{\mathbb{S}^{d-1}} d t\right)=2 \int_{\{r=\rho\} \cap \mathcal{R}\left(0, t^{*}\right)} \partial_{r} \tilde{\Psi}_{k} \cdot \tilde{\Psi}_{k} d g_{\mathbb{S}^{d-1}} d t .
$$

In order to extract the second equation of (7.54), we must use the fact that $\tilde{\Psi}_{k}$ satisfies equation (7.47). In particular, we compute 


$$
\begin{aligned}
& \frac{d}{d \rho}\left(\int_{\{r=\rho\} \cap \mathcal{R}\left(0, t^{*}\right)} \partial_{r} \tilde{\Psi}_{k} \cdot \tilde{\Psi}_{k} d g_{\mathbb{S} d-1} d t\right) \\
& =\int_{\{r=\rho\} \cap \mathcal{R}\left(0, t^{*}\right)}\left(\left|\partial_{r} \tilde{\Psi}_{k}\right|^{2}+\tilde{\Psi}_{k} \cdot \partial_{r}^{2} \tilde{\Psi}_{k}\right) d g_{\mathbb{S} d-1} d t .
\end{aligned}
$$

Since we can use equation (7.47) to replace $\partial_{r}^{2} \tilde{\Psi}_{k}$ with

$$
\begin{aligned}
\left(1+a_{r r}\right)^{-1}\{ & D \cdot \tilde{F}_{k}+\left(1+a_{t t}\right) \partial_{t}^{2} \tilde{\Psi}_{k}-r^{-2}\left(\Delta_{g_{\mathbb{S}} d-1}+O_{4}\left(r^{-1-a}\right)\right. \\
& \left.-\frac{(d-1)(d-3)}{4} \tilde{\Psi}_{k}\right) \\
& \left.-a_{t \sigma} \partial_{t} \partial_{\sigma} \tilde{\Psi}_{k}-a_{\sigma} \partial_{\sigma} \tilde{\Psi}_{k}-a_{t} \partial_{t} \tilde{\Psi}_{k}-a \cdot \tilde{\Psi}_{k}\right\}
\end{aligned}
$$

we calculate from (7.56) that:

$$
\begin{aligned}
\frac{d}{d \rho} & \left(\int_{\{r=\rho\} \cap \mathcal{R}\left(0, t^{*}\right)} \partial_{r} \tilde{\Psi}_{k} \cdot \tilde{\Psi}_{k} d g_{\mathbb{S}^{d-1}} d t\right) \\
= & \int_{\{r=\rho\} \cap \mathcal{R}\left(0, t^{*}\right)}\left(\left|\partial_{r} \tilde{\Psi}_{k}\right|^{2}+\left(1+a_{r r}\right)^{-1} \tilde{\Psi}_{k} \cdot\left\{D \cdot \tilde{F}_{k}+\left(1+a_{t t}\right) \partial_{t}^{2} \tilde{\Psi}_{k}\right.\right. \\
& -r^{-2}\left(\Delta_{g_{\mathbb{S}} d-1}+O_{4}\left(r^{-1-a}\right)\right. \\
& \left.\left.\left.-a_{t} \partial_{t} \tilde{\Psi}_{k}-\frac{(d-1)(d-3)}{4} \tilde{\Psi}_{k}\right)-a_{t \sigma} \partial_{t} \partial_{\sigma} \tilde{\Psi}_{k}\right\}\right) d g_{\mathbb{S}^{d-1}} d t .
\end{aligned}
$$

Hence, after integrating by parts in $t$ and $\sigma$ in order to we eliminate the second order derivatives, we conclude in view of (7.48) Lemma $4.4^{32}$ that:

$$
\begin{aligned}
& \frac{d}{d \rho}\left(\int_{\{r=\rho\} \cap \mathcal{R}\left(0, t^{*}\right)} \partial_{r} \tilde{\Psi}_{k} \cdot \tilde{\Psi}_{k} d g_{\mathbb{S}^{d-1}} d t\right) \\
& \quad=2 \mathscr{R}-\mathscr{P}+G+\int_{\{r=\rho\} \cap \mathcal{R}\left(0, t^{*}\right)} D \cdot\left|\tilde{F}_{k}\right| \cdot\left|\tilde{\Psi}_{k}\right| d g_{\mathbb{S}^{d-1}} d t .
\end{aligned}
$$

The second equation of (7.54) now follows from (7.59), after applying a CauchySchwarz inequality for the last term of the right hand side and using Lemma 4.3 to show that $\int_{\{r=\rho\} \cap R\left(0, t^{*}\right)} r^{3} D^{2}\left|\tilde{F}_{k}\right|^{2} d g_{\mathbb{S}^{d-1}} d t$ is a $G$-type function in $\rho$.

For the extraction of the third equation of (7.54), we work in a similar way. We calculate from the expression $(7.48)$ for $\mathscr{P}(\rho)$ that

\footnotetext{
32 Which shows with the boundary terms that result from integration by parts in $t$ sum up to a $G$-type function of $r$.
} 


$$
\begin{aligned}
\frac{d}{d \rho} \mathscr{P}(\rho)= & \int_{\{r=\rho\} \cap \mathcal{R}\left(0, t^{*}\right\}} 2 r^{-3}\left(1+O\left(r^{-1}\right)\right)\left(\left|\partial_{\sigma} \tilde{\Psi}_{k}\right|^{2}+\frac{(d-1)(d-3)}{4}\left|\tilde{\Psi}_{k}\right|^{2}\right) d g_{\mathbb{S}^{2}} d t \\
& +\int_{\{r=\rho\} \cap \mathcal{R}\left(0, t^{*}\right\}}\left\{2 \partial_{r}^{2} \tilde{\Psi}_{k} \cdot \partial_{r} \tilde{\Psi}_{k}+2\left(1+a_{t t}\right)\left(1+a_{r r}\right)^{-1} \partial_{r} \partial_{t} \tilde{\Psi}_{k} \cdot \partial_{t} \tilde{\Psi}_{k}\right. \\
& -2\left(1+a_{r r}\right)^{-1} r^{-2}\left(g_{\mathbb{S}^{d-1}}\left(\nabla^{\mathbb{S}^{d-1}} \partial_{r} \tilde{\Psi}_{k}, \nabla^{\mathbb{S}^{d-1}} \tilde{\Psi}_{k}\right)+\frac{(d-1)(d-3)}{4} \partial_{r} \tilde{\Psi}_{k} \cdot \tilde{\Psi}_{k}\right) \\
& -\left(1+a_{r r}\right)^{-1} a_{t \sigma} \partial_{r} \partial_{t} \tilde{\Psi}_{k} \cdot \partial_{\sigma} \tilde{\Psi}_{k}-\left(1+a_{r r}\right)^{-1} a_{t \sigma} \partial_{t} \tilde{\Psi}_{k} \cdot \partial_{r} \partial_{\sigma} \tilde{\Psi}_{k} \\
& +\left(\left(1+a_{r r}\right)^{-1} a_{\sigma}-r^{-2} \partial_{\sigma}\left(1+a_{r r}\right)^{-1}\right) \tilde{\Psi}_{k} \cdot \partial_{r} \partial_{\sigma} \tilde{\Psi}_{k}+a_{t} \tilde{\Psi}_{k} \cdot \partial_{r} \partial_{t} \tilde{\Psi}_{k} \\
& +O\left(r^{-3-a}\right) \partial_{r} \tilde{\Psi}_{k} \cdot \partial_{\sigma} \tilde{\Psi}_{k}+O\left(r^{-1-a}\right) \partial_{r} \tilde{\Psi}_{k} \cdot \partial_{t} \tilde{\Psi}_{k}+O\left(r^{-2-a}\right) \partial_{r} \tilde{\Psi}_{k} \cdot \tilde{\Psi}_{k} \\
& +O\left(r^{-2-a}\right)\left|\partial_{t} \tilde{\Psi}_{k}\right|^{2}+O\left(r^{-3-a}\right) \partial_{t} \tilde{\Psi}_{k} \cdot \partial_{\sigma} \tilde{\Psi}_{k}+O\left(r^{-4-a}\right) \tilde{\Psi}_{k} \cdot \partial_{\sigma} \tilde{\Psi}_{k} \\
& \left.+O\left(r^{-2-a}\right) \tilde{\Psi}_{k} \cdot \partial_{t} \tilde{\Psi}_{k}+O\left(r^{-3-a}\right)\left|\tilde{\Psi}_{k}\right|^{2}\right\} d g_{\mathbb{S}^{d-1}} d t .
\end{aligned}
$$

Using equation (7.47) to replace $\partial_{r}^{2} \tilde{\Psi}_{k}$ with (7.57) in (7.60) and then integrating by parts in $t$ and $\sigma$ in the highest order terms, then all the second order derivatives of $\tilde{\Psi}_{k}$ and $\tilde{\Psi}_{k}$ in (7.60) cancel out. Thus, the third equation of (7.54) readily follows, after, of course, treating the boundary terms resulting from the integration by parts, as well as the $\tilde{F}_{k}$ terms, using Lemmas 4.4 and 4.3.

Lemma 7.4 The Pohozaev flux $\mathscr{P}$ satisfies in $\left\{r \geq C_{1}\right\}$ for $C_{1}$ suitably large:

$$
\mathscr{P} \lesssim \mathfrak{D}+O\left(r^{-2-a}\right) \cdot \mathscr{M}
$$

Proof If we set $\mathscr{P}^{*} \doteq \mathscr{P}-C_{0} r^{-2-a} \mathscr{M}$, for a positive constant $C_{0}$ to be determined, we calculate from (7.54):

$$
\begin{aligned}
\frac{d}{d r} \mathscr{P}^{*}= & \frac{2}{r} \mathscr{A}+O\left(r^{-3-a}\right) \mathscr{M}+O\left(r^{-1-a}\right)\left\{\mathscr{P}^{*}+C_{0} r^{-2-a} \mathscr{M}+2 \mathscr{A}\right\}+G \\
& +(2+a) C_{0} r^{-3-a} \mathscr{M}-2 C_{0} r^{-2-a} \mathscr{F}+C_{0} O\left(r^{-4-2 a}\right) \mathscr{M}
\end{aligned}
$$

By bounding

$$
|\mathscr{F}| \leq r^{-1} \mathscr{M}+r \mathscr{R} \leq r^{-1}\left(1+C_{0} \cdot O\left(r^{-a}\right)\right) \mathscr{M}+C r\left(1+O\left(r^{-a}\right)\right) \mathscr{A}+C r \mathscr{P}^{*}
$$

and substituting in (7.63), we obtain

$$
\begin{aligned}
& \frac{d}{d r} \mathscr{P}^{*} \geq \frac{2}{r}\left\{1+O\left(r^{-a}\right)-C_{0}\left(r^{-a}+O\left(r^{-2 a}\right)\right)\right\} \mathscr{A} \\
& \quad+\left(C_{0} r^{-3-a}\left(1+O\left(r^{-1}\right)\right)+O\left(r^{-3-a}\right)+C_{0} \cdot O\left(r^{-3-2 a}\right)\right) \mathscr{M} \\
& \quad+C_{0} \cdot O\left(r^{-1-a}\right) \mathscr{P}^{*}+G .
\end{aligned}
$$


Hence, if $C_{0}$ is chosen sufficiently large, and $C_{1}$ (and thus $r$ ) is large enough, both in terms of the geometry of $(\mathcal{D}, g)$, the coefficients in front of the $\mathscr{M}$ and $\mathscr{A}$ terms in (7.65) will be positive, resulting in the inequality

$$
\frac{d}{d r} \mathscr{P}^{*} \geq O\left(r^{-1-a}\right) \mathscr{P}^{*}+G
$$

from which (7.62) follows by an application of Gronwall's inequality (in view of the initial conditions (7.53) at infinity, as well as the definition of $\mathscr{P}^{*}$ ).

In view of Lemma 4.7 (notice that we can allow $R \rightarrow \infty$ in the statement of that lemma, since at this point we only care about the left hand side of (4.70) which is independent of $R$ ), we can bound from below

$$
\int_{\{r=\rho\} \cap \mathcal{R}\left(0, t^{*}\right)}\left|\partial_{t} \tilde{\Psi}_{k}\right|^{2} d g_{\mathbb{S}^{2}} d t \geq c \cdot \omega_{k-1}^{2} \mathscr{M}+G .
$$

Hence, from (7.48) we can bound from below:

$$
\mathscr{A} \geq\left(1+O\left(r^{-1}\right)\right)(\mathscr{R}-\mathscr{P})+c \cdot \omega_{k-1}^{2} \mathscr{M}+O\left(r^{-2-a}\right) \mathscr{M} .
$$

Moreover, in view of (7.52), we can eliminate the quantity $\mathscr{R}$ from (7.54) through the estimate

$$
\mathscr{R} \geq \frac{\mathscr{F}^{2}}{\mathscr{M}} .
$$

Hence, substituting (7.68) and (7.69) in (7.54), we obtain the following system of differential inequalities:

$$
\left\{\begin{array}{l}
\frac{d}{d r} \mathscr{M}=2 \mathscr{F} \\
\frac{d}{d r} \mathscr{F} \geq 2 \frac{\mathscr{F}^{2}}{\mathscr{M}}-\mathscr{P}+G+O\left(r^{-3}\right) \mathscr{M} \\
\frac{d}{d r} \mathscr{P} \geq \frac{2}{r}\left(1+O\left(r^{-1}\right)\right) \cdot\left(\frac{\mathscr{F}^{2}}{\mathscr{M}}-\mathscr{P}+c \cdot \omega_{k-1}^{2} \mathscr{M}\right)+O\left(r^{-3-a}\right) \mathscr{M}+O\left(r^{-1-a}\right) \mathscr{P}+G .
\end{array}\right.
$$

Note that in the third equation of (7.70), the last term of the right hand side can also be replaced for free by $O\left(r^{-1}+\omega_{k-1}\right) \cdot G$, since we have $\omega_{0} \leq \omega_{k-1}$ and we allow the constants in the big $O$ notation to depend on $\omega_{0}$. Therefore, we see that we can immediately apply on the above system of differential inequalities the ODE lemma from Section 10 in [58], which can be stated as follows:

Lemma 7.5 (Rodnianski-Tao) Let $\mathscr{M}, \mathscr{F}, \mathscr{P}:\left[C_{1},+\infty\right) \rightarrow(0,+\infty)$ satisfy (7.53), (7.62) and (7.70). Then, provided $C_{1}$ is large enough (independently of $\omega_{k-1}$ ), for any positive constant $C_{2} \gg C_{1}$ (again with magnitude independent of $\omega_{k-1}$ ) there exists another positive constant $C\left(C_{1}, C_{2}\right)$ such that one of the following scenarios holds:

1. Boundedness Scenario: There exists an $R_{0}$ with $C_{1}<R_{0}<C\left(C_{1}, C_{2}\right)$ such that

$$
\mathscr{M}(r) \leq C\left(C_{1}, C_{2}\right)\left(\omega_{k-1}^{-2 C\left(C_{1}, C_{2}\right)}+1\right) \mathfrak{D}
$$

for all $r \in\left[\frac{1}{2} R_{0}, 4 R_{0}\right]$ 
2. Exponential growth from infinity Scenario: For all $C_{1} \leq r \leq 10 C_{1}$ we can bound

$$
\frac{d}{d r} \mathscr{M}(r) \leq-C_{2}\left(1+\omega_{k-1}\right) \mathscr{M}(r) .
$$

Using Lemma 7.5 we will suitably absorb the first term of the right hand side of (7.1), thus obtaining the desired integrated local energy decay statement (7.38).

We first fix $C_{1}=R$, assuming of course without loss of generality that $R$ was large enough in terms of the geometry of $(\mathcal{D}, g)$. We then choose $C_{2}=C_{2}\left(C_{1}, \omega_{0}\right)$ large enough in terms of $C_{1}$ and $\omega_{0}$ so that

$$
C_{2} \gg \omega_{0}, R C\left(\omega_{0}, R\right) \cdot \sup _{C_{1} \leq r \leq 10 C_{1}} w
$$

where $C\left(\omega_{0}, R\right)$ is the constant appearing in the $s$ parameter in inequality (7.1). In this way, we can also bound (since $s=C \cdot \omega_{k}$ in $(7.1)$, and $\omega_{k} \leq \omega_{k-1}+1$ ):

$$
C_{2} \cdot\left(1+\omega_{k-1}\right)-s \cdot \sup _{C_{1} \leq r \leq 10 C_{1}} w \gg \omega_{0}, R \omega_{k-1}
$$

In view of Lemma 7.5 , there are only two possible scenarios for $\mathscr{M}$ :

Case 1. Assume that the boundedness scenario in Lemma 7.5 holds, that is (7.71) is true. From (7.1) we obtain (replacing $R$ in (7.1) by the value $R_{0}$ which is provided by the boundedness scenario):

$$
\begin{aligned}
& \int_{\mathcal{R}\left(0, t^{*}\right) \cap\left\{r \leq R_{0}\right\}}\left(e^{2 s w}+e^{2 s w^{\prime}}\right) \cdot\left(J_{\mu}^{N}\left(\psi_{k}\right) n^{\mu}+\left|\psi_{k}\right|^{2}\right) \\
& \leq C\left(\omega_{0}, R_{0}\right) \int_{\left.\mathcal{R}\left(0, t^{*}\right) \cap\left\{R_{0} \leq r \leq 2 R_{0}\right\}\right)}\left(1+s^{2}\right) e^{2 s w}\left\{J_{\mu}^{N}\left(\psi_{k}\right) n^{\mu}+\left|\psi_{k}\right|^{2}\right\} \\
& \quad+e^{C\left(R_{0}\right) \cdot s} \cdot C\left(\omega_{0}, R_{0}\right) \int_{t=0} J_{\mu}^{N}(\psi) n^{\mu}
\end{aligned}
$$

Furthermore, applying (as we did earlier) the divergence identity for the current $J_{\mu}=\chi \cdot \psi_{k} \cdot \partial_{\mu} \psi_{k}-\left(\partial_{\mu} \chi\right) \cdot\left|\psi_{k}\right|^{2}$, combined with Lemmas 4.3 and 4.4, as well the bounded frequency estimate 4.8 (serving to bound $\left|\partial_{t} \psi_{k}\right|^{2}$ bulk terms by $\omega_{k}^{2}\left|\psi_{k}\right|^{2}$ terms), we obtain the following Lagrangean inequality:

$$
\begin{aligned}
& \int_{\mathcal{R}\left(0, t^{*}\right)} \chi \cdot J_{\mu}^{N}\left(\Psi_{k}\right) n^{\mu} \leq C\left(\omega_{0}, \chi\right) \cdot\left(1+\omega_{k}^{2}\right) \cdot \int_{\mathcal{R}\left(0, t^{*}\right) \cap \operatorname{supp}(\mathrm{\chi})}\left|\Psi_{k}\right|^{2} \\
& \quad+C\left(\omega_{0}, \chi\right) \cdot \int_{t=0} J_{\mu}^{N}(\psi) n^{\mu}
\end{aligned}
$$

Therefore, using (7.76) for a cut off $\chi$ supported in $\left\{\frac{1}{2} R_{0} \leq r \leq 4 R_{0}\right\}$ and being equal to 1 in $\left\{R_{0} \leq r \leq 2 R_{0}\right\}$, combined with (7.71), we can readily bound: 


$$
\begin{aligned}
& \int_{\left.\mathcal{R}\left(0, t^{*}\right) \cap\left\{R_{0} \leq r \leq 2 R_{0}\right\}\right)}\left(1+s^{2}\right) e^{2 s w}\left\{J_{\mu}^{N}\left(\psi_{k}\right) n^{\mu}+\left|\psi_{k}\right|^{2}\right\} \\
& \leq C\left(R, \omega_{0}\right) e^{C\left(R, \omega_{0}\right) \omega_{+}} \int_{t=0} J_{\mu}^{N}(\psi) n^{\mu}
\end{aligned}
$$

Hence, since $R=C_{1}<R_{0}<C_{2}\left(R, \omega_{0}\right)$, in this case we can estimate from (7.75) and (7.77):

$$
\int_{\{r \leq R\} \cap \mathcal{R}\left(0, t^{*}\right)}\left\{J_{\mu}^{N}\left(\psi_{k}\right) n^{\mu}+\left|\psi_{k}\right|^{2}\right\} \leq C\left(R, \omega_{0}\right) e^{C\left(R, \omega_{0}\right) \omega_{+}} \int_{t=0} J_{\mu}^{N}(\psi) n^{\mu} .
$$

Case 2. Assume that the exponential growth scenario in Lemma 7.5 holds, that is (7.72) is true. Then a simple application of Gronwall's inequality on (7.72) yields:

$\int_{\left\{5 C_{1} \leq r \leq 10 C_{1}\right\}} \mathscr{M}(r) d r \leq C_{3} \cdot e^{-C_{2}\left(1+\omega_{k-1}\right)} \int_{\left\{2 C_{1} \leq r \leq 4 C_{1}\right\}} \mathscr{M}(r) d r$.

Applying (7.1) (for $6 C_{1}$ in place of $R$ ), we obtain

$$
\begin{aligned}
& \int_{\mathcal{R}\left(0, t^{*}\right) \cap\left\{r \leq 6 C_{1}\right\}}\left(e^{2 s w}+e^{2 s w^{\prime}}\right) \cdot\left(J_{\mu}^{N}\left(\psi_{k}\right) n^{\mu}+\left|\psi_{k}\right|^{2}\right) \\
& \leq C\left(\omega_{0}, R\right) \int_{\left.\mathcal{R}\left(0, t^{*}\right) \cap\left\{6 C_{1} \leq r \leq 7 C_{1}\right\}\right)}\left(1+s^{2}\right) e^{2 s w}\left(J_{\mu}^{N}\left(\psi_{k}\right) n^{\mu}+\left|\psi_{k}\right|^{2}\right)+ \\
& \quad+e^{C\left(C_{1}\right) \cdot s} \cdot C\left(\omega_{0}, R\right) \int_{t=0} J_{\mu}^{N}(\psi) n^{\mu} .
\end{aligned}
$$

In view of (7.76) applied for a cut off $\chi$ supported in $5 C_{1} \leq r \leq 8 C_{1}$ and being equal to 1 in $6 C_{1} \leq r \leq 7 C_{1}$, we can also bound

$$
\begin{aligned}
& \int_{\left.\mathcal{R}\left(0, t^{*}\right) \cap\left\{6 C_{1} \leq r \leq 7 C_{1}\right\}\right)}\left(1+s^{2}\right) e^{2 s w}\left(J_{\mu}^{N}\left(\psi_{k}\right) n^{\mu}+\left|\psi_{k}\right|^{2}\right) \\
& \leq C\left(R, \omega_{0}\right)\left(1+\omega_{k}^{2}\right) e^{s \cdot \sup _{C_{1} \leq r \leq 10 C_{1}} w} \int_{\left\{5 C_{1} \leq r \leq 8 C_{1}\right\}} \mathscr{M}(r) d r+ \\
& \quad+C\left(R, \omega_{0}\right) e^{C\left(R, \omega_{0}\right) \omega_{k}} \int_{t=0} J_{\mu}^{N}(\psi) n^{\mu} .
\end{aligned}
$$

From (7.81) and (7.79)we infer:

$$
\begin{aligned}
& \int_{\left.\mathcal{R}\left(0, t^{*}\right) \cap\left\{6 C_{1} \leq r \leq 7 C_{1}\right\}\right)}\left(1+s^{2}\right) e^{2 s w}\left(J_{\mu}^{N}\left(\psi_{k}\right) n^{\mu}+\left|\psi_{k}\right|^{2}\right) \\
& \leq C\left(R, \omega_{0}\right)\left(1+\omega_{k}^{2}\right) e^{s \cdot \sup _{C_{1} \leq r \leq 10 C_{1}} w-C_{2} \omega_{k-1}} \int_{\left\{2 C_{1} \leq r \leq 4 C_{1}\right\}} \mathscr{M}(r) d r \\
& \quad+C\left(R, \omega_{0}\right) e^{C\left(R, \omega_{0}\right) \omega_{k}} \int_{t=0} J_{\mu}^{N}(\psi) n^{\mu} .
\end{aligned}
$$


Therefore, since in view of (7.74) the first term of the right hand side of (7.82) can be absorbed into the left hand side of (7.80), we conclude from (7.80):

$$
\int_{\{r \leq R\} \cap \mathcal{R}\left(0, t^{*}\right)}\left\{J_{\mu}^{N}\left(\psi_{k}\right) n^{\mu}+\left|\psi_{k}\right|^{2}\right\} \leq C\left(R, \omega_{0}\right) e^{C\left(R, \omega_{0}\right) \omega_{+}} \int_{t=0} J_{\mu}^{N}(\psi) n^{\mu} .
$$

Thus, the proof of Proposition 7.2 is complete.

As a corollary of Propositions 7.2 and 6.1, we will obtain an integrated local energy decay statement for the $\psi_{\leq \omega_{+}}$component of $\psi$. This will be the final result of this section.

Corollary 7.6 For any $R>0$ there exists $a \delta=\delta(R)>0$, such that for any smooth solution $\psi$ to the wave equation on $J^{+}(\Sigma) \cap \mathcal{D}$ with compactly supported initial data on $\Sigma$, any $0<\omega_{0}<\delta$ and any $\omega_{+}>1$ we can bound:

$$
\int_{\{r \leq R\} \cap \mathcal{R}\left(0, t^{*}\right)}\left\{J_{\mu}^{N}\left(\psi_{\leq \omega_{+}}\right) n^{\mu}+\left|\psi_{\leq \omega_{+}}\right|^{2}\right\} \leq C\left(R, \omega_{0}\right) \cdot e^{C\left(R, \omega_{0}\right) \omega_{+}} \int_{t=0} J_{\mu}^{N}(\psi) n^{\mu}
$$

Proof Proposition 6.1 for the zero frequency part $\psi_{0}$ of $\psi$ provides an integrated local energy decay statement for $\psi_{0}$ :

$$
\int_{\{r \leq R\} \cap \mathcal{R}\left(0, t^{*}\right)}\left(J_{\mu}^{N}\left(\psi_{0}\right) n^{\mu}+\left|\psi_{0}\right|^{2}\right) \leq C\left(R, \omega_{0}\right) \cdot \int_{t=0} J_{\mu}^{N}(\psi) n^{\mu}
$$

Adding (6.1) and (7.38) for all $1 \leq|k| \leq n$, and recalling that $n \sim \frac{\omega_{+}}{\omega_{0}}$, we obtain:

$$
\begin{aligned}
& \sum_{k=-n}^{n}\left\{\int_{\{r \leq R\} \cap \mathcal{R}\left(0, t^{*}\right)}\left(J_{\mu}^{N}\left(\psi_{k}\right) n^{\mu}+\left|\psi_{k}\right|^{2}\right)\right\} \\
& \leq C\left(R, \omega_{0}\right) \cdot \omega_{+} \cdot e^{C\left(R, \omega_{0}\right) \cdot \omega_{+}} \int_{t=0} J_{\mu}^{N}(\psi) n^{\mu}
\end{aligned}
$$

The result now follows readily from the fact that $\psi_{\leq \omega_{+}}=\sum_{k=-n}^{n} \psi_{k}$ :

$$
\begin{aligned}
& \int_{\{r \leq R\} \cap \mathcal{R}\left(0, t^{*}\right)}\left(J_{\mu}^{N}\left(\Psi_{\leq \omega_{+}}\right) n^{\mu}+\left|\psi_{\leq \omega_{+}}\right|^{2}\right) \\
& \quad \leq \int_{\{r \leq R\} \cap \mathcal{R}\left(0, t^{*}\right)} 2 n \cdot \sum_{k=-n}^{n}\left(J_{\mu}^{N}\left(\Psi_{k}\right) n^{\mu}+\left|\psi_{k}\right|^{2}\right) \\
& \leq C\left(R, \omega_{0}\right) \cdot \omega_{+}^{2} \cdot e^{C\left(R, \omega_{0}\right) \cdot \omega_{+}} \int_{t=0} J_{\mu}^{N}(\psi) n^{\mu} \\
& \leq C\left(R, \omega_{0}\right) \cdot e^{C(R) \cdot \omega_{+}} \int_{t=0} J_{\mu}^{N}(\psi) n^{\mu} .
\end{aligned}
$$




\section{Polynomial Decay of the Local Energy of $\psi_{\leq \omega_{+}}$}

In this Section, we will establish a polynomial decay rate for the local energy of $\psi_{\leq \omega_{+}}$, using the integrated local energy decay estimate of Corollary 7.6, together with the generalisation of the $r^{p}$-weighted energy method of Dafermos and Rodnianski established in [52].

We will thus examine the energy of $\psi_{\leq \omega_{+}}$on a foliation of spacelike hypersurfaces terminating at null infinity. ${ }^{33}$ In the $r \gg 1$ region, such a foliation has been constructed in terms of the level sets of the function $\tilde{u}_{\eta^{\prime}}$, see (5.29). We will extend $\tilde{u}_{\eta^{\prime}}$ to a function defined on the whole of $\mathcal{D} \backslash \mathcal{H}^{-}$, with everywhere spacelike level sets, such that it coincides with the time function $t$ in the region $r \lesssim 1$ :

Definition For any fixed $\eta^{\prime} \in(0,1+2 a)$ and any constant $R_{3} \gg 1$ large in terms of the geometry of $(\mathcal{D}, g)$, we will define the function $\bar{t}: D \backslash \mathcal{H}^{-} \rightarrow \mathbb{R}$,

$$
\bar{t}=t+\chi_{R_{3}}(r) \cdot\left(\tilde{u}_{\eta^{\prime}}-t\right),
$$

where $u_{\eta^{\prime}}$ is defined as (5.29) and $\chi_{R_{3}}: \mathbb{R} \rightarrow[0,1]$ is a smooth cut-off function equal to 0 for $r \leq R_{3}$ and equal to 1 for $r \geq R_{3}+1$. We will also denote with $S_{\tau}$ the hyperboloids $\{\bar{t}=\tau\}$, which for $\left\{r \geq R_{3}\right\}$ coincide with $\tilde{S}_{\tau}$.

We will establish the following proposition:

Proposition 8.1 There exists a positive constant $C$, depending only on the geometry of $(\mathcal{D}, g)$, such that for any smooth solution $\psi$ to the wave equation on $J^{+}(\Sigma) \cap D$ with compactly supported initial data on $\Sigma$, any $0 \leq \delta_{0} \leq 1$, any $\omega_{+}>1$ and any $\tau \in\left[\frac{1}{4} t^{*}, \frac{3}{4} t^{*}\right]$ we can bound:

$$
\begin{gathered}
\int_{S_{\tau} \cap \mathcal{R}\left(0, t^{*}\right)} J_{\mu}^{N}\left(\psi_{\leq \omega_{+}}\right) n_{S_{\tau}}^{\mu} \leq \frac{C}{\left(\tau-\frac{1}{4} t^{*}\right)^{\delta_{0}}} \\
\cdot\left\{e^{C \omega_{+}} \int_{t=0} J_{\mu}^{N}(\psi) n^{\mu}+\int_{t=0}(1+r)^{\delta_{0}} J_{\mu}^{N}(\psi) n^{\mu}\right\} .
\end{gathered}
$$

Proof Let us fix $R=R_{3}$, assuming, of course, without loss of generality that $R_{3}$ has been chosen sufficiently large in terms of the geometry of $(\mathcal{D}, g)$. We will also fix a value $\omega_{0}=\omega_{0}(R)$, so that according to Corollary 7.6 we can estimate:

$$
\int_{\{r \leq R\} \cap \mathcal{R}\left(0, t^{*}\right)}\left\{J_{\mu}^{N}\left(\psi_{\leq \omega_{+}}\right) n^{\mu}+\left|\psi_{\leq \omega_{+}}\right|^{2}\right\} \leq C \cdot e^{C \omega_{+}} \int_{t=0} J_{\mu}^{N}(\psi) n^{\mu} .
$$

In view of (5.34), we can bound:

$$
\int_{0}^{\infty}\left\{\int_{S_{t} \cap \mathcal{R}\left(0, t^{*}\right) \cap\{r \geq R\}} J_{\mu}^{N}\left(\psi_{\leq \omega_{+}}\right) n_{S_{t}}^{\mu}\right\} d t
$$

\footnotetext{
33 Since the energy of $\psi_{\leq \omega_{+}}$on the $\Sigma_{t}$ hypersurfaces can not be expected to decay in $t$.
} 


$$
\leq C \int_{\{R-1 \leq r \leq R\} \cap \mathcal{R}\left(0, t^{*}\right)}\left(J_{\mu}^{N}\left(\psi_{\leq \omega_{+}}\right) n^{\mu}+\left|\psi_{\leq \omega_{+}}\right|^{2}\right)+C \int_{t=0}(1+r) J_{\mu}^{N}(\psi) n^{\mu},
$$

where $n_{S_{t}}$ is the future directed unit normal of the foliation $S_{t}$.

From (8.4) and (8.3) we thus conclude

$$
\int_{0}^{\infty}\left\{\int_{S_{\tau} \cap \mathcal{R}\left(0, t^{*}\right)} J_{\mu}^{N}\left(\psi_{\leq \omega_{+}}\right) n_{S_{\tau}}^{\mu}\right\} d \tau \leq C \cdot\left\{e^{C \omega_{+}} \int_{t=0} J_{\mu}^{N}(\psi) n^{\mu}+\int_{t=0} r J_{\mu}^{N}(\psi) n^{\mu}\right\} .
$$

In order to simplify the expressions in the next few lines, we will set:

$$
f(\tau) \doteq \int_{S_{\tau} \cap \mathcal{R}\left(0, t^{*}\right)} J_{\mu}^{N}\left(\psi_{\leq \omega_{+}}\right) n_{S_{\tau}}^{\mu}
$$

Then (8.5) reads

$$
\int_{0}^{\infty} f(\tau) d \tau \leq C \cdot\left\{e^{C \omega_{+}} \int_{t=0} J_{\mu}^{N}(\psi) n^{\mu}+\int_{t=0} r J_{\mu}^{N}(\psi) n^{\mu}\right\}
$$

Due to Lemma 4.6 for $q=2$, for any $\tau_{1} \leq \tau_{2}$ in the interval $\left[\frac{1}{4} t^{*}, \frac{3}{4} t^{*}\right.$ ], setting $t_{s t}=\frac{1}{4} t^{*}$ in (4.58) (notice that this implies that $t_{s t} \sim t_{1} \sim t_{2}-t_{1}$ ), the following boundedness statement holds for $f$ :

$$
f\left(\tau_{2}\right) \leq C \cdot f\left(\tau_{1}\right)+C \tau_{2}^{2} \int_{t=0} J_{\mu}^{N}(\psi) n^{\mu}
$$

Hence, we infer from (8.7) and (8.8) for any $\tau \in\left[\frac{1}{4} t^{*}, \frac{3}{4} t^{*}\right]$ :

$$
\begin{aligned}
C & \cdot\left\{e^{C \omega_{+}} \int_{t=0} J_{\mu}^{N}(\psi) n^{\mu}+\int_{t=0} r J_{\mu}^{N}(\psi) n^{\mu}\right\} \geq \int_{0}^{\infty} f(s) d s \geq \int_{\frac{1}{4} t^{*}}^{\tau} f(s) d s \\
& \geq \int_{\frac{1}{4} t^{*}}^{\tau}\left(\frac{f(\tau)}{C}-\frac{1}{\tau^{2}} \int_{t=0} J_{\mu}^{N}(\psi) n^{\mu}\right) d s \\
& \geq c \cdot\left(\tau-\frac{t^{*}}{4}\right) f(\tau)-C \cdot \int_{t=0} J_{\mu}^{N}(\psi) n^{\mu}
\end{aligned}
$$

From (8.9) it readily follows that forany $\tau \in\left[\frac{1}{4} t^{*}, \frac{3}{4} t^{*}\right]$ we can bound:

$$
\int_{S_{\tau} \cap \mathcal{R}\left(0, t^{*}\right)} J_{\mu}^{N}\left(\Psi_{\leq \omega_{+}}\right) n_{S_{\tau}}^{\mu} \leq \frac{C}{\tau-\frac{1}{4} t^{*}} \cdot\left\{e^{C \omega_{+}} \int_{t=0} J_{\mu}^{N}(\psi) n^{\mu}+\int_{t=0} r J_{\mu}^{N}(\psi) n^{\mu}\right\} .
$$


Interpolating (using Lemma 12.3 of the Appendix) between (8.10) and the boundedness estimate (following from Lemmas 4.4 and 4.6)

$$
\int_{S_{\tau} \cap \mathcal{R}\left(0, t^{*}\right)} J_{\mu}^{N}\left(\psi_{\leq \omega_{+}}\right) n_{S_{\tau}}^{\mu} \leq C \int_{t=0} J_{\mu}^{N}(\psi) n^{\mu}
$$

we readily obtain the desired estimate:

$$
\int_{S_{\tau} \cap \mathcal{R}\left(0, t^{*}\right)} J_{\mu}^{N}\left(\psi_{\leq \omega_{+}}\right) n_{S_{\tau}}^{\mu} \leq \frac{C}{\left(\tau-\frac{1}{4} t^{*}\right)^{\delta_{0}}} \cdot\left\{e^{C \omega_{+}} \int_{t=0} J_{\mu}^{N}(\psi) n^{\mu}+\int_{t=0} r^{\delta_{0}} J_{\mu}^{N}(\psi) n^{\mu}\right\} .
$$

\section{The Frequency Interpolation Argument}

In this section, we will fix $t^{*}, \omega_{0}$ and $\omega_{+}$, and we will complete the proof of Theorem 2.1 .

Let $\psi: \mathcal{D} \rightarrow \mathbb{C}$ be a smooth function as in the statement of Theorem 2.1, with compactly supported initial data on $\Sigma \cap \mathcal{D}$. For any given $\tau>0$, let $t^{*}=2 \tau$. Of course, we can safely asume without loss of generality that $\tau \gg 1$. Let us introduce a parameter $\omega_{+}>1$, and define $\psi_{t^{*}}, \psi_{\leq \omega_{+}}$and $\psi_{\geq \omega_{+}}$as in Section 4 .

Due to the fact that $\psi_{t^{*}}=\psi_{\leq \omega_{+}}+\psi_{\geq \omega_{+}}$, we can bound:

$$
\begin{aligned}
\int_{\{t=\tau\} \cap\left\{r \leq R_{1}\right\}} J_{\mu}^{N}(\psi) & =\int_{\{t=\tau\} \cap\left\{r \leq R_{1}\right\}} J_{\mu}^{N}\left(\psi_{t^{*}}\right) \lesssim \\
& \lesssim \int_{\{t=\tau\} \cap\left\{r \leq R_{1}\right\}} J_{\mu}^{N}\left(\psi_{\leq \omega_{+}}\right)+\int_{\{t=\tau\} \cap\left\{r \leq R_{1}\right\}} J_{\mu}^{N}\left(\psi_{\geq \omega_{+}}\right) .
\end{aligned}
$$

In view of Lemma 4.9, we can estimate for the high frequency part:

$$
\int_{\{t=\tau\} \cap\left\{r \leq R_{1}\right\}} J_{\mu}^{N}\left(\psi_{\geq \omega_{+}}\right) n^{\mu} \leq \frac{C_{m}}{\omega_{+}^{2 m}} \sum_{j=0}^{m} \int_{t=0} J_{\mu}^{N}\left(T^{j} \psi\right) n^{\mu} .
$$

In view of Proposition 8.1, we can estimate for the low frequency part:

$$
\int_{\{t=\tau\} \cap\left\{r \leq R_{1}\right\}} J_{\mu}^{N}\left(\psi_{\leq \omega_{+}}\right) \leq C \frac{1}{\tau^{\delta 0}} \cdot\left\{e^{C \omega_{+}} \int_{t=0} J_{\mu}^{N}(\psi) n^{\mu}+\int_{t=0} r^{\delta 0} J_{\mu}^{N}(\psi) n^{\mu}\right\} .
$$

From (9.1), (9.3) and (9.2), we thus deduce:

$$
\begin{aligned}
\int_{\{t=\tau\} \cap\left\{r \leq R_{1}\right\}} J_{\mu}^{N}(\psi) n^{\mu} \leq & C \frac{1}{\tau^{\delta 0}} \cdot\left\{e^{C \omega_{+}} \int_{t=0} J_{\mu}^{N}(\psi) n^{\mu}+\int_{t=0} r^{\delta 0} J_{\mu}^{N}(\psi) n^{\mu}\right\}+ \\
& +\frac{C_{m}}{\omega_{+}^{2 m}} \sum_{j=0}^{m} \int_{t=0} J_{\mu}^{N}\left(T^{j} \psi\right) n^{\mu}
\end{aligned}
$$


It is at this point that we will choose a suitable value for $\omega_{+}$. Choosing $\omega_{+}=$ $\frac{\log (2+\tau)}{2 C}$ in (9.4), we conclude:

$$
\begin{aligned}
\int_{\{t=\tau\} \cap\left\{r \leq R_{1}\right\}} J_{\mu}^{N}(\psi) n^{\mu} \leq & \frac{C_{m}}{\{\log (2+\tau)\}^{2 m}}\left(\sum_{j=0}^{m} \int_{t=0} J_{\mu}^{N}\left(T^{j} \psi\right) n^{\mu}\right) \\
& +\frac{C}{\tau \delta 0} \int_{t=0}(1+r)^{\delta 0} J_{\mu}^{N}(\psi) n^{\mu}
\end{aligned}
$$

and hence Theorem 2.12 has been established.

\section{Proof of Corollary 2.2}

In view of the boundedness assumption 4 and the conservation of the $J^{T}$ flux, it suffices to prove Corollary 2.2 for $S_{\tau}$ being the level sets $\{\bar{t}=\tau\}$ of the function $\bar{t}$ defined in Section 8.

Fix an $R_{1} \gg 1$ in the statement of Theorem 2.1. According to Theorem 2.12, for any $m \in \mathbb{N}$ and any $l>0$ (that will be fixed later), there exists a constant $C_{m}(l)>0$ (depending also on $R_{1}$ ) such that:

$$
\begin{aligned}
\int_{\{t=\tau\} \cap\left\{r \leq l \cdot R_{1}\right\}} J_{\mu}^{N}(\psi) n^{\mu} \leq & \frac{C_{m}(l)}{\{\log (2+\tau)\}^{2 m}}\left(\sum_{j=0}^{m} \int_{t=0} J_{\mu}^{N}\left(T^{j} \psi\right) n^{\mu}\right) \\
& +\frac{C_{m}(l)}{\tau^{\delta} 0} \int_{\{t=0\}}(1+r)^{\delta_{0}} \cdot J_{\mu}^{N}(\psi) n^{\mu} .
\end{aligned}
$$

Applying (5.33), we obtain for any $0 \leq t^{\prime} \leq t^{\prime \prime}: 34$

$$
\begin{aligned}
& \int_{t^{\prime}}^{t^{\prime \prime}}\left\{\int_{S_{\mathrm{s}} \cap\left\{r \geq R_{1}\right\}} J_{\mu}^{N}(\psi) n_{S}^{\mu}\right\} d s \leq C \cdot \int_{\mathcal{R}\left(t^{\prime}, t^{\prime \prime}\right) \cap\left\{R_{1} \leq r \leq R_{1}+1\right\}}\left(J_{\mu}^{N}(\psi) n^{\mu}+|\psi|^{2}\right) \\
& \quad+C \int_{\{t=0\}}(1+r)^{\delta_{0}} J_{\mu}^{N}(\psi) n^{\mu} .
\end{aligned}
$$

Using a Hardy inequality on $S_{\tau}$ (a corollary of (3.16)), we can bound:

$$
\begin{aligned}
\int_{S_{t} \cap\left\{R_{1} \leq r \leq R_{1}+1\right\}}|\psi|^{2} & \leq C \cdot \int_{S_{t} \cap\left\{R_{1} \leq r\right\}} r^{-2-\frac{1}{2}}|\psi|^{2} \\
& \leq C \cdot \int_{S_{t} \cap\left\{R_{1} \leq r\right\}} r^{-\frac{1}{2}} J_{\mu}^{N}(\psi) n_{S}^{\mu} .
\end{aligned}
$$

Thus, for any $\varepsilon>0$, if $l=l(\varepsilon)$ is chosen sufficiently large (independently of $t$ ), there exists a $C=C(\varepsilon)>0$ such that

\footnotetext{
34 Recall that $S_{t}$ and $\tilde{S}_{t}$ coincide for $\left\{r \geq R_{1}\right\}$ if $R_{1} \gg 1$.
} 


$$
\int_{S_{t} \cap\left\{R_{1} \leq r \leq R_{1}+1\right\}}|\psi|^{2} \leq \varepsilon \cdot \int_{S_{\mathrm{t}} \cap\left\{r \geq R_{1}\right\}} J_{\mu}^{N}(\psi) n_{S}^{\mu}+C(\varepsilon) \int_{S_{t} \cap\left\{r \leq l R_{1}\right\}} J_{\mu}^{N}(\psi) n_{S}^{\mu} .
$$

Returning to (10.2), in view of (10.4) we have for any $\varepsilon>0$ :

$$
\begin{aligned}
& \int_{t^{\prime}}^{t^{\prime \prime}}\left\{\int_{S_{\mathrm{s}} \cap\left\{r \geq R_{1}\right\}} J_{\mu}^{N}(\psi) n_{S}^{\mu}\right\} d s \leq C \varepsilon \cdot \int_{t^{\prime}}^{t^{\prime \prime}}\left\{\int_{S_{\mathrm{s}} \cap\left\{r \geq R_{1}\right\}} J_{\mu}^{N}(\psi) n_{S}^{\mu}\right\} d s \\
& +C(\varepsilon) \int_{t^{\prime}}^{t^{\prime \prime}}\left\{\int_{S_{t} \cap\left\{r \leq l \cdot R_{1}\right\}} J_{\mu}^{N}(\psi) n_{S}^{\mu}\right\} d s+C \int_{\{t=0\}}(1+r)^{\delta 0} J_{\mu}^{N}(\psi) n^{\mu},
\end{aligned}
$$

or, after absorbing the first term of the right hand side into the left hand side:

$$
\begin{aligned}
& \int_{t^{\prime}}^{t^{\prime \prime}}\left\{\int_{S_{S} \cap\left\{r \geq R_{1}\right\}} J_{\mu}^{N}(\psi) n_{S}^{\mu}\right\} d s \leq C(\varepsilon) \cdot \int_{t^{\prime}}^{t^{\prime \prime}}\left\{\int_{S_{t} \cap\left\{r \leq l \cdot R_{1}\right\}} J_{\mu}^{N}(\psi) n_{S}^{\mu}\right\} d s \\
& +C \int_{\{t=0\}}(1+r)^{\delta_{0}} J_{\mu}^{N}(\psi) n^{\mu} .
\end{aligned}
$$

From now on, $\varepsilon$ and hence $l$ will be considered fixed.

To make the notations a little simpler, let us for a moment denote

$$
f(t) \doteq \int_{S_{\mathrm{t}} \cap\left\{r \geq R_{1}\right\}} J_{\mu}^{N}(\psi) n_{S}^{\mu}
$$

for $t \geq 0$. We will also need the initial data quantities

$$
A \doteq \sum_{j=0}^{m} \int_{t=0} J_{\mu}^{N}\left(T^{j} \psi\right) n^{\mu}
$$

and

$$
B \doteq \int_{\{t=0\}}(1+r)^{\delta_{0}} J_{\mu}^{N}(\psi) n^{\mu}
$$

In view of (10.1), inequality (10.6) becomes (assuming without loss of generality that $\left.t^{\prime} \geq 1\right)$

$$
\begin{aligned}
\int_{t^{\prime}}^{t^{\prime \prime}} f(s) d s & \leq C_{m} \cdot A \cdot \int_{t^{\prime}}^{t^{\prime \prime}} \frac{1}{\{\log (s+2)\}^{2 m}} d s+C_{m} \cdot B \int_{t^{\prime}}^{t^{\prime \prime}} \frac{1}{s} d s \\
& \leq C_{m} \cdot(A+B) \cdot \int_{t^{\prime}}^{t^{\prime \prime}} \frac{1}{\{\log (s+2)\}^{2 m}} d s .
\end{aligned}
$$

Inequality (10.9), combined with an application of the pigeonhole principle, implies that there exists an infinite dyadic sequence $0<t_{1}<\ldots<t_{k}<\ldots$ of positive numbers with $t_{k+1} \in\left[2 t_{k}, 4 t_{k}\right],{ }^{35}$ such that $\forall j \in \mathbb{N}$ :

35 Thus $t_{j} \longrightarrow+\infty$ as $j \rightarrow+\infty$. 


$$
f\left(t_{j}\right) \leq 2 C_{m} \cdot(A+B) \cdot \frac{1}{\left\{\log \left(t_{j}+2\right)\right\}^{2 m}} .
$$

It is important that in this case, $C_{m}$ is the same constant as the one appearing in (10.9).

The existence of such a dyadic sequence follows by contradiction: If such a sequence did not exist, that would mean that for any $t_{1}>0$, there should exist a $t_{c}=t_{c}\left(t_{1}\right) \geq t_{1}$, such that for all $t \in\left[2 t_{c}, 4 t_{c}\right]$ :

$$
f(t)>2 C_{m} \cdot(A+B) \cdot \frac{1}{\{\log (t+2)\}^{2 m}} .
$$

But this would lead to a contradiction, since in this case due to (10.9) and (10.11) we could bound:

$$
\begin{aligned}
1 & \geq \limsup _{t_{1} \rightarrow+\infty} \frac{\int_{2 t_{c}}^{4 t_{c}} f(s) d s}{C_{m} \cdot(A+B) \cdot \int_{2 t_{c}}^{4 t_{c}} \frac{1}{\{\log (s+2)\}^{2 m}} d s} \\
& >\limsup _{t_{1} \rightarrow+\infty} \frac{2 C_{m} \cdot(A+B) \cdot \int_{2 t_{c}}^{4 t_{c}} \frac{1}{\{\log (s+2)\}^{2 m}} d s}{C_{m} \cdot(A+B) \cdot \int_{2 t_{c}}^{4 t_{c}} \frac{1}{\{\log (s+2)\}^{2 m}} d s}=2 .
\end{aligned}
$$

We also note that due to the boundedness assumption $4,{ }^{36}$ there exists a constant $C>0$ (independent, of course, of $\psi$ ) such that for any $t$ in any interval $\left[t_{j}, t_{j+1}\right]$ defined by the previous dyadic sequence, we can bound

$$
f(t) \leq C \cdot f\left(t_{j}\right)
$$

Thus, since the sequence $\left\{t_{j}\right\}_{j \in \mathbb{N}}$ satisfied $t_{j+1} \in\left[2 t_{j}, 4 t_{j}\right]$, we conclude that for any $t \geq 0$ :

$$
f(t) \leq 2 C_{m} \cdot(A+B) \cdot \frac{1}{\{\log (t+2)\}^{2 m}},
$$

and thus Corollary 2.2 has been established.

\section{Proof of Corollaries 2.3 and 2.4}

In this Section, we will establish Corollaries 2.3 and 2.4 using Corollary 2.2.

\subsection{Proof of Corollary 2.3}

In order to show that $\lim _{\tau \rightarrow+\infty} \int_{S_{\tau}} J_{\mu}^{N}(\psi) n_{S}^{\mu}=0$, it suffices to show that for any given $\varepsilon>0$, there exists some $\tau_{0}>0$ such that for any $\tau \geq \tau_{0}: \int_{S_{\tau}} J_{\mu}^{N}(\psi) n_{S}^{\mu}<\varepsilon$.

\footnotetext{
36 Notice the remark following assumption 4.
} 
Since $\int_{t=0} J_{\mu}^{N}(\psi) n^{\mu}<\infty$, using the standard density arguments we can find smooth and compactly supported initial data $\left(\tilde{\psi}, \partial_{t} \tilde{\psi}\right)$ on $t=0$, with the property that $\int_{t=0} J_{\mu}^{N}(\psi-\tilde{\psi}) n^{\mu}<0$.

Let $\tilde{\psi}$ be the unique solution of $\square_{g} \tilde{\psi}=0$ on $\mathcal{D}$ with initial data $\left.\left(\tilde{\psi}, \partial_{t} \tilde{\psi}\right)\right|_{t=0}$. Then, due to the linearity of the wave operator, $\psi-\tilde{\psi}$ will also solve $\square_{g}(\psi-\tilde{\psi})=0$. Due to the boundedness assumption 4 and the conservation of the $J^{T}$-flux, we can then bound

$$
\int_{S_{\tau}} J_{\mu}^{N}(\psi-\tilde{\psi}) n_{S}^{\mu}<C \cdot \int_{t=0} J_{\mu}^{N}(\psi-\tilde{\psi}) n^{\mu}<<C \cdot \varepsilon .
$$

Moreover, since $\left.\left(\tilde{\psi}, \partial_{t} \tilde{\psi}\right)\right|_{t=0}$ is smooth and compactly supported and thus has finite $\int_{t=0} r \cdot J_{\mu}^{N}(\tilde{\psi}) n^{\mu}$ and $\int_{t=0} J_{\mu}^{N}(T \tilde{\psi}) n^{\mu}$ norms, Corollary 2.2 applies to show that

$$
\int_{S_{\tau}} J_{\mu}^{N}(\tilde{\psi}) n_{S}^{\mu} \leq \frac{C(\tilde{\psi})}{\{\log (2+\tau)\}^{2}}
$$

Thus, there exists some $\tau_{0}$ depending on $\tilde{\psi}$ itself such that for $\tau \geq \tau_{0}$ :

$$
\int_{S_{\tau}} J_{\mu}^{N}(\tilde{\psi}) n_{S}^{\mu}<\varepsilon
$$

Combining (11.1) and (11.3), and using a triangle inequality, we thus obtain the required estimate:

$$
\int_{S_{\tau}} J_{\mu}^{N}(\psi) n_{S}^{\mu}<C \cdot \varepsilon
$$

The claim that $\lim _{\tau \rightarrow+\infty} \int_{S_{\tau}} J_{\mu}^{T}(\psi) n_{S}^{\mu}=0$ if $\int_{t=0} J_{\mu}^{T}(\psi) n^{\mu}<\infty$ in the case the $J^{T}$-energy is coercive follows in exactly the same way, using the conservation of the $J^{T}$-current in place of the boundedness of the $J^{N}$-energy.

\subsection{Proof of Corollary 2.4}

Assume that $(\mathcal{D}, g)$ satisfies the assumptions of Corollary 2.4. We will also assume without loss of generality that $\mathcal{I}_{a s}$ has only one connected component (see also Section 2.6).

We will first show the first part of Corollary 2.4, that is to say, the statement that for any solution $\psi$ to the wave equation $\square_{g} \psi=0$ on $\mathcal{D}$ with

$$
\int_{t=0} J_{\mu}^{T}(\psi) n^{\mu}<\infty
$$

the following equality holds:

$$
\int_{\mathcal{H}^{+} \cap\{t \geq 0\}} J_{\mu}^{T}(\psi) n_{\mathcal{H}^{+}}^{\mu}+\int_{\mathcal{I}^{+}} J_{\mu}^{T}(\psi) n_{\mathcal{I}^{+}}^{\mu}=\int_{t=0} J_{\mu}^{T}(\psi) n^{\mu} .
$$


Let $\left\{S_{\tau}\right\}_{\tau \in \mathbb{R}}$ be the level sets of the function $\bar{t}$ constructed in Section 8. We can assume without loss of generality (after setting $\bar{t} \rightarrow \bar{t}+c)$ that $S_{0} \subset J^{+}(\{t=0\})$. Moreover, in view of the definition of the future radiation field on $\mathcal{I}^{+}$for solutions to (1.1) with merely finite initial energy norm (see the remarks above the statement of Corollary 2.25), we will assume without loss of generality that $\psi$ is smooth and induces compactly supported initial data on $\Sigma \cap \mathcal{D}$.

The conservation of the $J_{\mu}^{T}(\psi)$ current in the region bounded by $\{t=0\}, S_{\tau}, \mathcal{H}^{+}$ and a null hyperurface $\mathcal{I}_{n, \tau}^{+}$of $\mathcal{M}$ of the form

$$
\mathcal{I}_{n, \tau}^{+} \doteq \partial J^{-}\left(S_{\tau} \cap\left\{r \leq R_{n}\right\}\right) \backslash \mathcal{H}^{+}
$$

for a sequence $R_{n} \rightarrow+\infty$ yields:

$$
\begin{aligned}
\int_{\{t=0\} \cap J^{-}\left(S_{\tau} \cap\left\{r \leq R_{n}\right\}\right)} J_{\mu}^{T}(\psi)= & \int_{\mathcal{H}^{+} \cap J^{-}\left(S_{\tau}\right) \cap\{t \geq 0\}} J_{\mu}^{T}(\psi) n_{\mathcal{H}^{+}}^{\mu}+\int_{S_{\tau} \cap\left\{r \leq R_{n}\right\}} J_{\mu}^{T}(\psi) n_{S}^{\mu}+ \\
& +\int_{\mathcal{I}_{n, \tau}^{+} \cap J^{-}\left(S_{\tau}\right)} J_{\mu}^{T}(\psi) n_{\mathcal{I}_{n, \tau}^{+}}^{\mu}
\end{aligned}
$$

The identity (11.8) implies that

$$
\sup _{n} \int_{\mathcal{I}_{n, \tau}^{+} \cap J^{-}\left(S_{\tau}\right)} J_{\mu}^{T}(\psi) n_{\mathcal{I}_{n, \tau}^{+}}^{\mu} \leq \int_{t=0} J_{\mu}^{T}(\psi) n^{\mu}<+\infty .
$$

Moreover, since $\psi$ was assumed to induce compactly supported initial data on $\{t=0\}$, there exist $R_{0} \gg 1$ and $u_{0} \in \mathbb{R}$ such that (in view of the domain of dependence property for the wave equation)

$$
\operatorname{supp}(\psi) \cap\left\{r \geq R_{0}\right\} \subset\left\{u \geq u_{0}\right\}
$$

(where the function $u$ is constructed in the Appendix). Therefore, in view of (11.9), (11.10) and the fact that $r^{\frac{d-1}{2}} \psi(u, r, \sigma) \rightarrow \Psi_{\mathcal{I}^{+}}(u, \sigma)$ in the $H_{l o c}^{1}\left(\mathbb{R} \times \mathbb{S}^{d-1}, d u^{2}+\right.$ $g_{\mathbb{S}^{d-1}}$ ) norm (see the remarks above the statement of Corollary 2.4), the dominated convergence theorem implies that

$$
\int_{\mathcal{I}_{n}^{+}} J_{\mu}^{T}(\psi) n_{\mathcal{I}_{n}^{+}}^{\mu} \rightarrow \int_{\mathcal{I}^{+} \cap J^{-}\left(S_{\tau}\right)} J_{\mu}^{T}(\psi) n_{\mathcal{I}^{+}}^{\mu},
$$

and thus (11.8) readily implies for any $\tau \geq 0$ (in the limit $n \rightarrow+\infty$ ):

$$
\begin{aligned}
\int_{t=0} J_{\mu}^{T}(\psi)= & \int_{\mathcal{H}^{+} \cap J^{-}\left(S_{\tau}\right) \cap\{t \geq 0\}} J_{\mu}^{T}(\psi) n_{\mathcal{H}^{+}}^{\mu}+\int_{S_{\tau}} J_{\mu}^{T}(\psi) n_{S}^{\mu}+ \\
& +\int_{\mathcal{I}^{+} \cap J^{-}\left(S_{\tau}\right)} J_{\mu}^{T}(\psi) n_{\mathcal{I}^{+}}^{\mu},
\end{aligned}
$$

where $\int_{\mathcal{I}^{+} \cap J^{-}\left(S_{\tau}\right)} J_{\mu}^{T}(\psi) n_{\mathcal{I}^{+}}^{\mu}$ is defined as (2.21). 
According to Corollary 2.3, since $\int_{t=0} J_{\mu}^{T}(\psi) n^{\mu}<\infty$ we also have:

$$
\lim _{\tau \rightarrow \infty} \int_{S_{\tau}} J_{\mu}^{T}(\psi) n_{S}^{\mu}=0
$$

Thus, in the limit $\tau \rightarrow+\infty$, the relation (11.12) yields the desired identity (11.6).

In order to complete the proof of Corollary 2.4, it remains to show that for any pair $\left(\left.\psi\right|_{\mathcal{H}^{+}}, \Psi_{\mathcal{I}^{+}}\right)$with finite $J^{T}$-energy, there exists an $H_{l o c}^{1}$ solution $\psi$ to the wave equation $\square_{g} \psi=0$ on $\mathcal{D}$ with $\int_{t=0} J_{\mu}^{T}(\psi) n^{\mu}<\infty$, which gives rise to the scattering data pair $\left(\left.\psi\right|_{\mathcal{H}^{+}}, \Psi_{\mathcal{I}^{+}}\right)$. To this end, let us define Hilbert space $\mathscr{E}_{0}$ of finite energy initial data on $\{t=0\}$ consisting of the completion of the space $C_{0}^{\infty}(\{t=0\}) \times C_{0}^{\infty}(\{t=0\})$ with the $J^{T}$-norm:

$$
\left\|\left(\psi_{0}, \psi_{1}\right)\right\|_{J^{T}} \doteq \int_{t=0} J_{\mu}^{T}(\psi) n^{\mu},
$$

where $\psi$ is the unique function defined on $\{t \geq 0\} \cap \mathcal{D}$ such that $\left.\psi\right|_{t=0}=\psi_{0}$ and $\left.T \psi\right|_{t=0}=\psi_{1}$. Let us also define the Hilbert space $\mathscr{E}_{S c}$ of finite energy scattering data as the completion of the space $C_{0}^{\infty}\left(\mathcal{H}^{+} \cap\{t \geq 0\}\right) \times C_{0}^{\infty}\left(\mathcal{I}^{+}\right)\left(\right.$where $\left.\mathcal{I}^{+} \simeq \mathbb{R} \times \mathbb{S}^{d-1}\right)$ with the norm

$$
\left\|\left(\left.\psi\right|_{\mathcal{H}^{+} \cap\{t \geq 0\}}, \Psi_{\mathcal{I}^{+}}\right)\right\|_{\mathscr{E}_{s c}} \doteq \int_{\mathcal{H}^{+} \cap\{t \geq 0\}} J_{\mu}^{T}(\psi) n_{\mathcal{H}^{+}}^{\mu}+\int_{\mathcal{I}^{+}} J_{\mu}^{T}(\psi) n_{\mathcal{I}^{+}}^{\mu} .
$$

We will also define the scattering map Scatt $: \mathscr{E}_{0} \rightarrow \mathscr{E}_{S c}$, which maps any pair of initial data $\left(\psi_{0}, \psi_{1}\right)$ to the scattering data $\left(\left.\psi\right|_{\mathcal{H}^{+} \cap\{t \geq 0\}}, \Psi_{\mathcal{I}^{+}}\right)$, induced by the solution $\psi$ to $\square_{g} \psi=0$ with initial data $\left.\psi\right|_{t=0}=\psi_{0}$ and $\left.T \psi\right|_{t=0}=\psi_{1}$, according to the remark above the statement of Corollary 2.4.

With these notations, in order to complete the proof of Corollary 2.4, we have to show that the scattering map Scatt is onto. In view of (11.6), Scatt is an isometric embedding. Thus, it suffices to show that its image is dense in $\mathcal{E}_{s c}$. In particular, we will show that for any scattering data pair $\left(\left.\psi\right|_{\mathcal{H}^{+} \cap\{t \geq 0\}}, \Psi_{\mathcal{I}^{+}}\right)$which are smooth and compactly supported and any $\varepsilon>0$, there exists a solution $\tilde{\psi}$ to the wave equation $\square \tilde{\psi}=0$ on $\{t \geq 0\} \cap \mathcal{D}$ with $\int_{t=0} J_{\mu}^{T}(\tilde{\psi}) n^{\mu}<+\infty$, such that

$$
\|\left(\left.\tilde{\Psi}\right|_{\mathcal{H}^{+} \cap\{t \geq 0\}}, \tilde{\Psi}_{\mathcal{I}^{+}}\right)-\left.\left(\left.\psi\right|_{\mathcal{H}^{+} \cap\{t \geq 0\}}, \Psi_{\mathcal{I}^{+}}\right)\right|_{\mathscr{E}_{s c}}<\varepsilon
$$

where $\tilde{\Psi}_{\mathcal{I}}$ is the future radiation field of $\tilde{\psi}$.

We will assume without loss of generality that the scattering data pair $\left(\left.\psi\right|_{\mathcal{H}^{+} \cap\{t \geq 0\}}\right.$, $\left.\Psi_{\mathcal{I}^{+}}\right)$is real valued. Since $\left(\left.\psi\right|_{\mathcal{H}^{+} \cap\{t \geq 0\}}, \Psi_{\mathcal{I}^{+}}\right)$was assumed to be compactly supported, there exists some $t_{f}>0$ such that $\left.\psi\right|_{\mathcal{H}^{+} \cap\left\{t \geq t_{f}\right\}} \equiv 0$ and $\left.\Psi_{\mathcal{I}^{+}}\right|_{\left\{u \geq t_{f}\right\}} \equiv 0$. Moreover, there exists some $t_{\text {in }}<0$ such that $\left.\Psi_{\mathcal{I}^{+}}\right|_{\left\{u \leq t_{\text {in }}\right\}} \equiv 0$.

Let us also introduce the $C^{1}$ optical function $\bar{v}:\left\{r \geq R_{f a r}\right\} \cap\left\{\bar{t} \leq t_{f}\right\} \rightarrow \mathbb{R}$ such that the level sets of $\bar{v}$ are the hypersurfaces

$$
\mathcal{I}_{R}^{+} \doteq \partial\left(J^{-}\left(S_{t_{f}} \cap\{r \leq R\}\right) \backslash \mathcal{H}^{+}\right.
$$

and satifying $T \bar{v}=1$. 
In the region $\left\{\bar{t} \leq t_{f}\right\} \cap\left\{\bar{v} \geq v_{0}\right\} \subset \mathcal{D}$ for some $v_{0} \gg 1$, we will introduce the flat metric associated to the $(u, v, \sigma)$ coordinate chart there:

$$
g_{f l}=-4 d u d v+r^{2} g_{\mathbb{S}^{d-1}}
$$

In this region, we can construct a solution $\psi_{f l}$ to the flat wave equation by solving the following scattering problem:

$$
\begin{cases}\square_{g_{f l}} \psi_{f l}=0 & \text { on }\left\{\bar{t} \leq t_{f}\right\} \cap\left\{\bar{v} \geq v_{0}\right\} \\ \left.\left(\psi_{f l}, T \psi_{f l}\right)\right|_{S_{f} \cap\left\{\bar{v} \geq v_{0}\right\}}=0 & \\ \lim _{v \rightarrow+\infty} r^{\frac{d-1}{2}} \psi_{f l}(u, v, \sigma)=\Psi_{\mathcal{I}^{+}}(u, \sigma) . & \end{cases}
$$

Notice that the scattering problem (11.19) is a scattering problem on Minkowski spacetime, and the existence and uniqueness of a solution $\psi_{f l}$ to (11.19) follows readily by using the confromal compactification method and solving (backwards) a regular mixed characteristic-initial value problem for the conformal wave equation (in the setup of [11]). Alternatively, one can use the $r^{p}$-weighted energy method of [20], see Section 9.6 of [24]. Following either way of proof, one obtains a unique smooth solution $\psi_{f l}$ to (11.19) satisfying the following qualititative bound

$$
\sum_{j=0}^{k} \sum_{j_{1}+j_{2}+j_{3}=j} \int_{\{t \geq 0\} \cap\left\{\bar{t} \leq t_{f}\right\} \cap\left\{\bar{v} \geq v_{0}\right\}} r^{-1-\delta} \cdot\left|r^{j_{1}} \partial_{v}^{j_{1}} \partial_{\sigma}^{j_{2}} \partial_{u}^{j_{3}}\left(r^{\frac{d-1}{2}} \Psi_{f l}\right)\right|^{2} d u d v d \sigma<+\infty
$$

for any $k \in \mathbb{N}$ and any $\delta>0$.

In view of (11.20), for any $C_{0}>0$ (to be fixed later), there exists a $v_{\varepsilon} \gg v_{0}$ (depending on $\varepsilon, \delta, C_{0}$ and the function $\psi_{f l}$ itself) such that

$$
\sum_{j=0}^{3} \sum_{j_{1}+j_{2}+j_{3}=j} \int_{\{t \geq 0\} \cap\left\{\bar{t} \leq t_{f}\right\} \cap\left\{\bar{v} \geq v_{\varepsilon}\right\}} r^{-1-\delta} \cdot\left|r^{j_{1}} \partial_{v}^{j_{1}} \partial_{\sigma}^{j_{2}} \partial_{u}^{j_{3}}\left(r^{\frac{d-1}{2}} \psi_{f l}\right)\right|^{2} d u d v d \sigma<C_{0}^{-1} \varepsilon
$$

Let $R_{\varepsilon}>1$ be fixed so that $\left\{\bar{t}=t_{f}\right\} \cap\left\{\bar{v}=v_{\varepsilon}\right\} \subset\left\{R_{\varepsilon}-1 \leq r \leq R_{\varepsilon}\right\}$. Then, there exists a smooth spacelike hypersurface $\bar{S}_{\varepsilon}$ of $(\mathcal{D}, g)$ (depending again on $\varepsilon, \delta, C_{0}$ and $\left.\psi_{f l}\right)$ such that $\bar{S}_{\varepsilon} \equiv\left\{t=R_{\varepsilon}+2 v_{\varepsilon}\right\}$ in the region $\left\{r \geq R_{\varepsilon}+1\right\}$ and $\bar{S}_{\varepsilon} \equiv S_{t_{f}}$ in the region $\left\{r \leq R_{\varepsilon}\right\}$. Notice that the domain of dependence of $\bar{S}_{\varepsilon} \cup\left(\mathcal{H}^{+} \cap\{t \geq 0\}\right)$ contains the whole of $\{t \geq 0\}$.

We will construct the solution $\tilde{\psi}$ to $\square_{g} \tilde{\psi}=0$ on $\{t \geq 0\}$ with initial-characteristic data on $\bar{S}_{\varepsilon} \cup\left(\mathcal{H}^{+} \cap\{t \geq 0\}\right.$ as follows:

$$
\begin{cases}\square_{g} \tilde{\psi}=0 & \text { on } J^{+}(\Sigma) \cap \mathcal{D} \\ \left.(\tilde{\psi}, T \tilde{\psi})\right|_{\bar{S}_{\varepsilon} \cap\left\{r \leq R_{\varepsilon}-1\right\}}=(0,0) & \\ \left.(\tilde{\psi}, T \tilde{\psi})\right|_{\bar{S}_{\varepsilon} \cap\left\{r \geq R_{\varepsilon}-1\right\}}=\left.\left(\psi_{f l}, T \psi_{f l}\right)\right|_{\bar{S}_{\varepsilon} \cap\left\{r \geq R_{\varepsilon}-1\right\}} & \\ \left.\tilde{\psi}\right|_{\mathcal{H}^{+} \cap\{t \geq 0\}}=\left.\psi\right|_{\mathcal{H}^{+} \cap\{t \geq 0\}} . & \end{cases}
$$


Notice that the initial data for $\tilde{\psi}$ on $\bar{S}_{\varepsilon}$ are smooth, in view of the fact that $\left.\left(\psi_{f l}, T \psi_{f l}\right)\right|_{S_{f} \cap\left\{\bar{v} \geq v_{0}\right\}}=0$. Hence, $\tilde{\psi}$ is a smooth function on $\{t \geq 0\}$.

We will now show that the radiation field of $\tilde{\psi}$ on $\mathcal{I}^{+}$exists in the $H_{l o c}^{1}$ norm and is actually $\varepsilon$-close to $\Psi_{\mathcal{I}^{+}}$in the $\int_{\mathcal{I}^{+}} J_{\mu}^{T}(\cdot) n_{\mathcal{I}^{+}}^{\mu}$ norm. Notice that for any smooth function $\omega$ on $\left\{\bar{t} \leq t_{f}\right\} \cap\left\{\bar{v} \geq v_{0}\right\}$ we can estimate

$$
\begin{aligned}
r^{\frac{d-1}{2}} \square_{g} \omega= & r^{\frac{d-1}{2}} \square_{f l} \omega+O_{3}\left(r^{-2-a}\right) \partial_{u}^{2}\left(r^{\frac{d-1}{2}} \omega\right)+O_{3}\left(r^{-1}\right) \partial_{v}^{2}\left(r^{\frac{d-1}{2}} \omega\right) \\
& +O_{3}\left(r^{-2-a}\right) \partial_{u} \partial_{\sigma}\left(r^{\frac{d-1}{2}} \omega\right)+O_{3}\left(r^{-2}\right) \partial_{v} \partial_{\sigma}\left(r^{\frac{d-1}{2}} \omega\right) \\
& +O_{2}\left(r^{-3-a}\right) \partial_{\sigma} \partial_{\sigma}\left(r^{\frac{d-1}{2}} \omega\right)+O_{2}\left(r^{-2-a}\right) \partial_{u}\left(r^{\frac{d-1}{2}} \omega\right) \\
& +O_{2}\left(r^{-1-a}\right) \partial_{v}\left(r^{\frac{d-1}{2}} \omega\right)+O_{2}\left(r^{-2-a}\right) \partial_{\sigma}\left(r^{\frac{d-1}{2}} \omega\right)+O_{1}\left(r^{-3}\right)\left(r^{\frac{d-1}{2}} \omega\right) .
\end{aligned}
$$

Remark Notice that the precise decay rate in $r$ of the coefficients in (11.23) reflects the fact that the long range part of the metric $g$ (viewed as a perturbation of the flat metric $g_{f l}$ ) is spherically symmetric.

In view of (11.19), (11.22) and (11.23) for $\psi_{f l}$ in place of $\omega$, the difference $\tilde{\psi}-\psi_{f l}$ solves the following initial value problem on $\left\{\bar{t} \leq t_{f}\right\} \cap J^{+}\left(\bar{S}_{\varepsilon}\right)$ :

$$
\left\{\begin{array}{l}
\square_{g}\left(\tilde{\psi}-\psi_{f l}\right)=F_{f l} \\
\left.\left(\tilde{\psi}-\psi_{f l}, T\left(\tilde{\psi}-\psi_{f l}\right)\right)\right|_{\left\{\bar{t} \leq t_{f}\right\} \cap \bar{S}_{\varepsilon}}=0,
\end{array} \text { on }\left\{\bar{t} \leq t_{f}\right\} \cap J^{+}\left(\bar{S}_{\varepsilon}\right)\right.
$$

where, in view of (11.23) and (11.21) we can estimate (for some $\delta_{0}>0$ small in terms of $\delta, a$ )

$$
\sum_{j=0}^{1} \sum_{j_{1}+j_{2}+j_{3}=j} \int_{\left\{\bar{t} \leq t_{f}\right\} \cap J^{+}\left(\bar{S}_{\varepsilon}\right)} r^{1+\delta_{0}}\left|r^{j_{1}} \partial_{v}^{j_{1}} \partial_{\sigma}^{j_{2}} \partial_{u}^{j_{3}}\left(r^{\frac{d-1}{2}} F_{f l}\right)\right|^{2} d u d v d \sigma \leq C \cdot C_{0}^{-1} \varepsilon .
$$

Repeating the proof of Theorem 7.1 of [52] for $\tilde{\psi}-\psi_{f l}$ in the region $\left\{\bar{t} \leq t_{f}\right\} \cap$ $J^{+}\left(\bar{S}_{\varepsilon}\right),(11.24)$ and (11.25) imply that for any increasing sequence $\left\{v_{n}\right\}_{n \in \mathbb{N}}$ with $v_{n} \rightarrow+\infty$, the sequence $r^{\frac{d-1}{2}}\left(\tilde{\psi}-\psi_{f l}\right)\left(u, v_{n}, \sigma\right)$ converges in the $H_{l o c}^{1}$ norm. Since by construction of the function $\psi_{f l}$ the sequence $r^{\frac{d-1}{2}} \psi_{f l}\left(u, v_{n}, \sigma\right)$ converges in the $H_{l o c}^{1}$ topology, we also deduce that the sequence $r^{\frac{d-1}{2}} \tilde{\psi}\left(u, v_{n}, \sigma\right)$ converges in the $H_{l o c}^{1}$ norm, and we set

$$
\tilde{\Psi}_{\mathcal{I}^{+}}(u, \sigma) \doteq \lim _{v \rightarrow+\infty}\left(r^{\frac{d-1}{2}} \tilde{\psi}(u, v, \sigma)\right)
$$

Furthermore, by applying the energy identity for the $T$ vector field on the region $\left\{\bar{t} \leq t_{f}\right\} \cap J^{+}\left(\bar{S}_{\varepsilon}\right)$, we infer from (11.24) and (11.25) that:

$$
\int_{\mathcal{I}^{+} \cap\left\{\bar{t} \leq t_{f}\right\}} J_{\mu}^{T}\left(\tilde{\Psi}-\psi_{f l}\right) n_{\mathcal{I}^{+}}^{\mu} \leq C \cdot C_{0}^{-1} \varepsilon
$$


Since the function $\psi_{f l}$ solves

$$
\square_{g} \psi_{f l}=-F_{f l}
$$

and satisfies (11.19), by applying the energy identity for the $T$ vector field on the region $\left\{\bar{t} \leq t_{f}\right\} \cap J^{+}\left(\bar{S}_{\varepsilon}\right)$ in the backward evolution (notice that $\bar{S}_{\varepsilon} \cap\left\{\bar{t} \leq t_{f}\right\}$ lies in the domain of dependence of $\left\{\bar{t}=t_{f}\right\} \cup\left(\mathcal{I}^{+} \cap\left\{u \leq t_{f}\right\}\right.$ ), we can bound (in view of $(11.25))$ :

$$
\int_{\bar{S}_{\varepsilon} \cap\left\{\bar{t} \leq t_{f}\right\}} J_{\mu}^{T}\left(\psi_{f l}\right) n^{\mu} \leq \int_{\mathcal{I}^{+}} J_{\mu}^{T}(\psi) n_{\mathcal{I}^{+}}^{\mu}+C \cdot C_{0}^{-1} \varepsilon .
$$

Thus, since

$$
\left.(\tilde{\psi}, T \tilde{\psi})\right|_{\bar{S}_{\varepsilon} \cap\left\{r \geq R_{\varepsilon}-1\right\}}=\left.\left(\psi_{f l}, T \psi_{f l}\right)\right|_{\bar{S}_{\varepsilon} \cap\left\{r \geq R_{\varepsilon}-1\right\}}
$$

and

$$
\left.(\tilde{\psi}, T \tilde{\psi})\right|_{\bar{S}_{\varepsilon} \cap\left\{r \leq R_{\varepsilon}-1\right\}}=(0,0),
$$

(11.29) immediately yields:

$$
\int_{\bar{S}_{\varepsilon}} J_{\mu}^{T}(\tilde{\psi}) n_{\bar{S}_{\varepsilon}}^{\mu} \leq \int_{\mathcal{I}^{+}} J_{\mu}^{T}(\psi) n^{\mu}+C \cdot C_{0}^{-1} \varepsilon,
$$

where $n_{\bar{S}_{\varepsilon}}$ is the future directed unit normal of the hypersurface $\bar{S}_{\varepsilon}$. Therefore, in view of the fact that $\left.\tilde{\psi}\right|_{\mathcal{H}^{+} \cap\{t \geq 0\}}=\left.\psi\right|_{\mathcal{H}^{+} \cap\{t \geq 0\}}$, by applying once more the energy identity for the $T$ vector field in the region boundd by $\{t=0\}, \mathcal{H}^{+}$and $\bar{S}_{\varepsilon}$, we obtain (using (11.32)):

$$
\int_{t=0} J_{\mu}^{T}(\tilde{\psi}) n^{\mu} \leq \int_{\mathcal{H}^{+} \cap\{t \geq 0\}} J_{\mu}^{T}(\psi) n_{\mathcal{H}^{+}}^{\mu}+\int_{\mathcal{I}^{+}} J_{\mu}^{T}(\psi) n_{\mathcal{I}^{+}}^{\mu}+C \cdot C_{0}^{-1} \varepsilon .
$$

Since $\int_{t=0} J_{\mu}^{T}(\tilde{\psi}) n^{\mu}<+\infty$ (in view of (11.33)), according to the definition above the statement of Corollary 2.4 the radiation field $\tilde{\Psi}_{\mathcal{I}^{+}}$of $\tilde{\psi}$ on $\mathcal{I}^{+}$exists in the Hilbert space defined by the $\int_{\mathcal{I}^{+}} J_{\mu}^{T}(\cdot) n_{\mathcal{I}^{+}}^{\mu}$ norm, and its restriction on $\mathcal{I}^{+} \cap\left\{u \leq t_{f}\right\}$ coincides with the limit (11.26). In view of (2.23), which also applies for $\tilde{\psi}$, we can bound:

$$
\int_{\mathcal{H}^{+} \cap\{t \geq 0\}} J_{\mu}^{T}(\tilde{\psi}) n_{\mathcal{H}^{+}}^{\mu}+\int_{\mathcal{I}^{+}} J_{\mu}^{T}(\tilde{\psi}) n_{\mathcal{I}^{+}}^{\mu} \leq \int_{t=0} J_{\mu}^{T}(\tilde{\psi}) n^{\mu} .
$$

Thus, from (11.33) and (11.34) (using also the fact that $\left.\tilde{\psi}\right|_{\mathcal{H}^{+} \cap\{t \geq 0\}}=\left.\psi\right|_{\mathcal{H}^{+} \cap\{t \geq 0\}}$ ) we infer:

$$
\int_{\mathcal{I}^{+}} J_{\mu}^{T}(\tilde{\psi}) n_{\mathcal{I}^{+}}^{\mu} \leq \int_{\mathcal{I}^{+}} J_{\mu}^{T}(\psi) n_{\mathcal{I}^{+}}^{\mu}+C \cdot C_{0}^{-1} \varepsilon .
$$

From (11.27) and the fact that $\Psi_{\mathcal{I}^{+}}$was assumed to be supported on $\left\{u \leq t_{f}\right\}$, we obtain:

$$
\int_{\mathcal{I}^{+} \cap\left\{u \leq t_{f}\right\}} J_{\mu}^{T}(\tilde{\psi}) n_{\mathcal{I}^{+}}^{\mu} \geq \int_{\mathcal{I}^{+}} J_{\mu}^{T}(\psi) n_{\mathcal{I}^{+}}^{\mu}-C \cdot C_{0}^{-1} \varepsilon .
$$


Therefore, from (11.35) and (11.36) (as well as the fact that $\Psi_{\mathcal{I}^{+}} \equiv 0$ on $\left\{u \geq t_{f}\right\}$ we deduce:

$$
\int_{\mathcal{I}^{+} \cap\left\{u \geq t_{f}\right\}} J_{\mu}^{T}(\tilde{\psi}-\psi) n_{\mathcal{I}^{+}}^{\mu}=\int_{\mathcal{I}^{+} \cap\left\{u \geq t_{f}\right\}} J_{\mu}^{T}(\tilde{\psi}) n_{\mathcal{I}^{+}}^{\mu} \leq 2 C \cdot C_{0}^{-1} \varepsilon
$$

which, after adding (11.27) and assuming $C_{0}$ was chosen large enough in terms of the geometry of $(\mathcal{D}, g)$, yields:

$$
\int_{\mathcal{I}^{+}} J_{\mu}^{T}(\tilde{\psi}-\psi) n_{\mathcal{I}^{+}}^{\mu} \leq \varepsilon
$$

Combining (11.38) with the fact that $\left.\tilde{\psi}\right|_{\mathcal{H}^{+} \cap\{t \geq 0\}}=\left.\psi\right|_{\mathcal{H}^{+} \cap\{t \geq 0\}}$, we immediately infer the desired estimate (11.16). Thus, the proof of Corllary $2 . \overline{4}$ is complete.

\section{Sharpness of the Logarithmic Decay Statement}

In this section, we will construct a spacetime $(\mathcal{M}, g)$ satisfying all four assumptions 1 , 2,3 and 4, for which the logarithmic decay rate of Theorem 2.1 is optimal. This example is a modification of a counterexample provided by Rodnianski and Tao in [58], in the context of their proof of effective limiting absorption principles for asymptotically conic Riemannian manifolds, which in our setting correspond to ultrastatic spacetimes (namely static spacetimes with $g(T, T) \equiv-1$ ).

We should notice, however, that a more realistic class of spacetimes exhibiting the optimality of Theorem 2.1 has been investigated by Keir in the recent [44]. This class includes the family of spherically symmetric ultracompact neutron stars. The absence of a horizon and an ergoregion, as well as the consrvation of a positive definite energy makes these spacetimes valid backgrounds on which Theorem 2.1 holds.

The construction of a simple spacetime $(\mathcal{M}, g)$ exhibiting the optimality of Theorem 2.1 proceeds as follows: We consider $\mathbb{R}^{3}$ equipped with a smooth Riemannian metric $g_{0}$ with the following behaviour: In the usual polar coordinate system $(r, \vartheta, \omega)$, we assume that $g_{0}$ takes the folowing form:

$$
g_{0}=d r^{2}+f(r) \cdot\left(d \vartheta^{2}+\sin ^{2} \vartheta \cdot d \omega^{2}\right),
$$

where $f:[0,+\infty) \rightarrow[0,+\infty)$ is a smooth function such that

- $f(r)=\sin ^{2} r$ for $0 \leq r \leq \frac{7 \pi}{8}$,

- $f(r)=r^{2}$ for $r \geq \pi$ and

- $f(r)>0$ for $\frac{7 \pi}{8} \leq r \leq \pi$.

That is to say, $g_{0}$ coincides with the usual Euclidean metric for $r \geq \pi$, while the region $r \leq \frac{7 \pi}{8}$ equipped with $g_{0}$ is isometric to $\mathbb{S}^{3}$ (with the usual metric) with an open ball around one of the poles removed.

Note that $\left\{r=\frac{\pi}{2}\right\}$ corresponds to an equator of $\mathbb{S}^{3}$, and hence this is a surface ruled by stably trapped geodesics. It is the presence of this stable trapping that will 
eventually lead to the desired slow logarithmic decay rate for waves, in accordance also with the results on ultracompact neutron stars of [44]

We set $\mathcal{M} \doteq \mathbb{R} \times \mathbb{R}^{3}$, and we construct the Lorentzian metric $g$ on $\mathcal{M}$ so that in the coordinate system $(t, r, \vartheta, \omega),{ }^{37} g$ takes the form

$$
g=-d t^{2}+d r^{2}+f(r) \cdot\left(d \vartheta^{2}+\sin ^{2} \vartheta \cdot d \omega^{2}\right) .
$$

Hence, for any coordinate chart $\left(x^{1}, x^{2}, x^{3}\right)$ on $\mathbb{R}^{3}$ we have

$$
g=-d t^{2}+\left(g_{0}\right)_{\mu \nu} d x^{\mu} d x^{\nu}
$$

We readily verify that $(\mathcal{M}, g)$ satisfies assumptions $1,2,3$ and 4 :

- $(\mathcal{M}, g)$ is a stationary spacetime (with Killing vector field $T \doteq \partial_{t}$ ), with Cauchy hypersurface $\Sigma_{0} \doteq\{t=0\}$, which is asymptotically flat with one end (since outside $\{r \leq \pi\} g$ is the usual Minkowski metric, and the $\Sigma_{0}$ has Euclidean induced metric and vanishing second fundamental form). Hence, Assumption 1 is satisfied.

- There is no event horizon in $(\mathcal{M}, g)$, since any point of $\mathcal{M}$ communicates with the flat region $\{r \geq \pi\}$ with both future directed and past directed timelike curves: For any $p \in \mathcal{M}$ and any smooth curve $\delta:[0,1] \rightarrow \mathbb{R}^{3}$ such that $\delta(0)=\tilde{p}(\tilde{p}$ being the projection of $p$ on the second factor of $\mathcal{M}=\mathbb{R} \times \mathbb{R}^{3}$ ) and $\delta(1) \in\{r \geq \pi\}$, $^{38}$ we construct the smooth curve $\Gamma:[0,1] \rightarrow \mathcal{M}, \Gamma(\lambda) \doteq(t(p)+C \cdot \lambda, \delta(\lambda))$, for some $C \gg 1$. We see that $\Gamma(0)=p, \Gamma(1) \in\{r \geq \pi\}$ and, if $C \gg 1$ is sufficiently large, due to $12.2, \Gamma$ is timelike future oriented. Similarly, if we had instead chosen $C \ll-1, \Gamma$ would have been timelike past directed. Thus, assumption Assumption 2 is satisfied.

- There is no ergoregion, since $g(T, T) \equiv-1$, and thus assumption 3 holds trivially.

- The boundedness of the energy of solutions to the wave equation holds trivially, since $\mathrm{T}$ is globally timelike and $\int_{t=\tau} J_{\mu}^{T}(\psi) n^{\mu}$ is constant (and hence bounded) for any smooth solution $\psi$ to the wave equation $\square_{g} \psi=0$ on $(\mathcal{M}, g)$. So Assumption 4 is also true.

The fact that Theorem 2.1 is optimal for the spacetime $(\mathcal{M}, g)$ is a consequence of the following proposition:

Proposition 12.1 For any function $h:[0,+\infty) \rightarrow[0,+\infty)$ for which $h(t) \cdot\left\{\log ^{2}(2+\right.$ $t)\} \rightarrow 0$ as $t \rightarrow+\infty$, there exists a $t_{0}>0$ so that for any $\tau>t_{0}$, one can construct a smooth solution $\psi$ (depending on $\tau)$ to the wave equation on $(\mathcal{M}, g)$ with finite $T$-energy on the $\{t=$ const $\}$ hypersurfaces such that

$$
\int_{\{t=\tau\} \cap\left\{r \leq \frac{3 \pi}{4}\right\}} J_{\mu}^{T}(\psi) n^{\mu}>h(\tau) \cdot\left(\int_{t=0}(1+r) \cdot J_{\mu}^{T}(\psi) n^{\mu}+\int_{t=0} J_{\mu}^{T}(T \psi) n^{\mu}\right) .
$$

\footnotetext{
${ }^{37} t$ denoting the projection on the first factor of $\mathbb{R} \times \mathbb{R}^{3}$, while $(r, \vartheta, \omega)$ are as before the usual polar coordinates on $\mathbb{R}^{3}$.

${ }^{38}$ Such a curve always exist, it can be e.g. a straight line in $\mathbb{R}^{3}$.
} 
Proof This is accomplished through the quasimode construction performed by Rodnianski and Tao in [58] in the following way:

We first note that on $\mathbb{S}^{3}$, equipped with the usual coordinate system $\left(\vartheta_{1}, \vartheta_{2}, \vartheta_{3}\right)$ in which the metric takes the form $g_{\mathbb{S}^{3}}=d \vartheta_{1}^{2}+\sin ^{2} \vartheta_{1} \cdot\left(d \vartheta_{2}^{2}+\sin ^{2} \vartheta_{2} \cdot d \vartheta_{3}^{2}\right)$, the Laplacian takes the form

$$
\Delta_{\mathbb{S}^{3}}=\partial_{\vartheta_{1}}^{2}+2 c \cot \vartheta_{1} \cdot \partial_{\vartheta_{1}}+\frac{1}{\sin ^{2} \vartheta_{1}} \Delta_{\mathbb{S}^{2}}
$$

( $\Delta_{\mathbb{S}^{2}}$ being the Laplace-Beltrami of the usual metric of $\mathbb{S}^{2}$ in the coordinates $\left(\vartheta_{2}, \vartheta_{3}\right)$ ).

For any integer $l \geq 0$, we will denote with $Y_{l}=Y_{l}\left(\vartheta_{2}, \vartheta_{3}\right)$ a spherical harmonic on $\mathbb{S}^{2}$ of order $l$, that is a function satisfying $\Delta_{\mathbb{S}^{2}} Y_{l}+l^{2} Y_{l}=0$, normalised so that $\int_{\mathbb{S}^{2}}\left|Y_{l}\right|^{2}=1$. Then, the function $u_{l}: \mathbb{S}^{3} \rightarrow \mathbb{C}$ defined by $u_{l}\left(\vartheta_{1}, \vartheta_{2}, \vartheta_{3}\right)=\sin ^{l}\left(\vartheta_{1}\right)$. $\cos \left(\vartheta_{1}\right) \cdot Y_{l}\left(\vartheta_{2}, \vartheta_{3}\right)$ satisfies the equation

$$
\Delta_{\mathbb{S}^{3}} u_{l}+\stackrel{2}{\lambda} u_{l}=0
$$

where $\lambda^{2} \doteq(l+1) \cdot(l+3)$ (and hence $\lambda \sim l$ if $\left.l \gg 1\right)$

It is easy (see [58]) to obtain the following estimates for $u_{l}$, which are in fact quantitative expressions of the fact that the $u_{l}$ 's become more and more concentrated around $\vartheta_{1}=\frac{\pi}{2}$ as $l \rightarrow \infty$ (which can be also deduced in view of the $l$ exponent in $\sin ^{l}\left(\vartheta_{1}\right)$ in the definition of $\left.u_{l}\right)$ :

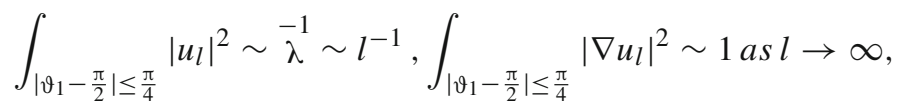

as well as

$$
\int_{\left|\vartheta_{1}-\frac{\pi}{2}\right|>\frac{\pi}{4}}\left(\left|u_{l}\right|^{2}+\left|\nabla u_{l}\right|^{2}\right)=O\left(e^{-c \lambda}\right)
$$

for some $c>0$.

We now fix a smooth cut-off function $\chi: \mathbb{S}^{3} \rightarrow[0,1]$ such that $\chi \equiv 1$ for $\vartheta_{1} \leq \frac{3 \pi}{4}$ and $\chi \equiv 0$ for $\vartheta_{1} \geq \frac{7 \pi}{8}$. We define

$$
\tilde{u}_{l} \doteq \chi \cdot u_{l}
$$

and we note that the $\tilde{u}_{l}$ 's are supported in $\left\{\vartheta_{1} \leq \frac{7 \pi}{8}\right\}$. Due to this fact, as well as the fact we can isometrically identify $\left\{r \leq \frac{7 \pi}{8}\right\} \subseteq \mathbb{R}^{3}$ (equipped with $g_{0}$ ) with $\left(\mathbb{S}^{3} \backslash\left\{\vartheta_{1}>\frac{7 \pi}{8}\right\}, g_{\mathbb{S}^{3}}\right)$ through relabelling our coordinate maps $r \rightarrow \vartheta_{1}, \vartheta \rightarrow \vartheta_{2}$, $\omega \rightarrow \vartheta_{3}$, we can consider $\tilde{u}_{l}$ as having been defined on $\mathbb{R}^{3}$ and satisfying:

$$
\Delta_{g_{0}} \tilde{u}_{l}+\lambda^{2} \tilde{u}_{l}=2 \partial^{i} \chi \cdot \partial_{i} u_{l}+\left(\Delta_{g_{0}} \chi\right) \cdot u_{l}
$$

where we raise indices using $g_{0}$. Note that the right hand side of (12.9) is supported in $\left\{\frac{3 \pi}{4} \leq r \leq \frac{7 \pi}{8}\right\}$. 
Finally, we define $\tilde{\psi}_{l}: \mathcal{M} \rightarrow \mathbb{C}$,

$$
\tilde{\psi}_{l}(t, x) \doteq e^{i \lambda t} \cdot \tilde{u}_{l}(x)
$$

$\left(t \in \mathbb{R}, x \in \mathbb{R}^{3}\right)$. Note that $\tilde{\psi}_{l}$ is supported in $\left\{r \leq \frac{7 \pi}{8}\right\}$, and, due to (12.9) and (12.2), it satisfies the equation

$$
\square \tilde{\psi}_{l}=F_{l},
$$

where

$$
F_{l}(t, x)=e^{i \lambda t}\left\{2 \partial^{i} \chi(x) \cdot \partial_{i} u_{l}(x)+\left(\Delta_{g_{0}} \chi(x)\right) \cdot u_{l}(x)\right\}
$$

is supported in $\left\{\frac{3 \pi}{4} \leq r \leq \frac{7 \pi}{8}\right\}$.

Due to (12.6) and (12.7), for any $\tau \geq 0$ we can estimate (as $l \rightarrow \infty$ ):

$$
\begin{aligned}
& \int_{t=0} J_{\mu}^{T}\left(\tilde{\psi}_{l}\right) n^{\mu} \sim 1 \\
& \int_{t=0} J_{\mu}^{T}\left(T \tilde{\Psi}_{l}\right) n^{\mu} \sim l^{2}, \\
& \int_{\{t=\tau\} \cap\left\{r \leq \frac{3 \pi}{4}\right\}} J_{\mu}^{T}\left(\tilde{\Psi}_{l}\right) n^{\mu}=\int_{\{t=0\} \cap\left\{r \leq \frac{3 \pi}{4}\right\}} J_{\mu}^{T}\left(\tilde{\Psi}_{l}\right) n^{\mu} \sim 1
\end{aligned}
$$

and

$$
\int_{0}^{\tau}\left(\int_{\{t=s\}}\left|F_{l}\right|^{2}\right)^{1 / 2} d s=\tau \cdot O\left(e^{-c l}\right) .
$$

If we denote with $\psi_{l}$ the unique solution to the wave equation $\square \psi_{l}=0$ on $(\mathcal{M}, g)$, with initial data on $\{t=0\}$ being the induced initial data there by $\tilde{\psi}_{l}$, then we have

$$
\int_{t=0} J_{\mu}^{T}\left(\psi_{l}\right) n^{\mu}=\int_{t=0} J_{\mu}^{T}\left(\tilde{\psi}_{l}\right) n^{\mu} \sim 1
$$

Moreover, since $\square_{g}=-\partial_{t}^{2}+\Delta_{g_{0}}$, we calculate

$$
\left.T^{2} \psi_{l}\right|_{t=0}=\Delta_{g_{0}} \psi_{l}||_{t=0}=\left.\Delta_{g_{0}} \tilde{\psi}_{l}\right|_{t=0}=\left.T^{2} \tilde{\psi}_{l}\right|_{t=0}+\left.\square_{g} \tilde{\psi}_{l}\right|_{t=0}=\left.T^{2} \tilde{\psi}_{l}\right|_{t=0}+\left.F_{l}\right|_{t=0}
$$

Due to the fact that for any spatial derivative $\partial_{x^{i}}$ we have $\left.\partial_{x^{i}} \partial_{t} \psi_{l}\right|_{t=0}=\left.\partial_{x^{i}} \partial_{t} \tilde{\psi}_{l}\right|_{t=0},{ }^{39}$ we can estimate in view of (12.18):

$$
\left|\int_{t=0} J_{\mu}^{T}\left(T \psi_{l}\right) n^{\mu}\right| \leq \int_{t=0} J_{\mu}^{T}\left(T \tilde{\psi}_{l}\right) n^{\mu}+\int_{t=0}\left|F_{l}\right|^{2} \sim l^{2}+O\left(e^{-c l}\right) \sim l^{2} .
$$

\footnotetext{
39 Since $\partial_{t} \psi_{l}$ and $\partial_{t} \tilde{\psi}_{l}$ are identical on $\{t=0\}$.
} 
By an application of Duhamel's principle on $\psi_{l}-\tilde{\psi}_{l}$ (which satisfies $\square_{g}\left(\psi_{l}-\tilde{\psi}_{l}\right)=$ $-F_{l}$ and has vanishing initial data on $\{t=0\}$ ), we infer that for any $\tau \geq 0$ :

$$
\left(\int_{t=\tau} J_{\mu}^{T}\left(\psi_{l}-\tilde{\psi}_{l}\right) n^{\mu}\right)^{1 / 2} \leq C \cdot \int_{0}^{\tau}\left(\int_{\{t=s\}}\left|F_{l}\right|^{2}\right)^{1 / 2} d s \leq \tau \cdot O\left(e^{-c l}\right)
$$

and thus in view of (12.15) and (12.20):

$$
\begin{aligned}
\int_{\{t=\tau\} \cap\left\{r \leq \frac{3 \pi}{4}\right\}} J_{\mu}^{T}\left(\psi_{l}\right) n^{\mu} & \geq \int_{\{t=\tau\} \cap\left\{r \leq \frac{3 \pi}{4}\right\}} J_{\mu}^{T}\left(\tilde{\psi}_{l}\right) n^{\mu}-\int_{t=\tau} J_{\mu}^{T}\left(\psi_{l}-\tilde{\psi}_{l}\right) n^{\mu} \\
& \geq 1-C \tau \cdot e^{-c l}
\end{aligned}
$$

Finally, since $\left.\psi_{l}\right|_{t=0}$ and $\left.T \psi_{l}\right|_{t=0}$ are supported in $\left\{r \leq \frac{7 \pi}{8}\right\}$, we readily see that

$$
\int_{t=0}(1+r) J_{\mu}^{T}\left(\psi_{l}\right) n^{\mu} \sim \int_{t=0} J_{\mu}^{T}\left(\psi_{l}\right) n^{\mu} \sim 1
$$

From (12.17), (12.19), (12.21) and (12.22) we conclude that there exists a constant $b>0$ such that for all $l \gg 1$ and for any given $\tau>0$ :

$$
\begin{gathered}
\int_{\{t=\tau\} \cap\left\{r \leq \frac{3 \pi}{4}\right\}} J_{\mu}^{T}\left(\psi_{l}\right) n^{\mu}>b \cdot l^{-2}\left\{1-C \tau \cdot e^{-c l}\right\} \\
\cdot\left(\int_{t=0}(1+r) \cdot J_{\mu}^{T}\left(\psi_{l}\right) n^{\mu}+\int_{t=0} J_{\mu}^{T}\left(T \psi_{l}\right) n^{\mu}\right) .
\end{gathered}
$$

Thus, for any given function $h(t)=o\left(\log ^{-2}(t+2)\right)($ as $t \rightarrow \infty)$, we can find a large enough $t_{0} \gg 1$, such that for any given $\tau>t_{0}$, after picking $l \sim \frac{2}{c} \log (\tau+2)$ we can bound from (12.23):

$$
\begin{aligned}
& \int_{\{t=\tau\} \cap\left\{r \leq \frac{3 \pi}{4}\right\}} J_{\mu}^{T}\left(\psi_{l}\right) n^{\mu} . \\
& >b\left\{\frac{c^{2}}{4\{\log (\tau+2)\}^{2}}-C \tau \cdot \tau^{-2}\right\} \cdot\left(\int_{t=0}(1+r) \cdot J_{\mu}^{T}\left(\psi_{l}\right) n^{\mu}+\int_{t=0} J_{\mu}^{T}\left(T \psi_{l}\right) n^{\mu}\right) \\
& >h(t) \cdot\left(\int_{t=0}(1+r) \cdot J_{\mu}^{T}\left(\psi_{l}\right) n^{\mu}+\int_{t=0} J_{\mu}^{T}\left(T \psi_{l}\right) n^{\mu}\right) .
\end{aligned}
$$

Acknowledgments I would like to thank my advisor Mihalis Dafermos for suggesting this problem to me and providing me with comments, ideas and assistance while this paper was being written. I would also like to thank Igor Rodnianski for his invaluable ideas and suggestions. I would finally like to thank Yakov Shlapentokh-Rothman and Stefanos Aretakis for many insightful conversations and important comments on preliminary versions of this paper. 


\section{A Construction of the $(u, r, \sigma)$ Coordinate Chart}

Assume that an open subset $\mathcal{U}$ of a spacetime $(\mathcal{M}, g)$ admits a polar coordinate chart $(t, r, \sigma)$ for $\{r \geq R>0\}$, where, for some integer $m \geq 1$, the metric $g$ has the expression:

$$
\begin{aligned}
g= & -\left(1-\frac{2 M}{r}+O_{m}\left(r^{-1-a}\right)\right) d t^{2}+\left(1+\frac{2 M}{r}+O_{m}\left(r^{-1-a}\right)\right) d r^{2} \\
& +r^{2}\left(g_{\mathbb{S} d-1}+O_{m}\left(r^{-1-a}\right)\right)+O_{m}\left(r^{-a}\right) d t d \sigma
\end{aligned}
$$

where $M \in \mathbb{R}$ and no metric coefficient depends on the $t$ coordinate; this means that the vector field $T=\partial_{t}$ is Killing. In the above, the $O_{m}\left(r^{b}\right)$ notation is used to denote functions or tensors $h$ on $\mathbb{S}^{d-1}$ depending on $(r, \sigma)$ and satisfying the bound:

$$
\sum_{j=0}^{m}\left(\sum_{j_{1}+j_{2}=j} r^{j_{1}}\left\|\partial_{r}^{j_{1}} \partial_{\sigma}^{j_{2}} h(r, \sigma)\right\|_{g_{\mathbb{S} d-1}}\right) \leq C \cdot r^{b} .
$$

We will show that we can find a new coordinate chart $(u, r, \sigma)$ on $\mathcal{U}$ in which $g$ will take the form:

$$
\begin{aligned}
g= & -4\left(1-\frac{2 M}{r}+O_{m-1}\left(r^{-1-a}\right)\right) d u^{2}-4\left(1+O_{m-1}\left(r^{-1-a}\right)\right) d u d r \\
& +r^{2}\left(g_{\mathbb{S}^{d-1}}+O_{m-1}\left(r^{-1-a}\right)\right)+O_{m-1}\left(r^{-a}\right) d u d \sigma+O_{m-1}\left(r^{-a}\right) d r d \sigma .
\end{aligned}
$$

In order to construct the required coordinate function $u$, we first introduce an auxiliary coordinate function $r^{*}=r^{*}(r, \sigma)$, the analogue of the Regge-Wheeler coordinate function for the Schwarzschild spacetime. The main identity that we will need to hold is $g_{r^{*} r^{*}}=-g_{t t}$ in the $\left(t, r^{*}, \sigma\right)$ coordinate system. From the expression of the metric (12.25), we see that $\sqrt{-\frac{g_{r r}}{g_{t t}}}=\left(1-\frac{2 M}{r}\right)^{-1}+h(r, \sigma)$, for a smooth function $h(r, \sigma)=O_{m}\left(r^{-1-a}\right)$. Therefore, we have to define:

$$
r^{*}(r, \sigma) \doteq r+2 M \cdot \log (r-2 M)-\int_{r}^{+\infty} h(\rho, \sigma) d \rho .
$$

Due to the fact that $h=O_{m}\left(r^{-1-a}\right), r^{*}$ is a continuous function of $(r, \sigma)$ satisfying $\frac{\partial r^{*}}{\partial r}=\left(1-\frac{2 M}{r}\right)^{-1}+h(r, \sigma)$ as desired. Moreover

$$
\partial_{\sigma} r^{*}(r, \sigma)=-\int_{r}^{+\infty} \partial_{\sigma} h(\rho, \sigma) d \rho=O_{m-1}\left(r^{-a}\right) .
$$

Hence, in the coordinate system $\left(t, r^{*}, \sigma\right)$ (which is indeed a coordinate system for $r \gg 1$ due to the form of $r^{*}$ ) we can compute that the metric takes the following form: 


$$
\begin{aligned}
g= & \left(1-\frac{2 M}{r}+O_{m-1}\left(r^{-1-a}\right)\right)\left(-d t^{2}+\left(d r^{*}\right)^{2}\right)+r^{2}\left(g_{\mathbb{S} d-1}+O_{m-1}\left(r^{-1-a}\right)\right) \\
& +O_{m-1}\left(r^{-a}\right) d r^{*} d \sigma+O_{m-1}\left(r^{-a}\right) d t d \sigma .
\end{aligned}
$$

We can now introduce the function $u=\frac{1}{2}\left(t-r^{*}\right)$. In the $\left(u, r^{*}, \sigma\right)$ coordinates, the metric takes the form:

$$
\begin{aligned}
g= & -4\left(1-\frac{2 M}{r}+O_{m-1}\left(r^{-1-a}\right)\right)\left(d u^{2}+d u d r^{*}\right)+r^{2}\left(g_{\mathbb{S}^{d-1}}+O_{m-1}\left(r^{-1-a}\right)\right) \\
& +O_{m-1}\left(r^{-a}\right) d u d \sigma+O_{m-1}\left(r^{-a}\right) d r^{*} d \sigma .
\end{aligned}
$$

Thus, switching finally to the $(u, r, \sigma)$ coordinate system, since $\partial_{r} r^{*}=1+\frac{2 M}{r}+$ $O_{m-1}\left(r^{-1-a}\right)$, we deduce that the metric has the required expression:

$$
\begin{aligned}
g= & -4\left(1-\frac{2 M}{r}+O_{m-1}\left(r^{-1-a}\right)\right) d u^{2}-4\left(1+O_{m-1}\left(r^{-1-a}\right)\right) d u d r \\
& +r^{2}\left(g_{\mathbb{S}^{d-1}}+O_{m-1}\left(r^{-1-a}\right)\right)+O_{m-1}\left(r^{-a}\right) d u d \sigma+O_{m-1}\left(r^{-a}\right) d r d \sigma
\end{aligned}
$$

\section{B Proof of the Inclusion $J^{-}(p) \cap \Sigma \subseteq \Sigma \cap \mathcal{D}$ for $p \in J^{+}(\Sigma) \cap \mathcal{D}$}

Let $\left(\mathcal{M}^{d+1}, g\right), d \geq 3$ be a globally hyperbolic spacetime with a Cauchy hypersurface $\Sigma$. Let $\Sigma$ be asymptotically flat (in the sense of Assumption 1), and let $\mathcal{D}$ be the domain of outer communications associated to one asymptotically flat region $\mathcal{I}_{\text {as }}$ of $(\mathcal{M}, g)$ (see Assumption 1 for the relevant definitions). We also define

$$
\mathcal{H} \doteq \partial \mathcal{D}
$$

and we set

$$
\mathcal{H}^{+} \doteq J^{+}\left(\mathcal{I}_{\text {as }}\right) \cap \partial\left(J^{-}\left(\mathcal{I}_{\text {as }}\right)\right)
$$

and

$$
\mathcal{H}^{-} \doteq J^{-}\left(\mathcal{I}_{\text {as }}\right) \cap \partial\left(J^{+}\left(\mathcal{I}_{\text {as }}\right)\right)
$$

We will establish the following inclusion:

Lemma 12.2 With $\Sigma, \mathcal{D}, \mathcal{H}^{+}$and $\mathcal{H}^{-}$as above, under the additional assumption that $\mathcal{H}^{-} \subset I^{-}(\Sigma \cap \mathcal{D})$, the following inclusion holds:

$$
J^{-}(p) \cap \Sigma \subseteq \Sigma \cap \mathcal{D}
$$

for any $p \in J^{+}(\Sigma) \cap \mathcal{D}$. 
Proof The proof will follow by contradiction: if

$$
J^{-}(p) \cap \Sigma \subsetneq \Sigma \cap \mathcal{D}
$$

then there exists a past directed causal curve $\delta:[0,1) \rightarrow \mathcal{M}$ which is past inextendible with $\delta(0)=p$, and for which $\delta \cap(\Sigma \cap \mathcal{D})=\emptyset$. But then,

$$
\delta \subseteq J^{-}\left(\mathcal{I}_{a s}\right)
$$

since $p \in \mathcal{D} \subseteq J^{-}\left(\mathcal{I}_{a s}\right)$. Furthermore, $\delta \cap \Sigma \neq \emptyset$, since $\Sigma$ is a Cauchy hypersurface of $(\mathcal{M}, g)$. Thus, we have

$$
\delta \cap\left(\Sigma \cap J^{-}\left(\mathcal{I}_{a s}\right)\right) \neq \emptyset .
$$

If, in addition to (12.34), $\delta$ is entirely contained also in $J^{+}\left(\mathcal{I}_{a s}\right)$, then we would deduce from (12.35) that $\left(\delta \cap J^{+}\left(\mathcal{I}_{a s}\right)\right) \cap\left(\Sigma \cap J^{-}\left(\mathcal{I}_{a s}\right)\right) \neq \varnothing$, or equivalently (since $\left.\mathcal{D}=J^{+}\left(\mathcal{I}_{a s}\right) \cap J^{-}\left(\mathcal{I}_{a s}\right)\right)$ that $\delta \cap(\mathcal{D} \cap \Sigma) \neq \emptyset$, which is the required contradiction.

If, on the other hand, $\delta$ is not entirely contained in $J^{+}\left(\mathcal{I}_{a s}\right)$, then $\delta \cap \partial J^{+}\left(\mathcal{I}_{a s}\right)$ should be non empty, which implies that

$$
\delta \cap \mathcal{H}^{-} \neq \varnothing
$$

because of (12.34) and the fact that $\mathcal{H}^{-}=J^{-}\left(\mathcal{I}_{\text {as }}\right) \cap \partial J^{+}\left(\mathcal{I}_{\text {as }}\right)$. Therefore, let

$$
\underset{0}{\lambda}=\inf \left\{\lambda \in[0,1): \delta(\lambda) \in \mathcal{H}^{-}\right\}
$$

Since $\mathcal{H}^{-}$is closed by definition, $\delta\left(\lambda_{0}\right) \in \mathcal{H}^{-}$, and due to the definition of $\lambda_{0}$, we have

$$
\delta([0, \underset{0}{\lambda}]) \subset J^{+}\left(\mathcal{I}_{a s}\right) \cap J^{-}\left(\mathcal{I}_{a s}\right)=\mathcal{D} .
$$

In view of our assumption that $\mathcal{H}^{-} \subset J^{-}(\Sigma \cap \mathcal{D}) \subseteq J^{-}\left(\Sigma \cap J^{-}\left(\mathcal{I}_{\text {as }}\right)\right)$ and the facts that $\delta\left(\lambda_{0}\right) \in \mathcal{H}^{-}$and $\delta$ is causal and past directed,

$$
\delta(\underset{0}{(\lambda, 1)}) \cap\left(\Sigma \cap J^{-}\left(\mathcal{I}_{a s}\right)\right)=\emptyset .
$$

can not intersect $\Sigma \cap J^{-}\left(\mathcal{I}_{a s}\right)$. However, in view of (12.35), there exists some $\lambda_{1} \in$ $[0,1)$ such that $\delta\left(\lambda_{1}\right) \in \Sigma \cap J^{-}\left(\mathcal{I}_{a s}\right)$. Thus, from (12.39) we infer that $\lambda_{1} \in\left[0, \lambda_{0}\right]$. But since $\delta\left(\left[0, \lambda_{0}\right]\right) \subset \mathcal{D}$, we obtain that $\delta \cap(\Sigma \cap \mathcal{D}) \neq \emptyset$, which again is the required contradiction.

Hence, we conclude that for any $p \in J^{+}(\Sigma) \cap \mathcal{D}$ we have $J^{-}(p) \cap \Sigma \subseteq \Sigma \cap \mathcal{D}$.

\section{Interpolation for $\boldsymbol{r}$-Weighted Energy Bounds}

Lemma 12.3 With the notations as in Section 8, if for any smooth solution $\psi$ to $\square_{g} \psi=0$ on $J^{+}(\Sigma) \cap \mathcal{D}$ with compactly supported initial data on $\Sigma \cap \mathcal{D}$ and for any $\tau \in\left[\frac{1}{4} t^{*}, \frac{3}{4} t^{*}\right]$ we can bound 


$$
\int_{S_{\tau} \cap \mathcal{R}\left(0, t^{*}\right)} J_{\mu}^{N}\left(\psi_{\leq \omega_{+}}\right) n_{S_{\tau}}^{\mu} \leq \frac{C}{\tau-\frac{1}{4} t^{*}} \cdot\left\{e^{C \omega_{+}} \int_{t=0} J_{\mu}^{N}(\psi) n^{\mu}+\int_{t=0} r J_{\mu}^{N}(\psi) n^{\mu}\right\}
$$

and

$$
\int_{S_{\tau} \cap \mathcal{R}\left(0, t^{*}\right)} J_{\mu}^{N}\left(\psi_{\leq \omega_{+}}\right) n_{S_{\tau}}^{\mu} \leq C \int_{t=0} J_{\mu}^{N}(\psi) n^{\mu},
$$

then for any $\delta_{0} \in[0,1]$ and any $\tau \in\left[\frac{1}{4} t^{*}, \frac{3}{4} t^{*}\right]$ we can also bound

$$
\int_{S_{\tau} \cap \mathcal{R}\left(0, t^{*}\right)} J_{\mu}^{N}\left(\psi_{\leq \omega_{+}}\right) n_{S_{\tau}}^{\mu} \leq \frac{C}{\left(\tau-\frac{1}{4} t^{*}\right)^{\delta 0}} \cdot\left\{e^{C \omega_{+}} \int_{t=0} J_{\mu}^{N}(\psi) n^{\mu}+\int_{t=0} r^{\delta 0} J_{\mu}^{N}(\psi) n^{\mu}\right\} .
$$

Proof For any $\tau \in\left[\frac{1}{4} t^{*}, \frac{3}{4} t^{*}\right]$, we will define the following energy norm on the smooth and compactly supported pairs of functions $\left(\omega_{0}, \omega_{1}\right)$ on the hypersurface $S_{\tau} \cap \mathcal{R}\left(0, t^{*}\right)$ :

$$
\left\|\left(\omega_{0}, \omega_{1}\right)\right\|_{\mathcal{H}_{e n, \tau}^{1}} \doteq \int_{S_{\tau} \cap \mathcal{R}\left(0, t^{*}\right)} J_{\mu}^{N}(\omega) n_{S_{\tau}}^{\mu}
$$

where $\omega$ is the unique solution on $J^{+}\left(S_{\tau}\right) \cap \mathcal{R}\left(0, t^{*}\right)$ with $\left.\omega\right|_{S_{\tau} \cap \mathcal{R}\left(0, t^{*}\right)}=\omega_{0}$ and $\left.N \omega\right|_{S_{\tau} \cap \mathcal{R}\left(0, t^{*}\right)}=\omega_{1}$. We will also denote with $\|\cdot\|_{\mathcal{H}_{e n, \tau}^{-1}}$ the dual norm of $\|\cdot\|_{\mathcal{H}_{e n, \tau}^{1}}$ on the space of smooth and compactly supported pairs on $S_{\tau} \cap \mathcal{R}\left(0, t^{*}\right)$, that is to say:

$$
\left\|\left(\omega_{0}, \omega_{1}\right)\right\|_{\mathcal{H}_{e n, \tau}^{-1}}=\sup _{\left\{\left(w_{0}, w_{1}\right):\left\|\left(w_{0}, w_{1}\right)\right\|_{\mathcal{H}_{e n, \tau}^{1}}=1\right\}} \int_{S_{\tau} \cap \mathcal{R}\left(0, t^{*}\right)} \operatorname{Re}\left\{\omega_{0} \bar{w}_{0}+\omega_{1} \bar{w}_{1}\right\} .
$$

For any complex number $s$ in the strip $\{0 \leq \operatorname{Re}(s) \leq 1\}$, we define the function $\psi^{(s)}: \mathcal{D}\left(\Sigma_{0}\right) \rightarrow \mathbb{C}$ (where $\mathcal{D}\left(\Sigma_{0}\right)$ is the domain of dependence of $\Sigma_{0}$ ) as the unique solution of the following initial value problem

$$
\left\{\begin{array}{l}
\square \psi^{(s)}=0 \\
\psi^{(s)}\left|\Sigma_{0}=(1+r)^{\frac{s}{2}\left(\delta_{0}-1\right)+\frac{(1-s)}{2} \delta_{0}} \psi\right|_{\Sigma_{0}}, \\
\left.N \psi^{(s)}\right|_{\Sigma_{0}}=N\left((1+r)^{\frac{s}{2}\left(\delta_{0}-1\right)+\frac{(1-s)}{2} \delta_{0}} \psi\right) \mid \Sigma_{0}
\end{array}\right.
$$

Notice that $\psi^{\left(\delta_{0}\right)} \equiv \psi$. For any pair $w=\left(w_{0}, w_{1}\right)$ of smooth and compactly supported functions on $S_{\tau} \cap \mathcal{R}\left(0, t^{*}\right)$ with $\|w\|_{\mathcal{H}_{e n, \tau}^{-1}}=1$, we also introduce the following function

$$
F_{w}[\psi](s) \doteq \int_{S_{\tau}} \operatorname{Re}\left\{\left(\tau-\frac{1}{4} t^{*}\right)^{\frac{s}{2}} \psi_{\leq \omega_{+}}^{(s)} \cdot \bar{w}_{0}+\left(\tau-\frac{1}{4} t^{*}\right)^{\frac{s}{2}} N\left(\psi_{\leq \omega_{+}}^{(s)}\right) \cdot \bar{w}_{1}\right\}
$$

which is holomorhic in $s$ in the strip $\{0 \leq \operatorname{Re}(s) \leq 1\}$. When $s=0+\rho i$ with $\rho \in \mathbb{R}$, an application of (12.41) for $\psi^{(\rho i)}$ in place of $\psi$ readily yields (after also applying a Cauchy-Schwarz inequality on (12.46), in view of the fact that $\|w\|_{\mathcal{H}_{e n, \tau}^{-1}}=1$ ) that 


$$
\begin{aligned}
\left|F_{w}[\psi](i \rho)\right| & \leq C \int_{t=0}\left((1+r)^{\delta_{0}} J_{\mu}^{N}(\psi) n^{\mu}+(1+r)^{\delta_{0}-2}|\psi|^{2}\right) \\
& \leq C \int_{t=0}(1+r)^{\delta_{0}} J_{\mu}^{N}(\psi) n^{\mu}
\end{aligned}
$$

(the second line following from the first after an application of a Hardy-type inequality). When $s=1+\rho i$ with $\rho \in \mathbb{R}$, an application of (12.40) for $\psi^{(1+\rho i)}$ in place of $\psi$ yields (after applying again a Cauchy-Schwarz inequality on (12.46), in view of the fact that $\left.\|w\|_{\mathcal{H}_{e n, \tau}^{-1}}=1\right)$, that

$$
\begin{aligned}
\left|F_{w}[\psi](1+i \rho)\right| & \leq C\left\{e ^ { C \omega _ { + } } \int _ { t = 0 } \left((1+r)^{\delta 0-1} J_{\mu}^{N}(\psi) n^{\mu}\right.\right. \\
& \left.\left.+(1+r)^{\delta 0-3}|\psi|^{2}\right)+\int_{t=0}\left((1+r)^{\delta 0} J_{\mu}^{N}(\psi) n^{\mu}+(1+r)^{\delta 0-2}|\psi|^{2}\right)\right\} \\
& \leq C\left\{e^{C \omega_{+}} \int_{t=0} J_{\mu}^{N}(\psi) n^{\mu}+\int_{t=0} r^{\delta_{0}} J_{\mu}^{N}(\psi) n^{\mu}\right\} .
\end{aligned}
$$

By applying the Phragmen-Lindelöf maximum principle on the function $F_{w}[\psi](s)$ on the strip $\{0 \leq \operatorname{Re}(s) \leq 1\}$ and using (12.47) and (12.48) we obtain

$$
\left|F_{w}[\psi]\left(\delta_{0}\right)\right| \leq C\left\{e^{C \omega_{+}} \int_{t=0} J_{\mu}^{N}(\psi) n^{\mu}+\int_{t=0} r^{\delta_{0}} J_{\mu}^{N}(\psi) n^{\mu}\right\},
$$

that is to say

$$
\begin{aligned}
& \left|\int_{S_{\tau}} \operatorname{Re}\left\{\tau^{\frac{\delta_{0}}{2}} \psi_{\leq \omega_{+}} \cdot \bar{w}_{0}+\tau^{\frac{\delta_{0}}{2}} N\left(\psi_{\leq \omega_{+}}\right) \cdot \bar{w}_{1}\right\}\right| \\
& \quad \leq C\left\{e^{C \omega_{+}} \int_{t=0} J_{\mu}^{N}(\psi) n^{\mu}+\int_{t=0} r^{\delta 0} J_{\mu}^{N}(\psi) n^{\mu}\right\} .
\end{aligned}
$$

Since (12.50) holds for all pairs $w=\left(w_{0}, w_{1}\right)$ with $\|w\|_{\mathcal{H}_{e n, \tau}^{-1}}=1$, from (12.50) and the definition (12.43) of the $\mathcal{H}_{e n, \tau}^{1}$ norm, we finally obtain (12.42).

\section{References}

1. Andersson, L., Blue, P.: Hidden symmetries and decay for the wave equation on the Kerr spacetime. Ann. Math. 182(3), 787-853 (2015)

2. Aretakis, S.: Stability and instability of extreme Reissner-Nordström black hole spacetimes for linear scalar perturbations II. Annales Henri Poincaré 12(8), 1491-1538 (2011)

3. Aretakis, S.: Stability and instability of extreme Reissner-Nordström black hole spacetimes for linear scalar perturbations I. Commun. Math. Phys. 307(1), 17-63 (2011)

4. Aretakis, S.: Decay of axisymmetric solutions of the wave equation on extreme Kerr backgrounds. J. Funct. Anal. 263(9), 2770-2831 (2012)

5. Aretakis, S.: Horizon instability of extremal black holes. arXiv:1206.6598, (2012)

6. Blue, P., Soffer, A.: Semilinear wave equations on the Schwarzschild manifold I: Local decay estimates. arXiv:gr-qc/0310091, (2003) 
7. Blue, P., Sterbenz, J.: Uniform decay of local energy and the semi-linear wave equation on Schwarzschild space. Commun. Math. Phys. 268(2), 481-504 (2006)

8. Burq, N.: Décroissance de l'énergie locale de l'équation des ondes pour le problème extérieur et absence de résonance au voisinage du réel. Acta Math. 180(1), 1-29 (1998)

9. Cardoso, F., Vodev, G.: Uniform estimates of the resolvent of the Laplace-Beltrami operator on infinite volume Riemannian manifolds. II. Annales Henri Poincaré 3(4), 673-691 (2002)

10. Cardoso, F., Vodev, G.: High frequency resolvent estimates and energy decay of solutions to the wave equation. Can. Math. Bull. 47(4), 504-514 (2004)

11. Christodoulou, D.: Global solutions of nonlinear hyperbolic equations for small initial data. Commun. Pure Appl. Math. 39(2), 267-282 (1986)

12. Chrusciel, P., Wald, R.M.: On the topology of stationary black holes. Class. Quantum Gravity 11(12), L147 (1994)

13. Civin, D.: Stability of charged rotating black holes for linear scalar perturbations. $\mathrm{PhD}$ thesis, University of Cambridge, https://www.repository.cam.ac.uk/handle/1810/247397 (2014)

14. Dafermos, M., Rodnianski, I.: A proof of Price's law for the collapse of a self-gravitating scalar field. Invent. Math. 162(2), 381-457 (2005)

15. Dafermos, M., Rodnianski, I.: A note on energy currents and decay for the wave equation on a Schwarzschild background. arXiv preprint arXiv:0710.0171, (2007)

16. Dafermos, M., Rodnianski, I.: The wave equation on Schwarzschild-de Sitter spacetimes. arXiv preprint arXiv:0709.2766, (2007)

17. Dafermos, M., Rodnianski, I.: The black hole stability problem for linear scalar perturbations. In: Exner, P. (ed.) XVIth International Congress on Mathematical Physics, World Scientific, London, pp. 421-433, (2009)

18. Dafermos, M., Rodnianski, I.: The red-shift effect and radiation decay on black hole spacetimes. Commun. Pure Appl. Math. 62(7), 859-919 (2009)

19. Dafermos, M., Rodnianski, I.: Decay for solutions of the wave equation on Kerr exterior spacetimes I-II: the cases $|\mathrm{a}| \ll \mathrm{M}$ or axisymmetry. arXiv preprint arXiv:1010.5132 (2010)

20. Dafermos, M., Rodnianski, I.: A new physical-space approach to decay for the wave equation with applications to black hole spacetimes. In: XVIth International Congress on Mathematical, Physics, pp. 421-432. (2010)

21. Dafermos, M., Rodnianski, I.: A proof of the uniform boundedness of solutions to the wave equation on slowly rotating Kerr backgrounds. Invent. Math. 185(3), 467-559 (2011)

22. Dafermos, M., Rodnianski, I.: Lectures on black holes and linear waves. Evol. Equ. Clay Math. Proc. 17, 97-205 (2013)

23. Dafermos, M., Rodnianski, I., Shlapentokh-Rothman, Y.: Decay for solutions of the wave equation on Kerr exterior spacetimes III: the full subextremal case $|a|<M$. arXiv preprint arXiv:1402.7034, (2014)

24. Dafermos, M., Rodnianski, I., Shlapentokh-Rothman, Y.: A scattering theory for the wave equation on Kerr black hole exteriors. arXiv preprint arXiv:1412.8379, (2014)

25. Datchev, K.: Quantitative limiting absorption principle in the semiclassical limit. Geom. Funct. Anal. 24(3), 740-747 (2014)

26. Dimock, J.: Scattering for the wave equation on the schwarzschild metric. Gen. Relativ. Gravit. 17(4), 353-369 (1985)

27. Dimock, J., Kay, B.S.: Classical and quantum scattering theory for linear scalar fields on the Schwarzschild metric. II. J. Math. Phys. 27(10), 2520-2525 (1986)

28. Dimock, J., Kay, B.S.: Classical and quantum scattering theory for linear scalar fields on the Schwarzschild metric I. Ann. Phys. 175(2), 366-426 (1987)

29. Dold, D.: Unstable mode solutions to the Klein-Gordon equation in Kerr-anti-de Sitter spacetimes. arXiv preprint arXiv:1509.04971, (2015)

30. Dyatlov, S.: Exponential energy decay for Kerr-de Sitter black holes beyond event horizons. Math. Res. Lett. 18, 1023-1035 (2011)

31. Dyatlov, S.: Quasi-normal modes and exponential energy decay for the Kerr-de Sitter black hole. Commun. Math. Phys. 306(1), 119-163 (2011)

32. Elvang, H., Figueras, P.: Black saturn. J. High Energy Phys. 2007(05), 050 (2007)

33. Emparan, R., Reall, H.S.: Generalized Weyl solutions. Phys. Rev. D 65(8), 084025 (2002)

34. Emparan, R., Reall, H.S.: A rotating black ring solution in five dimensions. Phys. Rev. Lett. 88(10), 101101 (2002) 
35. Friedman, J.L.: Ergosphere instability. Commun. Math. Phys. 63(3), 243-255 (1978)

36. Friedman, J.L., Schleich, K., Witt, D.M.: Topological censorship. Phys. Rev. Lett. 71(10), 1486 (1993)

37. Gannot, O.: Quasinormal modes for Schwarzschild-AdS black holes: exponential convergence to the real axis. Commun. Math. Phys. 7, 1-29 (2014)

38. Holzegel, G.: Well-posedness for the massive wave equation on asymptotically anti-de Sitter spacetimes. J. Hyperbolic Differ. Equ. 9(02), 239-261 (2012)

39. Holzegel, G., Smulevici, J.: Decay properties of Klein-Gordon fields on Kerr-AdS spacetimes. Commun. Pure Appl. Math. 66(11), 1751-1802 (2013)

40. Holzegel, G., Smulevici, J.: Quasimodes and a lower bound on the uniform energy decay rate for Kerr-AdS spacetimes. arXiv preprint arXiv:1303.5944 (2013)

41. Ikawa, M.: Decay of solutions of the wave equation in the exterior of several convex bodies. Annales de l'institut Fourier 38(2), 113-146 (1988)

42. Jacobson, T., Venkataramani, S.: Topology of event horizons and topological censorship. Class. Quantum Gravity 12(4), 1055 (1995)

43. Kay, B.S., Wald, R.M.: Linear stability of Schwarzschild under perturbations which are non-vanishing on the bifurcation 2-sphere. Class. Quantum Gravity 4(4), 893 (1987)

44. Keir, J.: Slowly decaying waves on spherically symmetric spacetimes and an instability of ultracompact neutron stars. arXiv preprint arXiv:1404.7036 (2014)

45. Luk, J.: Improved decay for solutions to the linear wave equation on a Schwarzschild black hole. Annales Henri Poincaré 11(5), 805-880 (2010)

46. Luk, J.: A vector field method approach to improved decay for solutions to the wave equation on a slowly rotating Kerr black hole. Anal. PDE 5(3), 553-625 (2012)

47. Masarik, M.: The wave equation in a general spherically symmetric black hole geometry. Adv. Theor. Math. Phys. 15(6), 1789-1815 (2011)

48. Melrose, R., Sá Barreto, A., Vasy, A.: Asymptotics of solutions of the wave equation on de SitterSchwarzschild space. arXiv preprint arXiv:0811.2229 (2008)

49. Morawetz, C.S., Ralston, J.V., Strauss, W.A.: Decay of solutions of the wave equation outside nontrapping obstacles. Commun. Pure Appl. Math. 30(4), 447-508 (1977)

50. Moschidis, G.: A rigorous proof of Friedman's ergosphere instability for scalar waves. in preparation

51. Moschidis, G.: Superradiant instabilities arising for short-range non-negative potentials on Kerr spacetimes and applications. in preparation

52. Moschidis, G.: The $r^{p}$-weighted energy method of Dafermos and Rodnianski in general asymptotically flat spacetimes and applications. preprint

53. Nicolas, J.P.: Scattering of linear Dirac fields by a spherically symmetric black hole. Annales de l'IHP Physique théorique 62(2), 145-179 (1995)

54. Pomeransky, A., Sen'kov, R.: Black ring with two angular momenta. arXiv preprint hep-th/0612005 (2006)

55. Ralston, J.V.: Solutions of the wave equation with localized energy. Commun. Pure. Appl. Math. 22(6), 807-823 (1969)

56. Ralston, J.V.: Trapped rays in spherically symmetric media and poles of the scattering matrix. Commun. Pure Appl. Math. 24(4), 571-582 (1971)

57. Ringström, H.: The Cauchy Problem in General Relativity. European Mathematical Society, Zurich (2009)

58. Rodnianski, I., Tao, T.: Effective limiting absorption principles, and applications. arXiv preprint arXiv:1105.0873 (2011)

59. Sbierski, J.: Characterisation of the energy of Gaussian beams on Lorentzian manifolds-with applications to black hole spacetimes. arXiv preprint arXiv:1311.2477 (2013)

60. Schlag, W., Soffer, A., Staubach, W.: Decay for the wave and Schrödinger evolutions on manifolds with conical ends, Part I. Trans. Am. Math. Soc. 362(1), 19-52 (2010)

61. Schlag, W., Soffer, A., Staubach, W.: Decay for the wave and Schrödinger evolutions on manifolds with conical ends, Part II. Trans. Am. Math. Soc. 362(1), 289-318 (2010)

62. Schlue, V.: Decay of linear waves on higher-dimensional Schwarzschild black holes. Anal. PDE 6(3), 515-600 (2013)

63. Shlapentokh-Rothman, Y.: Exponentially growing finite energy solutions for the Klein-Gordon equation on sub-extremal Kerr spacetimes. Commun. Math. Phys. 329(3), 859-891 (2013)

64. Shlapentokh-Rothman, Y.: Quantitative mode stability for the wave equation on the Kerr spacetime. Annales Henri Poincaré 16, 1-57 (2013) 
65. Smoller, J.A., Wasserman, A.G., Yau, S.T.: Existence of black hole solutions for the EinsteinYang/Mills equations. Commun. Math. Phys. 154(2), 377-401 (1993)

66. Tataru, D., Tohaneanu, M.: A local energy estimate on Kerr black hole backgrounds. Int. Math. Res. Not. 2011(2), 248-292 (2011)

67. Vasy, A.: Microlocal analysis of asymptotically hyperbolic and Kerr-de Sitter spaces (with an appendix by Semyon Dyatlov). Invent. Math. 194(2), 381-513 (2013) 